\title{
Systems Biology Markup Language (SBML) Level 2: Structures and Facilities for Model Definitions
}

Michael Hucka

Andrew M. Finney

Stefan Hoops

Sarah M. Keating

Nicolas Le Novère
California Institute of Technology, USA

Oxford Computer Consultants Ltd, UK

Virginia Bioinformatics Institute, USA

University of Hertfordshire, $U K$

EMBL European Bioinformatics Institute, UK

sbml-editors@sbml.org

SBML Level 2 Version 3

Release 1

16 June 2007

Corrections and other changes to this SBML language specification may appear over time.

Notifications of new releases are broadcast on the mailing list sbml-announce@sbml.org

The latest release of the SBML Level 2 Version 3 specification is available at http://sbml.org/specifications/sbml-level-2/version-3/

This release of the specification is available at

http://sbml .org/specifications/sbml-level-2/version-3/release-1/

The list of known issues in all releases of SBML Level 2 Version 3 is available at http://sbml.org/specifications/sbml-level-2/version-3/errata/

The XML Schema for SBML Level 2 Version 3 is available at http://sbml.org/xml-schemas/ 


\section{Contents}

1 Introduction

1.1 Developments, discussions, and notifications of updates . . . . . . . . . . . . . . . . . . . . . 3

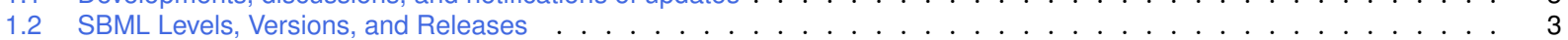

1.3 Language features and backward compatibility . . . . . . . . . . . . . . . . . . . . . . . . . 4

1.4 Document conventions . . . . . . . . . . . . . . . . . . . . . . . . . . . . . . . . . . .

2 Overview of SBML

3 Preliminary definitions and principles 11

3.1 Primitive data types . . . . . . . . . . . . . . . . . . . . . . . . . . . . . 11

3.2 Type SBase . . . . . . . . . . . . . . . . . . . . . . . . . . . . . . 13

3.3 The $\mathrm{id}$ and name attributes on SBML components . . . . . . . . . . . . . . . . . . . . . . . . . 18

3.4 Mathematical formulas in SBML Level 2 . . . . . . . . . . . . . . . . . . . . . . . . . . . . 20

4 SBML components 30

4.1 The SBML container . . . . . . . . . . . . . . . . . . . . . . . . . . . . . . . 30

4.2 Model . . . . . . . . . . . . . . . . . . . . . . . . . . . . . . . . . . 31

4.3 Function definitions . . . . . . . . . . . . . . . . . . . . . . . . . . . . . . . 33

4.4 Unit definitions . . . . . . . . . . . . . . . . . . . . . . . . . . . . . . . . . 35

4.5 Compartment types . . . . . . . . . . . . . . . . . . . . . . . . . . . . . . . . . . 42

4.6 Speciestypes . . . . . . . . . . . . . . . . . . . . . . . . . . . . . . 43

4.7 Compartments . . . . . . . . . . . . . . . . . . . . . . . . . . . . . . . . . . 44

4.8 Species . . . . . . . . . . . . . . . . . . . . . . . . . . . . . . 44

4.9 Parameters . . . . . . . . . . . . . . . . . . . . . . . . . . . . . 51

4.10 Initial assignments . . . . . . . . . . . . . . . . . . . . . . . . . . . . . . 53

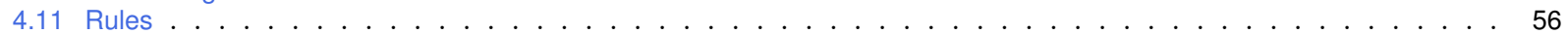

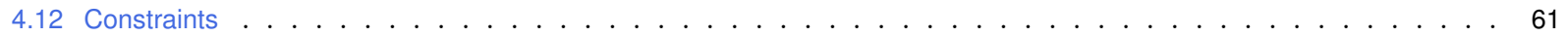

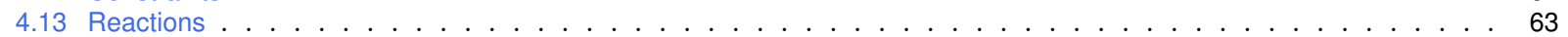

4.14 Events . . . . . . . . . . . . . . . . . . . . . . . . . . 77

5 The Systems Biology Ontology and the sboTerm attribute 83

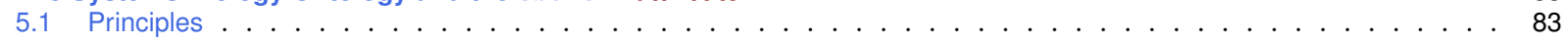

5.2 Using SBO and sboTerm . . . . . . . . . . . . . . . . . . . . . . . . . . . . . . . 84

5.3 Relationships to the SBML annotation element . . . . . . . . . . . . . . . . . . . . . . 89

5.4 Discussion . . . . . . . . . . . . . . . . . . . . . . . . . . . . . 89

6 A standard format for the annotation element 91

6.1 Motivation . . . . . . . . . . . . . . . . . . . . . . . . . . . . . . . . 91

6.2 XML namespaces in the standard annotation . . . . . . . . . . . . . . . . . . . . . . . . . . . . . . . . . . . . . . . . .

6.3 General syntax for the standard annotation . . . . . . . . . . . . . . . . . . . . . . . . . . . . . . . . . 92

6.4 Use of URls . . . . . . . . . . . . . . . . . . . . . . . . . . . . . . . . . . . . . . . . . . . . . . . . . . . . . . . . . . 993

6.5 Relation elements . . . . . . . . . . . . . . . . . . . . . . . . . . . . . . . . . . . . . . . . 94

6.6 Model history . . . . . . . . . . . . . . . . . . . . . . . . . . . . . . . . . . . . . . . . . . . . . . . . . . . . 994

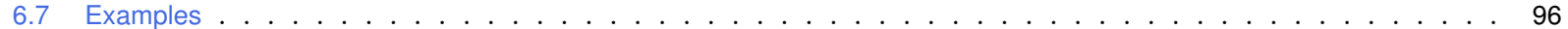

7 Example models expressed in XML using SBML 102

7.1 A simple example application of SBML . . . . . . . . . . . . . . . . . . . . . . . . . . . . . . . 102

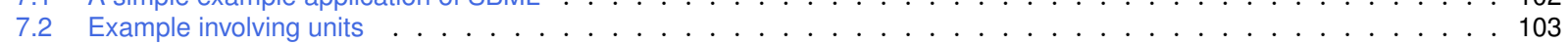

7.3 Example of a discrete version of a simple dimerization reaction . . . . . . . . . . . . . . . . . . . . . 105

7.4 Example involving assignment rules . . . . . . . . . . . . . . . . . . . . . . . . . 107

7.5 Example involving algebraic rules . . . . . . . . . . . . . . . . . . . . . . . . . . . . . . . . . . . . . 109

7.6 Example with combinations of boundaryCondition and constant values on Species with RateRule objects . . . . 111

7.7 Example of translation from a multi-compartmental model to ODEs . . . . . . . . . . . . . . . . . . . . . 112

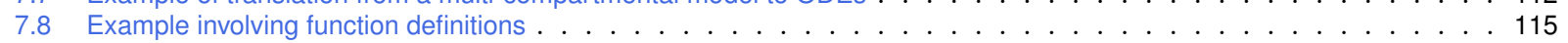

7.9 Example involving delay functions . . . . . . . . . . . . . . . . . . . . . . . . . . . . . . . . 116

7.10 Example involving events . . . . . . . . . . . . . . . . . . . . . . . . . . . . . . . 117

7.11 Example involving two-dimensional compartments . . . . . . . . . . . . . . . . . . . . . . . . . . . 119

8 Discussion 124

8.1 Future enhancements: SBML Level 3 and beyond . . . . . . . . . . . . . . . . . . . . . . . . . . . 124

Acknowledgments 126

A XML Schema for SBML $\quad 127$

B XML Schema for MathML subset 136

C Validation rules for SBML $\quad 140$

D A method for assessing whether an SBML model is overdetermined 153

E Mathematical consequences of the fast attribute on Reaction 156

F Processing and validating SBase notes and Constraint message content 158

G Major changes between versions of SBML Level 2 and implications for backward compatibility 159

G.1 Between Version 2 and Version 1 . . . . . . . . . . . . . . . . . . . . . . . . . . . . . . . . 159

G.2 Between Version 3 and Version $2 \ldots \ldots \ldots \ldots$

References 162 


\section{Introduction}

We present the Systems Biology Markup Language (SBML) Level 2 Version 3 Release 1, a model representation format for systems biology. SBML is oriented towards describing systems of biochemical reactions of the sort common in research on a number of topics, including cell signaling pathways, metabolic pathways, biochemical reactions, gene regulation, and many others. SBML is defined in a neutral fashion with respect to programming languages and software encoding; however, it is primarily oriented towards allowing models to be encoded using XML, the eXtensible Markup Language (Bosak and Bray, 1999; Bray et al., 2000). This document contains many examples of SBML models written in XML, as well as the text of an XML Schema (Biron and Malhotra, 2000; Fallside, 2000; Thompson et al., 2000) that defines SBML Level 2 Version 3. A downloadable copy of the XML Schema and other related documents and software are also available from the SBML project web site, http://sbml.org/.

The SBML project is not an attempt to define a universal language for representing quantitative models. The rapidly evolving views of biological function, coupled with the vigorous rates at which new computational techniques and individual tools are being developed today, are incompatible with a one-size-fits-all idea of a universal language. A more realistic alternative is to acknowledge the diversity of approaches and methods being explored by different software tool developers, and seek a common intermediate format - a lingua franca - enabling communication of the most essential aspects of the models.

The definition of the model description language presented here does not specify how programs should communicate or read/write SBML. We assume that for a simulation program to communicate a model encoded in SBML, the program will have to translate its internal data structures to and from SBML, use a suitable transmission medium and protocol, etc., but these issues are outside of the scope of this document.

\subsection{Developments, discussions, and notifications of updates}

SBML has been, and continues to be, developed in collaboration with an international community of researchers and software developers. As in many projects, the primary mode of interaction between members is electronic mail. Discussions about SBML take place on the mailing list sbml-discuss@caltech. edu. The mailing list archives and a web browser-based interface to the list are available at http://sbml .org/forums/.

A low-volume, broadcast-only mailing list is available where notifications of revisions to the SBML specification, notices of votes on SBML technical issues, and other critical matters are announced. This list is sbml-announce@caltech.edu and anyone may subscribe to it freely. This list will never be used for advertising and its membership list will never be disclosed. It is vitally important that all users of $S B M L$ stay informed about new releases and other developments by subscribing to this list, even if they do not wish to participate in discussions on the sbml-discuss@caltech.edu list. Please visit the SBML project web site, http://sbml.org/, for information about how to subscribe to sbml-announce@caltech.edu as well as for access to the list archives.

In Section 8.1, we attempt to acknowledge as many contributors to SBML's development as we can, but as SBML evolves, it becomes increasingly difficult to detail the individual contributions on a project that has truly become an international community effort.

\subsection{SBML Levels, Versions, and Releases}

Major editions of SBML are termed levels and represent substantial changes to the composition and structure of the language. The edition of SBML defined in this document, SBML Level 2, represents an evolution of the language resulting from the practical experiences of many users and developers working with SBML Level 1 since since its introduction in the year 2001 (Hucka et al., 2001, 2003). All of the constructs of Level 1 can be mapped to Level 2. In addition, a subset of Level 2 constructs can be mapped to Level 1. However, the levels remain distinct; a valid SBML Level 1 document is not a valid SBML Level 2 document, and likewise, a valid SBML Level 2 document is not a valid SBML Level 1 document.

Minor revisions of SBML are termed versions and constitute changes within a Level to correct, adjust, and refine language features. The present document defines SBML Level 2 Version 3. In Section 1.4.1 below 
explains how color is used in this document to indicate changes; a separate document provides a detailed listing of the changes between versions of SBML Level 2 as well as between SBML Level 2 Version 3 and SBML Level 1 Version 2.

Specification documents inevitably require minor editorial changes as its users discover errors and ambiguities. As a practical reality, these discoveries occur over time. In the context of SBML, such problems are formally announced publicly as errata in a given specification document. Borrowing concepts from the World Wide Web Consortium (Jacobs, 2004), we define SBML errata as changes of the following types: (a) formatting changes that do not result in changes to textual content; (b) corrections that do not affect conformance of software implementing support for a given combination of SBML Level and Version; and (c) corrections that may affect such software conformance, but add no new language features. A change that affects conformance is one that either turns conforming data, processors, or other conforming software into non-conforming software, or turns non-conforming software into conforming software, or clears up an ambiguity or insufficiently documented part of the specification in such a way that software whose conformance was once unclear now becomes clearly conforming or non-conforming (Jacobs, 2004). In short, errata do not change the fundamental semantics or syntax of SBML; they clarify and disambiguate the specification and correct errors. (New syntax and semantics are only introduced in SBML Versions and Levels.) An electronic tracking system for reporting and monitoring such issues is available at http://sbml.org/issue-tracker.

SBML errata result in new Releases of the SBML specification. Each release is numbered with an integer, with the first release of the specification being called release number 1. Subsequent releases of an SBML specification document contain a section listing the accumulated errata reported and corrected since the first release. A complete list of the errata for SBML Level 2 Version 3 since the publication of Release 1 is also made publicly available at http://sbml.org/specifications/sbml-level-2/version-3/errata/. Announcements of errata, releases of the SBML specification and other major changes are made on the sbml-announce@caltech.edu mailing list.

\subsection{Language features and backward compatibility}

Some language features of previous SBML Levels and Versions have been either deprecated or removed entirely in SBML Level 2 Version 3. For the purposes of SBML specifications, the following are the definitions of deprecated feature and removed feature:

Removed language feature: A syntactic construct that was present in previous SBML Levels and/or Versions within a Level, and has been removed beginning with a specific SBML Level and Version. Models containing such constructs do not conform to the specification of that SBML Level and Version.

Deprecated language feature: A syntactic construct that was present in previous SBML Levels and/or Versions within a Level, and while still present in the language definition, has been identified as nonessential and planned for future removal. Beginning with the Level and Version in which a given feature is deprecated, software tools should not generate SBML models containing the deprecated feature; however, for backward compatibility, software tools reading SBML should support the feature until it is actually removed.

As a matter of SBML design philosophy, the preferred approach to removing features is by deprecating them if possible. Immediate removal of SBML features is not done unless serious problems have been discovered involving those features, and keeping them would create logical inconsistencies or extremely difficult-toresolve problems. The deprecation or outright removal of features in a language, whether SBML or other, can have significant impact on backwards compatibility. Such changes are also inevitable over the course of a language's evolution. SBML must by necessity continue evolving through the experiences of its users and implementors. Eventually, some features will be deemed unhelpful despite the best intentions of the language editors to design a timeless language.

Throughout the SBML specification, removed and deprecated features are discussed in the text of the sections where the features previously appeared. Appendix G lists the changes and describes their motivations in more detail. 


\subsection{Document conventions}

In this section, we describe the conventions we use in this specification document in an effort to communicate information more effectively and consistently.

\subsubsection{Color conventions}

Throughout this document, we use coloring to carry additional information for the benefit of those viewing the document on media that can display color:

- We use red color in text and figures to indicate changes between this version of the specification (Level 2 Version 3 Release 1) and the most recent previous version of the specification (which, for the present case, is SBML Level 2 Version 2 Release 1). The changes may be either additions or deletions of text; in the case of deletions, entire sentences, paragraphs or sections are colored to indicate a change has occurred inside them.

- We use blue color in text to indicate a hyperlink from one point in this document to another. Clicking your computer's pointing device on blue-colored text will cause a jump to the section, figure, table or page to which the link refers. (Of course, this capability is only available when using electronic formats that support hyperlinking, such as PDF and HTML.)

\subsubsection{Typographical conventions for names}

The following typographical notations are used in this document to distinguish objects and data types from other kinds of entities:

AbstractClass: Abstract classes are classes that are never instantiated directly, but rather serve as parents of other classes. Their names begin with a capital letter and they are printed in a slanted, bold, sans-serif typeface. In electronic document formats, the class names are also hyperlinked to their definitions in the specification. For example, in the PDF and HTML versions of this document, clicking on the word Sbase will send the reader to the section containing the definition of this class.

Class: Names of ordinary (concrete) classes begin with a capital letter and are printed in an upright, bold, sans-serif typeface. In electronic document formats, the class names are also hyperlinked to their definitions in the specification. For example, in the PDF and HTML versions of this document, clicking on the word Species will send the reader to the section containing the definition of this class.

SomeThing, otherThing: Attributes of classes, data type names, literal XML, and generally all tokens other than SBML UML class names, are printed in an upright typewriter typeface. Primitive types defined by SBML begin with a capital letter, but unfortunately, XML Schema 1.0 does not follow any convention and primitive XML types may either start with a capital letter (e.g,. ID) or not (e.g., double).

\subsubsection{UML notation}

Previous specifications of SBML used a notation that was at one time (in the days of SBML Level 1) fairly close to UML, the Unified Modeling Language (Eriksson and Penker, 1998; Oestereich, 1999), though many details were omitted from the UML diagrams themselves. Over the years, the notation used in successive specifications of SBML grew increasingly less UML-like. Beginning with SBML Level 2 Version 3, we have completely overhauled the specification's use of UML and once again define the XML syntax of SBML using, as much as possible, proper and complete UML 1.0. We then systematically map this UML notation to XML, using XML Schema 1.0 (Biron and Malhotra, 2000; Fallside, 2000; Thompson et al., 2000) to express the overall syntax of SBML. In the rest of this section, we summarize the UML notation used in this document and explain the few embellishments needed to support transformation to XML form. A complete Schema for SBML is given in Appendix A.

We see three main advantages to using UML as a basis for defining SBML data objects. First, compared to using other notations or a programming language, the UML visual representations are generally easier to grasp by readers who are not computer scientists. Second, the notation is implementation-neutral: the 
objects can be encoded in any concrete implementation language - not just XML, but C, Java and other languages as well. Third, UML is a de facto industry standard that is documented in many resources. Readers are therefore more likely to be familiar with it than other notations.

\section{Object class definitions}

Object classes in UML diagrams are drawn as simple tripartite boxes, as shown in Figure 1 (left). UML allows for operations as well as data attributes to be defined, but SBML only uses data attributes, so all SBML class diagrams use only the top two portions of a UML class box (see the right-hand diagram of Figure 1).

\begin{tabular}{|l|l|}
\hline Class Name \\
\hline attributes \\
\hline operators
\end{tabular}$\quad$\begin{tabular}{l}
\multicolumn{1}{c|}{ ExampleClass } \\
\hline $\begin{array}{l}\text { attribute: int } \\
\text { anotherAttribute: double }\end{array}$ \\
\hline
\end{tabular}

Figure 1: (Left) The general form of a UML class diagram. (Right) Example of a class diagram of the sort seen in SBML. SBML classes never use operators, so SBML class diagrams only show the top two parts.

As mentioned above, the names of ordinary (concrete) classes begin with a capital letter and are printed in an upright, bold, sans-serif typeface. The names of attributes begin with a lower-case letter and generally use a mixed case (sometimes called "camel case") style when the name consists of multiple words. Attributes and their data types appear in the part below the class name, with one attribute defined per line. The colon character on each line separates the name of the attribute (on the left) from the type of data that it stores (on the right). The subset of data types permitted for SBML attributes is given in Section 3.1.

In the right-hand diagram of Figure 1, the symbols attribute and anotherAttribute represent attributes of the object class ExampleClass. The data type of attribute is int, and the data type of anotherAttribute is double. In the scheme used by SBML for translating UML to XML, object attributes map directly to XML attributes. Thus, in XML, ExampleClass would yield an element of the form <element attribute="42" anotherAttribute $=" 10.0 ">$.

Notice that the element name is not $<$ ExampleClass ... . Somewhat paradoxically, the name of the element is not the name of the UML class defining its structure. The reason for this may be subtle at first, but quickly becomes obvious: object classes define the form of an object's content, but a class definition by itself does not define the label or symbol referring to an instance of that content. It is this label that becomes the name of the XML element. In XML, this symbol is most naturally equated with an element name. This point will hopefully become more clear with additional examples below.

\section{Subelements}

We use UML composite aggregation to indicate a class object can have other class objects as parts. Such containment hierarchies map directly to element-subelement relationships in XML. Figure 2 gives an example.

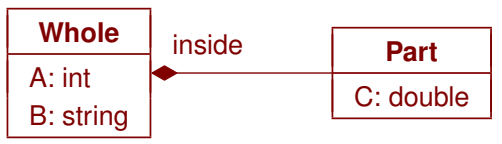

Figure 2: Example illustrating composite aggregation: the definition of one class of objects employing another class of objects in a part-whole relationship. In this particular example, an instance of a Whole class object must contain exactly one instance of a Part class object, and the symbol referring to the Part class object is inside. In XML, this symbol becomes the name of a subelement and the content of the subelement follows the definition of Part.

The line with the black diamond indicates composite aggregation, with the diamond located on the "container" side and the other end located at the object class being contained. The label on the line is the symbol used to refer to instances of the contained object, which in XML, maps directly to the name of an 
XML element. The class pointed to by the aggregation relationship (Part in Figure 2) defines the contents of that element. Thus, if we are told that some element named barney is of class Whole, the following is an example XML fragment consistent with the class definition of Figure 2:

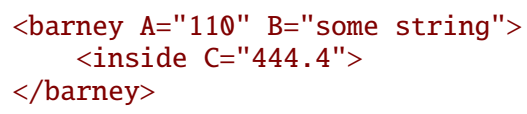

Sometimes numbers are placed above the line near the "contained" side of an aggregation to indicate how many instances can be contained. The common cases in SBML are the following: [0..*] to signify a list containing zero or more; $\left[1 .{ }^{*}\right]$ to signify a list containing at least one; and [0..1] to signify exactly zero or one. The absence of a numerical label means "exactly 1". This notation appears throughout this specification document.

\section{Inheritance}

Classes can inherit properties from other classes. Since SBML only uses data attributes and not operations, inheritance in SBML simply involves data attributes from a parent class being inherited by child classes. Inheritance is indicated by a line between two classes, with an open triangle next to the parent class; Figure 3 illustrates this. In this example, the instances of object class Child would have not only attributes C and D, but also attributes A and B. All of these attributes would be required (not optional) on instances of class Child because they are mandatory on both Parent and Child.

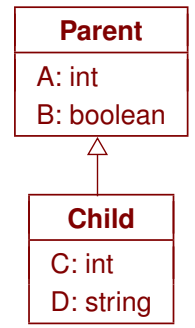

Figure 3: Inheritance.

\section{Additional notations for XML purposes}

Not everything is easily expressed in plain UML. For example, it is often necessary to indicate some constraints placed on the values of an attribute. In computer programming uses of UML, such constraints are often expressed using Object Constraint Language (OCL), but since we are most interested in the XML rendition of SBML, in this specification we use XML Schema 1.0 (when possible) as the language for expressing value constraints. Constraints on the values of attributes are written as expressions surrounded by braces $(\{\})$ after the data type declaration, as in the example of Figure 4.

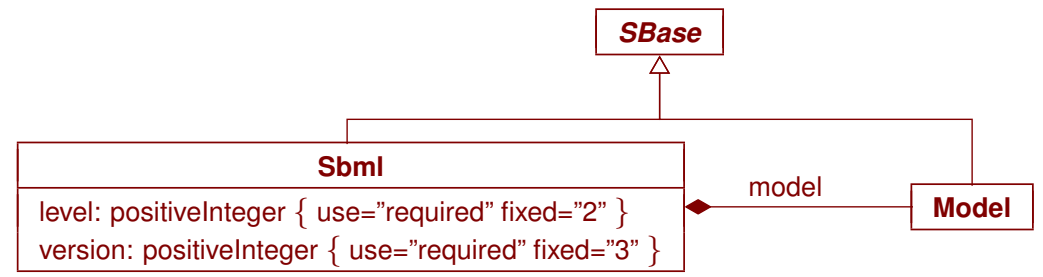

Figure 4: A more complex example definition drawing on the concepts introduced so far in this section. Both Sbml and Model are derived from SBase; further, Sbml contains a single Model object named model. Note the constraints on the values of the attributes in Sbml; they are enclosed in braces and written in XML Schema language. The particular constraints here state that both the level and version attributes must be present, and that the values are fixed as indicated.

In other situations, when something cannot be concisely expressed using a few words of XML Schema, we write constraints using English language descriptions surrounded by braces $(\{\})$. To help distinguish these from literal XML Schema, we set the English text in a slanted typeface. The text accompanying all SBML component definitions provides explanations of the constraints and any other conditions applicable to the use of the components. 
One important and confusing point that goes against the grain of XML must be highlighted: the order in which subelements appear within SBML elements is significant and must follow the order given in the corresponding object definition. This ordering is also difficult to express in plain UML, so we resort to using the approach of stating ordering requirements as constraints written in English and (again) enclosed in braces $(\{\})$. Figure 8 on page 14 gives an example of this.

The ordering restriction also holds true when a subclass inherits attributes and elements from a base class: the base class attributes and elements must occur before those introduced by the subclass.

This ordering constraint stems from aspects of XML Schema beyond our control (specifically, the need to use XML Schema's sequence construct to define the object classes). It is an occasional source of software compatibility problems, because validating XML parsers will generate errors if the ordering within an XML element does not correspond to the SBML object class definition. 


\section{Overview of SBML}

The following is an example of a simple network of biochemical reactions that can be represented in SBML:

$$
\begin{array}{ll}
S_{1} \stackrel{k_{1}\left[S_{1}\right] /\left(\left[S_{1}\right]+k_{2}\right)}{\longrightarrow} & S_{2} \\
S_{2} \stackrel{k_{3}\left[S_{2}\right]}{\longrightarrow} & S_{3}+S_{4}
\end{array}
$$

In this particular set of chemical equations above, the symbols in square brackets (e.g., "[ $\left.S_{1}\right]$ ") represent concentrations of molecular species, the arrows represent reactions, and the formulas above the arrows represent the rates at which the reactions take place. (And while this example uses concentrations, it could equally have used other measures such as molecular counts.) Broken down into its constituents, this model contains a number of components: reactant species, product species, reactions, reaction rates, and parameters in the rate expressions. To analyze or simulate this network, additional components must be made explicit, including compartments for the species, and units on the various quantities.

SBML allows models of arbitrary complexity to be represented. Each type of component in a model is described using a specific type of data object that organizes the relevant information. The top level of an SBML model definition consists of lists of these components, with every list being optional:

$$
\begin{array}{ll}
\text { beginning of model definition } & \\
\text { list of function definitions (optional) } & \text { (Section 4.3) } \\
\text { list of unit definitions (optional) } & \text { (Section 4.4) } \\
\text { list of compartment types (optional) } & \text { (Section 4.5) } \\
\text { list of species types (optional) } & \text { (Section 4.6) } \\
\text { list of compartments (optional) } & \text { (Section 4.7) } \\
\text { list of species (optional) } & \text { (Section 4.8) } \\
\text { list of parameters (optional) } & \text { (Section 4.9) } \\
\text { list of initial assignments (optional) } & \text { (Section 4.10) } \\
\text { list of rules (optional) } & \text { (Section 4.11) } \\
\text { list of constraints (optional) } & \text { (Section 4.12) } \\
\text { list of reactions (optional) } & \text { (Section 4.13) } \\
\text { list of events (optional) } & \text { (Section 4.14) } \\
\text { end of model definition } &
\end{array}
$$

(Section 4.3)
(Section 4.4)

(Section 4.5)

(Section 4.6)

(Section 4.7)

(Section 4.8)

(Section 4.9)

(Section 4.10)

(Section 4.11)

(Section 4.12)

(Section 4.13)

(Section 4.14)

The meaning of each component is as follows:

Function definition: A named mathematical function that may be used throughout the rest of a model.

Unit definition: A named definition of a new unit of measurement, or a redefinition of an existing SBML default unit. Named units can be used in the expression of quantities in a model.

Compartment Type: A type of location where reacting entities such as chemical substances may be located.

Species type: A type of entity that can participate in reactions. Typical examples of species types include ions such as $\mathrm{Ca}^{2+}$, molecules such as glucose or ATP, and more.

Compartment: A well-stirred container of a particular type and finite size where species may be located. A model may contain multiple compartments of the same compartment type. Every species in a model must be located in a compartment.

Species: A pool of entities of the same species type located in a specific compartment.

Parameter: A quantity with a symbolic name. In SBML, the term parameter is used in a generic sense to refer to named quantities regardless of whether they are constants or variables in a model. SBML Level 2 provides the ability to define parameters that are global to a model as well as parameters that are local to a single reaction. 
Initial Assignment: A mathematical expression used to determine the initial conditions of a model. This type of object can only be used to define how the value of a variable can be calculated from other values and variables at the start of simulated time.

Rule: A mathematical expression added to the set of equations constructed based on the reactions defined in a model. Rules can be used to define how a variable's value can be calculated from other variables, or used to define the rate of change of a variable. The set of rules in a model can be used with the reaction rate equations to determine the behavior of the model with respect to time. The set of rules constrains the model for the entire duration of simulated time.

Constraint: A means of detecting out-of-bounds conditions during a dynamical simulation and optionally issuing diagnostic messages. Constraints are defined by an arbitrary mathematical expression computing a true/false value from model variables, parameters and constants. An SBML constraint applies at all instants of simulated time; however, the set of constraints in model should not be used to determine the behavior of the model with respect to time.

Reaction: A statement describing some transformation, transport or binding process that can change the amount of one or more species. For example, a reaction may describe how certain entities (reactants) are transformed into certain other entities (products). Reactions have associated kinetic rate expressions describing how quickly they take place.

Event: A statement describing an instantaneous, discontinuous change in a set of variables of any type (species quantity, compartment size or parameter value) when a triggering condition is satisfied.

A software package can read an SBML model description and translate it into its own internal format for model analysis. For example, a package might provide the ability to simulate the model by constructing differential equations representing the network and then perform numerical time integration on the equations to explore the model's dynamic behavior. By supporting SBML as an input and output format, different software tools can all operate on an identical external representation of a model, removing opportunities for errors in translation and assuring a common starting point for analyses and simulations. 


\section{Preliminary definitions and principles}

This section covers certain concepts and constructs that are used repeatedly in the rest of SBML Level 2.

\subsection{Primitive data types}

Most primitive types in SBML are taken from the data types defined in XML Schema 1.0 (Biron and Malhotra, 2000; Fallside, 2000; Thompson et al., 2000). A few other primitive types are defined by SBML itself. What follows is a summary of the XML Schema types and the definitions of the SBML-specific types. Note that while we have tried to provide accurate and complete summaries of the XML Schema types, the following should not be taken to be normative definitions of these types. Readers should consult the XML Schema 1.0 specification for the normative definitions of the XML types used by SBML.

\subsubsection{Type string}

The XML Schema 1.0 type string is used to represent finite-length strings of characters. The characters permitted to appear in XML Schema string include all Unicode characters (Unicode Consortium, 1996) except for two delimiter characters, 0xFFFE and 0xFFFF (Biron and Malhotra, 2000). In addition, the following quoting rules specified by XML for character data (Bray et al., 2000) must be obeyed:

- The ampersand (\&) character must be escaped using the entity \&amp; .

- The apostrophe (') and quotation mark (") characters must be escaped using the entities \&apos; and \&quot; , respectively, when those characters are used to delimit a string attribute value.

Other XML built-in character or entity references, e.g., \&1t; and \&x1A; , are permitted in strings.

\subsubsection{Type boolean}

The XML Schema 1.0 type boolean is used as the data type for SBML object attributes that represent binary true/false values. XML Schema 1.0 defines the possible literal values of boolean as the following: "true", "false", "1", and " 0 ". The value "1" maps to "true" and the value " 0 " maps to "false".

Note that there is a discrepancy between the value spaces of type boolean as defined by XML Schema 1.0 and MathML: the latter uses only "true" and "false" to represent boolean values and "0" and "1" are interpreted as numbers. Software tools should take care to not to use "0" and "1" as boolean values in MathML expressions. See further discussion in Section 3.4.4.

\subsubsection{Type int}

The XML Schema 1.0 type int is used to represent decimal integer numbers in SBML. The literal representation of an int is a finite-length sequence of decimal digit characters with an optional leading sign (" +" or "-"). If the sign is omitted, "+" is assumed. The value space of int is the same as a standard 32-bit signed integer in programming languages such as C, i.e., 2147483647 to -2147483648.

\subsubsection{Type positiveInteger}

The XML Schema 1.0 type positiveInteger is used to represent nonzero, nonnegative, decimal integers: i.e., $1,2,3, \ldots$ The literal representation of an integer is a finite-length sequence of decimal digit characters, optionally preceded by a positive sign ("+"). There is no restriction on the absolute size of positiveInteger values in XML Schema; however, the only situations where this type is used in SBML involve very lownumbered integers. Consequently, applications may safely treat positiveInteger as unsigned 32-bit integers.

\subsubsection{Type double}

The XML Schema 1.0 type double is the data type of floating point numerical quantities in SBML. It is restricted to IEEE double-precision 64-bit floating point type IEEE 754-1985. The value space of double consists of (a) the numerical values $m \times 2^{x}$, where $m$ is an integer whose absolute value is less than $2^{53}$, 
and $x$ is an integer between -1075 and 970 , inclusive, (b) the special value positive infinity (INF), (c) the special value negative infinity (-INF), and (d) the special value not-a-number (NaN). The order relation on the values is the following: $x<y$ if and only if $y-x$ is positive for values of $x$ and $y$ in the value space of double. Positive infinity is greater than all other values other than NaN. NaN is equal to itself but is neither greater nor less than any other value in the value space. (Software implementors should consult the XML Schema 1.0 definition of double for additional details about equality and relationships to IEEE 754-1985.)

The general form of double numbers is " $x \mathbf{e} y$ ", where $x$ is a decimal number (the mantissa), "e" is a separator character, and $y$ is an exponent; the meaning of this is " $x$ multiplied by 10 raised to the power of $y$ ", i.e., $x \times 10^{y}$. More precisely, a double value consists of a mantissa with an optional leading sign ("+" or "_"), optionally followed by the character $\mathrm{E}$ or e followed by an integer (the exponent). The mantissa must be a decimal number: an integer optionally followed by a period (.) optionally followed by another integer. If the leading sign is omitted, "+" is assumed. An omitted E or e and exponent means that a value of 0 is assumed for the exponent. If the $\mathrm{E}$ or e is present, it must be followed by an integer or an error results. The integer acting as an exponent must consist of a decimal number optionally preceded by a leading sign ("+" or "-"). If the sign is omitted, "+" is assumed. The following are examples of legal literal double values:

$$
-1 \mathrm{E} 4, \quad+4, \quad 234.234 \mathrm{e} 3, \quad 6.02 \mathrm{E}-23, \quad 0.3 \mathrm{e}+11, \quad 2, \quad 0, \quad-0, \quad \text { INF, } \quad-\text { INF, NaN }
$$

As described in Section 3.4, SBML uses a subset of the MathML 2.0 standard (W3C, 2000b) for expressing mathematical formulas in XML. This is done by stipulating that the MathML language be used whenever a mathematical formula must be written into an SBML model. Doing this, however, requires facing two problems: first, the syntax of numbers in scientific notation ("e-notation") is different in MathML from that just described for double, and second, the value space of integers and floating-point numbers in MathML is not defined in the same way as in XML Schema 1.0. We elaborate on these issues in Section 3.4.2; here we summarize the solution taken in SBML. First, within MathML, the mantissa and exponent of numbers in "e-notation" format must be separated by one $<$ sep/> element. This leads to numbers of the form $<\mathrm{cn}$ type="e-notation" $>2<\mathrm{sep} />-5</ \mathrm{cn}>$. Second, SBML stipulates that the representation of numbers in MathML expressions obey the same restrictions on values as defined for types double and int (Section 3.1.3).

\subsubsection{Type ID}

The XML Schema 1.0 type ID is identical to the XML 1.0 type ID. The literal representation of this type consists of strings of characters restricted as summarized in Figure 5.

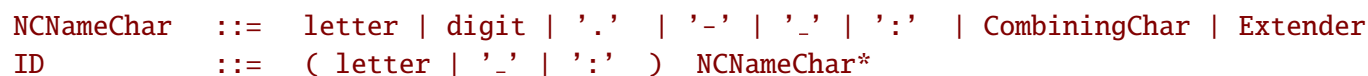

Figure 5: Type ID expressed in the variant of BNF used by the XML 1.0 specification (Bray et al., 2004). The characters ( and ) are used for grouping, the character * indicates "zero or more times", and the character I indicates "or". The production letter consists of the basic upper and lower case alphabetic characters of the Latin alphabet along with a large number of related characters defined by Unicode 2.0; similarly, the production digit consists of the numerals 0 . . 9 along with related Unicode 2.0 characters. The CombiningChar production is a list of characters that add such things as accents to the preceding character. (For example, the Unicode character \# $030 \mathrm{~A}$ when combined with 'a' produces 'å') The Extender production is a list of characters that extend the shape of the preceding character. Please consult the XML 1.0 specification (Bray et al., 2004) for the complete definitions of letter, digit, CombiningChar, and Extender.

In SBML, type ID is the data type of the metaid attribute on Sbase, described in Section 3.2. An important aspect of ID is the XML requirement that a given value of ID must be unique throughout an XML document. All data values of type ID are considered to reside in a single common global namespace spanning the entire XML document, regardless of the attribute where type ID is used and regardless of the level of nesting of the objects (or XML elements).

\subsubsection{Type SId}

The type SId is the type of the id attribute found on the majority of SBML components. SId is a data type derived from the basic XML type string, but with restrictions about the characters permitted and the sequences in which those characters may appear. The definition is shown in Figure 6 on the next page. 


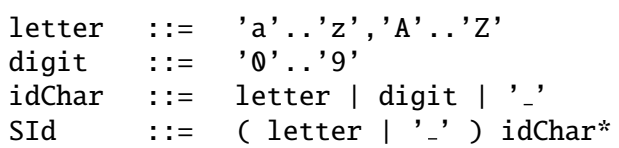

Figure 6: The definition of the type SId. (Please see the caption of Figure 5 for an explanation of the notation.)

The equality of SId values is determined by an exact character sequence match; i.e., comparisons of these identifiers must be performed in a case-sensitive manner. This applies to all uses of SId.

The SId is purposefully not derived from the XML ID type (Section 3.1.6). Using XML's ID would force all SBML identifiers to exist in a single global namespace, which would affect not only the form of local parameter definitions but also future SBML extensions for supporting model/submodel composition. Further, the use of the ID type for SBML identifiers would have limited utility because MathML 2.0 ci elements are not of the type IDREF (see Section 3.4). Since the IDREF/ID linkage cannot be exploited in MathML constructs, the utility of the XML ID type is greatly reduced. Finally, unlike ID, SId does not include Unicode character codes; the identifiers are plain text.

\subsubsection{Type UnitSId}

The type UnitSId is derived from SId (Section 3.1.7) and has identical syntax. The UnitSId type is used as the data type for the identifiers of units (Section 4.4.1) and for references to unit identifiers in SBML objects. The purpose of having a separate data type for such identifiers is enable the space of possible unit identifier values to be separated from the space of all other identifier values in SBML. The equality of UnitSId values is determined by an exact character sequence match; i.e., comparisons of these identifiers must be performed in a case-sensitive manner.

A number of reserved symbols are defined in the space of values of UnitSId. These reserved symbols are the list of base unit names defined in Table 1 on page 37, and the SBML built-in units "substance", "volume", "area", "length", and "time" listed in Table 2 on page 41. These symbols and their use is described in Section 4.4.

\subsubsection{Type SBOTerm}

The type SBOTerm is used as the data type of the attribute sboTerm on Sbase. The type consists of strings of characters matching the restricted pattern described in Figure 7.

$$
\begin{aligned}
& \text { digit } \quad::=\text { 'O'..' ', } \\
& \text { SBOTerm ::= 'SBO:' digit digit digit digit digit digit digit }
\end{aligned}
$$

Figure 7: The definition of SBOTerm. The SBOTerm type consists of strings beginning with SBO: and followed by seven decimal digits. (Please see the caption of Figure 5 for an explanation of the notation.)

Examples of valid string values of type SBOTerm are "SBO:0000014" and "SB0:0003204". These values are meant to be the identifiers of terms from an ontology whose vocabulary describes entities and processes in computational models. Section 5 provides more information about the ontology and principles for the use of these terms in SBML models.

\subsection{Type SBase}

Nearly every object composing an SBML Level 2 model definition has a specific data type that is derived directly or indirectly from a single abstract type called Sbase. In addition to serving as the parent class for most other classes of objects in SBML, this base type is designed to allow a modeler or a software package to attach arbitrary information to each major element or list in an SBML model. The definition of Sbase is presented in Figure 8 on the following page.

Sbase contains two attributes and two subelements, all of which are optional: metaid, sboTerm, notes and annotation. These are discussed separately in the following subsections. 


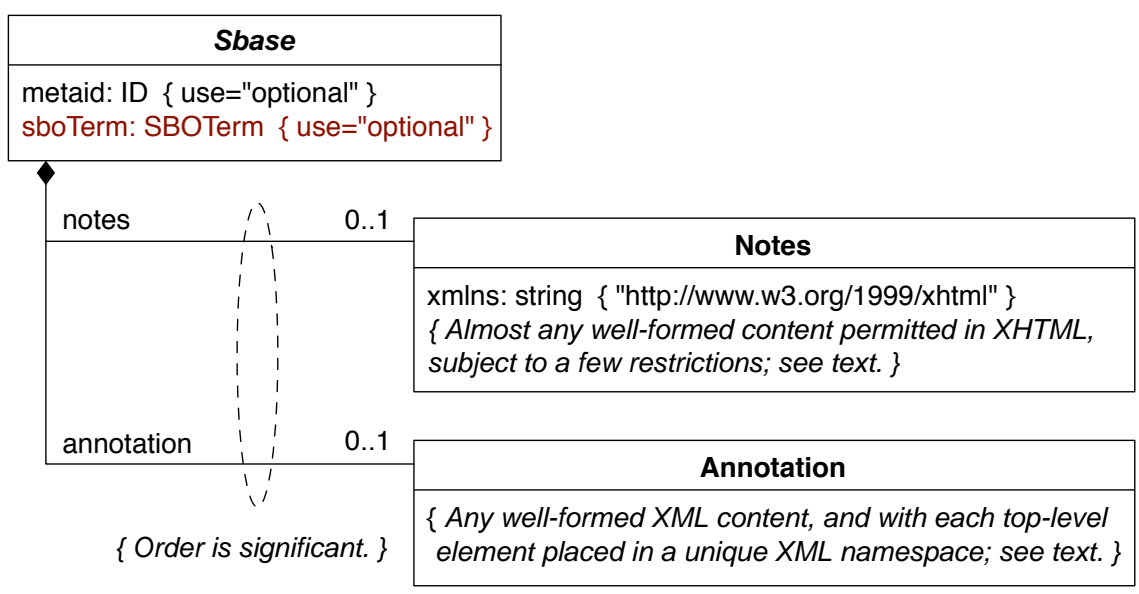

Figure 8: The definition of Sbase. Please refer to Section 1.4 for a summary of the UML notation used here. Note that the order of appearance of subelements notes and annotation is significant in instances of objects derived from SBase: notes must always come before annotation. (This requirement arises from XML Schema 1.0.)

\subsubsection{The metaid attribute}

The metaid attribute is present for supporting metadata annotations using RDF (Resource Description Format; Lassila and Swick, 1999). It has a data type of XML ID (the XML identifier type; see Section 3.1.6), which means each metaid value must be globally unique within an SBML file. The metaid value serves to identify a model component for purposes such as referencing that component from metadata placed within annotation elements (see Section 3.2.4). Such metadata can use RDF description elements, in which an RDF attribute called "rdf:about" points to the metaid identifier of an object defined in the SBML model. This topic is discussed in greater detail in Section 6.

\subsubsection{The sboTerm attribute}

The attribute called sboTerm is provided on Sbase to support the use of the Systems Biology Ontology (SBO; see Section 5). When a value is given to this attribute, it must conform to the data type SBOTerm (Sections 3.1.9). SBO terms are a type of optional annotation, and each different class of SBML object derived from Sbase imposes its own requirements about the values permitted for sboTerm. Specific details on the permitted values are provided with the definitions of SBML classes throughout this specification document, and a broader discussion is provided in Section 5 .

\subsubsection{The notes element}

The element notes in Sbase is a container for XHTML 1.0 (Pemberton et al., 2002) content. It is intended to serve as a place for storing optional information intended to be seen by humans. An example use of the notes element would be to contain formatted user comments about the model element in which the notes element is enclosed. Every object derived directly or indirectly from type Sbase can have a separate value for notes, allowing users considerable freedom when adding comments to their models.

XHTML 1.0 is simply a formulation of HTML 4 in XML 1.0. This means the full power of HTML formatting is available for use in notes content. The intention behind requiring XHTML (rather than, for example, plain HTML or plain text) for notes content is to balance several conflicting goals: (1) choosing a format for notes that is compatible with the XML form of SBML (plain HTML would not be); (2) supporting an international formatting standard so that users have more control over the appearance of notes and can predict to some degree how their notes will be displayed in different tools and environments (which argues against using plain-text notes); and (3) achieving these goals using an approach that is hopefully easy enough for software developers to support using off-the-shelf programming libraries. It is worth noting in passing that the requirement for XHTML does not prevent users from entering plain-text content with simple space/tab/newline formatting: it merely requires using the standard $<$ pre $>\ldots<$ pre $>$ element of $(\mathrm{X}) \mathrm{HTML}$. 
Modern libraries for displaying and editing (X)HTML content are commonly available in contemporary software programming environments, and software developers may wish to avail themselves of these facilities rather than implementing their own XHTML support systems.

\section{$X M L$ namespace requirements for notes}

The XML content of notes elements must declare the use of the XHTML XML namespace. This can be done in multiple ways. One way is to place a namespace declaration for the appropriate namespace URI (which is http://www.w3.org/1999/xhtml) on the top-level Sbml object (see Section 4.1) and then reference the namespace in the notes content using a prefix. The following example illustrates this approach:

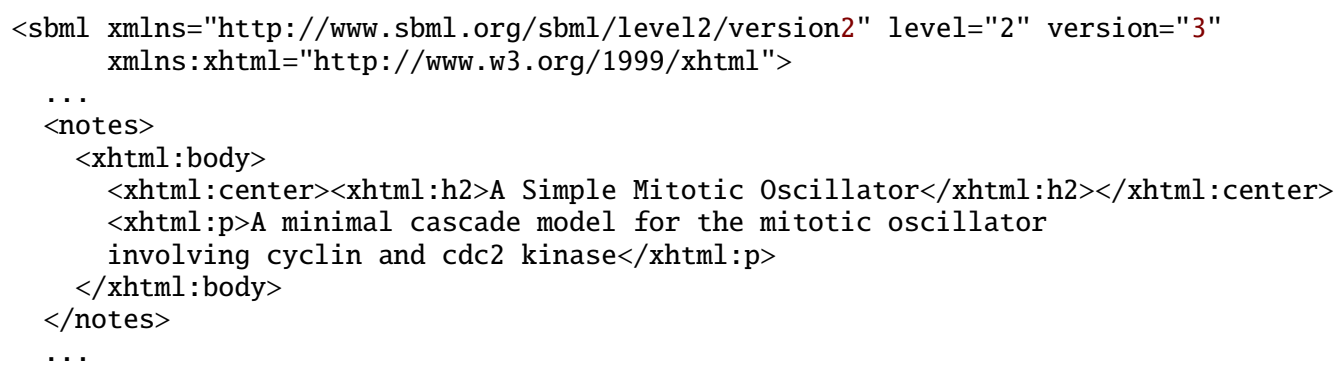

Another approach is to declare the XHTML namespace within the notes content itself, as in the following example:

$\ldots$

The xmlns="http://www .w3.org/1999/xhtml" declaration on body as shown above changes the default XML namespace within it, such that all of its content is by default in the XHTML namespace. This is a particularly convenient approach because it obviates the need to prefix every element with a namespace prefix (i.e., xhtml: in the previous case). Other approaches are also possible.

\section{The content of notes}

SBML does not require the content of notes to be any particular XHTML element; the content can be almost any well-formed XHTML content. There are only two simple restrictions. The first restriction comes from the requirements of XML: the notes element must not contain an XML declaration nor a DOCTYPE declaration. That is, notes must not contain

$<$ ?xml version="1.0" encoding="UTF-8"?>

nor (where the following is only one specific example of a DOCTYPE declaration)

<!DOCTYPE html PUBLIC "-//W3C//DTD XHTML 1.0 Strict//EN"

"http://www.w3.org/TR/xhtml1/DTD/xhtml1-strict.dtd">

The second restriction is intended to balance freedom of content with the complexity of implementing software that can interpret the content. The content of notes in SBML can consist only of the following possibilities:

1. A complete XHTML document (minus the XML and DOCTYPE declarations, of course), that is, XHTML content beginning with the html tag. The following is an example skeleton: 


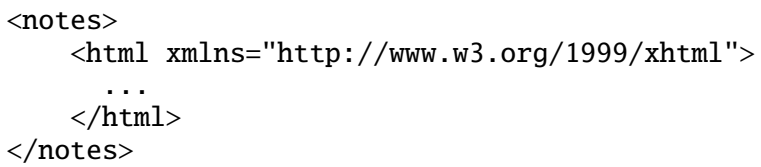

2. The body element from an XHTML document. The following is an example skeleton:

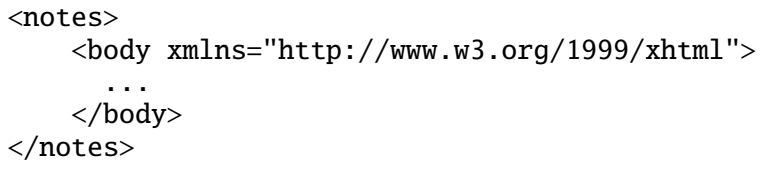

3. Any XHTML content that would be permitted within a body element. If this consists of multiple elements, each one must declare the XML namespace separately. The following is an example fragment:

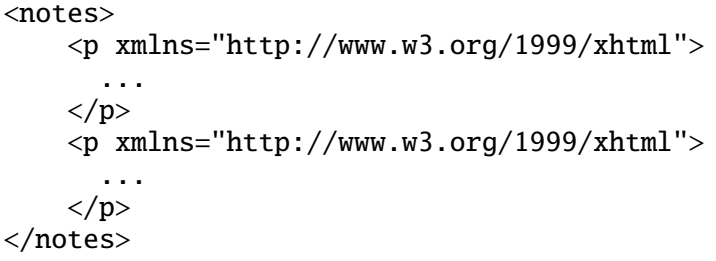

Another way to summarize the restrictions above is simply to say that the content of an SBML notes element can be only be a complete html element, a body element, or whatever is permitted inside a body element. In practice, this does not limit in any meaningful way what can be placed inside a notes element; for example, if an application or modeler wants to put a complete XHTML page, including a head element, it can be done by putting in everything starting with the html container. However, the restrictions above do make it somewhat simpler to write software that can read and write the notes content. Appendix F describes one possible approach to doing just that.

\subsubsection{The annotation element}

Whereas the notes element described above is a container for content to be shown directly to humans, the annotation element is a container for optional software-generated content not meant to be shown to humans. Every object derived from Sbase can have its own value for annotation. The element's content type is XML type any, allowing essentially arbitrary well-formed XML data content. SBML places only a few restrictions on the organization of the content; these are intended to help software tools read and write the data as well as help reduce conflicts between annotations added by different tools.

\section{The use of XML namespaces in annotation}

At the outset, software developers should keep in mind that multiple software tools may attempt to read and write annotation content. To reduce the potential for collisions between annotations written by different applications, SBML Level 2 Version 3 stipulates that tools must use XML namespaces (Bray et al., 1999) to specify the intended vocabulary of every annotation. The application's developers must choose a URI (Universal Resource Identifier; Harold and Means 2001; W3C 2000a) reference that uniquely identifies the vocabulary the application will use, and a prefix string for the annotations. Here is an example. Suppose an application uses the URI http://www.mysim.org/ns and the prefix mysim when writing annotations related to screen layout. The content of an annotation might look like the following:

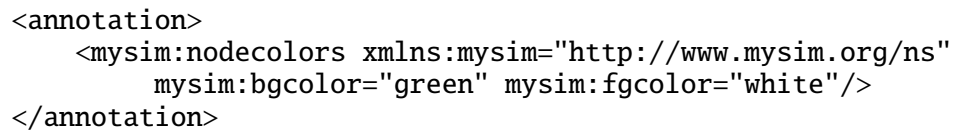

In this particularly simple example, the content consists of a single XML element (nodecolors) with two attributes (bgcolor, fgcolor), all of which are prefixed by the string mysim. (Presumably this particular content would have meaning to the hypothetical application in question.) The content in this particular 
example is small, but it should be clear that there could easily have been an arbitrarily large amount of data placed inside the mysim:nodecolors element.

The key point of the example above is that application-specific annotation data is entirely contained inside a single top-level element within the SBML annotation container. SBML Level 2 Version 3 places the following restrictions on annotations:

- Within a given SBML annotation element, there can only be one top-level element using a given namespace. An annotation element can contain multiple top-level elements but each must be in a different namespace.

- No top-level element in an annotation may use an SBML XML namespace, either explicitly by referencing one of the SBML XML namespace URIs or implicitly by failing to specify any namespace on the annotation. As of SBML Level 2 Version 3, the defined SBML namespaces are the following URIs:

- http://www.sbml.org/sbml/level1

- http://www.sbml.org/sbml/level2

- http://www. sbml .org/sbml/level2/version2

- http://www.sbml.org/sbml/level2/version3

- The ordering of top-level elements within a given annotation element is not significant. An application should not expect that its annotation content appears first in the annotation element, nor in any other particular location. Moreover, the ordering of top-level annotation elements may be changed by different applications as they read and write the same SBML file.

The use of XML namespaces in this manner is intended to improve the ability of multiple applications to place annotations on SBML model elements with reduced risks of interference or name collisions. Annotations stored by different simulation packages can therefore coexist in the same model definition. The rules governing the content of annotation elements are designed to enable applications to easily add, change, and remove their annotations from SBML elements while simultaneously preserving annotations inserted by other applications when mapping SBML from input to output.

As a further simplification for developers of software and to improve software interoperability, applications are only required to preserve other annotations (i.e., annotations they do not recognize) when those annotations are self-contained entirely within annotation, complete with namespace declarations. The following is an example:

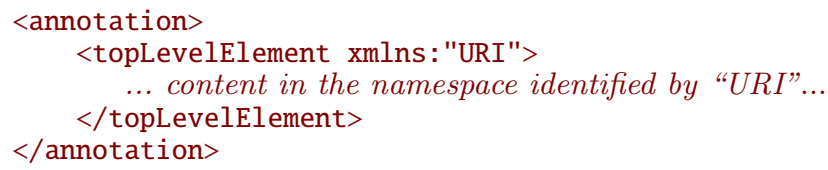

Some more examples hopefully will make these points more clear. The next example is invalid because it contains a top-level element in the SBML XML namespace - this happens because no namespace is declared for the <cytoplasm> element, which means by default it falls into the enclosing SBML namespace:

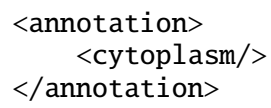

The following example is also invalid, this time because it contains two top-level elements using the same XML namespace. Note that it does not matter that these are two different top-level elements (<nodecolors $>$ and <textcolors $>$ ); what matters is that these separate elements are both in the same namespace rather than having been collected and placed inside one overall container element for that namespace.

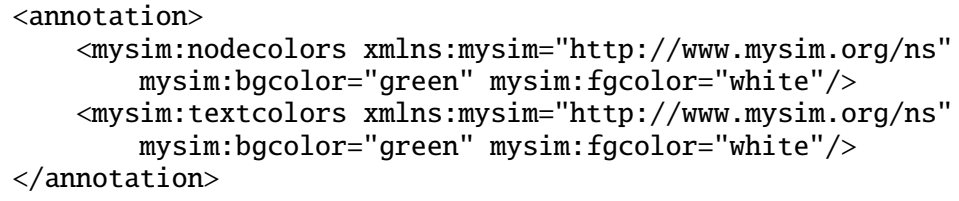


On the other hand, the following example is valid:

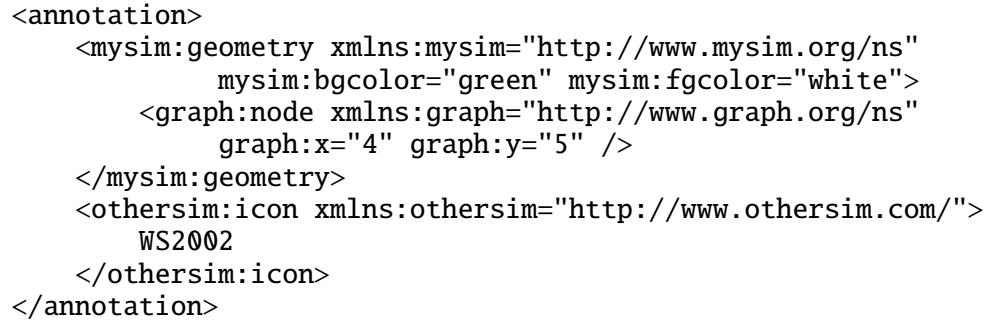

For completeness, we note that annotations can legally be empty:

<annotation />

It is worth keeping in mind that although XML namespace names must be URIs, they are (like all XML namespace names) not required to be directly usable in the sense of identifying an actual, retrieval document or resource on the Internet (Bray et al., 1999). URIs such as http://www.mysim.org/ may appear as though they are (e.g.,) Internet addresses, but there are not the same thing. This style of URI strings, using a domain name and other parts, is only a simple and commonly-used way of creating a unique name string.

Finally, note that the namespaces being referred to here are XML namespaces specifically in the context of the annotation element on Sbase. The namespace issue here is unrelated to the namespaces discussed in Section 3.3.1 in the context of component identifiers in SBML.

\section{Content of annotations and implications for software tools}

The annotation element in the definition of Sbase exists in order that software developers may attach optional application-specific data to the elements in an SBML model. However, it is important that this facility not be misused. In particular, it is critical that data essential to a model definition or that can be encoded in existing SBML elements is not stored in annotation. Parameter values, functional dependencies between model elements, etc., should not be recorded as annotations. It is crucial to keep in mind the fact that data placed in annotations can be freely ignored by software applications. If such data affects the interpretation of a model, then software interoperability is greatly impeded.

Here are examples of the kinds of data that may be appropriately stored in annotation: (a) information about the graphical layout of model components; (b) application-specific processing instructions that do not change the essential meaning of a model; (c) identification information for cross-referencing components in a model with items in a data resource such as a database; and (d) information about the model that cannot be readily encoded in existing SBML elements.

\section{Standardized format for certain classes of annotations}

For case (c) above (i.e., cross-references between model components and data resources, SBML Level 2 Version 3 recommends a standard format for use within annotation elements. It should be used in preference to proprietary syntaxes to maximize the likelihood that multiple software tools will converge on the same syntax for this kind of information. The recommended scheme is described in Section 6 .

\subsection{The id and name attributes on SBML components}

As will become apparent below, most objects in SBML include two common attributes: id and name. These attributes are not defined on Sbase (as explained in Section 3.3.3 below), but where they do appear, the common rules of usage described below apply.

\subsubsection{The id attribute and identifier scoping}

The id attribute is mandatory on most objects in SBML. It is used to identify a component within the model definition. Other SBML objects can refer to the component using this identifier. The data type of id is always either Sid (Section 3.1.7) or UnitSId (Section 3.1.8), depending on the object in question. 
A model can contain a large number of components representing different parts. This leads to a problem in deciding the scope of an identifier: in what contexts does a given identifier $X$ represent the same thing? The approaches used in existing simulation packages tend to fall into two categories which we may call global and local. The global approach places all identifiers into a single global space of identifiers, so that an identifier $X$ represents the same thing wherever it appears in a given model definition. The local approach places symbols in separate identifier namespaces, depending on the context, where the context may be, for example, individual reaction rate expressions. The latter approach means that a user may use the same identifier $X$ in different rate expressions and have each instance represent a different quantity.

The fact that different simulation programs may use different rules for identifier resolution poses a problem for the exchange of models between simulation tools. Without careful consideration, a model written out in SBML format by one program may be misinterpreted by another program. SBML Level 2 must therefore include a specific set of rules for treating identifiers and their scopes.

The scoping rules in SBML Level 2 are relatively straightforward and are intended to avoid this problem with a minimum of requirements on the implementation of software tools:

- The identifier (i.e., the value of the attribute id) of every FunctionDefinition, CompartmentType, SpeciesType, Compartment, Species, Parameter, Reaction, SpeciesReference, ModifierSpeciesReference, Event, and Model, must be unique across the set of all such identifiers in the model. This means, for example, that a reaction and a species definition cannot both have the same identifier.

- The identifier of every UnitDefinition must be unique across the set of all such identifiers in the model. However, unit identifiers live in a separate space of identifiers from other identifiers in the model, by virtue of the fact that the data type of unit identifiers is UnitSId (Section 3.1.8) and not SId.

- Each Reaction instance (see Section 4.13) establishes a separate private local space for local Parameter identifiers. Within the definition of that reaction, local parameter identifiers override (shadow) identical identifiers outside of that reaction. Of course, the corollary of this is that local parameters inside a Reaction object instance are not visible to other objects outside of that reaction.

The set of rules above can enable software packages using either local or global identifier spaces for parameters to exchange SBML model definitions. Software systems using local identifiers for parameters internally should, in principle, be able to accept SBML model definitions without needing to change component identifiers. Environments using a common global space of identifiers for parameters internally can perform manipulations of the identifiers of local parameters within reaction definitions to avoid identifier collisions.

The guidelines described here will hopefully provide a clean transition path to future levels of SBML, when submodels are introduced (Section 8.1). Submodels will provide the ability to compose one model from a collection of other models. This capability will have to be built on top of SBML Level 2's namespace organization. A straightforward approach to handling namespaces is to make each submodel's space be private. The rules governing identifier scoping within a submodel can simply be the Level 2 namespace rule described here, with each submodel having its own (to itself, global) namespace.

\subsubsection{The name attribute}

In contrast to the id attribute, the name attribute is optional and is not intended to be used for crossreferencing purposes within a model. Its purpose instead is to provide a human-readable label for the component. The data type of name is the type string defined in XML Schema (Biron and Malhotra, 2000; Thompson et al., 2000) and discussed further in Section 3.1. SBML imposes no restrictions as to the content of name attributes beyond those restrictions defined by the string type in XML Schema.

The recommended practice for handling name is as follows. If a software tool has the capability for displaying the content of name attributes, it should display this content to the user as a component's label instead of the component's id. If the user interface does not have this capability (e.g., because it cannot display or use special characters in symbol names), or if the name attribute is missing on a given component, then the user interface should display the value of the id attribute instead. (Script language interpreters are especially likely to display id instead of name.) 
As a consequence of the above, authors of systems that automatically generate the values of id attributes should be aware some systems may display the id's to the user. Authors therefore may wish to take some care to have their software create id values that are: (a) reasonably easy for humans to type and read; and (b) likely to be meaningful, e.g., the id attribute is an abbreviated form of the name attribute value.

An additional point worth mentioning is although there are restrictions on the uniqueness of id values (see Section 3.3.1 above), there are no restrictions on the uniqueness of name values in a model. This allows software packages leeway in assigning component identifiers.

\subsubsection{Why id and name are not defined on SBase}

Although many SBML components feature id and name, these attributes are purposefully not defined on Sbase. There are several reasons for this.

- The presence of an SBML identifier attribute (id) necessarily requires specifying scoping rules for the corresponding identifiers. However, the Sbase abstract type is used as the basis for defining components whose scoping rules are in some cases different from each other. (See Section 3.3.1 for more details). If Sbase were to have an id attribute, then the specification of Sbase would need a default scoping rule and this would then have to be overloaded on derived classes that needed different scoping. This would make the SBML specification even more complex.

- Identifier are optional on some SBML components and required on most others. If id were defined as optional on Sbase, most component classes would separately have to redefine id as being mandatoryhardly an improvement over the current arrangement. Conversely, if id were defined as mandatory on Sbase, it would prevent it from being optional on components where it is currently optional.

- The Sbase abstract type is used as the base type for certain objects such as Sbml, AssignmentRule, etc., which do not have identifiers because these components do not need to be referenced by other components. If Sbase had a mandatory id attribute, all objects of these other types in a model would then need to be assigned unique identifiers. Similarly, because Sbase is the base type of the list0f_ lists, putting id on Sbase would require all of these lists in a model to be given identifiers. This would be a needless burden on software developers, tools, and SBML users, requiring them to generate and store additional identifiers for objects that never need them.

- Sbase does not have a name simply because such an attribute is always paired with an id. Without id on Sbase, it does not make sense to have name.

\subsection{Mathematical formulas in SBML Level 2}

Mathematical expressions in SBML Level 2 are represented using MathML 2.0 (W3C, 2000b). MathML is an international standard for encoding mathematical expressions using XML. There are two principal facets of MathML, one for encoding content (i.e., the semantic interpretation of a mathematical expression), and another for encoding presentation or display characteristics. SBML only makes direct use of a subset of the content portion of MathML. By borrowing a separately-developed XML standard, we can avoid having to define a specialized syntax for mathematical expressions in SBML and simultaneously leverage existing intellectual and technological work already done in the MathML community. However, it is not possible to produce a completely smooth and conflict-free interface between MathML and other standards used by SBML (in particular, XML Schema). Two specific issues and their resolutions are discussed in Sections 3.4.2.

The XML namespace URI for all MathML elements is http://www.w3.org/1998/Math/MathML. Everywhere MathML content is allowed in SBML, the MathML elements must be properly placed within the MathML 2.0 namespace. In XML, this can be accomplished in a number of ways, and the examples throughout this specification illustrate the use of this namespace and MathML in SBML. Please refer to the W3C document by Bray et al. (1999) for more technical information about using XML namespaces. 


\subsubsection{Subset of MathML used in SBML Level 2}

The subset of MathML 2.0 elements used in SBML Level 2 is similar to that used by CellML (Hedley et al., 2001), another model definition language with similar goals as SBML. The subset of MathML elements used in SBML is listed below:

- token: cn, ci, csymbol, sep

- general: apply, piecewise, piece, otherwise, lambda (the last is restricted to use in FunctionDefinition)

- relational operators: eq, neq, gt, lt, geq, leq

- arithmetic operators: plus, minus, times, divide, power, root, abs, exp, ln, log, floor, ceiling, factorial

- logical operators: and, or, xor, not

- qualifiers: degree, bvar, logbase

- trigonometric operators: sin, cos, tan, sec, csc, cot, sinh, cosh, tanh, sech, csch, coth, arcsin, arccos, arctan, arcsec, arccsc, arccot, arcsinh, arccosh, arctanh, arcsech, arccsch, arccoth

- constants: true, false, notanumber, pi, infinity, exponentiale

- annotation: semantics, annotation, annotation-xml

The inclusion of logical operators, relational operators, piecewise, piece, and otherwise elements facilitates the encoding of discontinuous expressions. Note that MathML elements for representing partial differential calculus are not included. We anticipate that the requirements for partial differential calculus will be addressed in proposals for future SBML geometry representations (see Section 8.1).

As defined by MathML 2.0, the semantic interpretation of the mathematical functions listed above follows the definitions of the functions laid out by Abramowitz and Stegun (1977) and Zwillinger (1996). Readers are directed to these sources and the MathML specification for information about such things as which principal values of the inverse trigonometric functions to use.

Software authors should take particular note of the MathML semantics of the N-ary operators plus, times, and, or and xor, when they are used with different numbers of arguments. The MathML specification (W3C, 2000b) appendix C.2.3 describes the semantics for these operators with zero, one, and more arguments.

The following are the only attributes permitted on MathML elements in SBML (in addition to the xmlns attribute on math elements):

- style, class and id on any element;

- encoding and definitionURL on csymbol elements; and

- type on cn elements.

Missing values for these attributes are to be treated in the same way as defined by MathML. These restrictions on attributes are designed to confine the MathML elements to their default semantics and to avoid conflicts in the interpretation of the type of token elements.

\subsubsection{Numbers and cn elements}

In MathML, literal numbers are written as the content portion of a particular element called $\mathrm{cn}$. This element takes an optional attribute, type, used to indicate the type of the number (such as whether it is meant to be an integer or a floating-point quantity). Here is an example of its use:

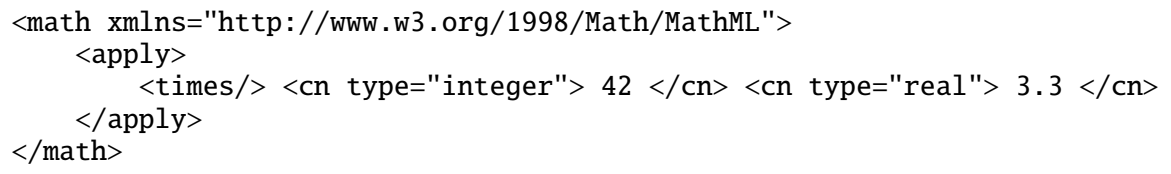


The content of a cn element must be a number. The number can be preceded and succeeded by whitespace (see Section 3.4.5). The following are the only permissible values for the type attribute on MathML cn elements: "e-notation", "real", "integer", and "rational". The value of the type attribute defaults to "real" if it is not specified on a given cn element.

\section{Value space restrictions on cn content}

SBML imposes certain restrictions on the value space of numbers allowed in MathML expressions. According to the MathML 2.0 specification, the values of the content of $\mathrm{cn}$ elements do not necessarily have to conform to any specific floating point or integer representations designed for CPU implementation. For example, in strict MathML, the value of a cn element could exceed the maximum value that can be stored in a IEEE 64 bit floating point number (IEEE 754). This is different from the XML Schema type double that is used in the definition of floating point attributes of objects in SBML; the XML Schema double is restricted to IEEE double-precision 64-bit floating point type IEEE 754-1985. To avoid an inconsistency that would result between numbers elsewhere in SBML and numbers in MathML expressions, SBML Level 2 Version 3 imposes the following restriction on MathML content appearing in SBML:

- Integer values (i.e., the values of $\mathrm{cn}$ elements having type="integer" and both values in $\mathrm{cn}$ elements having type="rational") must conform to the int type used elsewhere in SBML (Section 3.1.3)

- Floating-point values (i.e., the content of $\mathrm{cn}$ elements having type="real" or type="e-notation") must conform to the double type used elsewhere in SBML (Section 3.1.5)

\section{Syntactic differences in the representation of numbers in scientific notation}

It is important to note that MathML uses a style of scientific notation that differs from what is defined in XML Schema, and consequently what is used in SBML attribute values. The MathML 2.0 type "e-notation" (as well as the type "rational") requires the mantissa and exponent to be separated by one $<$ sep/> element. The mantissa must be a real number and the exponent part must be a signed integer. This leads to expressions such as

$<$ cn type="e-notation" $>2<\mathrm{sep} />-5</ \mathrm{cn}>$

for the number $2 \times 10^{-5}$. It is especially important to note that the expression

$<$ cn type="e-notation" $>2 e-5</ c n>$

is not valid in MathML 2.0 and therefore cannot be used in MathML content in SBML. However, elsewhere in SBML, when an attribute value is declared to have the data type double (a type taken from XML Schema), the compact notation "2e-5" is in fact allowed. In other words, within MathML expressions contained in SBML (and only within such MathML expressions), numbers in scientific notation must take the form $<\mathrm{cn}$ type="e-notation" $>2<\mathrm{sep} />-5</ \mathrm{cn}>$, and everywhere else they must take the form "2e-5".

This is a regrettable difference between two standards that SBML replies upon, but it is not feasible to redefine these types within SBML because the result would be incompatible with parser libraries written to conform with the MathML and XML Schema standards. It is also not possible to use XML Schema to define a data type for SBML attribute values permitting the use of the $<$ sep/> notation, because XML attribute values cannot contain XML elements - that is, $<$ sep/> cannot appear in an XML attribute value.

\section{Units of numbers in MathML cn expressions}

What units should be attributed to values appearing inside MathML cn elements? One answer is to assume that the units should be "whatever units appropriate in the context where the number appears". This implies that units can always be assigned unambiguously to any number by inspecting the expression in which it appears, and this turns out to be false. Another answer is that numbers should be considered "dimensionless". Many people argue that this is the correct interpretation, but even if it is, there is an overriding practical reason why it cannot be adopted for SBML's domain of application: when numbers appear in expressions in SBML, they are rarely intended by the modeler to have the unit "dimensionless" 
even if the unit is not declared - the numbers are supposed to have specific units, but the units are usually undeclared. (Being "dimensionless" is not the same as having undeclared units!) If SBML defined numbers as being by default dimensionless, it would result in many models being technically incorrect without the modeler being aware of it unless their software tools performed dimensional analysis. Most software tools today still do not perform dimensional analysis, and so the inconsistency of units (and potential errors in the model) would not be detected until other researchers and database curators attempted to use the model in software packages that did check units. We believe the negative impact on interoperability and people's confidence in SBML as a reliable medium would be too high.

As a result, the current approach in SBML is to leave the default units of literal numbers in MathML content undefined. Software packages and modelers are encouraged to explicitly add unit declarations to numbers. There is a simple mechanism in SBML for associating units with numbers: do not use literal numbers at all; instead, define Parameter objects (Section 4.9) for every quantity, declare units for each such parameter value in its definition, and then insert the parameters in place of numbers in expressions. This leads to mathematical formulas whose units can be fully determined, permitting software tools to perform dimensional analysis and potentially report problems with a model.

In summary: literal numbers appearing within MathML content in SBML have no declared units.

\subsubsection{Use of ci elements in MathML expressions in SBML}

The content of a ci element must be an SBML identifier that is declared elsewhere in the model. The identifier can be preceded and succeeded by whitespace. The set of possible identifiers that can appear in a ci element depends on the containing element in which the ci is used:

- If a ci element appears in the body of a FunctionDefinition object (Section 4.3), the referenced identifier must be either (i) one of the declared arguments to that function, or (ii) the identifier of a previously defined FunctionDefinition object in the model.

- Otherwise, the referenced identifier must be that of a Species, Compartment, Parameter, FunctionDefinition, or Reaction object defined in the model. The following are the only possible interpretations of using such an identifier in SBML:

- Species identifier: When a Species identifier occurs in a ci element, it represents the quantity of that species in units of either amount of substance or units of concentration, depending on the species' definition; see Section 4.8.5.

- Compartment identifier: When a Compartment identifier occurs in a ci element, it represents the size of the compartment. The units of measurement associated with the size of the compartment are those given by the Compartment instance's units attribute value; see Section 4.7.5.

- Parameter identifier: When a Parameter identifier occurs in a ci element, it represents the numerical value assigned to that parameter. The units associated with the parameter's value are those given by the Parameter instance's units attribute; see Section 4.9.3.

- Function identifier: When a FunctionDefinition identifier occurs in a ci element, it represents a call to that function. Function references in MathML occur in the context of using MathML's apply and often involve supplying arguments to the function; see Section 4.3. The units associated with the value returned by the function call are the overall units of the mathematical expression contained in the function definition.

- Reaction identifier: When a Reaction identifier occurs in a ci element, it represents the rate of that reaction as defined by the math expression in the KineticLaw object within the Reaction. The units associated with that rate are substance/time, where the substance and time units established by the values of the SBML built-in units "substance" and "time", respectively. These units may be redefined globally in the model; see Section 4.4.3. If a Reaction instance has no KineticLaw, using its reaction identifier this way is an error (perhaps indicating that the model is incomplete).

The content of ci elements in MathML formulas outside of a KineticLaw or FunctionDefinition must always refer to objects declared in the top level global namespace; i.e., SBML uses "early binding" semantics. Inside 
of KineticLaw, a ci element can additionally refer to local parameters defined within that KineticLaw instance; see Section 4.13.5 for more information.

\subsubsection{Interpretation of boolean values}

As noted already in Section 3.1.2, there is another unfortunate difference between the XML Schema 1.0 and MathML 2.0 standards that impacts mathematical expressions in SBML: in XML Schema, the value space of type boolean includes "true", "false", "1", and " 0 ", whereas in MathML, only "true" and "false" count as boolean values.

The impact of this difference thankfully is minimal because the XML Schema definition is only used for attribute values on SBML objects, and those values turn out never to be accessible from MathML content in SBML - values of boolean attributes on SBML objects can never enter into MathML expressions. Nevertheless, software authors and users should be aware of the difference and in particular that "0" and " 1 " are interpreted as numerical quantities in mathematical expressions. There is no automatic conversion of "0" or "1" to boolean values in contexts where booleans are expected. This allows stricter type checking and unit verification during the validation of mathematical expressions.

\subsubsection{Handling of whitespace}

MathML 2.0 defines "whitespace" in the same way as XML does, i.e., the space character (Unicode hexadecimal code 0020), horizontal tab (code 0009), newline or line feed (code 000A), and carriage return (code 000D). In MathML, the content of elements such as cn and ci can be surrounded by whitespace characters. Prior to using the content, this whitespace is "trimmed" from both ends: all whitespace at the beginning and end of the content is removed (Ausbrooks et al., 2003). For example, in $\langle\mathrm{cn}\rangle 42</ \mathrm{cn}\rangle$, the amount of white space on either side of the " 42 " inside the $<\mathrm{cn}>\ldots</ \mathrm{cn}>$ container does not matter. Prior to interpreting the content, the whitespace is removed altogether.

\subsubsection{Use of csymbol elements in MathML expressions in SBML}

SBML Level 2 uses the MathML csymbol element to denote certain built-in mathematical entities without introducing reserved names into the component identifier namespace. The encoding attribute of csymbol must be set to "text". The definitionURL should be set to one of the following URIs defined by SBML:

- http://www.sbml.org/sbml/symbols/time. This represents the current simulation time. See Section 3.4.7 for more information. The units of the current time entity is determined from the built-in time of Table 2 on page 41.

- http://www.sbml.org/sbml/symbols/delay. This represents a delay function. The delay function has the form delay $(x, d)$, taking two MathML expressions as arguments. Its value is the value of argument $x$ at $d$ time units before the current time. There are no restrictions on the form of $x$. The units of the $d$ parameter are determined from the built-in time. The value of the $d$ parameter, when evaluated, must be numerical (i.e., a number in MathML real, integer, or "e-notation" format) and be greater than or equal to 0 . The delay function is useful for representing biological processes having a delayed response, but where the detail of the processes and delay mechanism is not relevant to the operation of a given model. See Section 3.4 .7 below for additional considerations surrounding the use of this csymbol.

The following examples demonstrate these concepts. The XML fragment below encodes the formula $x+t$, where $t$ stands for time.

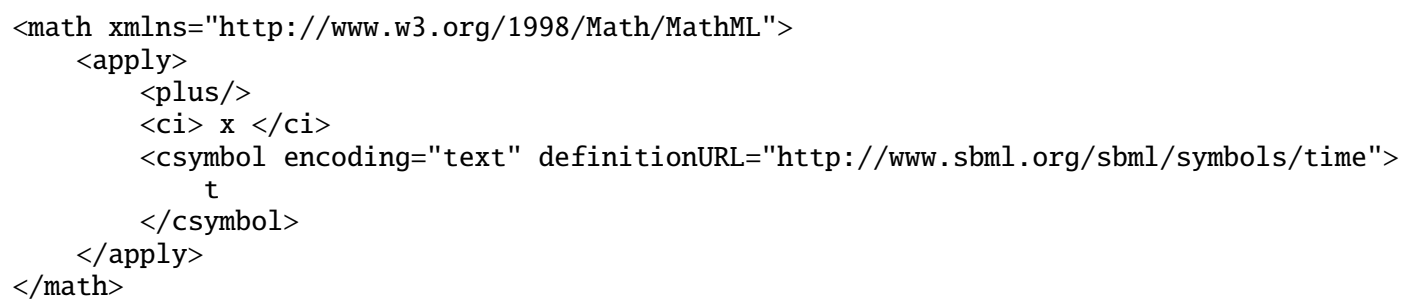


In the fragment above, the use of the token $t$ is mostly a convenience for human readers - the string inside the csymbol could have been almost anything, because it is essentially ignored by MathML parsers and SBML. Some MathML and SBML processors will take note of the token and use it when presenting the mathematical formula to users, but the token used has no impact on the interpretation of the model and it does not enter into the SBML component identifier namespace. In other words, the SBML model cannot refer to $t$ in the example above. The content of the csymbol element is for rendering purposes only and can be ignored by the parser.

As a further example, the following XML fragment encodes the equation $k+\operatorname{delay}(x, 0.1)$ or alternatively $k_{t}+x_{t-0.1}$ :

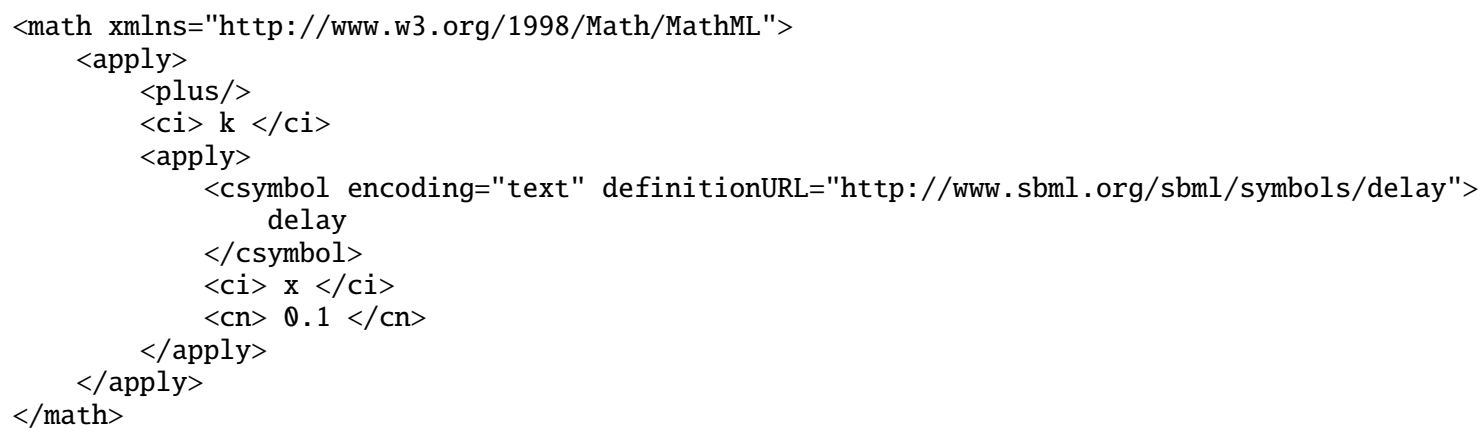

Note that the URI in the value of definitionURL, as all URIs, is intended to serve as a unique identifier and is not intended to be dereferenced as an Internet address. There is nothing actually located at the address http://www.sbml.org/sbml/symbols/delay.

\subsubsection{Simulation time}

The principal use of SBML is to represent quantitative dynamical models whose behaviors manifest themselves over time. In defining an SBML model using constructs such as reactions, time is most often implicit and does not need to be referred to in the mathematical expressions themselves. However, sometimes an explicit time dependency needs to be stated, and for this purpose, the time csymbol (described above in Section 3.4.6) may be used. This time symbol refers to "instantaneous current time" in a simulation, frequently given the literal name $t$ in one's equations.

An assumption in SBML is that "start time" or "initial time" in a simulation is zero, that is, if $t_{0}$ is the initial time in the system, $t_{0}=0$. This corresponds to the most common scenario. Initial conditions in SBML take effect at time $t=0$. There is no mechanism in SBML for setting the initial time to a value other than 0 . To refer to a different time in a model, one approach is to define a Parameter for a new time variable and use an AssignmentRule in which the assignment expression subtracts a value from the csymbol time. For example, if the desired offset is 2 time units, the MathML expression would be

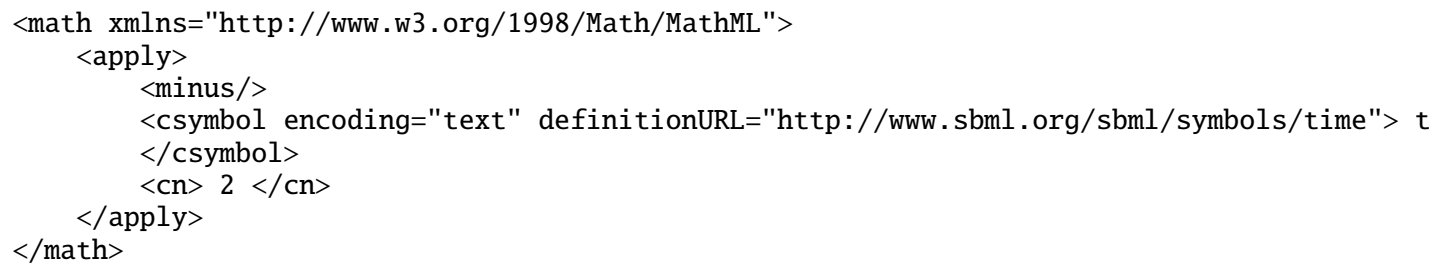

SBML's assignment rules (Section 4.11.3) can be used to express mathematical statements that hold true at all moments, so using an assignment rule with the expression above will result in the value being equal to $t-2$ at every point in time. A parameter assigned this value could then be used elsewhere in the model, its value could be plotted by a simulator, etc. 


\subsubsection{Initial conditions and special considerations}

The identifiers of Species, Compartment, Parameter, and Reaction object instances in a given SBML model refer to the main variables in a model. Depending on certain attributes of these objects (e.g., the attribute constant on species, compartments and parameters - this and other conditions are explained in the relevant sections elsewhere in this document), some of the variables may have constant values throughout a simulation, and others' values may change. These changes in values over time are determined by the system of equations constructed from the model's reactions, initial assignments, rules, and events.

As described in Section 3.4.7, an SBML model's simulation is assumed to begin at $t=0$. The availability of the delay csymbol (Section 3.4.6) introduces the possibility that at $t \geq 0$, mathematical expressions in a model may draw on values of model components from time prior to $t=0$. A simulator may therefore need to compute the values of variables at time points $t_{i} \leq 0$ to allow the calculation of values required for the evaluation of delay expressions in the model for $t \geq 0$. If there are no delays in the model, then $t_{i}=0$.

The following is how the definitions of the model should be applied:

1. At time $t_{i}$ :

- Every Species, Compartment, and Parameter whose definition includes an initial value is assigned that value. If an element has constant= "false", its value may be changed by other constructs or reactions in a model according to the steps below; if constant="true", only an InitialAssignment can override the value.

- All InitialAssignment definitions take effect at $t_{i}$ and continue to have effect up to and including $t=$ 0 , overriding any initial values on Species, Compartment and Parameter. Since InitialAssignments contain mathematical formulas, different values may be computed at each time step $t$ in $t_{i} \leq t \leq 0$.

2. For time $t \geq t_{i}$ :

- AssignmentRule and AlgebraicRule definitions are in effect from this point in time forward and may influence the values of Species quantity, Compartment size, and Parameter values. (Note there cannot be both an AssignmentRule and an InitialAssignment for the same identifier; see Section 4.11.)

3. At time $t=0$ :

- The system of equations constructed by combining AssignmentRule equations, AlgebraicRule equations, RateRule equations, and the equations constructed from the Reaction definitions in the model, are used to obtain consistent initial conditions for numerical solver algorithms. (Note that there cannot be both an AssignmentRule and a RateRule for the same identifier, or both an AssignmentRule and an InitialAssignment for the same identifier; see Section 4.11.3.)

- Constraint definitions begin to take effect (and a constraint violation may result; see Section 4.12).

4. For time $t>0$ :

- RateRule definitions can begin to take effect.

- Event definitions can begin to take effect. (Note that an Event cannot be defined to change the value of a variable that is also the subject of an AssignmentRule; see Section 4.14.)

- System simulation proceeds.

To reiterate: in modeling situations that do not involve the use of the delay csymbol, $t_{i}=0$, but this does not alter the steps above.

\subsubsection{MathML expression data types}

MathML operators in SBML each return results in one of two possible types: boolean and numerical. By numerical type, we mean either (1) a number in MathML real, integer, rational, or "e-notation" format; or (2) the csymbol for delay or the csymbol for time described in Section 3.4.6. The following guidelines summarize the different possible cases. 
The relational operators (eq, neq, gt, lt, geq, leq), the logical operators (and, or, xor, not), and the boolean constants (false, true) always return boolean values. As noted in Section 3.4.4, the numbers $\mathbb{0}$ and 1 do not count as boolean values in MathML contexts in SBML.

The type of an operator referring to a FunctionDefinition is determined by the type of the top-level operator of the expression in the math element of the FunctionDefinition instance, and can be boolean or numerical.

All other operators, values and symbols return numerical results.

The roots of the expression trees used in the following contexts must yield boolean values:

- the arguments of the MathML logical operators (and, or, xor, not);

- the second argument of a MathML piece operator;

- the trigger element of an SBML Event; and

- the math element of an SBML Constraint.

The roots of the expression trees used in the following contexts can optionally yield boolean values:

- the arguments to the eq and neq operators;

- the first arguments of MathML piece and otherwise operators; and

- the top level expression of a function definition.

The roots of expression trees in other contexts must yield numerical values.

The type of expressions should be used consistently. The set of expressions that make up the first arguments of the piece and otherwise operators within the same piecewise operator should all return values of the same type. The arguments of the eq and neq operators should return the same type.

\subsubsection{Consistency of units in mathematical expressions and treatment of unspecified units}

Strictly speaking, physical validity of mathematical formulas requires not only that physical quantities added to or equated with each other have the same fundamental dimensions and units of measurement; it also requires that the application of operators and functions to quantities produces sensible results. Yet, in real-life models today, these conditions are often and sometimes legitimately disobeyed. SBML must again balance the ability of modelers to represent whatever model they desire against the need for the represented models to be self-consistent and detailed enough for different software tools to be able to manipulate and analyze them.

The approach taken in SBML is to focus requirements principally on preventing inconsistency of a model. In a nutshell, the SBML requirement is that what is expressed in a model must be consistent to the extent that this can be determined using the information available in the model.

\section{Unit requirements for mathematical expressions}

The SBML requirement for consistency of units is defined in terms of dimensional analysis applied recursively to every operator and function and every argument to them. Some conditions must be true, and others should be true (but are not absolutely required for model validity). The following conditions must be true in a model:

1. All arguments to the following operators must have the same units (regardless of what those units happen to be): plus, minus, eq, neq gt, lt, geq, leq.

2. The units of each argument in a call to a FunctionDefinition must match the units expected by the lambda expression within the math expression of that FunctionDefinition instance.

3. All of the possible return values from piece and otherwise subelements of a piecewise expression must have the same units, regardless of what those units are. (Otherwise, the piecewise expression would return values having different units depending on which case evaluated to true.)

4. For the delay csymbol (Section 3.4.6) function, which has the form delay $(x, d)$, the second argument $d$ must match the model's unit of time (i.e., the "time" built-in unit). 
In addition to the above, the following conditions should hold true (and software developers may wish to consider having their software warn users if one or more of the following conditions is not true):

1. The units of each argument to the following operators should be "dimensionless": exp, ln, log, factorial, sin, cos, tan, sec, csc, cot, sinh, cosh, tanh, sech, csch, coth, arcsin, arccos, arctan, arcsec, arccsc, arccot, arcsinh, arccosh, arctanh, arcsech, arccsch, arccoth.

2. The two arguments to power, which are of the form power $(a, b)$ with the meaning $a^{b}$, should be as follows: (1) if the second argument is an integer, then the first argument can have any units; (2) if the second argument $b$ is a rational number $n / m$, it must be possible to derive the $m$-th root of $(a\{\text { units }\})^{n}$, where $\{$ units $\}$ signifies the units associated with $a$; otherwise, (3) the units of the first argument must be "dimensionless". The second argument $(b)$ should always have units of "dimensionless".

3. The two arguments to root, which are of the form $\operatorname{root}(n, a)$ with the meaning $\sqrt[n]{a}$ and where the degree $n$ is optional (defaulting to "2"), should be as follows: (1) if the optional degree qualifier $n$ is an integer, then it must be possible to derive the $n$-th root of $a$; (2) if the optional degree qualifier $n$ is a rational $n / m$ then it must be possible to derive the $n$-th root of $(a\{\text { units }\})^{m}$, where $\{$ units $\}$ signifies the units associated with $a$; otherwise, (3) the units of $a$ must be "dimensionless".

4. Since the units of literal numbers cannot be specified directly in SBML (see below), it is possible for the units of a FunctionDefinition object's return value to be effectively different in different contexts where it is called. If a FunctionDefinition's mathematical formula contains literal constants (i.e., numbers within MathML cn elements), the units of the constants should be identical in all contexts the function is called.

The units of other operators such as abs, floor, and ceiling, can be anything.

The final bulleted item above, regarding FunctionDefinition, warrants additional elaboration. An example may help illustrate the problem. Suppose the formula $x+5$ is defined as a function, where $x$ is an argument. The literal number 5 in SBML has unspecified units. If this function is called with an argument in moles, the only possible consistent unit for the return value is mole. If in another context in the same model, the function is called with an argument in seconds, the function return value can only be treated as being in seconds. Now suppose that a modeler decides to change all uses of seconds to milliseconds in the model. To make the function definition return the same quantity in terms of seconds, the 5 in the formula would need to be changed, but doing so would change the result of the function everywhere it is called - with the wrong consequences in the context where moles were intended. This illustrates the subtle danger of using numbers with unspecified units in function definitions. There are at least two approaches for avoiding this: (1) define separate functions for each case where the units of the constants are supposed to be different; or (2) declare the necessary constants as Parameter objects in the model (with declared units!) and pass those parameters as arguments to the function, avoiding the use of literal numbers in the function's formula.

\section{Treatment of unspecified units}

There are only two ways to introduce numbers with unspecified units into mathematical formulas in SBML: using literal numbers (i.e., numbers enclosed in MathML cn elements), and using Parameter objects defined without unit declarations. All other quantities, in particular species and compartments, always have unit declarations (whether explicit or the defaults).

If an expression contains literal numbers and/or Parameter objects without declared units, the consistency or inconsistency of units may be impossible to determine. In the absence of a verifiable inconsistency, an expression in SBML is accepted as-is; the writer of the model is assumed to have written what they intended. Nevertheless, this is not equivalent to assuming the expression does have consistent units.

In some cases, it may be possible to determine that expressions containing unspecified units are inconsistent regardless of what units would be attributed to the unspecified quantities. For example, the expression

$$
\frac{d X}{d t}=\frac{[Y] \cdot[Z]^{n}}{[Z]^{m}+1} \cdot V
$$


with $X, Y$ and $Z$ in units of substance, $V$ in units of volume, and $m \neq n$, cannot ever be consistent, no matter what units the literal 1 takes on. (This also illustrates the need not to stop verifying the units of an expression immediately upon encountering an unspecified quantity - the rest of the expression may still be profitably evaluated and checked for inconsistency.)

We advise modelers and software tools to declare the units of all quantities in a model, insofar as this is possible, using the various mechanisms provided for this in SBML. Fully declared units can allow software tools to perform dimensional analysis on the units of mathematical expressions, and such analysis can be valuable in helping modelers produce correct models. In addition, it can allow model-wide operations such as conversion or rescaling of units. The lack of declared units on quantities in a model does not render it invalid SBML, but it reduces the types of consistency checks and useful operations (such as conversions and translations) that software systems can perform.

\subsubsection{SBML does not define implicit unit conversions}

Implicit unit conversions do not exist in SBML. Consider the following example. Suppose that in some model, a species $S_{1}$ has been declared as having a mass of $1 \mathrm{~kg}$, and a second species $S_{2}$ has been declared as having a mass of $500 \mathrm{~g}$. What should be the result of evaluating an expression such as $S_{1}>S_{2}$ ? If the numbers alone are considered,

$$
1>500
$$

would evaluate to "false", but if the units were implicitly converted by the software tool interpreting the model,

$$
1 k g>500 g
$$

would evaluate to "true". This is a trivial example, but the problem for SBML is that implicit unit conversions of this kind can lead to controversial situations where even humans do not agree on the answer. Consequently, SBML only requires that mathematical expressions be evaluated numerically. It is up to the model writer to ensure that the units on both sides of an expression match, by inserting explicit unit conversion factors if necessary.

It is simple enough to avoid expressions with mixed units such as in the example above: a modeler or a software tool can define a parameter that acts as a conversion factor, and then multiply one of the terms by this parameter. Thus, a model could include a parameter " $g_{-} p e r_{-} k g$ " with a value of 1000 , and the expression could be written as

$$
1 * g \_p e r \_k g>500
$$

which will then evaluate to "true". 


\section{SBML components}

In this section, we define each of the major components of SBML. We use the UML notation described in Section 1.4.3 for defining classes of objects. We also illustrate the use of SBML components by giving partial model definitions in XML. Section 7 provides many full examples of SBML in XML.

\subsection{The SBML container}

All well-formed XML documents must begin with an XML declaration, which specifies both the version of XML assumed and the document character encoding. The declaration begins with the characters $<$ ? xml followed by the XML version and encoding attributes. SBML Level 2 uses XML version 1.0 and requires a document encoding of UTF-8. Following this XML declaration, the outermost portion of a model expressed in SBML Level 2 Version 3 consists of an object of class Sbml, defined in Figure 9. This class contains two required attributes, for the SBML level and version, and one required subelement called model whose class is Model.

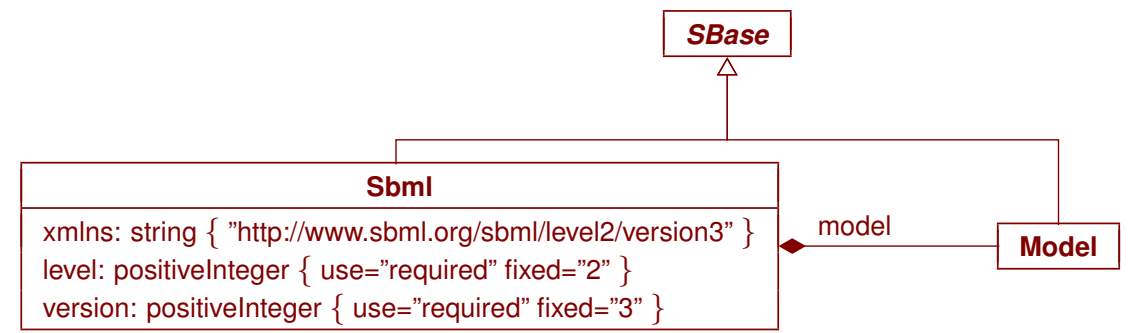

Figure 9: The definition of Sbml for SBML Level 2 Version 3. The class Model is defined in Section 4.2. Note that both $\mathrm{Sbml}$ and Model are subclasses of Sbase, and therefore inherit the attributes of that abstract class.

The following is an abbreviated example of these XML elements for an SBML Level 2 Version 3 document:

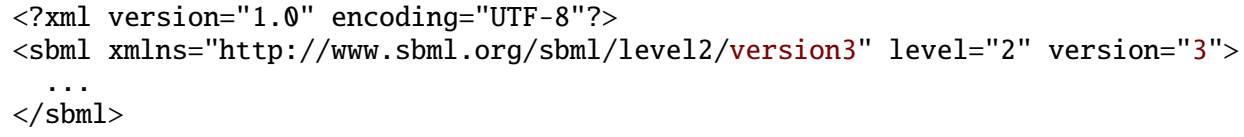

The attribute xmlns declares the default XML namespace used within the sbml element. The URI for SBML Level 2 Version 3 is http://www.sbml.org/sbml/level2/version3. All elements must be placed in this namespace either by assigning the default namespace as shown above, or using a tag prefix on every element.

An SBML XML document must not contain elements or attributes in the SBML namespace that are not defined in this SBML Level 2 Version 3 Release 1 specification. Documents containing unknown elements or attributes placed in the SBML namespace do not conform to this SBML specification.

Readers may wonder why the SBML top-level XML element uses both a namespace URI identifying the SBML level and version, as well as separate XML attributes giving the level and version. Why is the information duplicated? There are several reasons. First, XML is only one possible serialization of SBML (albeit an extremely popular one at this time). Though most of this document is written with XML in mind, it is the intention behind the design of SBML that its object structure should be implementable in other languages and software systems. Programmatic access is easier if the level and version information are accessible directly as data rather than have to be extracted from a string. Second, generic high-level XML parsers may not give their calling programs access to the value of the xmlns attribute. Providing the information via separate attributes is a good backup measure. And finally, earlier in the history of SBML, it was expected that only the level needed to be encoded as part of the namespace URI (e.g., http://www.sbml.org/sbml/level1) because it was hoped that changes within levels would not require XML Schema changes. This has proven to be false, but SBML Level 1 (both versions) and the first version of SBML Level 2 still subscribe to this principle. This means that for these variants of SBML, software tools must look for a version attribute on the top-level element. For backwards compatibility with software that expects this, it makes more sense to keep the version and level attributes. 


\subsection{Model}

The definition of Model is shown in Figure 10 on the following page. Only one instance of a Model object is allowed per instance of an SBML Level 2 Version 3 Release 1 document or data stream, and it must be located inside the $<\operatorname{sbml}>\ldots</$ sbml $>$ element as described in Section 4.1.

The Model object has an optional attribute, id, used to give the model an identifier. The identifier must be a text string conforming to the syntax permitted by the SId data type described in Section 3.1.7. Model also has an optional name attribute, of type string. The name and id attributes must be used as described in Section 3.3.

Model serves as a container for components of classes FunctionDefinition, UnitDefinition, CompartmentType, SpeciesType, Compartment, Species, Parameter, InitialAssignment, Rule, Constraint, Reaction and Event. Instances of the classes are placed inside instances of classes ListOfFunctionDefinitions, ListOfUnitDefinitions, ListOfCompartmentTypes, ListOfSpeciesTypes, ListOfCompartments, ListOfSpecies, ListOfParameters, ListOfInitialAssignments, ListOfRules, ListOfConstraints, ListOfReactions, and ListOfEvents. The "list" classes are defined in Figure 10. All of the lists are optional, but if a given list container is present within the model, the list must not be empty; that is, it must have length one or more. The resulting XML data object for a full model containing every possible list would have the following form:

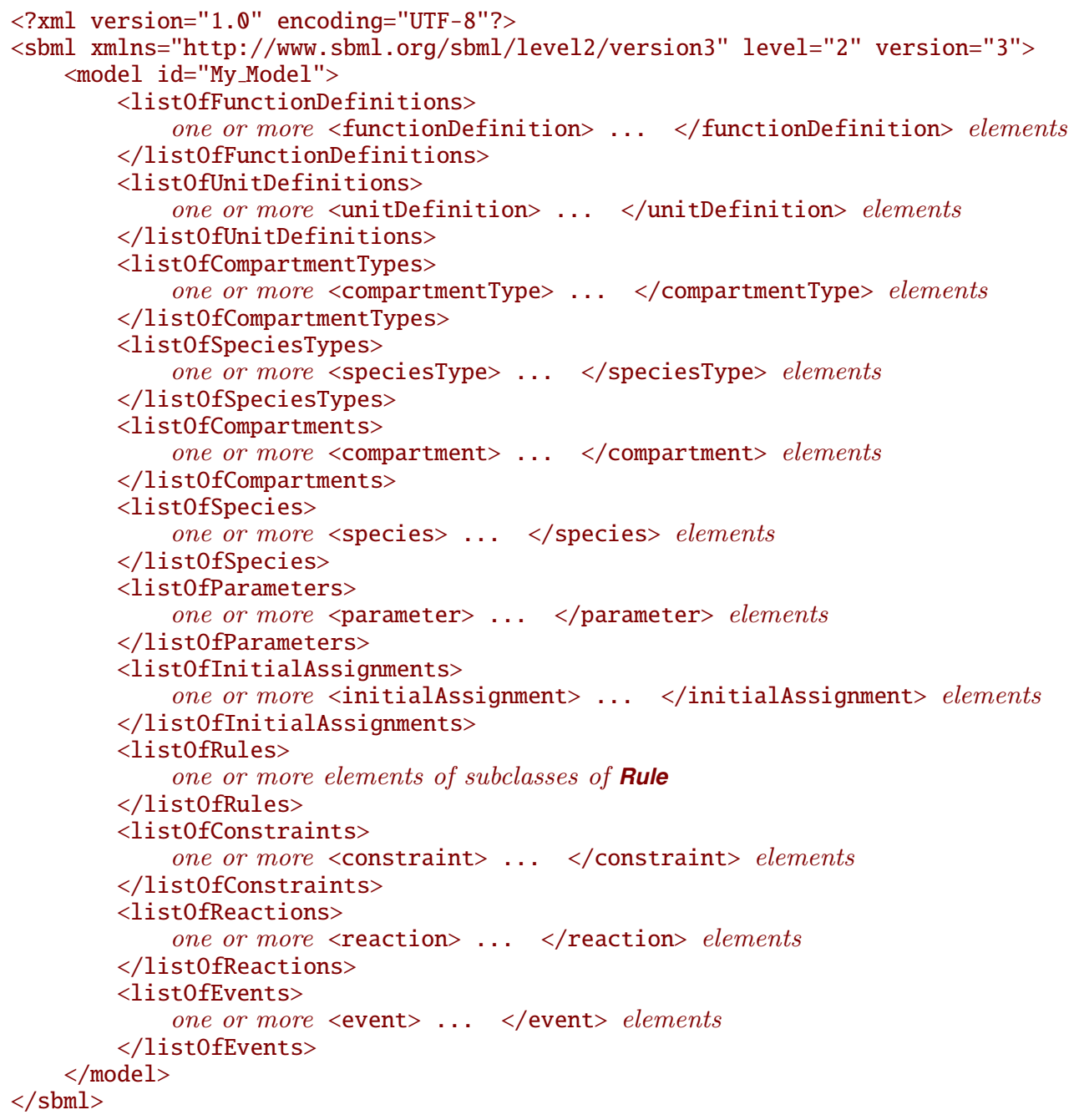

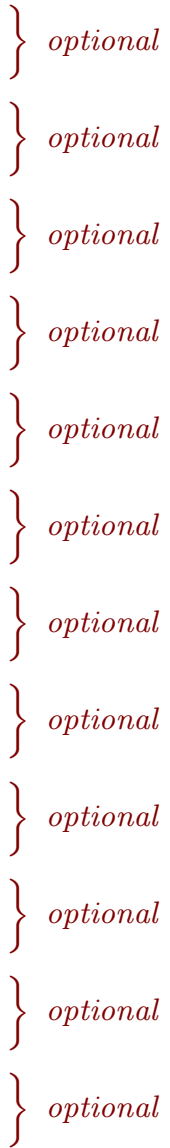

Although all the lists are optional, there are dependencies between SBML components such that defining some components requires defining others. An example is that defining a species requires defining a compartment, and defining a reaction requires defining a species. The dependencies are explained throughout the text. 


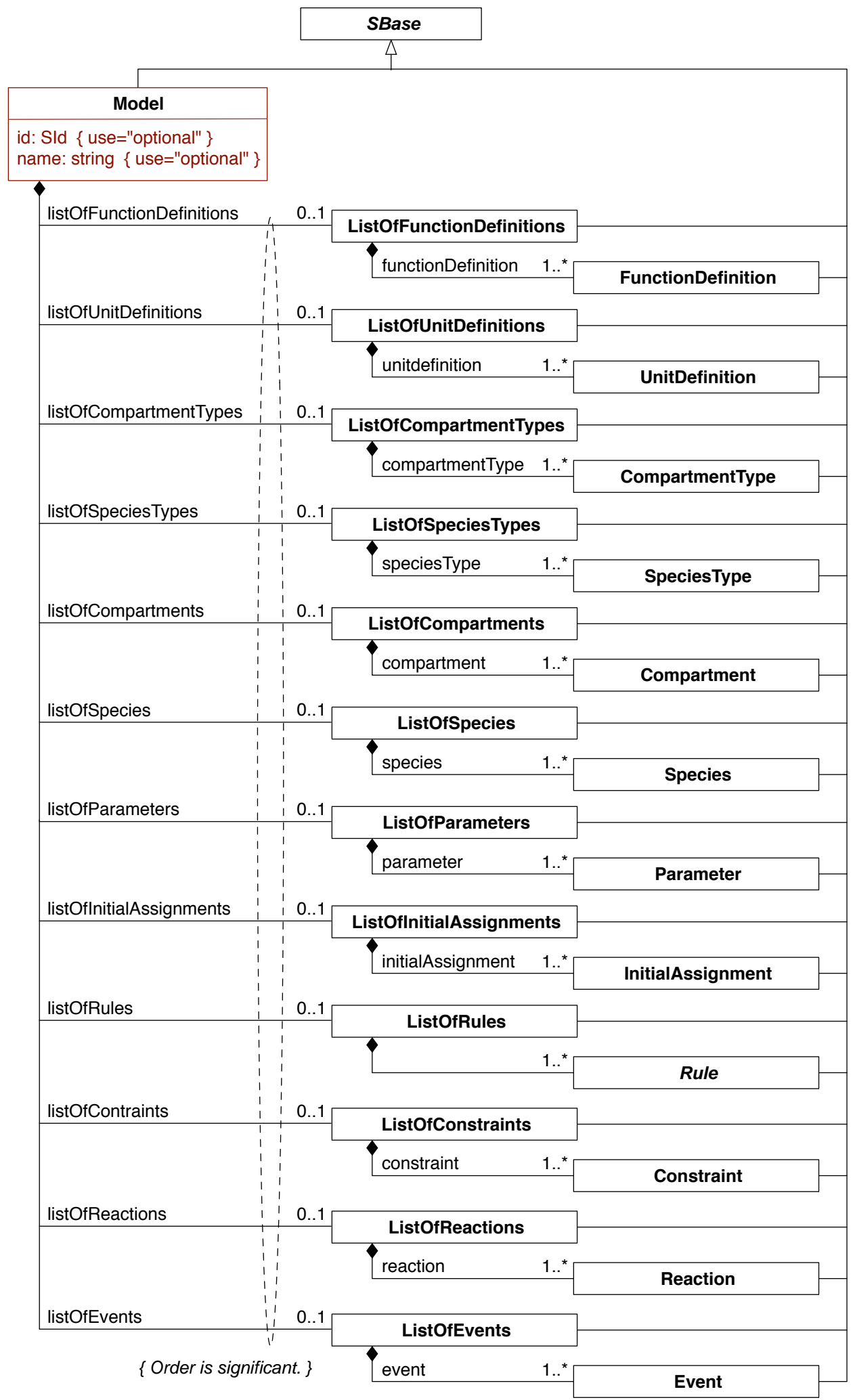

Figure 10: The definition of Model and the many helper classes ListOfFunctionDefinitions, ListOfUnitDefinitions, ListOfCompartmentTypes, ListOfSpeciesTypes, ListOfCompartments, ListOfSpecies, ListOfParameters, ListOfInitialAssignments, ListOfRules, ListOfConstraints, ListOfReactions, and ListOfEvents. 


\subsubsection{The ListOf container classes}

The various Listof__ classes defined in Figure 10 are merely containers used for organizing the main components of an SBML model. All are derived from the abstract class Sbase (Section 3.2), and inherit Sbase's various attributes and subelements such as metaid and annotation, although in SBML Level 2 Version 3 Release 1 there are no defined SBO terms for the sboTerm attribute. The Listof__ classes do not add any attributes of their own.

Readers may wonder about the motivations for using the Listof__ containers. A simpler approach in XML might be to place the components all directly at the top level. We chose instead to group them within XML elements named after list0fClassNames, in part because we believe this helps organize the components and makes visual reading of models in XML easier. More importantly, the fact that the container classes are derived from Sbase means that software tools can add information about the lists themselves into each list container's annotation, a feature that a number of today's software tools exploit.

\subsubsection{The sboTerm attribute}

The Model object has an optional sboTerm attribute of type SBOTerm (see Sections 3.1.9 and 5). Values of this attribute must be chosen from identifiers referring to a modeling framework defined in SBO (i.e., terms derived from SB0:0000004, "modeling framework"). The SBO term chosen should be the most precise (narrow) term that defines the mathematical framework assumed by the entire model. An example might be "non-spatial discrete framework", which would mean the entire model was created under the assumption it will be simulated in a tool that turns substance quantities into discrete numbers and applies a stochastic simulation algorithm (e.g., Gillespie, 1977) to the model. Other frameworks are possible.

A dependency exists between the SBO term referenced by a Model instance and the SBO labels on individual KineticLaw instances within the model. In SBO, each definition of a rate expression itself includes an SBO reference to a mathematical framework. When a given KineticLaw element refers to a rate law term in SBO, it therefore simultaneously also identifies a framework. (It is possible for a model to mix frameworks, which is why it is important to enable individual reactions to indicate which one is assumed.) By contrast, if all the reactions in a given model use the same framework, it makes sense to characterize the model as a whole as assuming this. Thus, the label on Model should apply to the model as a whole, meaning that if Model's sboTerm attribute has a value, all of that model's reaction rate expressions are assumed to be expressed using the same mathematical framework, and the application reading the model may ignore frameworks indicated by the definitions of the SBO terms referenced by the KineticLaw elements's sboTerm attributes. As discussed in Section 5, SBO labels are optional information on a model.

The absence of an sboTerm value on a Model instance means that either the model has not been labeled with SBO terms at all, or no modeling framework term in SBO at the time the model was created was deemed suitable for characterizing the model as a whole. If a Model instance has no value for its sboTerm attribute, the definitions of the SBO terms referenced by individual KineticLaw elements in the model may indicate the framework assumed for each reaction separately.

\subsection{Function definitions}

The FunctionDefinition object associates an identifier with a function definition. This identifier can then be used as the function called in subsequent MathML apply elements. FunctionDefinition is shown in Figure 11 on the next page.

Function definitions in SBML (also informally known as "user-defined functions") have purposefully limited capabilities. As is made more clear below, a function cannot reference parameters or other model quantities outside of itself; values must be passed as parameters to the function. Moreover, recursive and mutuallyrecursive functions are not permitted. The purpose of these limitations is to balance power against complexity of implementation. With the restrictions as they are, function definitions could be implemented as textual substitutions - they are simply macros. Software implementations therefore do not need the full functiondefinition machinery typically associated with programming languages. 


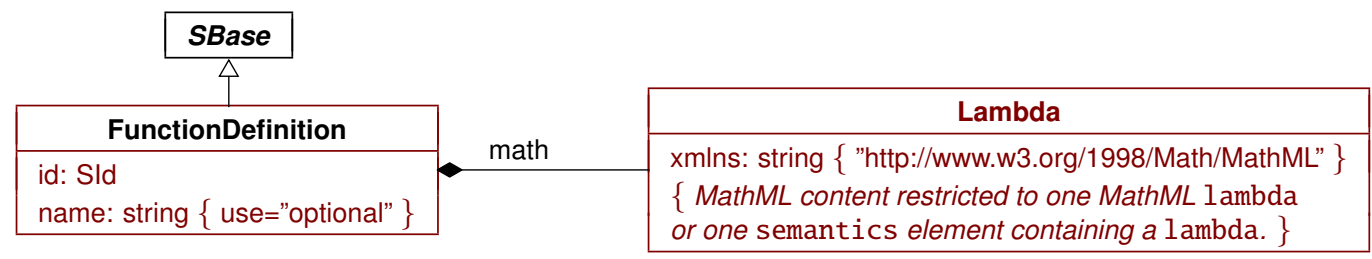

Figure 11: The definition of class FunctionDefinition. The contents of the Lambda class is a single MathML lambda expression (or a lambda surrounded by a semantics element). A function definition must contain exactly one math element defined by the Lambda class; note also that Lambda is not derived from Sbase, which means that the attributes defined on Sbase are not available on the math element. A sequence of one or more instances of FunctionDefinition objects can be located in an instance of ListOfFunctionDefinitions in Model, as shown in Figure 10.

\subsubsection{The id and name attributes}

The id and name attributes have types SId and string, respectively, and operate in the manner described in Section 3.3. MathML ci elements in an SBML model can refer to the function defined by a FunctionDefinition using the value of its id attribute.

\subsubsection{The math element}

The math element is a container for MathML content that defines the function. The content of this element can only be a MathML lambda element or a MathML semantics element containing a lambda element. The lambda element must begin with zero or more bvar elements, followed by any other of the elements in the MathML subset listed in Section 3.4.1 except lambda (i.e., a lambda element cannot contain another lambda element). This is the only place in SBML where a lambda element can be used. The function defined by a FunctionDefinition is only available for use in other MathML elements that follow the FunctionDefinition definition in the model. (These restrictions prevent definition of recursive and mutually-recursive functions.)

A further restriction on the content of math is that it cannot contain references to variables other than the variables declared to the lambda itself. That is, the contents of MathML ci elements inside the body of the lambda can only be the variables declared by its bvar elements, or the identifiers of other FunctionDefinitions defined earlier in the model. This restriction also applies to the csymbol for time. Functions must be written so that all variables or parameters used in the MathML content are passed to them via their function parameters.

Note that FunctionDefinition does not have a separate attribute for defining the units of the value returned by the function. The units associated with the function's return value, when the function is called from within MathML expressions elsewhere in SBML, are simply the overall units of the expression in FunctionDefinition's math when applied to the arguments supplied in the call to the function. Ascertaining these units requires performing dimensional analysis on the expression. (Readers may wonder why there is no attribute. The reason is that having a separate attribute for declaring the units would not only be redundant, but also lead to the potential for having conflicting information. In the case of a conflict between the declared units and those of the value actually returned by the function, the only logical resolution rule would be to assume that the correct units are those of the expression anyway.)

\subsubsection{The sboTerm attribute}

FunctionDefinition inherits an optional sboTerm attribute of type SBOTerm from its parent class Sbase (see Sections 3.1.9 and 5). When a value is given to this attribute in a FunctionDefinition instance, it must be an SBO identifier referring to a mathematical expression (i.e., terms derived from SB0:0000064, "mathematical expression"). The relationship is of the form "the function definition is a X", where X is the SBO term. The term chosen should be the most precise (narrow) one that captures the role of the function in the model.

As discussed in Section 5, SBO labels are optional information on a model. Applications are free to ignore sboTerm values. A model must be interpretable without the benefit of SBO labels. 


\subsubsection{Examples}

The following abbreviated SBML example shows a FunctionDefinition object instance defining pow3 as the identifier of a function computing the mathematical expression $x^{3}$, and after that, the invocation of that function in the mathematical formula of a rate law. Note how the invocation of the function uses its identifier.

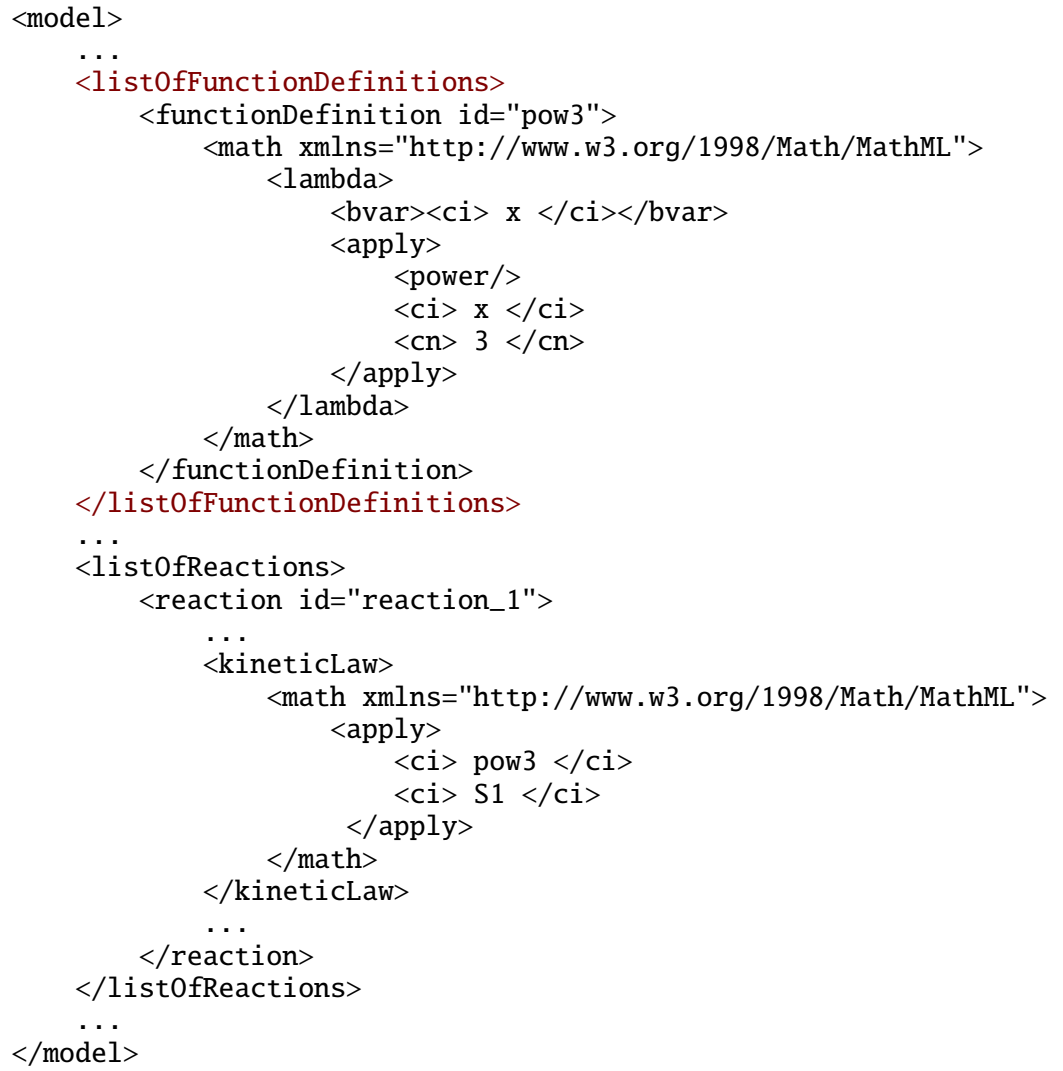

\subsection{Unit definitions}

Units of measurement may be supplied in a number of contexts in an SBML model. The units of the following mathematical entities can be specified explicitly: the size of a Compartment, the initial amount of a Species, and the units of constant and variable Parameter values. The overall units of any mathematical formula appearing in SBML are those that arise naturally from the components and mathematical expressions comprising the formula, or in other words, the units obtained by doing dimensional analysis on the formula.

Rather than requiring a complete unit definition on every object, SBML provides a facility for defining units that can be referenced throughout a model. In addition, every kind of SBML mathematical entity has units assigned to it from a set of built-in defaults (see Section 4.4.3 below, and also Sections 4.7.5, 4.8.5 and 4.13.5). By redefining these built-in default units, it is possible to change the units used throughout a model in a simple and consistent manner. The SBML unit definition facility uses two classes of objects, UnitDefinition and Unit. Their definitions are shown in Figure 12 on the following page and explained in more detail in Sections 4.4.1 and 4.4.2 below.

The approach to defining units in SBML is compositional; for example, meter second ${ }^{-2}$ is constructed by combining a Unit object representing meter with another Unit object representing second ${ }^{-2}$. The combination is wrapped inside a UnitDefinition, which provides for assigning an identifier and optional name to the combination. The identifier can then be referenced from elsewhere in a model.

The vast majority of modeling situations requiring new SBML unit definitions involve simple multiplicative combinations of base units and factors. An example of this might be "moles per litre per second". What distinguishes these sorts of simpler unit definitions from more complex ones is that they may be expressed 


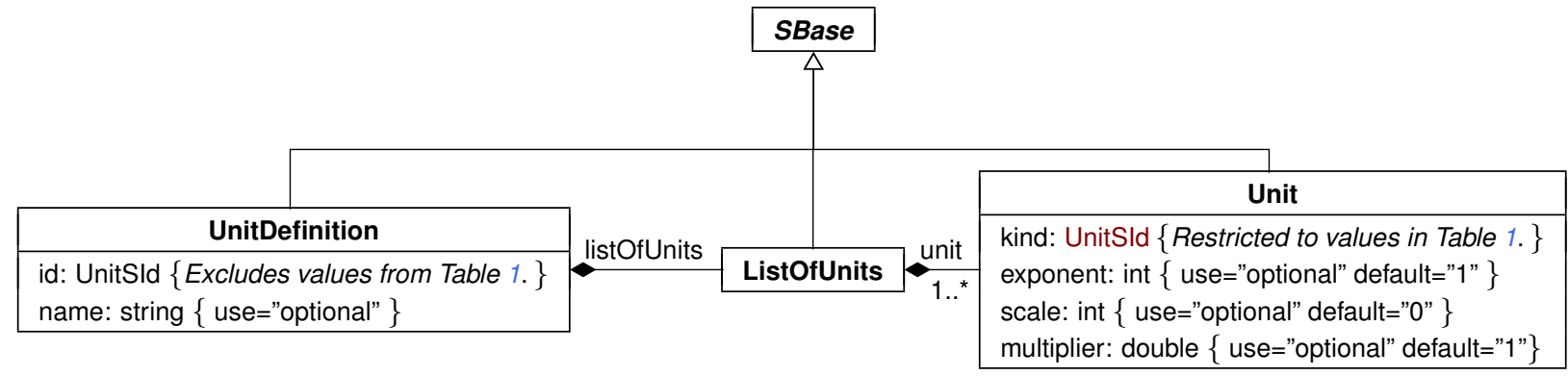

Figure 12: The definition of classes UnitDefinition and Unit. A sequence of one or more instances of UnitDefinition can be located in an instance of ListOfUnitDefinitions in Model (Figure 10). ListOfUnits has no attributes (beyond those it inherits from class Sbase); it merely acts as a container for one or more instances of Unit objects. Note that the only permitted values of kind on Unit are the reserved words in Table 1 on the following page, but these symbols are excluded from the permitted values of UnitDefinition's id because SBML's unit system does not allow redefining the base units.

without the use of an additive offset from a zero point. The use of offsets complicates all unit definition systems, yet in the domain of SBML the real-life cases requiring offsets are few (and in fact, to the best of our knowledge, only involve temperature). Consequently, the SBML unit system has been consciously designed in a way that attempts to simplify implementation of unit support for the most common cases in systems biology, at the cost of requiring units with offsets to be handled explicitly by the modeler.

\subsubsection{UnitDefinition}

A unit definition in SBML consists of an instance of a UnitDefinition object, shown in Figure 12.

\section{The id and name attributes}

The required attribute id and optional attribute name have data types UnitSId and string, respectively. The id attribute is used to give the defined unit a unique identifier by which other parts of an SBML model definition can refer to it. The name attribute is intended to be used for giving the unit definition an optional human-readable name; see Section 3.3.2 for more guidelines about the use of names.

There are two important restrictions and guidelines about the use of unit definition id values:

1. The id of a UnitDefinition must not contain a value from Table 1, the list of reserved base unit names. This constraint simply prevents the redefinition of base units.

2. There is a set of predefined identifiers for the built-in default units in SBML; these identifiers are "substance", "volume", "area", "length", and "time". Using one of these values for id in a UnitDefinition has the effect of redefining the model-wide default units for the corresponding quantities. We discuss this in more detail in Section 4.4.3.

\section{The list of Units}

A UnitDefinition object must contain one or more Unit objects inside a ListOfUnits container. Section 4.4.2 explains the meaning and use of Unit.

\section{Example}

The following skeleton of a unit definition illustrates an example use of UnitDefinition:

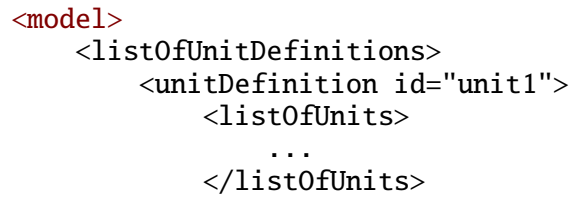




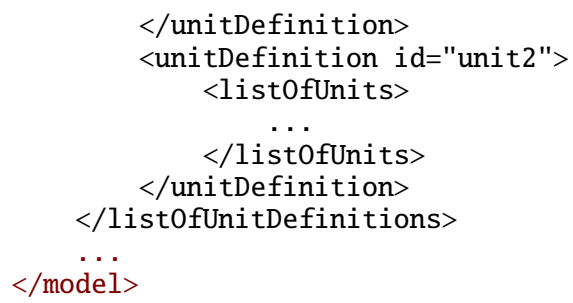

\subsubsection{Unit}

A Unit object represents a (possibly transformed) reference to a base unit chosen from the list in Table 1. The attribute kind indicates the chosen base unit, whereas the attributes exponent, scale, and multiplier define how the base unit is being transformed. These various attributes are described in detail below.

In SBML Level 2 Version 1, Unit had an additional attribute called offset. This attribute has been removed entirely in Level 2 Versions 2 and 3. Modelers and software authors are instead directed to use other methods of encoding units requiring offsets. The reasons for this change, and some suggestions for how to achieve equivalent effects of unit offsets, are discussed in more detail below. Another change in Version 3 is the removal of the enumeration UnitKind and the redefinition of UnitSId to include the previous UnitKind values as reserved symbols. This change has no net effect on permissible models or their representation.

\section{The kind attribute}

The Unit object class has one required attribute, kind, whose value must be taken from the list of reserved words given in Table 1. These reserved symbols are in the value space of UnitSId (Section 3.1.8).

\begin{tabular}{llllll}
\hline ampere & gram & katal & metre & second & watt \\
becquerel & gray & kelvin & mole & siemens & weber \\
candela & henry & kilogram & newton & sievert & \\
coulomb & hertz & litre & ohm & steradian & \\
dimensionless & item & lumen & pascal & tesla & \\
farad & joule & lux & radian & volt & \\
\hline
\end{tabular}

Table 1: Base units defined in SBML. These symbols are predefined values of type UnitSId (Section 3.1.8). All are names of base or derived SI units (Bureau International des Poids et Mesures, 2000), except for "dimensionless" and "item", which are SBML additions important for handling certain common situations. "Dimensionless" is intended for cases where a quantity is a ratio whose units cancel out, and " $\mathrm{i}$ tem" for expressing such things as "N items" (e.g., "100 molecules"). Also, note that the gram and litre are not strictly part of SI; however, they are frequently used in SBML's areas of application and therefore are included as predefined unit identifiers. (The standard SI unit of mass is in fact the kilogram, and volume is defined in terms of cubic metres.) Comparisons of these values, like all values of type UnitSId, must performed in a case-sensitive manner.

Note that the set of acceptable values for the attribute kind does not include units defined by UnitDefinition object. This means that the units definition system in SBML is not hierarchical: user-defined units cannot be built on top of other user-defined units, only on top of base units. SBML differs from CellML (Hedley et al., 2001) in this respect; CellML allows the construction of hierarchical unit definitions.

\section{The exponent, scale and multiplier attributes}

The optional exponent attribute on Unit represents an exponent on the unit. Its default value is "1" (one). A Unit object also has an optional scale attribute; its value must be an integer exponent for a power-often multiplier used to set the scale of the unit. For example, a unit having a kind value of "gram" and a scale value of "-3" signifies $10^{-3} \times$ gram, or milligrams. The default value of scale is " 0 " (zero), because $10^{0}=1$. Lastly, the optional multiplier attribute can be used to multiply the kind unit by a real-numbered factor; this enables the definition of units that are not power-of-ten multiples of SI units. For instance, a multiplier of 0.3048 could be used to define "foot" as a measure of length in terms of a metre. The multiplier attribute has a default value of "1" (one). 
The unit system allows model quantities to be expressed in units other than the base units of Table 1. For analyses and computations, the consumer of the model (be it a software tool or a human) will want to convert all model quantities to base SI units for purposes such as verifying the consistency of units throughout the model. Suppose we begin with a quantity having numerical value $y$ when expressed in units $\{u\}$. The relationship between $y$ and a quantity $y_{b}$ expressed in base units $\left\{u_{b}\right\}$ is

$$
y_{b}\left\{u_{b}\right\}=y\{u\}\left(\frac{w\left\{u_{b}\right\}}{\{u\}}\right)
$$

The term in the parentheses on the right-hand side is a factor $w$ for converting a quantity in units $\{u\}$ to another quantity in units $\left\{u_{b}\right\}$. The ratio of units leads to canceling of $\{u\}$ in the equation above and leaves a quantity in units $\left\{u_{b}\right\}$. It remains to define this factor. In terms of the SBML unit system, it is:

$$
\{u\}=\left(\text { multiplier } \cdot 10^{\text {scale }}\left\{u_{b}\right\}\right)^{\text {exponent }}
$$

where the dot $(\cdot)$ represents simple scalar multiplication. The variables multiplier, scale, and exponent in the equation above correspond to the attributes with the same names in the Unit object defined in Figure 12. The exponent in the equation above may make it more difficult to grasp the relationship immediately; so let us suppose for the moment that exponent=" 1 ". Then, it is easy to see that

$$
\{u\}=\text { multiplier } \cdot 10^{\text {scale }}\left\{u_{b}\right\}
$$

Dividing both sides by $\{u\}$ produces the ratio in the parenthesized portion of Equation 1, which means that $w=$ multiplier $\cdot 10^{\text {scale }}$. To take a concrete example, one foot expressed in terms of the metre (a base unit) requires multiplier $=$ "0.3048", exponent $=" 1 "$, and scale $=$ " 0 ":

$$
\text { foot }=0.3048 \cdot 10^{0} \cdot \text { metre }
$$

leading to a conversion between quantities of

$$
y_{b} \text { metres }=0.3048 \cdot y \text { feet }
$$

Given a quantity of, say, $y=2$, the conversion results in $y_{b}=0.6096$. To relate this to SBML terms more concretely, the following fragment of SBML illustrates how this is represented using the Unit and UnitDefinition constructs:

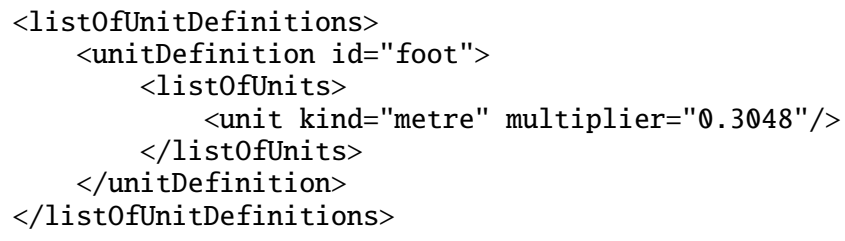

The case above is the simplest possible situation, involving the transformation of quantities from a single defined unit $\{u\}$ into a quantity expressed in a single base unit $\left\{u_{b}\right\}$. If, instead, multiple base units $\left\{u_{b_{1}}\right\},\left\{u_{b_{2}}\right\}, \ldots,\left\{u_{b_{n}}\right\}$ are involved, the following equation holds (where the $m_{i}$ terms are the multiplier values, the $s_{i}$ terms are the scale values, and the $x_{i}$ terms are the exponent values):

$$
\begin{aligned}
\{u\} & =\left(m_{1} \cdot 10^{s_{1}}\left\{u_{b_{1}}\right\}\right)^{x_{1}} \cdot\left(m_{2} \cdot 10^{s_{2}}\left\{u_{b_{2}}\right\}\right)^{x_{2}} \cdot \ldots \cdot\left(m_{n} \cdot 10^{s_{n}}\left\{u_{b_{n}}\right\}\right)^{x_{n}} \\
& =m_{1}^{x_{1}} \cdot m_{2}^{x_{2}} \cdot \ldots \cdot m_{n}^{x_{n}} \cdot 10^{\left(s_{1} x_{1}+s_{2} x_{2}+\ldots+s_{n} x_{n}\right)}\left\{u_{b_{1}}\right\}^{x_{1}}\left\{u_{b_{2}}\right\}^{x_{2}} \ldots\left\{u_{b_{n}}\right\}^{x_{n}}
\end{aligned}
$$

Software developers should take care to track the exponents carefully because they can be negative integers. The overall use of Equation 3 is analogous to that of Equation 2, and leads to the following final expression. First, to simplify, let

$$
\begin{aligned}
m & =m_{1}^{x_{1}} \cdot m_{2}^{x_{2}} \cdot \ldots \cdot m_{n}^{x_{n}} \\
p & =s_{1} x_{1}+s_{2} x_{2}+\ldots+s_{n} x_{n}
\end{aligned}
$$


then,

$$
y_{b}\left\{u_{b_{1}}\right\}\left\{u_{b_{2}}\right\} \ldots\left\{u_{b_{n}}\right\}=y\{u\}\left(\frac{m \cdot 10^{p}\left\{u_{b_{1}}\right\}^{x_{1}}\left\{u_{b_{2}}\right\}^{x_{2}} \ldots\left\{u_{b_{n}}\right\}^{x_{n}}}{\{u\}}\right)
$$

Some additional points are worth discussing about the unit scheme introduced so far. First, and most importantly, the equations above are formulated with the assumption that the base units do not require an additive offset as part of their definition. When temperature values in units other than kelvin are being considered, then a different interpretation must be made, as discussed below.

A second point is that care is needed to avoid seemingly-obvious but incorrect translations of units described in textbooks. The scheme above makes it easy to formulate statements such as " 1 foot $=0.3048$ metres" in the most natural way. However, the most common expression of the relationship between temperature in Fahrenheit and kelvin, " $T_{\text {Fahrenheit }}=1.8 \cdot\left(T_{\text {kelvin }}-273.15\right)+32$ " might lead one to believe that defining Fahrenheit degrees in terms of kelvin degrees involves using multiplier="1.8". Not so, when degree changes are being considered and not temperature values. Converting temperature values is different from expressing a relationship between degree measurements. The proper value for the multiplier in the latter case is $5 / 9$, i.e., multiplier $=0.555556$ " (where we picked an arbitrary decimal precision). If, on the other hand, the actual temperature is relevant to a quantity (e.g., if a model uses a quantity that has particular values at particular temperatures), then offsets are required in the unit calculations and a formula must be used as discussed above.

\section{Handling units requiring the use of offsets in SBML Level 2 Version 3}

Unit definitions and conversions requiring offsets cannot be done using the simple approach above. The most general case, involving offsets, multipliers and exponents, requires a completely different approach to defining units than what has been presented up to this point.

In previous versions of SBML, not only was the general case incorrectly presented (i.e., in the same terms described above, when in reality a different approach is required), but few if any developers even attempted to support offset-based units in their software. In the development of SBML Level 2 Version 2, a consensus among SBML developers emerged that a fully generalized unit scheme is so confusing and complicated that it actually impedes interoperability. Level 2 Version 2 and Version 3 acknowledge this reality by reducing and simplifying the unit system, specifically by removing the offset attribute on Unit and Celsius as a pre-defined unit, and by describing approaches for handling Celsius and other temperature units. This is a backwards-incompatible change relative to SBML Level 2 Version 1 and SBML Level 1 Version 2, but it is believed to have limited real-life impact because so few tools and models appeared to have employed this feature anyway. By simplifying the unit system to the point that it only involves multiplicative factors as described above, we expect that more software tools will be able to support the SBML unit system from this point forward, ultimately improving interoperability.

We first address the question of how to handle units that do require offsets:

- Handling Celsius. A model in which certain quantities are temperatures measured in degrees Celsius can be converted straightforwardly to a model in which those temperatures are in kelvin. A software tool could do this by performing a straightforward substitution using the following relationship:

$$
T_{\text {kelvin }}=T_{\text {Celsius }}+273.15
$$

In every mathematical formula of the model where a quantity (call it $x$ ) in degrees Celsius appears, replace $x$ with $x_{k}+273.15$ where $x_{k}$ is now in kelvin. An alternative approach would be to use a FunctionDefinition to define a function encapsulating this relationship above and then using that in the rest of the model as needed. Since Celsius is a commonly-used unit, software tools could help users by providing users with the ability to express temperatures in Celsius in the tools' interfaces, and making substitutions automatically when writing out the SBML.

- Handling other units requiring offsets. The only other units requiring offsets in SBML's domain of common applications are other temperature units such as Fahrenheit. Few modern scientists employ 
Fahrenheit degrees; therefore, this is an unusual situation. The complication inherent in converting between degrees Fahrenheit and kelvin is that both a multiplier and an offset are required:

$$
T_{\text {kelvin }}=\frac{T_{F}+459.67}{1.8}
$$

One approach to handling this is to use a FunctionDefinition to define a function encapsulating the relationship above, then to substitute a call to this function wherever the original temperature in Fahrenheit appears in the model's mathematical formulas. Here is a candidate definition as an example:

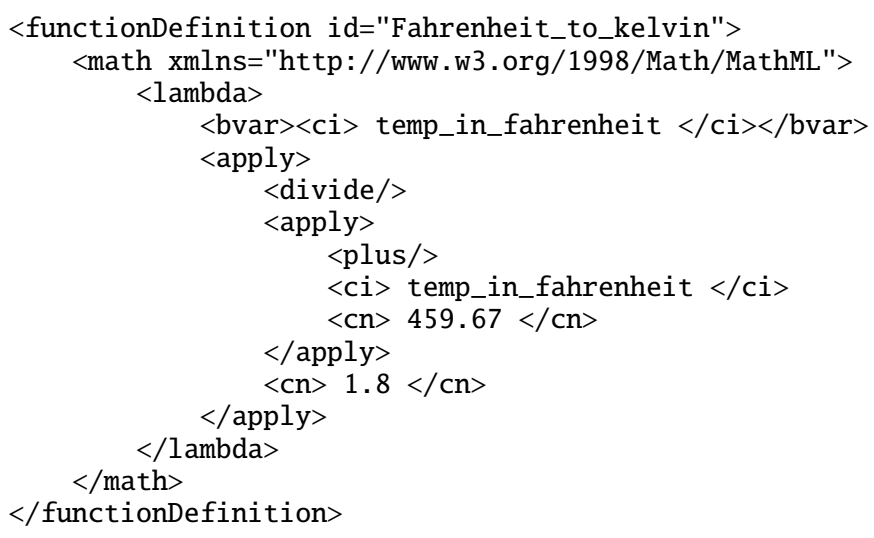

An alternative approach not requiring the use of function definitions is to use an AssignmentRule for each variable in Fahrenheit units. The AssignmentRule could compute the conversion from Fahrenheit to (say) kelvin, assign its value to a variable (in Kelvin units), and then that variable could be used elsewhere in the model. Still another approach is to rewrite the mathematical formulas of a model to directly incorporate the conversion Equation 6 wherever the quantity appears.

All of these approaches provide general solutions to the problem of supporting any units requiring offsets in the unit system of SBML Level 2 Version 2 and Version 3. It can be used for other temperature units requiring an offset (e.g., degrees Rankine, degrees Réaumur), although the likelihood of a real-life model requiring such other temperature units seems exceedingly small.

In summary, the removal of offset does not impede the creation of models using alternative units. If conversions are needed, then converting between temperature in degrees Celsius and thermodynamic temperature can be handled rather easily by the simple substitution described above. For the rarer case of Fahrenheit and other units requiring combinations of multipliers and offsets, users are encouraged to employ the power of FunctionDefinition, AssignmentRule, or other constructs in SBML.

\section{Examples}

The following example illustrates the definition of an abbreviation "mmls" for the units $m m o l l^{-1} s^{-1}$ :

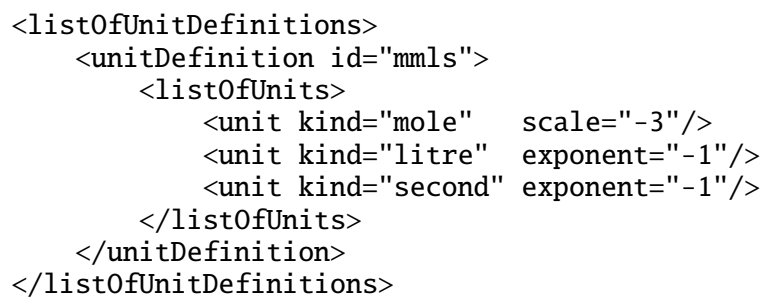

\subsubsection{Built-in units}

There are five special unit identifiers in SBML, listed in Table 2 on the next page, corresponding to the five types of quantities that can play roles in SBML reactions: substance, volume, area, length and time. All 
SBML mathematical entities apart from parameters have default units drawn from these predefined values. Table 2 lists the default values; all of the defaults have multiplier="1" and scale="0".

\begin{tabular}{lll}
\hline Identifier & Possible Scalable Units & Default Units \\
\hline substance & mole, item, gram, kilogram, dimensionless & mole \\
volume & litre, cubic metre, dimensionless & litre \\
area & square metre, dimensionless & square metre \\
length & metre, dimensionless & metre \\
time & second, dimensionless & second \\
\hline
\end{tabular}

Table 2: SBML's built-in units. The identifiers in the left-most column are values of UnitSId (Section 3.1.8).

\section{Redefinition of built-in units}

Table 2 also lists alternative base units that are allowed as the basis of redefined values. For example, a redefinition of the built-in unit of time must be based on units of seconds. Within certain limits, a model may change the built-in units by reassigning the keywords substance, length, area, time, and volume in a UnitDefinition. The limitations on redefinitions of base units are the following:

1. The UnitDefinition of a redefined built-in unit can only contain a single Unit object within it.

2. The value of the kind attribute in a Unit instance must be drawn from one of the values in the second column of the appropriate row of Table 2.

\section{Examples}

The following example illustrates how to change the built-in units of volume to be millilitres. If this definition appeared in a model, the units of volume on all components that did not explicitly specify different units would be changed to millilitres.

$<$ model $>$

...

$<$ list0fUnitDefinitions $>$

<unitDefinition id="volume">

$<$ list0fUnits $>$

<unit kind="litre" scale="-3"/>

$</$ list0fUnits $>$

$</$ unitDefinition $>$

$</$ list0fUnitDefinitions $>$

...

$</$ model $>$

\subsubsection{References to units}

An attribute that defines the units of a mathematical entity (e.g., the attribute units on Parameter) can refer to a defined unit whose identifier is chosen from among the following:

- A new unit identifier defined by a UnitDefinition as described at the start of Section 4.4;

- The base units listed in Table 1 on page 37; and

- The built-in units defined in Section 4.4.3 and listed in Table 2. (These are "substance", "volume", "area", "length", and "time".)

Software developers are asked to pay special attention to the units used in an SBML model. Different users and developers sometimes are accustomed to making different assumptions about units, and these assumptions may not correspond to what is actually defined in SBML. The numerical values in a model become meaningless if the corresponding units are not those being assumed. Sections 3.4.3, 4.8.5 and 4.13.5 have particularly important notes about the usage of units in SBML. 


\subsection{Compartment types}

A compartment type in SBML is a grouping construct used to establish a relationship between multiple compartments (Section 4.7). A compartment type is represented by the CompartmentType object class, defined in Figure 13.

In SBML Level 2 Version 3, a compartment type only has an identity, and this identity can only be used to indicate that particular compartments belong to this type. This may be useful for conveying a modeling intention, such as when a model contains many similar compartments, either by their biological function or the reactions they carry; without a compartment type construct, it would be impossible in the language of SBML to indicate that all of the compartments share an underlying conceptual relationship because each SBML compartment must be given a unique and separate identity. Compartment types have no mathematical meaning in SBML Level 2 Version 3- they have no effect on a model's mathematical interpretation. Simulators and other numerical analysis software may ignore CompartmentType objects and references to them in a model.

There is no mechanism in SBML for representing hierarchies of compartment types. One CompartmentType object instance cannot be the subtype of another CompartmentType object; SBML provides no means of defining such relationships.

\subsubsection{The id and name attributes}

As with other major class of objects in SBML, CompartmentType has a mandatory attribute, id, used to give the compartment type an identifier. The identifier must be a text string conforming to the syntax permitted by the SId data type described in Section 3.1.7. CompartmentType also has an optional name attribute, of type string. The name and id attributes must be used as described in Section 3.3.

\subsubsection{The sboTerm attribute}

CompartmentType inherits an optional sboTerm attribute of type SBOTerm from its parent class Sbase (see Sections 3.1.9 and 5). When a value is given to this attribute in a CompartmentType instance, the value must be an SBO identifier referring to a participant physical type (i.e., terms derived from SB0:0000236, "participant physical type"). The relationship is of the form "the compartment type is a X", where X is the SBO term. The term chosen should be the most precise (narrow) one that captures the role of the compartment type in the model.

\subsubsection{Examples}

The following partial SBML example illustrates a compartment type used to relate together many individual compartments in a hypothetical model.

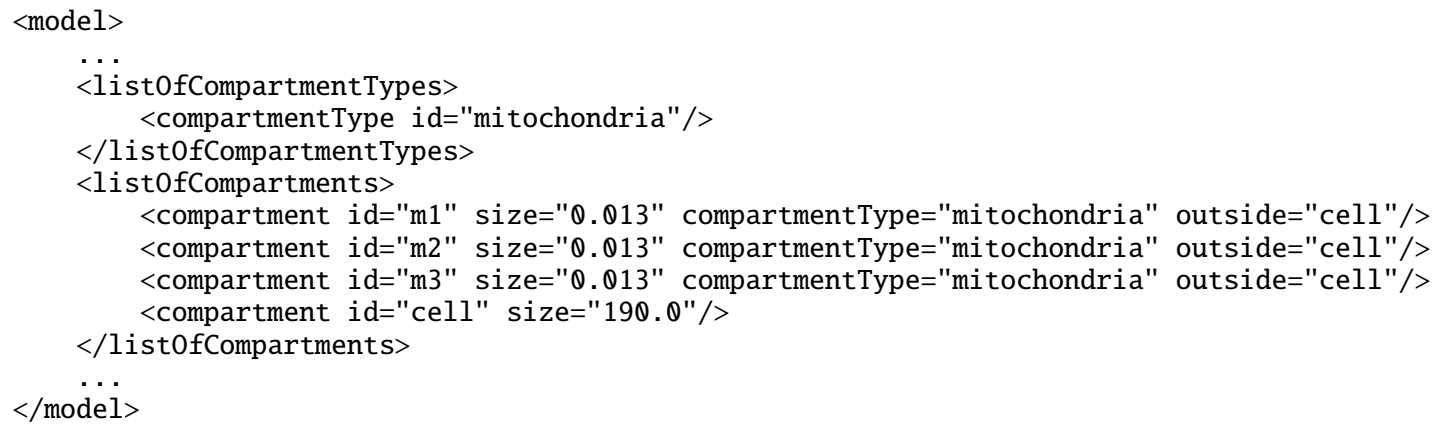




\subsection{Species types}

The term species type refers to reacting entities independent of location. These include simple ions (e.g., protons, calcium), simple molecules (e.g., glucose, ATP), large molecules (e.g., RNA, polysaccharides, and proteins), and others. The SpeciesType object class is intended to represent these entities. Its definition is shown in Figure 14.

SpeciesType objects are included in SBML to enable Species (Section 4.8) of the same type to be related together. It is a conceptual construct; the existence of SpeciesType objects in a model has no effect on the model's numerical interpretation. Except for the requirement for uniqueness of species/species type combinations located in compartments (described in Section 4.8.2), simulators and other numerical analysis software may ignore SpeciesType structures and references to them in a model.

There is no mechanism in SBML for representing hierarchies of species types. One SpeciesType object instance cannot be the subtype of another SpeciesType; SBML provides no means of defining such relationships.

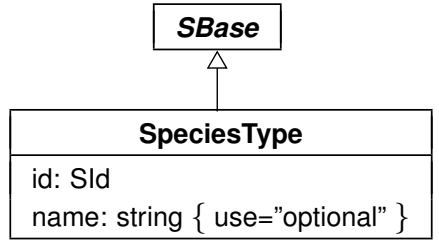

Figure 14: The definition of class Speciestype. A sequence of one or more instances of SpeciesType objects can be located in an instance of ListOfSpeciesTypes in Model, as shown in Figure 10.

\subsubsection{The id and name attributes}

As with other major object classes in SBML, SpeciesType has a mandatory attribute, id, used to give the species type an identifier. The identifier must be a text string conforming to the syntax permitted by the SId data type described in Section 3.1.7. SpeciesType also has an optional name attribute, of type string. The name and id attributes must be used as described in Section 3.3.

\subsubsection{The sboTerm attribute}

SpeciesType inherits an optional sboTerm attribute of type SBOTerm from its parent class Sbase (see Sections 3.1.9 and 5). When a value is given to this attribute in a SpeciesType instance, the value must be an SBO identifier referring to a participant physical type (i.e., terms derived from SB0:0000236, "participant physical type"). The relationship is of the form "the species type is a X", where X is the SBO term. The term chosen should be the most precise (narrow) one that captures the role of the compartment type in the model.

\subsubsection{Example}

The following XML fragment is an example of two SpeciesType objects embedded in an SBML model.

$<$ model $>$

$\ldots$

$<$ list0fSpeciesTypes $>$

$<$ speciesType id="ATP"/>

$</$ listOfSpeciesTypes $>$

$<$ list0fCompartments $>$

<compartment id="cytosol"/>

$<$ compartment id="mitochon"/>

$</$ list0fCopartments $>$

$<$ list0fSpecies $>$

<species id="ATPc" speciesType="ATP" compartment="cytosol" initialConcentration="1"/>

<species id="ATPm" speciesType="ATP" compartment="mitochon" initialConcentration="2"/> $</$ listOfSpecies $>$

$<$ model $>$ 


\subsection{Compartments}

A compartment in SBML represents a bounded space in which species are located. Compartments do not necessarily have to correspond to actual structures inside or outside of a biological cell, although models are often designed that way. The definition of Compartment is shown in Figure 15.

\begin{tabular}{|l|}
\hline \multicolumn{1}{|c|}{ SBase } \\
\hline id: SId \\
name: string $\{$ use="optional" $\}$ \\
compartmentType: SId $\{$ use="optional" $\}$ \\
spatialDimensions: int $\{$ maxlnclusive="3" minlnclusive="0" use="optional" default="3" $\}$ \\
size: double $\{$ use="optional" $\}$ \\
units: UnitSId $\{$ use="optional" $\}$ \\
outside: SId $\{$ use="optional" $\}$ \\
constant: boolean $\{$ use="optional" default="true" $\}$ \\
\hline
\end{tabular}

Figure 15: The definition of class Compartment. A sequence of one or more instances of Compartment objects can be located in an instance of ListOfCompartments in Model, as shown in Figure 10.

It is important to note that although compartments are optional in the overall definition of Model (see Section 4.2), every species in an SBML model must be located in a compartment. This in turn means that if a model defines any species, the model must also define at least one compartment. The reason is simply that species represent physical things, and therefore must exist somewhere. Compartments represent the somewhere.

\subsubsection{The id and name attributes}

Compartment has one required attribute, id, of type SId, to give the compartment a unique identifier by which other parts of an SBML model definition can refer to it. A compartment can also have an optional name attribute of type string. Identifiers and names must be used according to the guidelines described in Section 3.3.

\subsubsection{The compartmentType attribute}

Each compartment in a model may optionally be designated as belonging to a particular compartment type. The optional attribute compartmentType of type SId is used identify the compartment type represented by the Compartment object. The compartmentType attribute's value must be the identifier of a CompartmentType instance defined in the model. If the compartmentType attribute is not present on a particular compartment definition, a unique virtual compartment type is assumed for that compartment, and no other compartment can belong to that compartment type.

The values of compartmentType attributes on compartments have no effect on the numerical interpretation of a model. Simulators and other numerical analysis software may ignore compartmentType attributes.

\subsubsection{The spatialDimensions attribute}

A Compartment object has an optional attribute spatialDimensions, whose value must be a positive integer indicating the number of spatial dimensions possessed by the compartment. The maximum value is "3", meaning a three-dimensional structure (a volume). Other permissible values are "2" (for a two-dimensional area), "1" (for a one-dimensional curve), and " 0 " (for a point). The default value is " 3 ". Note that the number of spatial dimensions possessed by a compartment affects certain aspects of the compartment's size and units-of-size; see the following two subsections. 


\subsubsection{The size attribute}

Each compartment has an optional floating-point attribute named size, representing the initial total size of the compartment. The size may be a volume (if the compartment is a three-dimensional one), or it may be an area (if the compartment is two-dimensional), or a length (if the compartment is one-dimensional).

It is important to note that in SBML Level 2, a missing size value does not imply that the compartment size is 1. There is no default value of compartment size. (This is unlike the definition of compartment volume in SBML Level 1.) When the spatialDimensions attribute does not have a value of " 0 ", a missing value for size for a given compartment signifies that the value either is unknown, or to be obtained from an external source, or determined by an initial assignment (Section 4.10) or a rule (Section 4.11) elsewhere in the model. The size attribute must not be present if the spatialDimensions attribute has a value of "0"; otherwise, a logical inconsistency would exist because a zero-dimensional object cannot have a physical size.

A compartment's size is set by its size attribute exactly once. If the compartment's constant attribute value is "true" (the default), then the size is fixed and cannot be changed except by an InitialAssignment in the model (and if spatialDimensions=" 0 ", it cannot be changed by any InitialAssignment either). These methods of setting the size differ in that the size attribute can only be used to set the compartment size to a literal scalar value, whereas InitialAssignment allows the value to be set using an arbitrary mathematical expression. If the compartment's constant attribute is "false", the size value may be overridden by an InitialAssignment or changed by an AssignmentRule or AlgebraicRule, and in addition, for simulation time $t>0$, it may also be changed by a RateRule or Events. (However, some constructs are mutually exclusive; see Sections 4.11 and 4.14.) It is not an error to define size on a compartment and also redefine the value using an InitialAssignment, but the size value in that case is ignored. Section 3.4.8 provides additional information about the semantics of assignments, rules and values for simulation time $t \leq 0$.

For the reasons given above, the size attribute on a compartment must be defined as optional; however, it is extremely good practice to specify values for compartment sizes when such values are available. There are two major technical reasons for this. First, models ideally should be instantiable in a variety of simulation frameworks. A commonly-used one is the discrete stochastic framework (Gillespie, 1977; Wilkinson, 2006) in which species are represented as item counts (e.g., molecule counts). If species' initial quantities are given in terms of concentrations or densities, it is impossible to convert the values to item counts without knowing compartment sizes. Second, and more importantly, if a model contains multiple compartments whose sizes are not all identical to each other, it is impossible to quantify the reaction rate expressions without knowing the compartment volumes. The reason for the latter is that reaction rates in SBML are defined in terms of substance/time (see Section 4.13.5), and when species quantities are given in terms of concentrations or densities, the compartment sizes become factors in the reaction rate expressions.

\subsubsection{The units attribute}

The units associated with the compartment's size value may be set using the optional attribute units. The default units, and the kinds of units allowed as values of the attribute units, interact with the number of spatial dimensions of the compartment. The value of the units attribute of a Compartment object must be one of the base units from Table 1, or the built-in units "volume", "area", "length" or "dimensionless", or a new unit defined by a unit definition in the enclosing model, subject to the restrictions detailed in Table 3.

\begin{tabular}{cllll}
\hline $\begin{array}{c}\text { Value of attribute } \\
\text { spatialDimensions }\end{array}$ & $\begin{array}{l}\text { size } \\
\text { allowed? }\end{array}$ & $\begin{array}{l}\text { units } \\
\text { allowed? }\end{array}$ & Allowable kinds of units & $\begin{array}{l}\text { Default value } \\
\text { of attribute units }\end{array}$ \\
\hline "3" & yes & yes & units of volume, or dimensionless & "volume" \\
"2" & yes & yes & units of area, or dimensionless & "area" \\
"1" & yes & yes & units of length, or dimensionless & "length" \\
"0" & no & no & (no units allowed) & \\
\hline
\end{tabular}

Table 3: The units permitted for compartment sizes. If spatialDimensions=" 0 ", the compartment's units attribute must be left unset. Units of volume means litres, cubic metres, or units derived from them; units of area means square metres or units derived from square metres; and units of length means metres or units derived from metres. (See also Table 2 on page 41 and Table 1 on page 37.) 
The units of the compartment size, as defined by the units attribute or (if units is not set) the default value listed in Table 3 on the preceding page, are used in the following ways when the compartment has a spatialDimensions value greater than "O":

- The value of the units attribute is used as the units of the compartment identifier when the identifier appears as a numerical quantity in a mathematical formula expressed in MathML (discussed in Section 3.4.3).

- The math element of an AssignmentRule or InitialAssignment referring to this compartment must have identical units (see Sections 4.11.3 and 4.10).

- In RateRule objects that set the rate of change of the compartment's size (Section 4.11.4), the units of the rule's math element must be identical to the compartment's units attribute divided by the default time units. (In other words, the units for the rate of change of compartment size are compartment size/time units.)

- When a Species is to be treated in terms of concentrations or density, the units of the spatial size portion of the concentration value (i.e., the denominator in the units formula substance/size) are those indicated by the value of the units attribute on the compartment in which the species is located.

Compartments with spatialDimensions= "0" require special treatment in this framework. If a compartment has no size or dimensional units, how should such a compartment's identifier be interpreted when it appears in mathematical formulas? The answer is that such a compartment's identifier should not appear in mathematical formulas in the first place - it has no value, and its value cannot change (Section 4.7.6). Note also that a zero-dimensional compartment is a point, and species located at points can only be described in terms of amounts, not spatially-dependent measures such as concentration. Since SBML KineticLaw formulas are already in terms of substance/time and not (say) concentration/time, volume or other factors in principle are not needed for species located in zero-dimensional compartments.

\subsubsection{The constant attribute}

A Compartment also has an optional boolean attribute called constant that indicates whether the compartment's size stays constant or can vary during a simulation. A value of "false" indicates the compartment's size can be changed by other constructs in SBML. A value of "true" indicates the compartment's size cannot be changed by any other construct except InitialAssignment. In the special case of spatialDimensions=" 0 ", the value cannot be changed by InitialAssignment either. The default value for the constant attribute is "true" because in the most common modeling scenarios at the time of this writing, compartment sizes remain constant. The constant attribute must default to or be set to "true" if the value

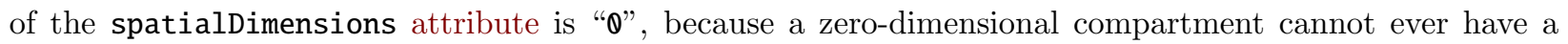
size.

\subsubsection{The outside attribute}

The optional attribute outside of type SId can be used to express containment relationships between compartments. If present, the value of outside for a given compartment must be the id attribute value of another compartment already defined in the model. Doing so means that the other compartment contains it or is "outside" of it. This enables the representation of simple topological relationships between compartments, for those simulation systems that can make use of the information (e.g., for drawing simple diagrams of compartments).

There are two restrictions on the containment relationships in SBML. First, because a compartment with spatialDimensions of " 0 " has no size, such a compartment cannot act as the container of any other compartment except compartments that also have spatialDimensions values of "0". Second, the directed graph formed by representing Compartment objects as vertexes and the outside attribute values as edges must be acyclic. The latter condition is imposed to prevent a compartment from being contained inside itself.

Although containment relationships are partly taken into account by the compartmental localization of reactants and products, it is not always possible to determine purely from the reaction equations whether 
one compartment is meant to be located within another. In the absence of a value for outside, compartment definitions in SBML Level 2 do not have any implied spatial relationships between each other. For many modeling applications, the transfer of substances described by the reactions in a model sufficiently express the relationships between the compartments. (As discussed in Section 8.1, SBML Level 3 is expected introduce the ability to define geometries and spatial qualities.)

\subsubsection{The sboTerm attribute}

Compartment inherits an optional sboTerm attribute of type SBOTerm from its parent class Sbase (see Sections 3.1.9 and 5). When a value is given to this attribute in a Compartment instance, the value must be an SBO identifier referring to a participant physical type (i.e., terms derived from SB0:0000236, "participant physical type"). The relationship is of the form "the compartment is a X", where X is the SBO term. The term chosen should be the most precise (narrow) one that captures the role of the compartment type in the model.

\subsubsection{Examples}

The following example illustrates two compartments in an abbreviated SBML example of a model definition:

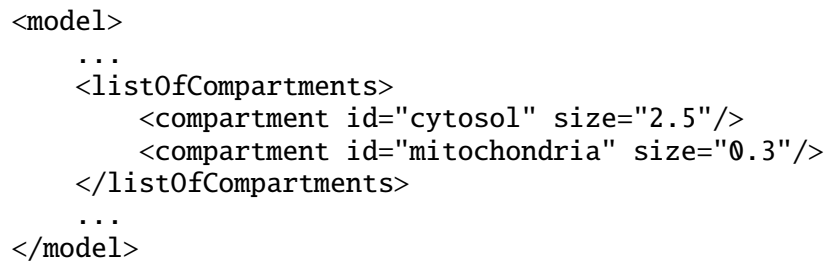

The following is an example of using outside to model a cell membrane. To express that a compartment with identifier " $\mathrm{B}$ " has a membrane that is modeled as another compartment " $\mathrm{N}$ ", which in turn is located within another compartment "A", one would write:

$<$ model $>$

$\ldots$

$<$ list0fCompartments $>$

\subsection{Species}

A species refers to a pool of reacting entities of a specific species type that take part in reactions and are located in a specific compartment. The Species object class is intended to represent these pools. Its definition is shown in Figure 16 on the next page. Although the exact definition of Species given here has changed from the definition in the specification of SBML Level 2 Version 1 (i.e., through the introduction of SpeciesType), the concept represented by Species remains the same.

In previous versions of SBML Level 2, the class Species included an attribute called spatialSizeUnits, which allowed explicitly setting the units of size for initial concentration. SBML Level 2 Version 3 removes this attribute for two reasons. First, its semantics were confusing and introduced an implicit unit conversion. Compartment has its own size-setting attribute, and a separate attribute on Species meant that a species' initial concentration could involve spatial size units that are different from the units of the compartment in which the species was located. Since (a) the spatialSizeUnits attribute determined the size units of a species' quantity when that species appeared in reaction rate formulas, and (b) the compartment may have its own different spatial units, when both the species and the compartment appeared in reaction rate formulas, one of the quantities would have had to be converted into the same spatial units as the other. In other words, modelers and software tools would have had to insert explicit conversion factors into kinetic 


\begin{tabular}{|l|}
\multicolumn{1}{c|}{ SBase } \\
\hline \multicolumn{1}{|c|}{ Species } \\
\hline id: SId \\
name: string $\{$ use="optional" $\}$ \\
speciesType: SId $\{$ use="optional" $\}$ \\
compartment: SId \\
initialAmount: double $\{$ use="optional" $\}$ \\
initialConcentration: double $\{$ use="optional" $\}$ \\
substanceUnits: UnitSId $\{$ use="optional" $\}$ \\
hasOnlySubstanceUnits: boolean $\{$ use="optional" default="false" $\}$ \\
boundaryCondition: boolean $\{$ use="optional" default="false" $\}$ \\
charge: int $\{$ use="optional" $\}$ deprecated \\
constant: boolean $\{$ use="optional" default="false" $\}$ \\
\hline
\end{tabular}

Figure 16: The definition of class Species. A sequence of one or more instances of Species objects can be located in an instance of ListOfSpecies in Model, as shown in Figure 10.

rate formulas. Such conversions can be be difficult to achieve and prone to errors. Second, although the spatialSizeUnits attribute provided some new functionality, it could also be argued to be redundant: a compartment's definition logically should be the entity controlling its own spatial size information, in the same way that a species' definition controls its quantity. For these reasons, and because few software packages seemed to take account of the implicit unit conversion, it was deemed better to remove the spatialsizeUnits attribute from Species.

\subsubsection{The id and name attributes}

As with other major objects in SBML, Species has a mandatory attribute, id, used to give the species an identifier. The identifier must be a text string conforming to the syntax permitted by the SId data type described in Section 3.1.7. Species also has an optional name attribute, of type string. The name and id attributes must be used as described in Section 3.3.

\subsubsection{The speciesType attribute}

Each species in a model may optionally be designated as belonging to a particular species type. The optional attribute speciesType of type SId is used to identify the species type of the chemical entities that make up the pool represented by the Species object. The attribute's value must be the identifier of an existing SpeciesType object. If the speciesType attribute is not present on a particular species definition, it means the pool contains chemical entities of a type unique to that pool; in effect, a virtual species type is assumed for that species, and no other species can belong to that species type.

There can be only one species of a given species type in any given compartment of a model. More specifically, for all Species objects having a value for the speciesType attribute, the pair

(speciesType attribute value, compartment attribute value)

must be unique across the set of all Species objects in a model.

The value of speciesType attributes on species have no effect on the numerical interpretation of a model. Simulators and other numerical analysis software may ignore speciesType attributes.

\subsubsection{The compartment attribute}

The required attribute compartment, also of type SId, is used to identify the compartment in which the species is located. The attribute's value must be the identifier of an existing Compartment object. It is important to note that there is no default value for the compartment attribute on Species; every species in an SBML model must be assigned a compartment, and consequently, a model must define at least one compartment if that model contains any species. 


\subsubsection{The initialAmount and initialConcentration attributes}

The optional attributes initialAmount and initialConcentration, both having a data type of double, are used to set the initial quantity of the species in the compartment where the species is located. These attributes are mutually exclusive; i.e., only one can have a value on any given instance of a Species object.

Missing initialAmount and initialConcentration values implies that their values either are unknown, or to be obtained from an external source, or determined by an initial assignment (Section 4.10) or rule (Section 4.11) elsewhere in the model. In the case where a species' compartment has a spatialDimensions value of " $\nabla$ ", the species cannot have a value for initialConcentration because the concepts of "concentration" and "density" break down when a container has zero dimensions.

A species' initial quantity is set by the initialAmount or initialConcentration attributes exactly once. If the species' constant attribute is "true" (the default), then the size is fixed and cannot be changed except by an InitialAssignment. These methods differ in that the initialAmount and initialConcentration attributes can only be used to set the species quantity to a literal scalar value, whereas InitialAssignment allows the value to be set using an arbitrary mathematical expression. If the species' constant attribute is "false", the species' quantity value may be overridden by an InitialAssignment or changed by AssignmentRule or AlgebraicRule, and in addition, for $t>0$, it may also be changed by a RateRule or Events. (However, some constructs are mutually exclusive; see Sections 4.11 and 4.14.) It is not an error to define initialAmount or initialConcentration on a species and also redefine the value using an InitialAssignment, but the initialAmount or initialConcentration setting in that case is ignored. Section 3.4.8 provides additional information about the semantics of assignments, rules and values for simulation time $t \leq 0$.

The units of the value in the initialAmount attribute are set by the substanceUnits attribute on Species. The units of the value in the initialConcentration attribute are substance/size units. The units of substance are those defined in the substanceUnits, and the size units are those given in the definition of the size of the Compartment in which the species is located.

\subsubsection{The substanceUnits and hasOnlySubstanceUnits attributes}

The units associated with a species' quantity, referred to as the units of the species, are determined via the optional attributes substanceUnits and hasOnlySubstanceUnits, in combination with the units of the size defined for the compartment object in which the species are located.

The attribute hasOnlySubstanceUnits takes on boolean values and defaults to "false". This attribute's role is to indicate whether the units of the species, when the species identifier appears in mathematical formulas, are intended to be concentration or amount. Although it may seem as though this intention could be determined based on whether initialConcentration or initialAmount is set, the fact that these two attributes are optional means that a separate flag is needed. (Consider the situation where neither is set, and instead the species' quantity is established by an InitialAssignment or AssignmentRule.)

The possible values of units of the species are summarized in Table 4. The units of the species are of the form substance/size units (i.e., concentration units, using a broad definition of concentration) if the compartment's spatialDimensions is non-zero and hasOnlySubstanceUnits has the value "false". The units of the species are of the form substance if hasOnlySubstanceUnits has the value "true" or spatialDimensions is zero. (This dependence is due to the fact that a zero-dimensional compartment cannot support concentrations or densities.) The units of substance are those defined by the substanceUnits attribute, and the size units are those of the size of the compartment in which the species is located. This compartment is the one identified by the species' compartment attribute.

\begin{tabular}{lll}
\hline $\begin{array}{l}\text { value of } \\
\text { hasOnlySubstanceUnits }\end{array}$ & $\begin{array}{l}\text { units of the species } \text { when } \\
\text { spatialDimensions is greater than } \mathbf{0}\end{array}$ & $\begin{array}{l}\text { units of the species } \text { when } \\
\text { spatialDimensions is } \mathbf{0}\end{array}$ \\
\hline false (default) & $\begin{array}{l}\text { substance/size } \\
\text { substance }\end{array}$ & $\begin{array}{l}\text { substance } \\
\text { true }\end{array}$ \\
\hline
\end{tabular}

Table 4: How to interpret the value the Species hasOnlySubstanceUnits attribute. 
As an aside, we note that treating species in terms of substance units (i.e., discrete quantities such as molecule counts) rather than concentrations is common when using discrete stochastic simulation frameworks (Gillespie, 1977; Wilkinson, 2006). The appropriate way of accomplishing this in SBML is to set has0nlySubstanceUnits= "true" in the species' definitions.

The value chosen for substanceUnits must be either a base unit from Table 1 on page 37, a built-in unit from Table 2 on page 41, or a new unit defined by a unit definition in the enclosing model. The chosen units for substanceUnits must be be dimensionless, mole, item, kilogram, gram, or units derived from these. The substanceUnits attribute defaults to the the built-in unit "substance" shown in Table 2 on page 41.

The units of the species are used in the following ways:

- The species identifier has these units when the identifier appears as a numerical quantity in a mathematical formula expressed in MathML (discussed in Section 3.4.3).

- The math element of an AssignmentRule or InitialAssignment referring to this species must have identical units (see Sections 4.11.3 and 4.10).

- In RateRule objects that set the rate of change of the species' quantity (Section 4.11.4), the units of the rule's math element must be identical to the units of the species divided by the model's time units.

\subsubsection{The constant and boundaryCondition attributes}

The Species object has two optional boolean attributes named constant and boundaryCondition, used to indicate whether and how the quantity of that species can vary during a simulation. Table 5 shows how to interpret the combined values of the boundaryCondition and constant attributes.

\begin{tabular}{lllll}
\hline $\begin{array}{l}\text { constant } \\
\text { value }\end{array}$ & $\begin{array}{l}\text { boundaryCondition } \\
\text { value }\end{array}$ & $\begin{array}{l}\text { can have } \\
\text { assignment } \\
\text { or rate rule }\end{array}$ & $\begin{array}{l}\text { can be } \\
\text { reactant } \text { or } \\
\text { product }\end{array}$ & $\begin{array}{l}\text { species' quantity } \\
\text { can be changed by }\end{array}$ \\
\hline true & true & no & yes & (never changes) \\
false & true & yes & yes & rules and events \\
true & false & no & no & (never changes) \\
false & false & yes & yes & reactions or rules (but not both), and events \\
\hline
\end{tabular}

Table 5: How to interpret the values of the constant and boundaryCondition attributes on Species. Note that column four is specifically about reactants and products and not also about species acting as modifiers; the latter are by definition unchanged by reactions.

By default, when a species is a product or reactant of one or more reactions, its quantity is determined by those reactions. In SBML, it is possible to indicate that a given species' quantity is not determined by the set of reactions even when that species occurs as a product or reactant; i.e., the species is on the boundary of the reaction system, and its quantity is not determined by the reactions. The boolean attribute boundaryCondition can be used to indicate this. The value of the attribute defaults to "false", indicating the species is part of the reaction system.

The constant attribute indicates whether the species' quantity can be changed at all, regardless of whether by reactions, rules, or constructs other than InitialAssignment. The default value is "false", indicating that the species' quantity can be changed, since the purpose of most simulations is precisely to calculate changes in species quantities. Note that the initial quantity of a species can be set by an InitialAssignment irrespective of the value of the constant attribute.

In practice, a boundaryCondition value of "true" means a differential equation derived from the reaction definitions should not be generated for the species. However, the species' quantity may still be changed by AssignmentRule, RateRule, AlgebraicRule, Event, and InitialAssignment constructs if its constant attribute is "false". Conversely, if the species' constant attribute is "true", then its value cannot be changed by anything except InitialAssignment. 
A species having boundaryCondition="false" and constant="false" can appear as a product and/or reactant of one or more reactions in the model. If the species is a reactant or product of a reaction, it must not also appear as the target of any AssignmentRule or RateRule object in the model. If instead the species has boundaryCondition="false" and constant="true", then it cannot appear as a reactant or product, or as the target of any AssignmentRule, RateRule or EventAssignment object in the model.

The example model in section 7.6 contains all four possible combinations of the boundaryCondition and constant attributes on species elements. Section 7.7 gives an example of how one can translate into ODEs a model that uses boundaryCondition and constant attributes.

\subsubsection{The charge attribute}

The optional attribute charge takes an integer indicating the charge on the species (in terms of electrons, not the SI unit coulombs). This may be useful when the species is a charged ion such as calcium $\left(\mathrm{Ca}^{2+}\right)$. The charge attribute in SBML has been deprecated since Level 2 Version 2.

\subsubsection{The sboTerm attribute}

Species inherits an optional sboTerm attribute of type SBOTerm from its parent class Sbase (see Sections 3.1.9 and 5). When a value is given to this attribute in a Species instance, the value must be an SBO identifier referring to a participant physical type (i.e., terms derived from SB0:0000236, "participant physical type"). The relationship is of the form "the species is a X", where X is the SBO term. The term chosen should be the most precise (narrow) one that captures the role of the compartment type in the model.

\subsubsection{Example}

The following example shows two species definitions within an abbreviated SBML model definition. The example shows that species are listed under the heading list0fSpecies in the model:

$<$ model $>$

$<$ list0fSpecies $>$

<species id="Glucose" compartment="cell" initialConcentration="4"/>

<species id="Glucose_6_P" compartment="cell" initialConcentration="0.75"/> $</$ listOfSpecies $>$

$\ldots$

$<$ model $>$

\subsection{Parameters}

A Parameter is used in SBML to define a symbol associated with a value; this symbol can then be used in mathematical formulas in a model. By default, parameters have constant value for the duration of a simulation, and for this reason are called "parameters" instead of "variables" in SBML, although in truth, SBML parameters can be either. The definition of Parameter is shown in Figure 17.

\begin{tabular}{|l|}
\multicolumn{1}{c|}{ SBase } \\
\hline \multicolumn{1}{|c|}{ Parameter } \\
\hline id: SId \\
name: string $\{$ use="optional" $\}$ \\
value: double $\{$ use="optional" $\}$ \\
units: UnitSId \{ use="optional" $\}$ \\
constant: boolean $\{$ use="optional" default="true" $\}$ \\
\hline
\end{tabular}

Figure 17: The definition of class Parameter. A sequence of one or more instances of Parameter objects can be located in an instance of ListOfParameters in Model, as shown in Figure 10. 
Parameters can be defined in two places in SBML: in lists of parameters defined at the top level in a Model instance, and within individual reaction definitions (as described in Section 4.13). Parameters defined at the top level are global to the whole model; parameters that are defined within a reaction are local to the particular reaction and (within that reaction) override any global parameters having the same identifiers (See Section 3.3.1 for further details).

The use of the term parameter in SBML sometimes leads to confusion among readers who have a particular notion of what something called "parameter" should be. It has been the source of heated debate, but despite this, no one has yet found an adequate replacement term that does not have different connotations to different people and hence leads to confusion among some subset of users. Perhaps it would have been better to have two constructs, one called "constants" and the other called "variables". The current approach in SBML is simply more parsimonious, using a single Parameter construct with the boolean flag constant indicating which flavor it is. In any case, readers are implored to look past their particular definition of a "parameter" and simply view SBML's Parameter as a single mechanism for defining both constants and (additional) variables in a model. (We write additional because the species in a model are usually considered to be the central variables.) After all, software tools are not required to expose to users the actual names of particular SBML constructs, and thus tools can present to their users whatever terms their designers feel best matches their target audience.

\subsubsection{The id and name attributes}

Parameter has one required attribute, id, of type SId, to give the parameter a unique identifier by which other parts of an SBML model definition can refer to it. A parameter can also have an optional name attribute of type string. Identifiers and names must be used according to the guidelines described in Section 3.3.

\subsubsection{The value attribute}

The optional attribute value determines the value (of type double) assigned to the identifier. A missing value implies that the value either is unknown, or to be obtained from an external source, or determined by an initial assignment (Section 4.10) or a rule (Section 4.11) elsewhere in the model.

A parameter's value is set by its value attribute exactly once. If the parameter's constant attribute has the value "true" (the default), then the value is fixed and cannot be changed except by an InitialAssignment. These two methods of setting the parameter's value differ in that the value attribute can only be used to set it to a literal scalar value, whereas InitialAssignment allows the value to be set using an arbitrary mathematical expression. If the parameter's constant attribute has the value "false", the parameter's value may be overridden by an InitialAssignment or changed by AssignmentRule or AlgebraicRule, and in addition, for simulation time $t>0$, it may also be changed by a RateRule or Events. (However, some of these constructs are mutually exclusive; see Sections 4.11 and 4.14.) It is not an error to define value on a parameter and also redefine the value using an InitialAssignment, but the value in that case is ignored. Section 3.4.8 provides additional information about the semantics of assignments, rules and values for simulation time $t \leq 0$.

\subsubsection{The units attribute}

The units associated with the value of the parameter are specified by the attribute units. The value assigned to the parameter's units attribute must be chosen from one of the following possibilities: one of the base unit identifiers from Table 1 on page 37; one of the built-in unit identifiers appearing in first column of Table 2 on page 41; or the identifier of a new unit defined in the list of unit definitions in the enclosing Model definition. There are no constraints on the units that can be chosen from these sets. There are no default units for parameters.

The units of the parameter are used in the following ways:

- When the parameter identifier appears in mathematical formulas expressed in MathML in a model, the units associated with the value are those declared by the parameter's units attribute.

- The units of the math element of an AssignmentRule, InitialAssignment or EventAssignment setting the value of the parameter must have units identical to those declared by the parameter's units attribute. 
- The units of the math element of a RateRule that references the parameter be identical to parameter units/time, where parameter units are the units declared for the parameter using the units attribute and time is the model-wide time units.

The fact that parameter units are optional, and that no defaults are defined, means that models can define parameters with undeclared units. If such parameters appear in mathematical expressions elsewhere in a model, it may not be possible for a software tool to verify the consistency of units used in the expressions. Modelers and software tools should therefore assign units to parameters whenever possible.

\subsubsection{The constant attribute}

The Parameter object has an optional boolean attribute named constant which indicates whether the parameter's value can vary during a simulation. The attribute's default value is "true". A value of "false" indicates the parameter's value can be changed by rules (see Section 4.11) and that the value is actually intended to be the initial value of the parameter.

Parameters local to a reaction (i.e., those defined within a Reaction's KineticLaw object, as described in Section 4.13.5) cannot be changed by rules and therefore are implicitly always constant; thus, parameter definitions within Reaction objects should not have their constant attribute set to "false".

What if a global parameter has its constant attribute set to "false", but the model does not contain any rules, events or other constructs that ever change its value over time? Although the model may be suspect, this situation is not strictly an error. A value of "false" for constant only indicates that a parameter can change value, not that it must.

\subsubsection{The sboTerm attribute}

The Parameter object inherits from Sbase the optional sboTerm attribute of type SBOTerm (see Sections 3.1.9 and 5). When a value is given to this attribute in a parameter definition, the value must be an SBO identifier referring to a quantitative parameter defined in SBO (i.e., terms derived from SB0:0000002, "quantitative parameter"). The relationship is of the form "the SBML parameter is a X", where X is the SBO term. The term chosen should be the most precise (narrow) one that captures the role of the parameter in the model.

As discussed in Section 5, SBO labels are optional information on a model. Applications are free to ignore sboTerm values. A model must be interpretable without the benefit of SBO labels.

\subsubsection{Example}

The following is an example of parameters defined at the Model level:

$<$ model $>$

...

$<$ list0fParameters $>$

$<$ parameter id="tau2" value="3e-2" units="second"/>

$<$ parameter id="Km1" value="10.7" units="moleperlitre"/>

$</$ list0fParameters $>$

...

$</$ model $>$

\subsection{Initial assignments}

SBML Level 2 Version 3 provides two ways of assigning initial values to entities in a model. The simplest and most basic is to set the values of the appropriate attributes in the relevant components; for example, the initial value of a model parameter (whether it is a constant or a variable) can be assigned by setting its value attribute directly in the model definition (Section 4.9). However, this approach is not suitable when the value must be calculated, because the initial value attributes on different components such as species, compartments, and parameters are single values and not mathematical expressions. This is the reason for the introduction of InitialAssignment: to permit the calculation of the value of a constant or the initial value of a variable from the values of other quantities in a model. The definition of InitialAssignment is shown in Figure 18 on the following page. 


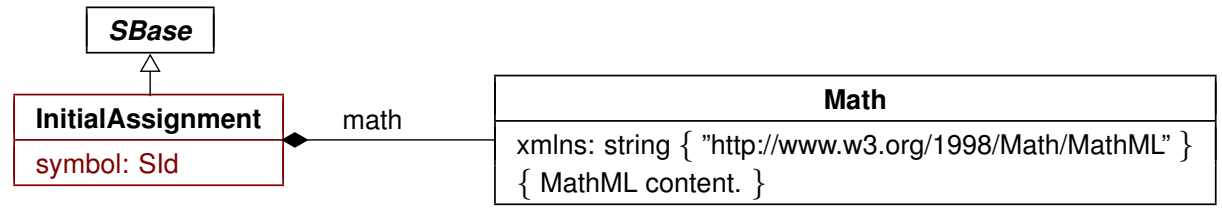

Figure 18: The definition of class InitialAssignment. The contents of the Math class can be any MathML permitted in SBML; see Section 3.4.1. A sequence of one or more instances of InitialAssignment objects can be located in an instance of ListOfInitialAssignments in Model, as shown in Figure 10.

As explained below, the provision of InitialAssignment does not mean that models necessarily must use this construct when defining initial values of quantities. If a value can be set using the relevant attribute of a component in a model, then that approach may be more efficient and more portable to other software tools. InitialAssignment should be used when the other mechanism is insufficient for the needs of a particular model.

Initial assignments have some similarities to assignment rules (Section 4.11.3). The main differences are (a) an InitialAssignment can set the value of a constant whereas an AssignmentRule cannot, and (b) unlike AssignmentRule, an InitialAssignment definition only applies up to and including the beginning of simulation time, i.e., $t \leq 0$, while an AssignmentRule applies at all times.

\subsubsection{The symbol attribute}

InitialAssignment contains the attribute symbol, of type SId. The value of this attribute in an InitialAssignment object can be the identifier (i.e., the value of the id attribute) of a Compartment, Species or global Parameter elsewhere in the model. The purpose of the InitialAssignment is to define the initial value of the constant or variable referred to by the symbol attribute. (The attribute's name is symbol rather than variable because it may assign values to constants as well as variables in a model; see Section 4.10 .4 below.)

An initial assignment cannot be made to reaction identifiers, that is, the symbol attribute value of an InitialAssignment cannot be an identifier that is the id attribute value of a Reaction object in the model. This is identical to a restriction placed on rules (see Section 4.11.5).

\subsubsection{The math element}

The math element contains a MathML expression that is used to calculate the value of the constant or the initial value of the variable. The units of the value computed by the formula in the math element must be identical to be the units associated with the identifier given in the symbol attribute. (That is, the units are the units of the species, compartment, or parameter, as appropriate for the kind of object identified by the value of symbol.)

\subsubsection{The sboTerm attribute}

InitialAssignment inherits from Sbase an optional sboTerm attribute of type SBOTerm (see Sections 3.1.9 and 5). When a value is given to this attribute in an initial assignment definition, the value must be a valid SBO identifier referring to a mathematical expression (i.e., terms derived from SB0:0000064, "mathematical expression"). The InitialAssignment object should have a "is a" relationship with the SBO term, and the term should be the most precise (narrow) term that captures the role of the InitialAssignment in the model.

As discussed in Section 5, SBO labels are optional information on a model. Applications are free to ignore sboTerm values. A model must be interpretable without the benefit of SBO labels.

\subsubsection{Semantics of initial assignments}

The value calculated by an InitialAssignment object overrides the value assigned to the given symbol by the object defining that symbol. For example, if a Compartment's size is set in its definition, and the model also contains an InitialAssignment having that compartment's id as its symbol value, then the interpretation is that the size assigned in the Compartment definition should be ignored and the value assigned based on the 
computation defined in the InitialAssignment. Initial assignments can take place for Compartment, Species and global Parameter objects regardless of the value of their constant attribute.

This does not mean that a definition of a symbol can be omitted if there is an InitialAssignment object for that symbol; the symbols must always be defined even if they are assigned a value separately. For example, there must be a Parameter definition for a given parameter if there is an InitialAssignment for that parameter.

The actions of all InitialAssignment objects are in general terms the same, but differ in the precise details depending on the type of variable being set:

- In the case of a species, an InitialAssignment sets the referenced species' initial quantity (concentration or amount of substance) to the value determined by the formula in math. (See Section 4.8.5 for an explanation of how the units of the species' quantity are determined.)

- In the case of a compartment, an InitialAssignment sets the referenced compartment's initial size to the size determined by the formula in math. The overall units of the formula must be the same as the units specified for the size of the compartment. (See Section 4.7.5 for an explanation of how the units of the compartment's size are determined.)

- In the case of a parameter, an InitialAssignment sets the referenced parameter's initial value to that determined by the formula in math. The overall units of the formula must be the same as the units defined for the parameter. (See Section 4.9.3 for an explanation of how the units of the parameter are determined.)

In the context of a simulation, initial assignments establish values that are in effect prior to and including the start of simulation time, i.e., $t \leq 0$. Section 3.4.8 provides information about the interpretation of assignments, rules, and entity values for simulation time up to and including the start time $t=0$; this is important for establishing the initial conditions of a simulation if the model involves expressions containing the delay csymbol (Section 3.4.6).

There cannot be two initial assignments for the same symbol in a model; that is, a model must not contain two or more InitialAssignment objects that both have the same identifier as their symbol attribute value. A model must also not define initial assignments and assignment rules for the same entity. That is, there cannot be both an InitialAssignment and an AssignmentRule for the same symbol in a model, because both kinds of constructs apply prior to and at the start of simulated time - allowing both to exist for a given symbol would result in indeterminism). (See also Section 4.11.5.)

The ordering of InitialAssignment objects is not significant. The combined set of InitialAssignment, AssignmentRule and KineticLaw objects form a set of assignment statements that must be considered as a whole. The combined set of assignment statements should not contain algebraic loops: a chain of dependency between these statements should terminate. (More formally, consider the directed graph of assignment statements where nodes are a model's assignment statements and directed arcs exist for each occurrence of a symbol in an assignment statement math attribute. The directed arcs in this graph start from the statement assigning the symbol and end at the statement that contains the symbol in their math elements. Such a graph must be acyclic.) Examples of valid and invalid set of assignment statements are given in Section 4.11.5.

Finally, it is worth being explicit about the expected behavior in the following situation. Suppose (1) a given symbol has a value $x$ assigned to it in its definition, and (2) there is an initial assignment having the identifier as its symbol value and reassigning the value to $y$, and (3) the identifier is also used in the mathematical formula of a second initial assignment. What value should the second initial assignment use? It is $y$, the value assigned to the symbol by the first initial assignment, not whatever value was given in the symbol's definition. This follows directly from the behavior at the defined at the beginning of this section and in Section 3.4.8: if an InitialAssignment object exists for a given symbol, then the symbol's value is overridden by that initial assignment.

\subsubsection{Example}

The following example shows how the species " $\mathrm{x}$ " can assigned the initial value $2 \times y$, where " $\mathrm{y}$ " is an identifier defined elsewhere in the model: 


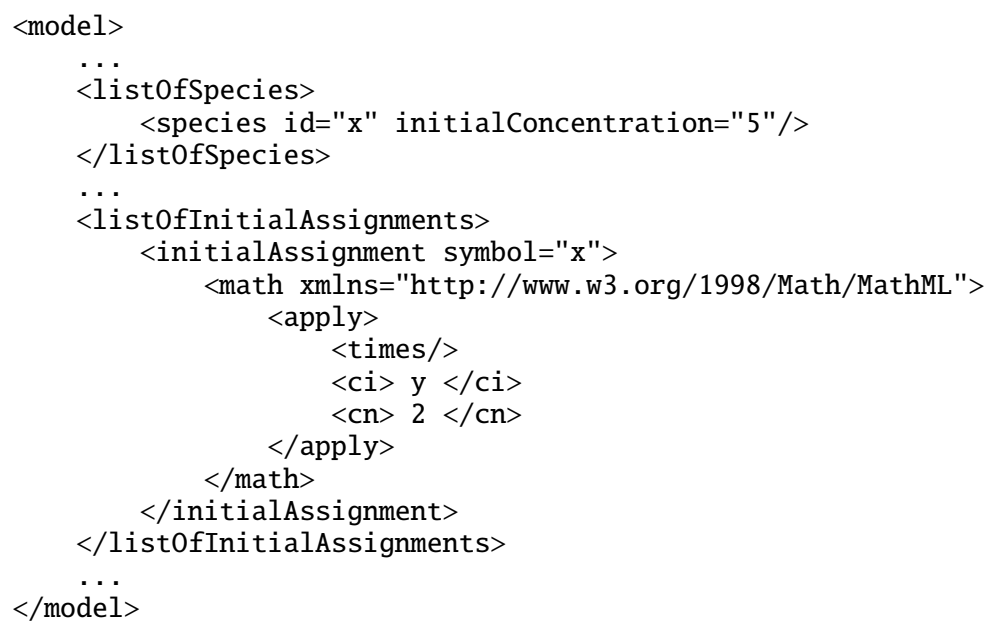

The next example illustrates the more complex behavior discussed above, when a symbol has a value assigned in its definition but there also exists an InitialAssignment for it and another InitialAssignment uses its value in its mathematical formula.

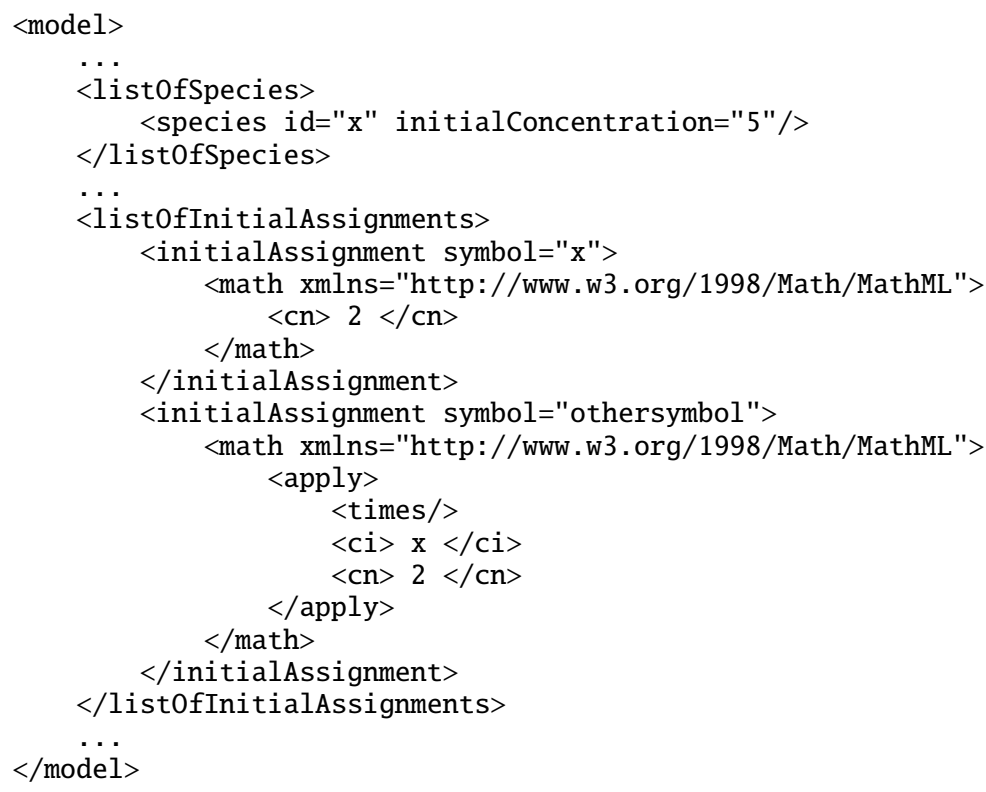

The value of "othersymbol" in the SBML excerpt above will be "4". The case illustrates the rule of thumb that if there is an initial assignment for a symbol, the value assigned to the symbol in its definition must be ignored and the value created by the initial assignment used instead.

\subsection{Rules}

In SBML, Rules provide additional ways to define the values of variables in a model, their relationships, and the dynamical behaviors of those variables. Rules enable the encoding of relationships that cannot be expressed using reactions alone (Section 4.13) nor by the assignment of an initial value to a variable in a model (Section 4.10).

SBML separates rules into three subclasses for the benefit of model analysis software. The three subclasses are based on the following three different possible functional forms (where $x$ is a variable, $f$ is some arbitrary function returning a numerical result, $\mathbf{V}$ is a vector of variables that does not include $x$, and $\mathbf{W}$ is a vector of variables that may include $x$ ): 


$\begin{array}{rll}\text { Algebraic } & \text { left-hand side is zero: } & 0=f(\mathbf{W}) \\ \text { Assignment } & \text { left-hand side is a scalar: } & x=f(\mathbf{V}) \\ \text { Rate } & \text { left-hand side is a rate-of-change: } & d x / d t=f(\mathbf{W})\end{array}$

In their general form given above, there is little to distinguish between assignment and algebraic rules. They are treated as separate cases for the following reasons:

- Assignment rules can simply be evaluated to calculate intermediate values for use in numerical methods;

- SBML needs to place restrictions on assignment rules, for example the restriction that assignment rules cannot contain algebraic loops (discussed further in Section 4.11.5);

- Some simulators do not contain numerical solvers capable of solving unconstrained algebraic equations, and providing more direct forms such as assignment rules may enable those simulators to process models they could not process if the same assignments were put in the form of general algebraic equations;

- Those simulators that can solve these algebraic equations make a distinction between the different categories listed above; and

- Some specialized numerical analyses of models may only be applicable to models that do not contain algebraic rules.

The approach taken to covering these cases in SBML is to define an abstract Rule object class containing an element, math, to hold the right-hand side expression, then to derive subtypes of Rule that add attributes to distinguish the cases of algebraic, assignment and rate rules. Figure 19 gives the definitions of Rule and the subtypes derived from it. The figure shows there are three subtypes, AlgebraicRule, AssignmentRule and RateRule derived directly from Rule. These correspond to the cases Algebraic, Assignment, and Rate described above respectively.

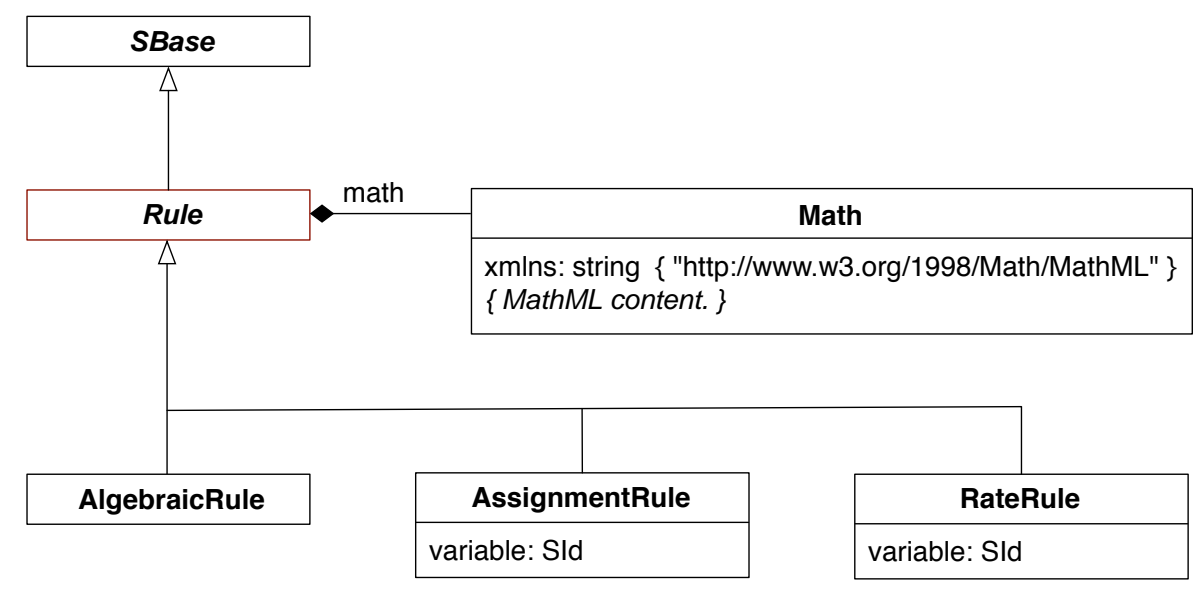

Figure 19: The definition of Rule and derived types AlgebraicRule, AssignmentRule and RateRule.

\subsubsection{Common attributes in Rule}

The classes derived from Rule inherit math and the attributes and elements from Sbase, including sboTerm.

\section{The math element}

A Rule object has a required element called math, containing a MathML expression defining the mathematical formula of the rule. This MathML formula must return a numerical value. The formula can be an arbitrary expression referencing the variables and other entities in an SBML model. The interpretation of math and the units of the formula are described in more detail in Sections 4.11.2, 4.11.3 and 4.11.4 below. 


\section{The sboTerm attribute}

The Rule object class inherits from Sbase the optional sboTerm attribute of type SBOTerm (see Sections 3.1.9 and 5). When a value is given to this attribute, it must be a valid SBO identifier referring to a mathematical expression defined in SBO (i.e., terms derived from SB0:0000064, "mathematical expression"). The AlgebraicRule, AssignmentRule, or RateRule object should have a "is a" relationship with the SBO term, and the term should be the most precise (narrow) term that captures the role of that rule in the model.

As discussed in Section 5, SBO labels are optional information on a model. Applications are free to ignore sboTerm values. A model must be interpretable without the benefit of SBO labels.

\subsubsection{AlgebraicRule}

The rule type AlgebraicRule is used to express equations that are neither assignments of model variables nor rates of change. AlgebraicRule does not add any attributes to the basic Rule; its role is simply to distinguish this case from the other cases. An example of the use of AlgebraicRule is given in Section 7.5.

In the context of a simulation, algebraic rules are in effect at all times, $t \geq 0$. For purposes of evaluating expressions that involve the delay csymbol (Section 3.4.6), algebraic rules are considered to apply also at $t \leq 0$. Section 3.4 .8 provides additional information about the semantics of assignments, rules, and entity values for simulation time $t \leq 0$.

AlgebraicRule is unlike AssignmentRule and RateRule in that there are no specific definitions of the meaning of the mathematical expression in the AlgebraicRule. There is only one requirement on the units, which is that the units of the overall expression must be consistent under dimensional analysis.

The ability to define arbitrary algebraic expressions in an SBML model introduces the possibility that a model is mathematically overdetermined by the overall system of equations constructed from its rules and reactions. An SBML model must not be overdetermined; this is discussed in Section 4.11.5 below.

\subsubsection{AssignmentRule}

The rule type AssignmentRule is used to express equations that set the values of variables. The left-hand side (the variable attribute) of an assignment rule can refer to the identifier of a Species, Compartment, or Parameter object in the model (but not a reaction). The entity identified must not have its constant attribute set to "true". The effects of an AssignmentRule are in general terms the same, but differ in the precise details depending on the type of variable being set:

- In the case of a species, an AssignmentRule sets the referenced species' quantity (concentration or amount of substance) to the value determined by the formula in math. The units of the formula in math must be the same as the units of the species (Section 4.8.5) for the species identified by the variable attribute of the AssignmentRule.

Restrictions: There must not be both an AssignmentRule variable attribute and a SpeciesReference species attribute having the same value, unless that species has its boundaryCondition attribute set to "true". In other words, an assignment rule cannot be defined for a species that is created or destroyed in a reaction unless that species is defined as a boundary condition in the model.

- In the case of a compartment, an AssignmentRule sets the referenced compartment's size to the value determined by the formula in math. The overall units of the formula in math must be the same as the units of the size of the compartment (Section 4.7.5).

- In the case of a parameter, an AssignmentRule sets the referenced parameter's value to that determined by the formula in math. The overall units of the formula in math must be the same as the units defined for the parameter (Section 4.9.3).

In the context of a simulation, assignment rules are in effect at all times, $t \geq 0$. For purposes of evaluating expressions that involve the delay csymbol (Section 3.4.6), assignment rules are considered to apply also at $t \leq 0$. Section 3.4 .8 provides additional information about the semantics of assignments, rules, and entity values for simulation time $t \leq 0$. 
A model must not contain more than one AssignmentRule or RateRule object having the same value of variable; in other words, in the set of all assignment rules and rate rules in an SBML model, each variable appearing in the left-hand sides can only appear once. This simply follows from the fact that an indeterminate system would result if a model contained more than one assignment rule for the same variable or both an assignment rule and a rate rule for the same variable.

Similarly, a model must also not contain both an AssignmentRule and an InitialAssignment for the same variable, because both kinds of constructs apply prior to and at the start of simulation time, i.e., $t \leq 0$. If a model contained both an initial assignment and an assignment rule for the same variable, an indeterminate system would result. (See also Section 4.10.4.)

The value calculated by an AssignmentRule object overrides the value assigned to the given symbol by the object defining that symbol. For example, if a Compartment's size is set in its definition, and the model also contains an AssignmentRule having that compartment's id as its variable value, then the size assigned in the Compartment definition is ignored and the value assigned based on the computation defined in the AssignmentRule. This does not mean that a definition for a given symbol can be omitted if there is an AssignmentRule object for it. For example, there must be a Parameter definition for a given parameter if there is an AssignmentRule for that parameter.

\subsubsection{RateRule}

The rule type RateRule is used to express equations that determine the rates of change of variables. The left-hand side (the variable attribute) can refer to the identifier of a species, compartment, or parameter (but not a reaction). The entity identified must have its constant attribute set to "false". The effects of a RateRule are in general terms the same, but differ in the precise details depending on which variable is being set:

- In the case of a species, a RateRule sets the rate of change of the species' quantity (concentration or amount of substance) to the value determined by the formula in math. The overall units of the formula in math must be species quantity/time, where the time units are the built-in units of time described in Section 4.4 and the species quantity units are the units of the species as defined in Section 4.8.5.

Restrictions: There must not be both a RateRule variable attribute and a SpeciesReference species attribute having the same value, unless that species has its boundaryCondition attribute is set to "true". This means a rate rule cannot be defined for a species that is created or destroyed in a reaction, unless that species is defined as a boundary condition in the model.

- In the case of a compartment, a RateRule sets the rate of change of the compartment's size to the value determined by the formula in math. The overall units of the formula must be size/time, where the time units are the built-in units of time described in Section 4.4 and the size units are the units of size on the compartment (Section 4.7.5).

- In the case of a parameter, a RateRule sets the rate of change of the parameter's value to that determined by the formula in math. The overall units of the formula must be $x /$ time, where $x$ are the units of the parameter (Section 4.9.3).

In the context of a simulation, rate rules are in effect for simulation time $t>0$. Other types of rules and initial assignments are in effect at different times; Section 3.4.8 describes these conditions.

As mentioned in Section 4.11.3 for AssignmentRule, a model must not contain more than one RateRule or AssignmentRule object having the same value of variable; in other words, in the set of all assignment rules and rate rules in an SBML model, each variable appearing in the left-hand sides can only appear once. This simply follows from the fact that an indeterminate system would result if a model contained more than one assignment rule for the same variable or both an assignment rule and a rate rule for the same variable.

\subsubsection{Additional restrictions on rules}

An important design goal of SBML rule semantics is to ensure that a model's simulation and analysis results will not be dependent on when or how often rules are evaluated. To achieve this, SBML needs to place 
two additional restrictions on rule use in addition to the conditions described above regarding the use of AlgebraicRule, AssignmentRule and RateRule. The first concerns algebraic loops in the system of assignments in a model, and the second concerns overdetermined systems.

\section{The model must not contain algebraic loops}

The combined set of InitialAssignment, AssignmentRule and KineticLaw objects constitute a set of assignment statements that should be considered as a whole. (A KineticLaw object is counted as an assignment because it assigns a value to the symbol contained in the id attribute of the Reaction object in which it is defined.) This combined set of assignment statements must not contain algebraic loops - dependency chains between these statements must terminate. To put this more formally, consider a directed graph in which nodes are assignment statements and directed arcs exist for each occurrence of an SBML species, compartment or parameter symbol in an assignment statement's math element. Let the directed arcs point from the statement assigning the symbol to the statements that contain the symbol in their math element expressions. This graph must be acyclic.

SBML does not specify when or how often rules should be evaluated. Eliminating algebraic loops ensures that assignment statements can be evaluated any number of times without the result of those evaluations changing. As an example, consider the following equations:

$$
x=x+1, \quad y=z+200, \quad z=y+100
$$

If this set of equations were interpreted as a set of assignment statements, it would be invalid because the rule for $x$ refers to $x$ (exhibiting one type of loop), and the rule for $y$ refers to $z$ while the rule for $z$ refers back to $y$ (exhibiting another type of loop).

Conversely, the following set of equations would constitute a valid set of assignment statements:

$$
x=10, \quad y=z+200, \quad z=x+100
$$

\section{The model must not be overdetermined}

An SBML model must not be overdetermined; that is, a model must not define more equations than there are unknowns in a model. An SBML model that does not contain AlgebraicRule objects cannot be overdetermined.

Assessing whether a given continuous, deterministic, mathematical model is overdetermined does not require dynamic analysis; it can be done by analyzing the system of equations created from the model. One approach is to construct a bipartite graph in which one set of vertices represents the variables and the other the set of vertices represents the equations. Place edges between vertices such that variables in the system are linked to the equations that determine them. For algebraic equations, there will be edges between the equation and each variable occurring in the equation. For ordinary differential equations (such as those defined by rate rules or implied by the reaction rate definitions), there will be a single edge between the equation and the variable determined by that differential equation. A mathematical model is overdetermined if the maximal matchings (Chartrand, 1977) of the bipartite graph contain disconnected vertexes representing equations. (If one maximal matching has this property, then all the maximal matchings will have this property; i.e., it is only necessary to find one maximal matching.) Appendix D describes a method of applying this procedure to specific SBML data objects.

\subsubsection{Example of rule use}

This section contains an example set of rules. Consider the following set of equations:

$$
k=\frac{k_{3}}{k_{2}}, \quad s_{2}=\frac{k \cdot x}{1+k_{2}}, \quad A=0.10 \cdot x
$$

This can be encoded by the following scalar rule set (where the definitions of $\mathrm{x}, \mathrm{s}, \mathrm{k}, \mathrm{k} 2, \mathrm{k} 3$ and $\mathrm{A}$ are assumed to be located elsewhere in the model and not shown in this abbreviated example): 


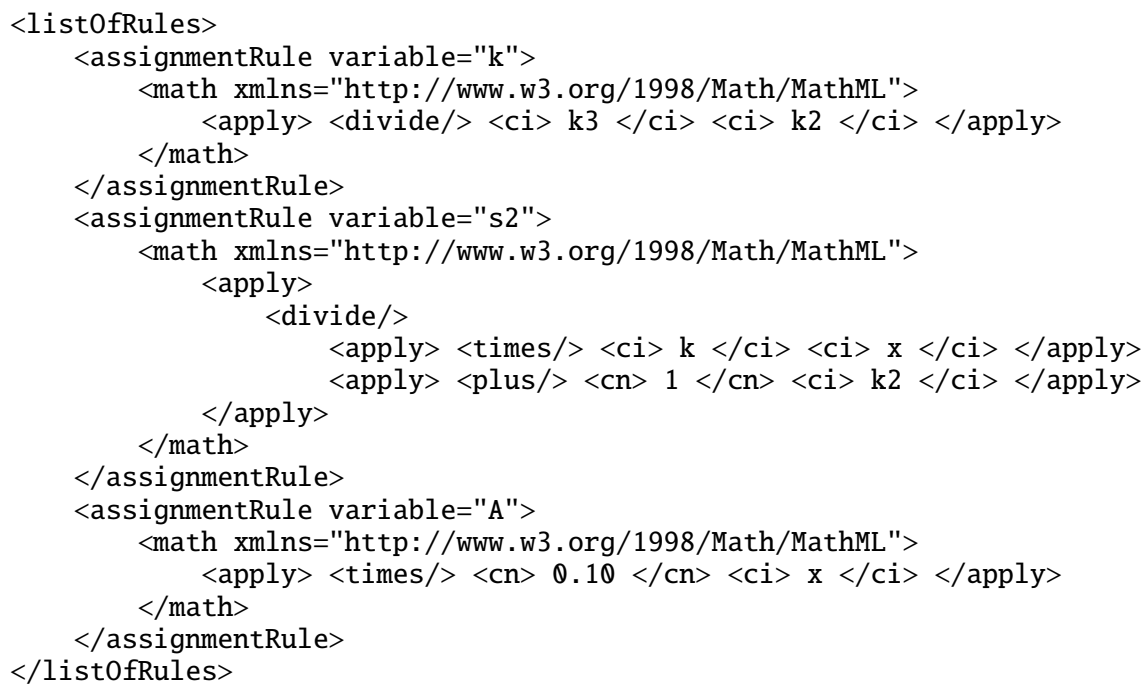

\subsection{Constraints}

The Constraint object is a mechanism for stating the assumptions under which a model is designed to operate. The constraints are statements about permissible values of different quantities in a model. Figure 20 shows the definition of the Constraint object class.

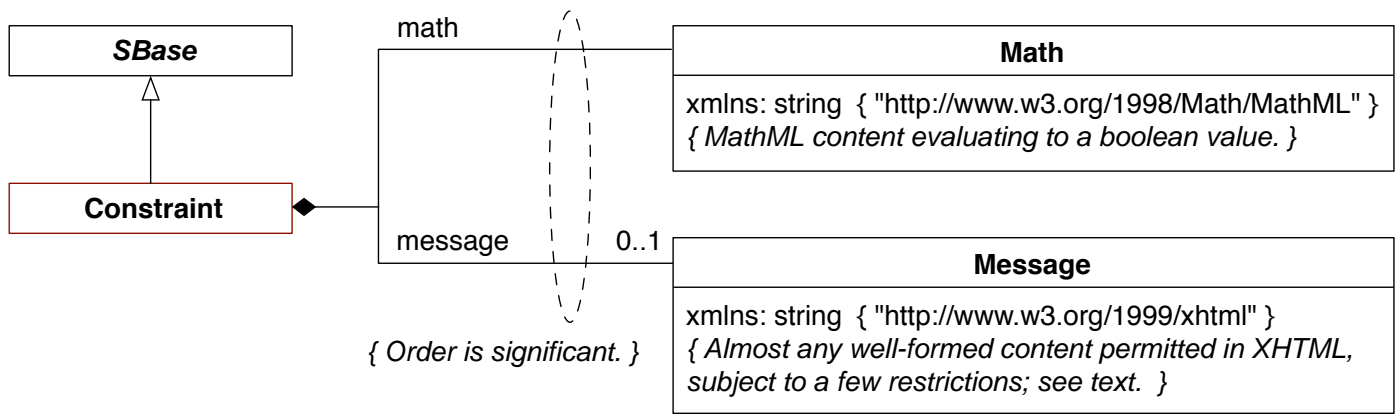

Figure 20: The definition of class Constraint. The contents of the Math class can be any MathML permitted in SBML, but it must return a boolean value. As shown above, an instance of Constraint can also contain zero or one instances of Message; this element is simply a wrapper (in the XML form, within $<$ message $>\quad \ldots \quad</$ message $>$ tags) for XHTML content. The same guidelines for XHTML content as explained in Section 3.2.3 for notes on Sbase also apply to the XHTML within messages in a Constraint. A sequence of one or more instances of Constraint objects can be located in an instance of ListOfConstraints in Model, as shown in Figure 10.

The essential meaning of a constraint is this: if a dynamical analysis of a model (such as a simulation) reaches a state in which a constraint is no longer satisfied, the results of the analysis are deemed invalid beginning with that point in time. The exact behavior of a software tool, upon encountering a constraint violation, is left up to the software; however, a software tool must somehow indicate to the user when a model's constraints are no longer satisfied. (Otherwise, a user may not realize that the analysis has reached an invalid state and is potentially producing nonsense results.) If a software tool does not have support for constraints, it should indicate this to the user when encountering a model containing constraints.

\subsubsection{The math element}

Constraint has one required subelement, math, containing a MathML formula defining the condition of the constraint. This formula must return a boolean value of "true" when the model is in a valid state. The formula can be an arbitrary expression referencing the variables and other entities in an SBML model. The evaluation of math and behavior of constraints are described in more detail in Section 4.12.4 below. 


\subsubsection{The message element}

A Constraint object has an optional element called message. This can contain a message in XHTML format that may be displayed to the user when the condition of the constraint in math evaluates to a value of "false". Software tools are not required to display the message, but it is recommended that they do so as a matter of best practice.

The XHTML content within a message element must follow the same restrictions as for the notes element on Sbase described in Section 3.2.3. For example, message must not contain an XML declaration or a DOCTYPE declaration, and the permitted content can only take one of the following general forms: (1) a complete XHTML document beginning with the element $<$ html $>$ and ending with $</$ html $>$; (2) the "body" portion of a document beginning with the element <body > and ending with </body>; or (3) XHTML content that is permitted within $\mathrm{a}<$ body $>\ldots<$ /body $>$ elements. Appendix F describes one approach to reading the message content.

\subsubsection{The sboTerm attribute}

The Constraint object inherits from Sbase the optional sboTerm attribute of type SBOTerm (see Sections 3.1.9 and 5). When a value is given to this attribute in a constraint definition, the value must be a valid SBO identifier referring to a mathematical expression (i.e., terms derived from SB0:0000064, "mathematical expression"). The Constraint should have an "is a" relationship with the SBO term, and the term should be the most precise (narrow) term that captures the role of the Constraint in the model.

As discussed in Section 5, SBO labels are optional information on a model. Applications are free to ignore sboTerm values. A model must be interpretable without the benefit of SBO labels.

\subsubsection{Semantics of constraints}

In the context of a simulation, a Constraint has effect at all times $t \geq 0$. Each Constraint's math element is first evaluated after any InitialAssignment definitions in a model at $t=0$ and can conceivably trigger at that point. (In other words, a simulation could fail a constraint immediately.)

Constraint definitions cannot and should not be used to compute the dynamical behavior of a model as part of, for example, simulation. Constraints may be used as input to non-dynamical analysis, for instance by expressing flux constraints for flux balance analysis.

The results of a simulation of a model containing a constraint are invalid from any simulation time at and after a point when the function given by the math returns a value of "false". Invalid simulation results do not make a prediction of the behavior of the biochemical reaction network represented by the model. The precise behavior of simulation tools is left undefined with respect to constraints. If invalid results are detected with respect to a given constraint, the message element (Section 4.12.2) may optionally be displayed to the user. The simulation tool may also halt the simulation or clearly delimit in output data the simulation time point at which the simulation results become invalid.

SBML does not impose restrictions on duplicate Constraint definitions or the order of evaluation of Constraint objects in a model. It is possible for a model to define multiple constraints all with the same math element. Since the failure of any constraint indicates that the model simulation has entered an invalid state, a system is not required to attempt to detect whether other constraints in the model have failed once any one constraint has failed.

\subsubsection{Example}

As an example, the following SBML fragment demonstrates the constraint that species $S_{1}$ should only have values between 1 and 100:

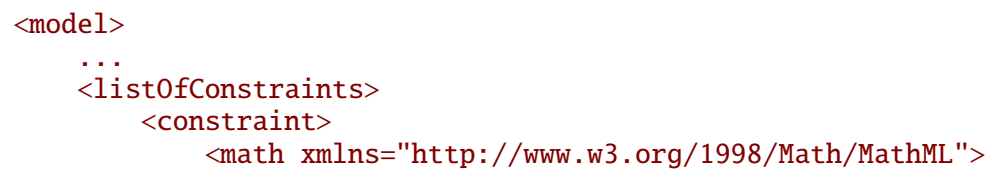




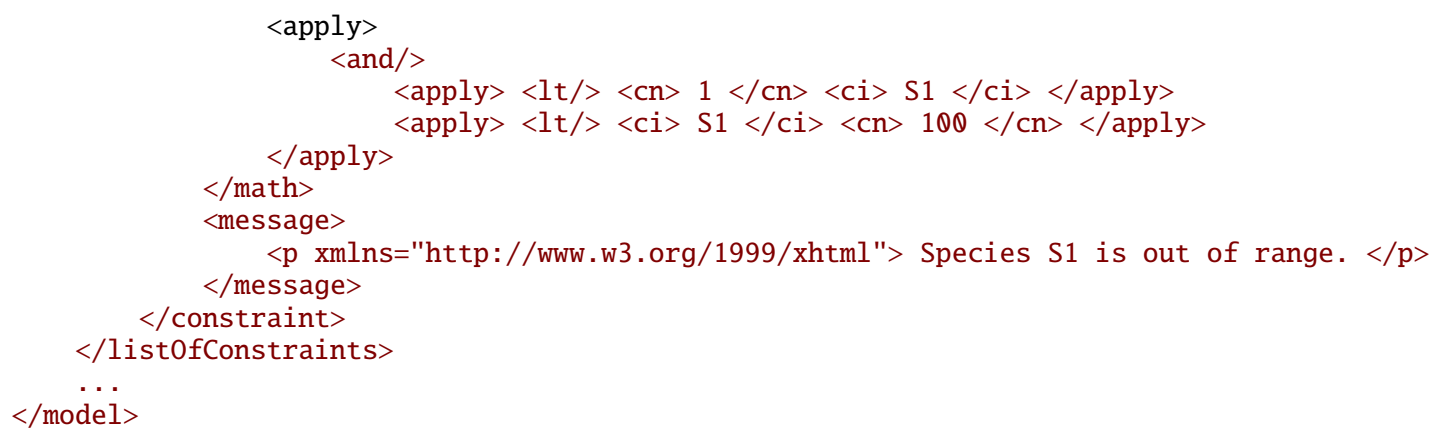

\subsection{Reactions}

A reaction represents any transformation, transport or binding process, typically a chemical reaction, that can change the quantity of one or more species. In SBML, a reaction is defined primarily in terms of the participating reactants and products (and their corresponding stoichiometries), along with optional modifier species, an optional rate at which the reaction takes place, and optional parameters. These various parts of a reaction are recorded in the SBML Reaction object class and other supporting data classes, defined in Figure 21 on the next page.

\subsubsection{Reaction}

Each reaction in an SBML model is defined using an instance of a Reaction object. As shown in Figure 21 on the following page, it contains several scalar attributes and several lists of objects.

\section{The id and name attributes}

As with most other main kinds of objects in SBML, the Reaction object class includes a mandatory attribute called id, of type SId, and an optional attribute name, of type string. The id attribute is used to give the reaction a unique identifier in the model. This identifier can be used in mathematical formulas elsewhere in an SBML model to represent the rate of that reaction; this usage is explained in detail in Section 4.13.7 below. The name attribute can be used to give the reaction a more free-form, descriptive name. The name and id attributes must be used as described in Section 3.3.

\section{The lists of reactants, products and modifiers}

The species participating as reactants, products, and/or modifiers in a reaction are declared using lists of SpeciesReference and/or ModifierSpeciesReference instances stored in list0fReactants, list0fProducts and list0fModifiers. SpeciesReference and ModifierSpeciesReference are described in more detail in Sections 4.13 .3 and 4.13 .4 below.

Certain restrictions are placed on the appearance of species in reaction definitions:

- The ability of a species to appear as a reactant or product of any reaction in a model is governed by certain flags in that species' definition; see Section 4.8.6 for more information.

- Any species appearing in the mathematical formula of the kineticLaw of a Reaction instance must be declared in at least one of that Reaction's lists of reactants, products, and/or modifiers. Put another way, it is an error for a reaction's kinetic law formula to refer to species that have not been declared for that reaction.

- A reaction definition can contain an empty list of reactants or an empty list of products, but it must have at least one reactant or product; in other words, a reaction without any reactant or product species is not permitted. (This restriction does not apply to modifier species, which remain optional in all cases.) 


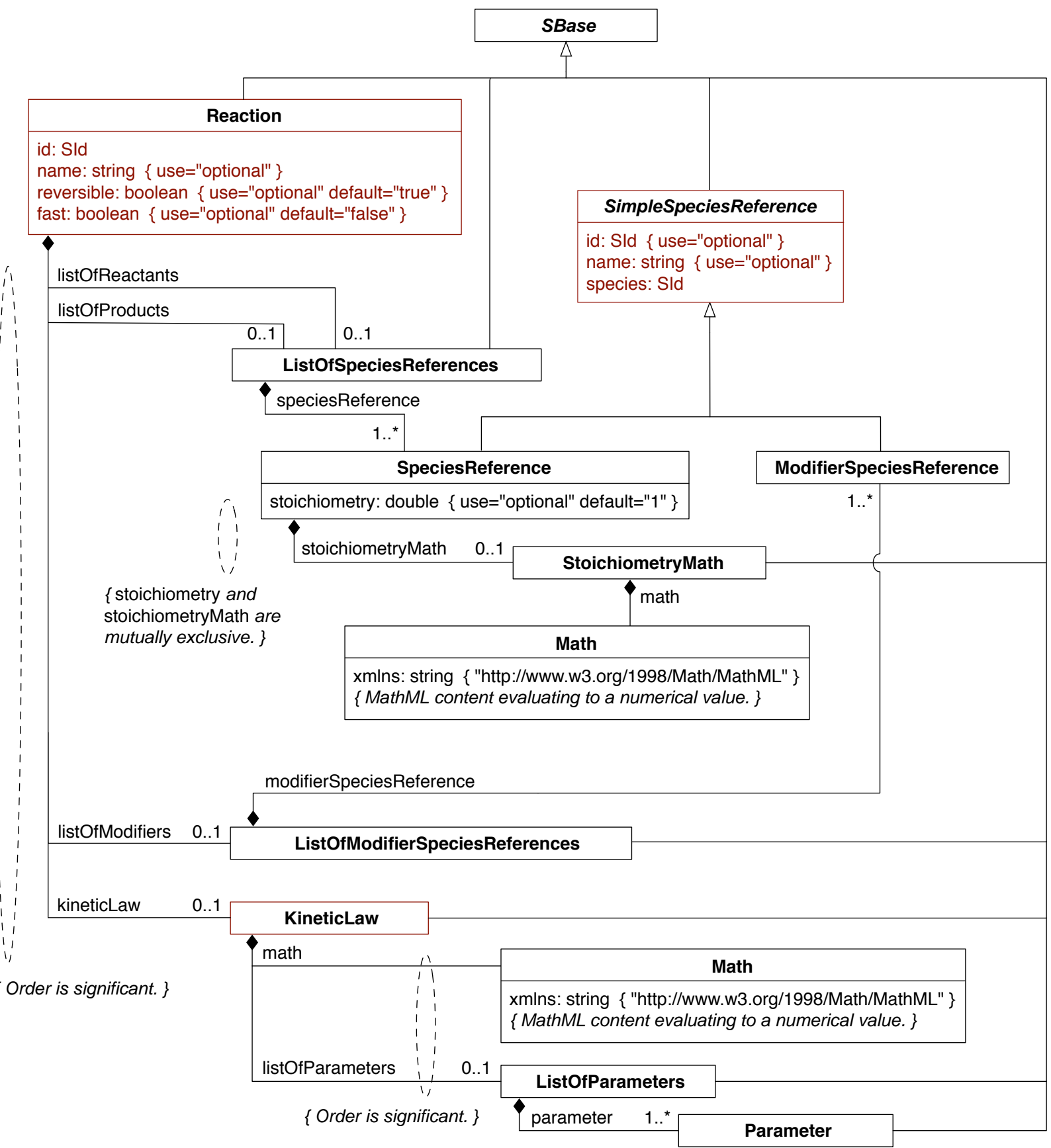

Figure 21: The definitions of classes Reaction, KineticLaw, SpeciesReference, ModifierSpeciesReference, as well as the container classes ListOfReactions, ListOfReactions, ListOfReactions, and ListOfParameters. Note that SimpleSpeciesReference is an abstract class used only to provide some common attributes to its derived classes. The class Parameter is defined in Section 4.9.

\section{The kineticLaw element}

A reaction can contain up to one KineticLaw object in the kineticLaw element of the Reaction. This "kinetic law" defines the speed at which the process defined by the reaction takes place. A detailed description of KineticLaw is left to Section 4.13.5 below. 
Note that the inclusion of a KineticLaw object in an instance of a Reaction component is optional; however, in general there is no useful default that can be substituted in place of a missing rate expression in a reaction. Moreover, a reaction's rate cannot be defined in any other way in SBML - InitialAssignment, AssignmentRule, RateRule, AlgebraicRule, Event, and other constructs in SBML cannot be used to set the reaction rate separately. Nevertheless, for some modeling applications, reactions without any defined rate can be perfectly acceptable.

\section{The reversible attribute}

The optional boolean attribute reversible indicates whether the reaction is reversible. The default is "true".

To say that a reaction is reversible is to say it can proceed in either the forward or the reverse direction. Although the reversibility of a reaction can sometimes be deduced by inspecting its rate expression, this is not always the case, especially for complicated expressions. Having a separate attribute supports the ability to perform some kinds of model analyses in the absence of performing a time-course simulation of the model. Moreover, the need in SBML to allow rate expressions (i.e., KineticLaw) to be optional leads to the need for a separate flag indicating reversibility. Information about reversibility in the absence of a KineticLaw in a Reaction is useful in certain kinds of structural analyses such as elementary mode analysis.

Mathematically, the reversible attribute on Reaction has no impact on the construction of the equations giving the overall rates of change of each species quantity in a model. A concrete explanation may help illustrate this. Suppose a model consists of multiple reactions, of which two particular irreversible reactions $R_{f}$ and $R_{r}$ are actually the forward and reverse processes of the same underlying reaction. The product species of $R_{f}$ necessarily will be the reactants of $R_{r}$, and the reactants of $R_{f}$ will be the products of $R_{r}$. Let $f_{f}(\mathbf{X})$ and $f_{r}(\mathbf{X})$ be the SBML kinetic rate formulas of $R_{f}$ and $R_{r}$, respectively, with $\mathbf{X}$ representing the species, parameters and compartments in the model. For the sake of this example, suppose we are using a continuous differential equation framework to simulate the system of reactions. Then for each species, we need to construct an expression representing the overall rate of change of that species' amount in the model. This overall expression will be a sum of the contributions of all the relevant rate formulas,

$$
\frac{d S}{d t}=\ldots-n \cdot f_{f}(\mathbf{X})+n \cdot f_{r}(\mathbf{X})+\ldots
$$

where $S$ is a reactant species of $R_{f}$ and a product of $R_{r}, n$ is the effective stoichiometry of $S$ in $R_{f}$ (which by implication must be the same as its stoichiometry in $R_{r}$ ), and "..." indicates other rate formulas in the model involving the particular species $S$. Now, contrast this to the case of an identical second SBML model, except that instead of having separate Reaction definitions for the forward and reverse reactions, this model has a single Reaction $R_{c}$ labeled as reversible and whose reactants and products are the same as those of $R_{f}$ in the first model. The rate of this reaction will be a formula $f_{c}=f_{f}(\mathbf{X})-f_{r}(\mathbf{X})$. In constructing an expression representing the overall rate of change for the species $S$ involved in that reaction, we will have

$$
\begin{aligned}
\frac{d S}{d t} & =\ldots-n \cdot f_{c}(\mathbf{X})+\ldots \\
& =\ldots-n \cdot f_{f}(\mathbf{X})+n \cdot f_{r}(\mathbf{X})+\ldots
\end{aligned}
$$

In other words, the result is the same final expression for the rate of change of a species. Although in this simple example we used an expression for $f_{c}$ that had clearly separated terms, in the general case the expression may have a more complicated form.

Note that labeling a reaction as irreversible is an assertion that the reaction always proceeds in the given forward direction. (Why else would it be flagged as irreversible?) This implies the rate expression in the KineticLaw always has a non-negative value during simulations. Software tools could provide a means of optionally testing that this condition holds. The presence of reversibility information in two places (i.e., the rate expression and the reversible flag) leaves open the possibility that a model could contain contradictory information, but the creation of such a model would be an error on the part of the software generating it. 


\section{The fast attribute}

The optional boolean attribute fast is another optional boolean attribute of Reaction. The attribute's default value is "false".

Previous definitions of SBML indicated that software tools could ignore this attribute if they did not implement support for the corresponding concept; however, further research has revealed that this is incorrect and fast cannot be ignored if it is set to "true". SBML Level 2 Version 3 therefore stipulates that if a model has any reactions with fast set to "true", a software tool must be able to respect the attribute or else indicate to the user that it does not have the capacity to do so. Analysis software cannot ignore the value of the fast attribute because doing so may lead to different results as compared to a software system that does make use of fast.

When a model contains true values for fast on any of its reactions, it is an indication that the creator of the model is distinguishing different time scales of reactions in the system. The model's reaction definitions are divided into two sets by the values of the fast attributes. The set of reactions having fast= "true" (known as fast reactions) should be assumed to be operating on a time scale significantly faster than the other reactions (the slow reactions). Fast reactions are considered to be instantaneous relative to the slow reactions.

Software tools must use a pseudo steady-state approximation for the set of fast reactions when constructing the system of equations for the model. More specifically, the set of reactions that have the fast attribute set to "true" forms a subsystem that should be described by a pseudo steady-state approximation in relationship to all other reactions in the model. Under this description, relaxation from any initial condition or perturbation from any intermediate state of this subsystem would be infinitely fast. Appendix E provides a technical explanation of an approach to solving systems with fast reactions.

The correctness of the approximation requires a significant separation of time scales between the fast reactions and other processes. This is not trivial to estimate a priori, and may even change over the course of a simulation, but can reasonably be assessed a posteriori in most cases.

\section{The sboTerm attribute on Reaction}

The Reaction object class inherits from Sbase the optional sboTerm attribute of type SBOTerm (see Sections 3.1.9 and 5). When a value is given to this attribute in a reaction definition, the value must be a valid SBO identifier referring to an event (i.e., terms derived from SB0:0000231, "event"). The Reaction object should have an "is a" relationship with the SBO term. The SBO term chosen should be the most precise (narrow) term that defines the event represented by the reaction as a whole. For example, a given reaction in a model might represent binding, which has a term in SBO (identifier SB0:0000177, definition "Interaction between several biochemical entities that results in the formation of a non-covalent complex"). The corresponding Reaction instance in SBML would have sboTerm="SBO:0000177".

As mentioned elsewhere, SBO labels are optional information on a model. Applications are free to ignore sboTerm values, and a model must be interpretable without the benefit of SBO labels. Section 5 gives more information about this principle and the use of SBO.

\subsubsection{The SimpleSpeciesReference abstract type}

As mentioned above, every species that enters into a given reaction must appear in that reaction's lists of reactants, products and/or modifiers. In an SBML model, all species that may participate in any reaction are listed in the list0fSpecies element of the top-level Model instance (see Section 4.2). Lists of products, reactants and modifiers in Reaction objects do not introduce new species, but rather, they refer back to those listed in the model's top-level list0fSpecies. For reactants and products, the connection is made using a SpeciesReference object; for modifiers, it is made using a ModifierSpeciesReference object. SimpleSpeciesReference, defined in Figure 21 on page 64, is an abstract type that serves as the parent class of both SpeciesReference and ModifierSpeciesReference. It is used simply to hold the attributes and elements that are common to the latter two objects. 


\section{The id and name attributes}

The optional identifier stored in the id attribute allows SpeciesReference and ModifierSpeciesReference instances to be referenced from other object. No SBML object classes currently do this; however, such classes are anticipated in future SBML Levels. The value of id must be a text string conforming to the syntax permitted by the SId data type described in Section 3.1.7. The id value (whether it is in a SpeciesReference or ModifierSpeciesReference object) exists in the global namespace of the model, as described in Section 3.3. The id and name attributes must be used as described in Section 3.3.

\section{The species attribute}

The SimpleSpeciesReference object class has a required attribute, species, of type SId. As with the other attributes, it is inherited by SpeciesReference and ModifierSpeciesReference. The value of species must be the identifier of a species defined in the enclosing Model. The species is thereby declared as participating in the reaction being defined. The precise role of that species as a reactant, product, or modifier in the reaction is determined by the subtype of SimpleSpeciesReference (i.e., either SpeciesReference or ModifierSpeciesReference) in which the identifier appears.

\section{The sboTerm attribute}

The class SimpleSpeciesReference inherits from Sbase the optional sboTerm attribute of type SBOTerm (see Sections 3.1.9 and 5). This means that the object classes derived from SimpleSpeciesReference, namely SpeciesReference and ModifierSpeciesReference, all have sboTerm attributes. When a value is given to this attribute, it must be a valid SBO identifier referring to a participant role. The appropriate SBO term depends on whether the object is a reactant, product or modifier. If a reactant, then it should be a term in the SB0:0000010, "reactant" hierarchy; if a product, then it should be a term in the SB0:0000011, "product" hierarchy; and if a modifier, then it should be a term in the SB0:0000019, "modifier" hierarchy. The SpeciesReference and ModifierSpeciesReference instances should have an "is a" relationship to the term identified by the SBO identifier.

The SBO terms chosen should be the most precise (narrow) one that defines the role of the species in the reaction. An SBO reactant term can only be assigned to the sboTerm attribute of a SpeciesReference instance when that instance is contained in the list of reactants in the containing Reaction instance. Similarly, an SBO product term can only be assigned to the sboTerm attribute of a SpeciesReference instance when that instance is contained in the list of products in the containing Reaction instance.

\subsubsection{SpeciesReference}

The Reaction object class provides a way to express which species act as reactants and which species act as products in a reaction. In a given reaction, references to those species acting as reactants and/or products are made using instances of SpeciesReference objects in Reaction's lists of reactants and products. The SpeciesReference structure inherits the mandatory attribute species and optional attributes id, name, and sboTerm, from the parent type SimpleSpeciesReference; see Section 4.13.2 for their definitions. It also defines attribute stoichiometry and element stoichiometryMath, described below.

The species attribute value must be the identifier of an existing species defined in the enclosing Model; the species is thereby designated as a reactant or product in the reaction. Which one it is (i.e., reactant or product) is indicated by whether the SpeciesReference appears in the Reaction's reactant or product lists.

\section{The stoichiometry attribute and stoichiometryMath element}

Product and reactant stoichiometries can be specified using either stoichiometry or stoichiometryMath in a SpeciesReference object. The stoichiometry attribute is of type double and should contain values greater than zero (0). The stoichiometryMath element is implemented as an element containing a MathML expression. These two are mutually exclusive; only one of stoichiometry or stoichiometryMath should be defined in a given SpeciesReference instance. When neither the attribute nor the element is present, the value of stoichiometry in the SpeciesReference instance defaults to " 1 ". 
For maximum interoperability, SpeciesReference's stoichiometry attribute should be used in preference to stoichiometryMath when a species' stoichiometry is a simple scalar number (integer or decimal). When the stoichiometry is a rational number, or when it is a more complicated formula, stoichiometryMath must be used. The MathML expression in stoichiometryMath may also refer to identifiers of entities in a model (except reaction identifiers), as discussed in Section 3.4.3. However, the only species identifiers that can be used in stoichiometryMath are those referenced in the Reaction list of reactants, products and modifiers.

The stoichiometry attribute and the stoichiometryMath element, when either is used, is each interpreted as a factor applied to the reaction rate to give the rate of change of the species identified by the species attribute. This is the normal interpretation of a stoichiometry, but in SBML, one additional consideration has to be taken into account. The reaction rate, which is the result of the KineticLaw math element, is always in the model's substance per time units. However, the rate of change of the species will involve the species' substance units (i.e., the units identified by the Species object's substanceUnits attribute), and these units may be different from the model's default substance units. If the units are different, the stoichiometry must incorporate a conversion factor for converting the model's substance units to the species' substance units. The conversion factor is assumed to be included in the scalar value of the stoichiometry attribute if stoichiometry is used. If instead stoichiometryMath is used, then the product of the model's substance units times the stoichiometryMath units must match the substance units of the species. Note that in either case, if the species' units and the model's default substance units are the same, the stoichiometry ends up being a dimensionless number and equivalent to the standard chemical stoichiometry found in textbooks. Examples and more explanations of this are given in Section 4.13.6.

The following is a simple example of a species reference for species " $\mathrm{X} 0$ ", with stoichiometry " 2 ", in a list of reactants within a reaction having the identifier "J1":

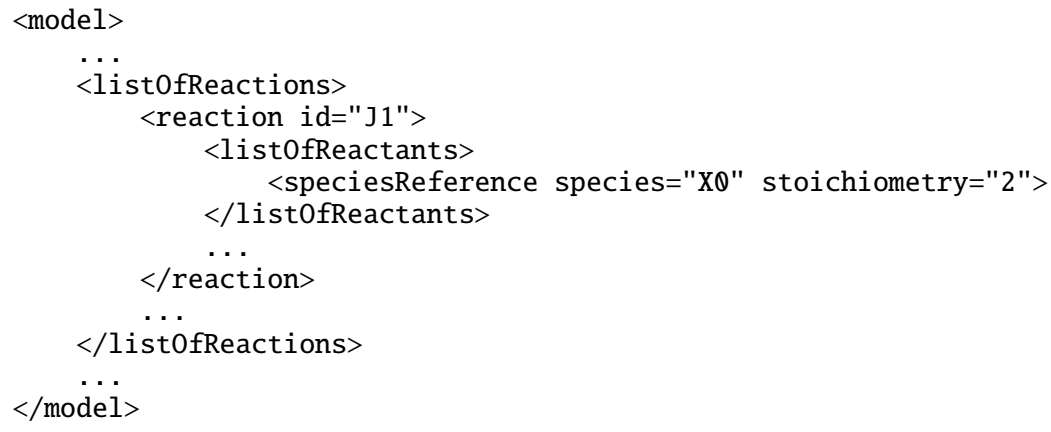

The following is a more complex example of a species reference for species "X0", with a stoichiometry formula consisting of a rational number:

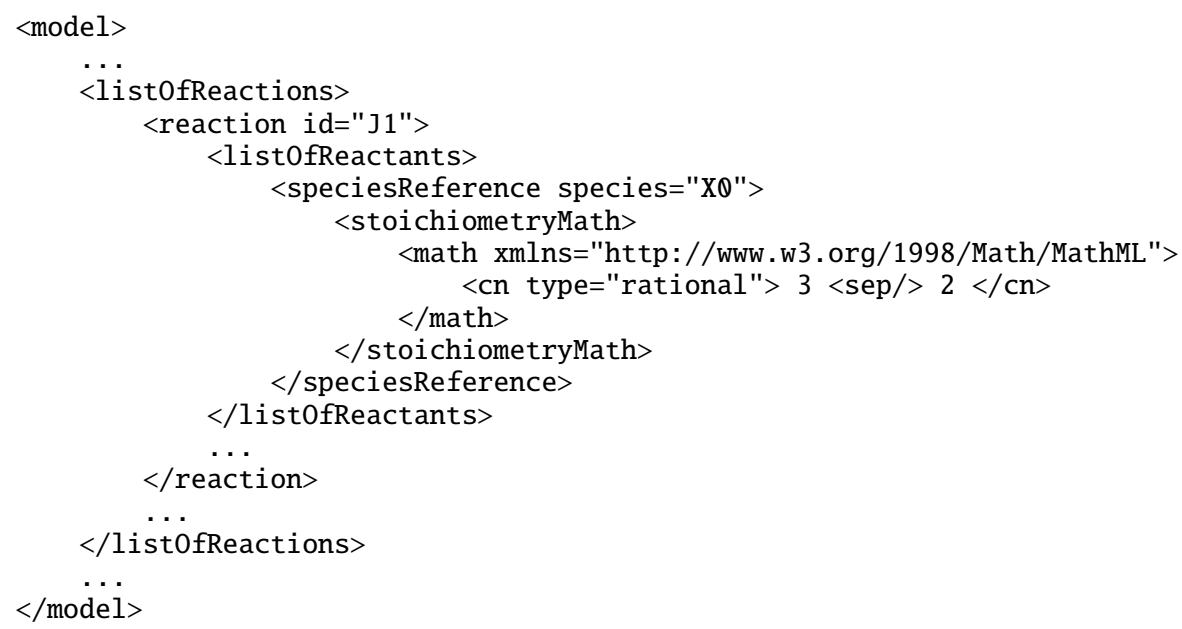

A species can occur more than once in the lists of reactants and products of a given Reaction instance. The effective stoichiometry for a species in a reaction is the sum of the stoichiometry values given in the 
SpeciesReference objects in the list of products minus the sum of stoichiometry values given in the SpeciesReference objects in the list of reactants. A positive value indicates the species is effectively a product and a negative value indicates the species is effectively a reactant. SBML places no restrictions on the effective stoichiometry of a species in a reaction; for example, it can be zero. In the following SBML fragment, the two reactions have the same effective stoichiometry for all their species:

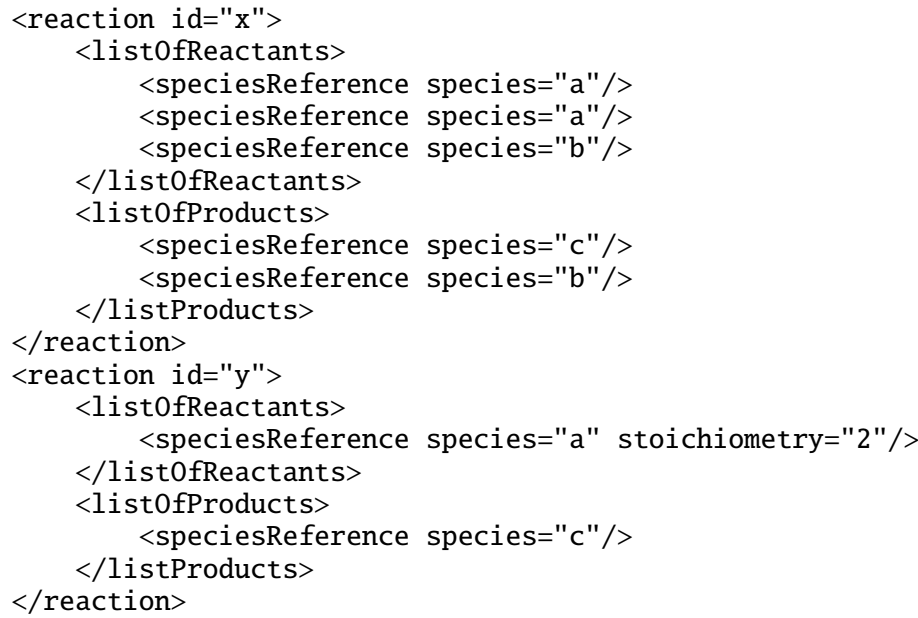

\subsubsection{ModifierSpeciesReference}

Sometimes a species appears in the kinetic rate formula of a reaction but is itself neither created nor destroyed in that reaction (for example, because it acts as a catalyst or inhibitor). In SBML, all such species are simply called modifiers without regard to the detailed role of those species in the model. The Reaction object class provides a way to express which species act as modifiers in a given reaction. This is the purpose of the list of modifiers available in Reaction. The list contains instances of ModifierSpeciesReference object.

As shown in Figure 21 on page 64, the ModifierSpeciesReference class inherits the mandatory attribute species and optional attributes id and name from the parent class SimpleSpeciesReference; see Section 4.13.2 for their precise definitions.

The value of the species attribute must be the identifier of a species defined in the enclosing Model; this species is designated as a modifier for the current reaction. A reaction may have any number of modifiers. It is permissible for a modifier species to appear simultaneously in the list of reactants and products of the same reaction where it is designated as a modifier, as well as to appear in the list of reactants, products and modifiers of other reactions in the model.

\subsubsection{KineticLaw}

The KineticLaw object class is used to describe the rate at which the process defined by the Reaction takes place. As shown in Figure 21 on page 64, KineticLaw has elements called math and list0fParameters, in addition to the attributes and elements it inherits from Sbase.

Previous definitions of SBML included two additional attributes called substanceUnits and timeUnits, which allowed the substance/time units of the reaction rate expression to be defined on a per-reaction basis. SBML Level 2 Version 2 and Version 3 removed these attributes for several reasons. First, the introduction in SBML Level 2 Version 2 of mass and dimensionless units as possible units of substance, coupled with the previous facility for defining the units of each reaction separately and the ability to use non-integer stoichiometries, lead to the possibility of creating a valid model whose reactions nevertheless could not be integrated into a system of equations without outside knowledge for converting the quantities used. (As a simple example, consider if one reaction is defined to be in grams per second and another in moles per second, and species are given in moles: converting from mass to moles would require knowing the molecular mass of the species.) Second, the ability to change the units of a reaction provided the potential of creating unintuitive and difficult-to-reconcile systems of equations, yet the feature added little functionality to SBML. 
The absence of substanceUnits does not prevent the definition of any reactions; it only results in requiring the generator of the model to be explicit about any necessary conversion factors. Third, few if any software tools have ever correctly implemented support for substanceUnits, which made the use of this attribute in a model an impediment to interoperability. Fourth, examination of real-life models revealed that a frequent reason for using substanceUnits was to set the units of all reactions to the same set of substance units, which is better achieved by setting the model-wide values of "substance".

\section{The math element}

As shown in Figure 21 on page 64, KineticLaw has a element called math for holding a MathML formula defining the rate of the reaction. The expression in math may refer to species identifiers, as discussed in Section 3.4.3. The only Species identifiers that can be used in math are those declared in the lists of reactants, products and modifiers in the Reaction object (see Sections 4.13.2, 4.13.3 and 4.13.4). Parameter identifiers may be taken from the KineticLaw's list of local parameters (see below) or the parameters defined globally on the Model instance.

Section 4.13.6 provides important discussions about the meaning and interpretation of SBML "kinetic laws".

\section{The list of parameters}

An instance of KineticLaw can contain a list of one or more Parameter objects (Section 4.9) which define new parameters whose identifiers can be used in the math formula. As discussed in Section 3.3.1, reactions introduce local namespaces for parameter identifiers, and within a KineticLaw object, a local parameter whose identifier is identical to a global parameter defined in the model takes precedence over that global parameter.

The type of object used to define a parameter inside KineticLaw is the same Parameter object class used to define global parameters (Section 4.9). This simplifies the SBML language and reduces the number of unique types of data objects. However, there is a difference between local and global parameters: in the case of parameters defined locally to a KineticLaw, there is no means by which the parameter values can be changed. Consequently, such parameters' values are always constant, and the constant attribute in their definitions must always have a value of "true" (either explicitly or left to its default value).

\section{The sboTerm attribute}

KineticLaw inherits from Sbase the optional attribute called sboTerm of type SBOTerm (see Section 5). When a value is given to this attribute, the value must be an SBO identifier referring to a term from the SB0:0000001, "rate law" vocabulary defined in SBO. The relationship is of the form "the kinetic law is a X", where X is the SBO term. The SBO term chosen should be the most precise (narrow) term that defines the type of reaction rate expression encoded by the KineticLaw instance.

As explained in Section 4.2.2, there is an interaction between the SBO terms chosen for a model's KineticLaw instances and the sboTerm value given to the overall Model instance. The interaction is as follows. In SBO, each definition of a rate expression itself includes its own SBO reference to a mathematical framework. When a given KineticLaw element refers to a rate law defined in SBO, it therefore simultaneously also identifies a framework. If a Model instance has a value for sboTerm, it implies that all reaction kinetics in the model assume the same mathematical framework. In that case, the frameworks referenced in the SBO definitions of individual reaction rate expressions may be ignored. Consequently, if the mathematical frameworks underlying the definitions of different reactions in a model should be observed individually (e.g., because the model mixes frameworks), then there should not be a value assigned to sboTerm on the enclosing Model.

\section{Example}

The following is an example of a Reaction object that defines a reaction with identifier $J_{1}$, in which $X_{0} \rightarrow S_{1}$ at a rate given by $k \cdot\left[X_{0}\right] \cdot\left[S_{2}\right]$, where $S_{2}$ is a catalyst and $k$ is a parameter, and the square brackets symbolizes that the species quantities have units of concentration. The example demonstrates the use of species references and KineticLaw objects. The units on the species here are the defaults of substance/volume (see Section 4.8), and so the rate expression $k \cdot\left[X_{0}\right] \cdot\left[S_{2}\right]$ needs to be multiplied by the compartment volume (represented by its identifier, "c1") to produce the final units of substance/time for the rate expression. 


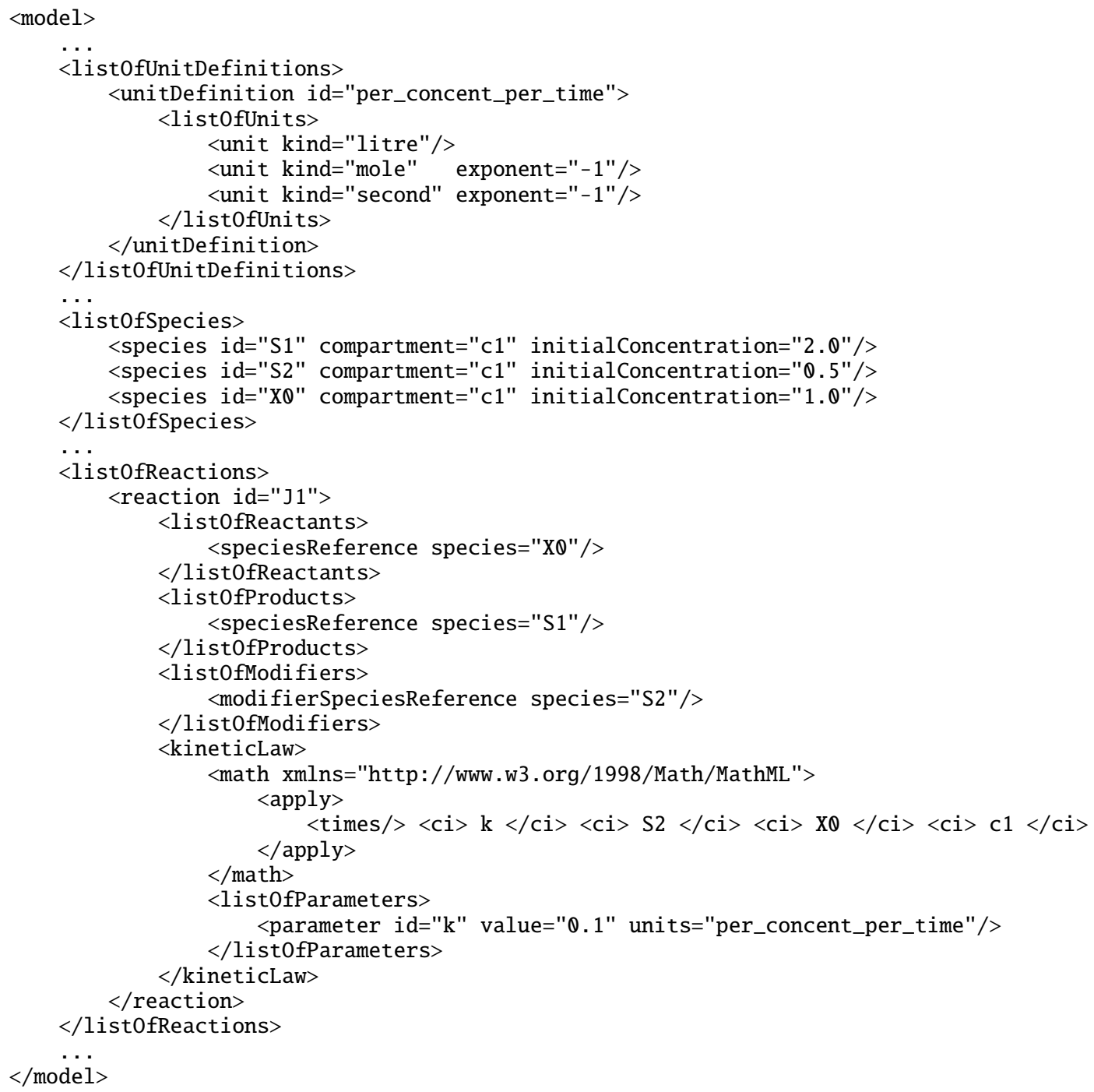

\subsubsection{Traditional rate laws versus SBML "kinetic laws"}

It is important to make clear that a "kinetic law" in SBML is not identical to a traditional rate law. The reason is that SBML must support multicompartment models, and the units normally used in traditional rate laws as well as some conventional single-compartment modeling packages are problematic when used for defining reactions between multiple compartments.

When modeling species as continuous amounts (e.g., concentrations), the rate laws used are traditionally expressed in terms of amount of substance concentration per time, embodying a tacit assumption that reactants and products are all located in a single, constant volume. Attempting to describe reactions between multiple volumes using concentration/time (which is to say, substance/volume/time) quickly leads to difficulties. Here is an illustration of this. Suppose we have two species pools $S_{1}$ and $S_{2}$, with $S_{1}$ located in a compartment having volume $V_{1}$, and $S_{2}$ located in a compartment having volume $V_{2}$. Let the volume $V_{2}=3 V_{1}$. Now consider a transport reaction $S_{1} \rightarrow S_{2}$ in which the species $S_{1}$ is moved from the first compartment to the second. Assume the simplest type of chemical kinetics, in which the rate of the transport reaction is controlled by the activity of $S_{1}$ and this rate is equal to some constant $k$ times the activity of $S_{1}$. For the sake of simplicity, assume $S_{1}$ is in a diluted solution and thus that the activity of $S_{1}$ can be taken to be equal to its concentration $\left[S_{1}\right]$. The rate expression will therefore be $k \cdot\left[S_{1}\right]$, with the units of $k$ being 1/time. Then: 


$$
\frac{d\left[S_{2}\right]}{d t}=-\frac{d\left[S_{1}\right]}{d t}=k \cdot\left[S_{1}\right]
$$

So far, this looks normal - until we consider the number of molecules of $S_{1}$ that disappear from the compartment of volume $V_{1}$ and appear in the compartment of volume $V_{2}$. The number of molecules of $S_{1}$ (call this $n_{S_{1}}$ ) is given by $\left[S_{1}\right] \cdot V_{1}$ and the number of molecules of $S_{2}$ (call this $n_{S_{2}}$ ) is given by $\left[S_{2}\right] \cdot V_{2}$. Since our volumes have the relationship $V_{2} / V_{1}=3$, the relationship above implies that $n_{S_{1}}=k \cdot\left[S_{1}\right] \cdot V_{1}$ molecules disappear from the first compartment per unit of time and $n_{S_{2}}=3 \cdot k \cdot\left[S_{1}\right] \cdot V_{1}$ molecules appear in the second compartment. In other words, we have created matter out of nothing!

The problem lies in the use of concentrations as the measure of what is transfered by the reaction, because concentrations depend on volumes and the scenario involves multiple unequal volumes. The problem is not limited to using concentrations or volumes; the same problem also exists when using density, i.e., mass/volume, and dependency on other spatial distributions (i.e., areas or lengths). What must be done instead is to consider the number of "items" being acted upon by a reaction process irrespective of their distribution in space (volume, area or length). An "item" in this context may be a molecule, particle, mass, or other "thing", as long as the substance measurement is independent of the size of the space in which the items are located.

For the current example, the expressions in terms of $n_{S_{1}}$ and $n_{S_{2}}$ are straightforward:

$$
\frac{d n_{S_{2}}}{d t}=-\frac{d n_{S_{1}}}{d t}=k \cdot\left[S_{1}\right] \cdot V_{1}
$$

Given numbers of items, it is then easy to recover concentrations by dividing the item counts of each species by the volume of the compartment in which the species is located: $\left[S_{1}\right]=n_{S_{1}} / V_{1}$ and $\left[S_{2}\right]=n_{S_{2}} / V_{2}$.

The need to support multicompartment models requires that the reaction rates in SBML to be expressed in terms of substance/time, rather than the more typical substance/size/time. As a result, modelers and software tools in general cannot insert textbook rate laws unmodified as the rate expression in the math element of a KineticLaw. The unusual term "kinetic law" was chosen to alert users to this difference. We explain the general principles of converting rate laws in the following paragraphs.

\section{Basic cases}

Let us expand the simple example above by adding a second reaction, to create the system

$$
S_{1} \rightarrow S_{2} \rightarrow S_{3}
$$

with the left-hand reaction's rate (call this $r_{1}$ ) being given as $k_{1} \cdot\left[S_{1}\right]$ and the rate of the right-hand reaction (call it $r_{2}$ ) as $k_{2} \cdot\left[S_{2}\right]$. Also assume each species is located in a different compartment:

$$
\begin{array}{lll}
S_{1} & \text { located in compartment } C_{1} & \text { with volume } V_{1} \\
S_{2} & \text { located in compartment } C_{2} & \text { with volume } V_{2} \\
S_{3} & \text { located in compartment } C_{3} & \text { with volume } V_{3}
\end{array}
$$

As before, converting the rate of the first reaction $\left(S_{1} \rightarrow S_{2}\right)$ to units of substance/time in this case is a simple matter of multiplying by the volume of the compartment where the reactants are located, leading to the following SBML rate formula:

$$
R_{1}=r_{1} \cdot V_{1}=-k_{1} \cdot\left[S_{1}\right] \cdot V_{1}
$$

The second rate expression becomes

$$
R_{2}=r_{2} \cdot V_{2}=-k_{2} \cdot\left[S_{2}\right] \cdot V_{2}
$$

The units of $k_{1}$ and $k_{2}$ are $1 /$ time (often $1 /$ sec, but not necessarily), as is typical for reactions that are firstorder in one reactant. The expressions $R_{1}$ and $R_{2}$ are what would be written in KineticLaw math definitions for the two reactions in this system. The formulas give the speed of each reaction in terms of the substance change over time. The reader of the SBML model needs to combine the individual contributions of each 
reaction to construct equations for the overall rates of change of each species in the model using these expressions. In terms of differential equations, these are:

$$
\begin{array}{ll}
\frac{d n_{S_{1}}}{d t}=-R_{1} & =-k_{1} \cdot\left[S_{1}\right] \cdot V_{1} \\
\frac{d n_{S_{2}}}{d t}=+R_{1}-R_{2} & =+k_{1} \cdot\left[S_{1}\right] \cdot V_{1}-k_{2} \cdot\left[S_{2}\right] \cdot V_{2} \\
\frac{d n_{S_{3}}}{d t}=\quad+R_{2}= & +k_{2} \cdot\left[S_{2}\right] \cdot V_{2}
\end{array}
$$

To recover the concentration values, we add the following to the system of equations:

$$
\begin{aligned}
& {\left[S_{1}\right]=n_{S_{1}} / V_{1}} \\
& {\left[S_{2}\right]=n_{S_{2}} / V_{2}} \\
& {\left[S_{3}\right]=n_{S_{3}} / V_{3}}
\end{aligned}
$$

Note that this formulation works properly even if the compartment sizes $V_{1}, V_{2}$ and $V_{3}$ vary during simulation.

Extrapolating from this example, we can now provide a general approach to translating a system of reactions involving species located in multiple compartments, for the restricted case where all reactants of any given reaction are in the same compartment (but where the compartments involved may be different for each reaction). For a species $S_{i}$ involved in $m$ reactions whose rates are given (in "textbook" form, without volume adjustments) as $r_{1}, r_{2}, \ldots, r_{m}$, where the reactants of $r_{j}$ are located in the compartment of size $V_{j}$,

$$
\begin{aligned}
\frac{d n_{S_{i}}}{d t}= & \operatorname{sign}_{1} \cdot \operatorname{stoich}_{1} \cdot r_{1} \cdot V_{1} \\
& +\operatorname{sign}_{2} \cdot \operatorname{stoich}_{2} \cdot r_{2} \cdot V_{2} \\
& +\ldots \\
& +\operatorname{sign}_{m} \cdot \operatorname{stoich}_{m} \cdot r_{m} \cdot V_{m} \\
{\left[S_{i}\right]=} & n_{S_{i}} / V_{i}
\end{aligned}
$$

In Equation (7), each term $\operatorname{sign}_{j}$ is "-" if $S_{i}$ is a reactant in $r_{j}$ and "+" if it is a product, and each term stoich ${ }_{j}$ is the stoichiometry of $S_{i}$ in reaction $r_{j}$. Letting $R_{j}=r_{j} \cdot V_{j}$ represent the form of the rate expressions as they would be written in the KineticLaw math elements, then we can equivalently write

$$
\begin{aligned}
\frac{d n_{S_{i}}}{d t}= & \operatorname{sign}_{1} \cdot \text { stoich }_{1} \cdot R_{1} \\
& +\operatorname{sign}_{2} \cdot \operatorname{stoich}_{2} \cdot R_{2} \\
& +\ldots \\
& +\operatorname{sign}_{m} \cdot \operatorname{stoich}_{m} \cdot R_{m} \\
{\left[S_{i}\right]=} & n_{S_{i}} / V_{i}
\end{aligned}
$$

This approach preserves the use of concentration terms within the reaction rate expressions so that the core of those rate expressions can be ordinary rate laws. This is important when modeling species as continuous quantities, because most textbook rate expressions are measured in terms of concentrations, and most rate constants have units involving concentration rather than item counts. For example, the second-order rate constant in a mass-action rate law has units of $1 /(M \cdot s)$, which is to say, volume/(substance-time); this constant is then multiplied by two concentration terms. Reaction definitions in SBML models can be constructed by taking such expressions and multiplying them by the volume of the compartment in which the reactants are located. By contrast, if we were to simply replace concentrations of species by item counts in such rate laws, it would in most cases be incorrect. At the very least, the constants in the equations would need to be converted in some way to make such expressions valid. 
The preceding discussion of problems involving rate laws concerns modeling approaches that use continuous quantities. There is an alternative approach to modeling that instead treats species as discrete populations (Wilkinson, 2006). In those cases, the rate expressions already use substance or item counts rather than concentrations and there is no need to convert them.

A full SBML example of translating a complete multicompartmental model into ODEs is given in Section 7.7. An example of translating a discrete model is given in Section 7.3.

\section{Advanced cases}

The explanation above applies to reactions where all of the reactants are in the same compartment. What about cases where two or more reactant species are in separate compartments?

This is a more difficult situation, and the guidelines described above for Equation (7) cannot always be applied because there will be more than one compartment size term by which the core rate expression needs to be multiplied. Unfortunately, there is often no straightforward way to mechanically convert such models without requiring a more significant change to the reaction rate expression. An example will help illustrate the difficulty. Suppose we have a simple reaction system consisting of only

$$
S_{1}+S_{2} \rightarrow S_{3}
$$

where $S_{1}, S_{2}$ and $S_{3}$ are each located in separate compartments with volumes $V_{1}, V_{2}$ and $V_{3}$, and the rate expression is given as $k \cdot\left[S_{1}\right] \cdot\left[S_{2}\right]$. (In reality, one would not use such a rate law in this case, but for the sake of this example, let us ignore the fact that a mass-action rate law would actually involve an assumption that all reactants are in a well-mixed solution.) A straightforward examination of the possibilities eventually leads to the conclusion that in order to take account of the multiple volumes, the rate expressions in terms of substance/time have to be written as

$$
\frac{d n_{S_{i}}}{d t}=-k^{\prime}\left(V_{1}, V_{2}\right) \cdot\left(\left[S_{1}\right] \cdot V_{1}\right) \cdot\left(\left[S_{2}\right] \cdot V_{2}\right)
$$

The crux of the problem is that the new factor $k^{\prime}\left(V_{1}, V_{2}\right)$ is not the original $k$; to make the overall units of the expression work out, $k^{\prime}\left(V_{1}, V_{2}\right)$ must be a function of the volumes, and its value must change if $V_{1}$ or $V_{2}$ changes. It is no longer a standard rate constant. In an SBML model, it is easy to define an AssignmentRule to compute the value of $k^{\prime}$ based on $k, V_{1}, V_{2}$, and possibly other variables in the system as needed, but only the modeler can determine the proper formula for their particular modeling situation. (For example, the modeler may know that in their hypothesized physical system, the reaction actually takes place completely in one or the other compartment and therefore the factor should be designed accordingly, or perhaps the reaction takes place on a membrane between compartments and a scaling factor based on the area should be used.)

Thus, although these models can be represented in SBML, constructing the correct rate expression in terms of substance/time units depends on problem-specific knowledge, and we cannot provide a general recipe.

\section{Mixed species units}

The discussion so far has assumed that all of the species appearing in a given reaction's rate expression had the same units, whether they be concentration or amounts or other. However, Species objects can each declare units separately. What happens then?

It is important to realize that implicit conversions in this situation are not defined in SBML. A species identifier appearing in a mathematical expression has the units attributed to that species (see Section 4.8.5 for a definition of the the units of the species). If a reaction contains species each having different units, the creator of the SBML model must explicitly incorporate the necessary conversions to make the units of the rate expression consistent. The most appropriate way is to include the conversion factor as part of the value of stoichiometry or stoichiometryMath in the SpeciesReference for that species. 
An example may help illustrate this. Suppose we have a system of two biochemical reactions with mass-action kinetics, written in the traditional form

$$
\begin{aligned}
A+2 B \stackrel{k_{1}}{\longrightarrow} C \\
C \stackrel{k_{2}}{\longrightarrow} 3 A
\end{aligned}
$$

with the units of $k_{1}$ being $l i t e r^{2} s^{2} m o l^{-2}$ and $k_{2}$ 's units being $s^{-1}$. Assume the reactions take place in a single compartment of volume $V$, but now let us throw a wrench into the problem: suppose that the species in the model are defined with mixed units as follows:

$$
\begin{array}{ll}
A & \text { is in millimoles per litre } \\
B & \text { is in grams per litre } \\
C & \text { is in items per litre }
\end{array}
$$

When biochemical reaction equations of the form (9) above are written, the units of species' quantities usually are assumed to be the same, and therefore the stoichiometries in the reaction equations (9) represent simple ratios between the quantities of the species in those units. "One mole of this and two moles of that react to produce one mole of that other.") If instead the quantities of the species are given in mixed units, as in the present example, the quantities not only need to be in proper stoichiometric relationships, the units also have to be made consistent. In SBML, this is done by appropriately setting the stoichiometry attribute value in the species references of the lists of reactants, products and modifiers in a Reaction. This then permits a properly balanced system of equations to be constructed for each species' rate of change of quantity.

In the present example, the SBML "kinetic law" formulas for the reaction rates will be written as conventional mass-action reaction rates adjusted for volume as described previously,

$$
\begin{aligned}
& R_{1}=k_{1}{ }^{\prime} \cdot[A] \cdot[B]^{2} \cdot V \\
& R_{2}=k_{2}{ }^{\prime} \cdot[C] \cdot V
\end{aligned}
$$

where $k_{2}{ }^{\prime}=k_{2}$ for this particular example but $k_{1}{ }^{\prime}$ will depend on other units as described in the paragraphs below. When these formulas are combined into overall expressions for the rates of change of $A, B$, and $C$, the result is

$$
\begin{aligned}
& d A / d t=-a_{1} \cdot R_{1}+a_{2} \cdot R_{2} \\
& d B / d t=-b_{1} \cdot R_{1} \\
& d C / d t=+c_{1} \cdot R_{1}-c_{2} \cdot R_{2}
\end{aligned}
$$

where

$$
\begin{array}{ll}
a_{1} & \text { is the SBML stoichiometry of } A \text { in reaction } 1 \\
a_{2} & \text { is the SBML stoichiometry of } A \text { in reaction } 2 \\
b_{1} & \text { is the SBML stoichiometry of } B \text { in reaction } 1 \\
c_{1} & \text { is the SBML stoichiometry of } C \text { in reaction } 1 \\
c_{2} & \text { is the SBML stoichiometry of } C \text { in reaction } 2
\end{array}
$$

We use the term SBML stoichiometries to highlight the fact that in this example involving mixed-units species, the values may not be identical to the biochemical stoichiometries in the reaction equations (9). And just what are the SBML stoichiometries? In the kind of mixed-units situation faced in this example, they must encompass both the biochemical stoichiometries and any necessary unit conversions. Thus, letting $m_{B}$ stand for the molecular mass of $B$ :

$$
\begin{array}{ll}
a_{1}=1000 & \text { (in each reaction event, } 1 \text { mole of } A \text { is consumed, with } A \text { expressed in millimoles) } \\
a_{2}=3000 & \text { (in each reaction event, } 3 \text { moles of } A \text { are produced, with } A \text { expressed in millimoles) } \\
b_{1}=2 \cdot m_{B} & \text { (in each reaction event, } 2 \text { moles of } B \text { are consumed, with } B \text { expressed in grams) } \\
c_{1}=6.02 \cdot 10^{23} & \text { (in each reaction event, } 1 \text { mole of } C \text { is produced, expressed as item counts) } \\
c_{2}=6.02 \cdot 10^{23} & \text { (in each reaction event, } 1 \text { mole of } C \text { is consumed, expressed as item counts) } \\
\text { and } k_{1}{ }^{\prime}=k_{1} \cdot 10^{-3} \cdot 1 / m_{B}{ }^{2} .
\end{array}
$$


What happens if the definition of the SBML built-in unit "substance" is changed in the model to be millimoles? Then the stoichiometries must be changed to the following:

$$
\begin{array}{rlrl}
a_{1} & =1 & & \text { (in each reaction event, } 1 \text { millimole of } A \text { is consumed, expressed in millimoles) } \\
a_{2} & =3 & & \text { (in each reaction event, } 3 \text { millimoles of } A \text { are produced, expressed in millimoles) } \\
b_{1} & =2 \cdot 10^{-3} \cdot m_{B} & & \text { (in each reaction event, } 2 \text { millimoles of } B \text { are consumed, expressed in grams) } \\
c_{1} & =6.02 \cdot 10^{20} & & \text { (in each reaction event, } 1 \text { millimole of } C \text { is produced, expressed as item counts) } \\
c_{2} & =6.02 \cdot 10^{20} & & \text { (in each reaction event, } 1 \text { millimole of } C \text { is consumed, expressed as item counts) } \\
\text { and } k_{1}{ }^{\prime}=k_{1} \cdot\left(10^{3} / m_{B}\right)^{2} . &
\end{array}
$$

What happens if instead the definition of the SBML built-in unit "substance" is changed in the model to be "item"? Then the stoichiometries must be changed to the following:

$$
\begin{array}{ll}
a_{1}=1 /\left(6.02 \cdot 10^{20}\right) & \text { (in each reaction event, } 1 \text { item of } A \text { is consumed, expressed in millimoles) } \\
a_{2}=3 /\left(6.02 \cdot 10^{20}\right) & \text { (in each reaction event, } 3 \text { items of } A \text { are produced, expressed in millimoles) } \\
b_{1}=2 \cdot 1 /\left(6.02 \cdot 10^{23}\right) \cdot m_{B} & \text { (in each reaction event, } 2 \text { items of } B \text { are consumed, expressed in grams) } \\
c_{1}=1 & \text { (in each reaction event, } 1 \text { item of } C \text { is produced, expressed as item counts) } \\
c_{2}=1 & \text { (in each reaction event, } 1 \text { item of } C \text { is consumed, expressed as item counts) }
\end{array}
$$

and $k_{1}{ }^{\prime}=k_{1} \cdot 6.02 \cdot 10^{20} \cdot\left(\frac{6.02 \cdot 10^{23}}{m_{B}}\right)^{2}$.

And finally, what happens if the definition of the SBML built-in unit "substance" is changed in the model to be "gram"? Then the stoichiometries must be changed again, to the following:

$$
\begin{array}{ll}
a_{1}=1000 \cdot m_{A} & \text { (in each reaction event, } 1 \text { gram of } A \text { is consumed, expressed in millimoles) } \\
a_{2}=3000 \cdot m_{A} & \text { (in each reaction event, } 3 \text { grams of } A \text { are produced, expressed in millimoles) } \\
b_{1}=2 & \text { (in each reaction event, } 2 \text { grams of } B \text { are consumed, expressed in grams) } \\
c_{1}=6.02 \cdot 10^{23} \cdot m_{C} & \text { (in each reaction event, } 1 \text { gram of } C \text { is produced, expressed as item counts) } \\
c_{2}=6.02 \cdot 10^{23} \cdot m_{C} & \text { (in each reaction event, } 1 \text { gram of } C \text { is consumed, expressed as item counts) }
\end{array}
$$

and $k_{1}{ }^{\prime}=k_{1} \cdot 10^{-3} \cdot m_{A}$, where $m_{A}, m_{B}$, and $m_{C}$ are the molecular masses of species $A, B$, and $C$, respectively.

In all of these cases, one straightforward approach to computing the value of $k_{1}{ }^{\prime}$ is to use an InitialAssignment construct for setting the value of $k_{1}{ }^{\prime}$ to the result of a formula such as one of those given in the examples above. And finally, note that if the species units were the same throughout (and in most models they are), the unit conversion aspects of the SBML stoichiometries would become unity, leaving only the expected biochemical stoichiometry values. Isn't that nice?

\subsubsection{Use of reaction identifiers in mathematical expressions}

The value of the id attribute of a Reaction can be used as the content of a ci element in MathML formulas elsewhere in the model. Such a ci element or symbol represents the rate of the given reaction as given by the reaction's KineticLaw object. The symbol has the units of substance/time.

A KineticLaw object in effect forms an assignment statement assigning the evaluated value of the math element to the symbol value contained in the Reaction id attribute. No other object can assign a value to such a reaction symbol; i.e., the variable attributes of InitialAssignment, RateRule, AssignmentRule and EventAssignment objects cannot contain the value of a Reaction id attribute.

The combined set of InitialAssignment, AssignmentRule and KineticLaw objects form a set of assignment statements that should be considered as a whole. The combined set of assignment rules should not contain algebraic loops: a chain of dependency between these statements should terminate. (More formally, consider the directed graph of assignment statements where nodes are statements and directed arcs exist for each occurrence of a symbol in a assignment statement math element. The directed arcs start from the statement defining the symbol to the statements that contain the symbol in their math elements. Such a graph must be acyclic.) Examples of valid and invalid set of assignment statements are given in Section 4.11.5. 


\subsection{Events}

Model has an optional list of Event objects that describe the time and form of explicit instantaneous discontinuous state changes in the model. For example, an event may describe that one species quantity is halved when another species quantity exceeds a given threshold value.

An Event object defines when the event can occur, the variables that are affected by the event, and how the variables are affected. The effect of the event can optionally be delayed after the occurrence of the condition which invokes it. The operation of an event is divided into two phases (even when the event is not delayed): one when the event is fired and the other when the event is executed. The Event type is defined in Figure 22. The object classes Event, Trigger, Delay and EventAssignment are derived from Sbase (see Section 3.2). An example of a model which uses events is given below.

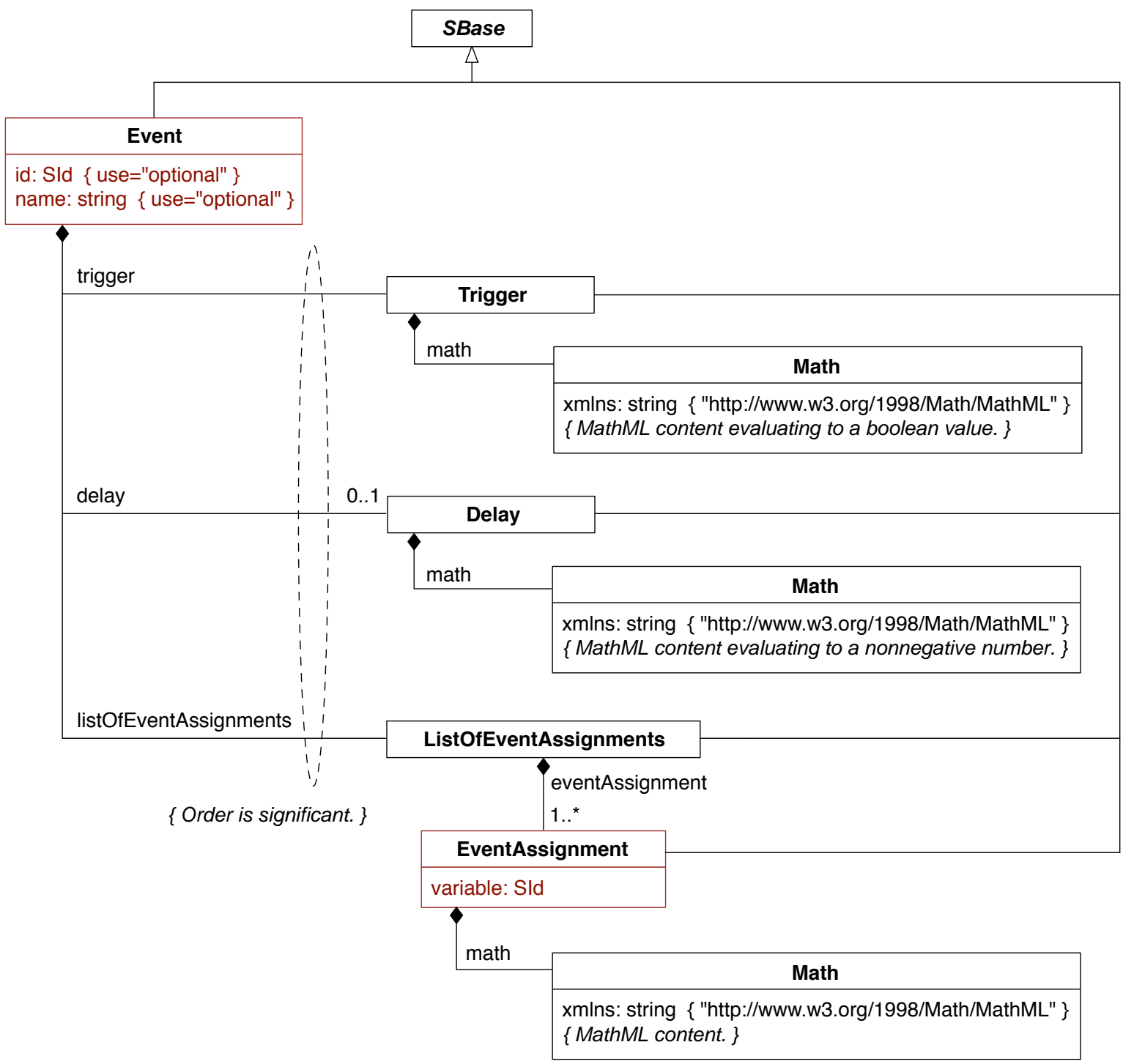

Figure 22: The definitions of Event, Trigger, Delay and EventAssignment, and the container class ListOfEventAssignment. 


\subsubsection{Event}

An Event definition has three required parts: an identifier, a trigger condition and at least one EventAssignment. In addition, an event can include an optional delay. These features of Event are described below.

Previous definitions of Event in SBML Level 2 included an additional attribute called timeUnits, which allowed the time units of the Delay to be set explicitly. SBML Level 2 Version 3 removes this attribute for several reasons. First, the ability to change the time units of the delay time of an Event to be different from the units of time for the whole model meant that computing an Event's time of triggering and its delay might have to be done using two different sets of units - a potential source of overlooked errors. Second, the ability to redefine the units of time for the delay of an Event became inconsistent with the lack of such an attribute on other SBML Level 2 Version 3 components involving an element of time, such as RateRule and KineticLaw. On balance, the timeUnits feature was judged to add needless complexity and inconsistency for little gain in functionality.

\section{The id and name attributes}

As with most components in SBML, an Event has id and name attributes, but in the case of Event, both are optional. These attributes operate in the manner described in Section 3.3.

\section{The optional sboTerm attribute on Event}

As with all SBML components derived from Sbase, an Event has an optional attribute sboTerm of type SBOTerm (see Sections 3.1.9 and 5). When a value is given to this attribute, it must be a valid term derived from SB0:0000231, "event" in SBO. The Event should have an "is a" relationship with the SBO term, and the term should be the most precise (narrow) term that captures the meaning of the event in the model.

As discussed in Section 5, SBO labels are optional information on a model. Applications are free to ignore sboTerm values. A model must be interpretable without the benefit of SBO labels.

\subsubsection{Trigger}

As shown in Figure 22, the trigger element of an Event must contain exactly one object of class Trigger. This object contains one math element containing a MathML expression. The expression must evaluate to a value of type boolean. The exact moment at which the expression evaluates to "true" is the time point when the Event is fired.

An event only fires when its Trigger expression makes the transition in value from "false" to "true". The event will also fire at any future time points when the trigger make this transition; in other words, an event can fire multiple times during a simulation if its trigger condition makes the transition from "false" to "true" more than once.

An important question is whether an event can fire prior to, or at, initial simulation time, i.e., $t \leq 0$. The answer is no: an event can only be triggered immediately after initial simulation time i.e., $t>0$.

\section{The optional sboTerm attribute on Trigger}

As with all SBML components derived from Sbase, Trigger inherits the optional attribute sboTerm of type SBOTerm (see Sections 3.1.9 and 5). When a value is given to this attribute, it must be a valid term representing a mathematical expression (i.e., a term chosen from the SB0:0000064, "mathematical expression" hierarchy). The formula in the Trigger's math expression should have an "is a" relationship with the SBO term, and the term should capture most precise (narrow) meaning of the mathematical formula of the trigger.

\subsubsection{Delay}

As shown in Figure 22, an Event object can contain an optional delay element of class Delay. The Delay is derived from Sbase and contains a mathematical formula stored in math. The formula is used to compute the length of time between when the event has fired and when the event's assignments (see below) are actually executed. If no delay is present on a given Event, a time delay of zero is assumed. 
The expression in math must be evaluated at the time the rule is fired. The expression must always evaluate to a nonnegative number (otherwise, a nonsensical situation could arise where an event is defined to fire before it is triggered!).

\section{Units of delay expressions}

The units of the numerical value computed by a Delay instance's math expression must match the model's units of time (meaning the definition of the "time" units in the model; see Section 4.4.3). Note that, as in other cases of MathML expressions in SBML, units are not predefined or assumed. As discussed in Section 3.4.10, literal numbers (i.e., numbers enclosed in MathML cn elements) or expressions containing only literal numbers and/or Parameter objects without declared units, are considered to have unspecified units. In such cases, the correspondence between the needed units and the (unknown) units of the Delay math expression cannot be proven, and while such expressions are not considered inconsistent, all that can be assumed by model interpreters (whether software or human) is that the units may be consistent.

The following Event example fragment helps illustrate this:

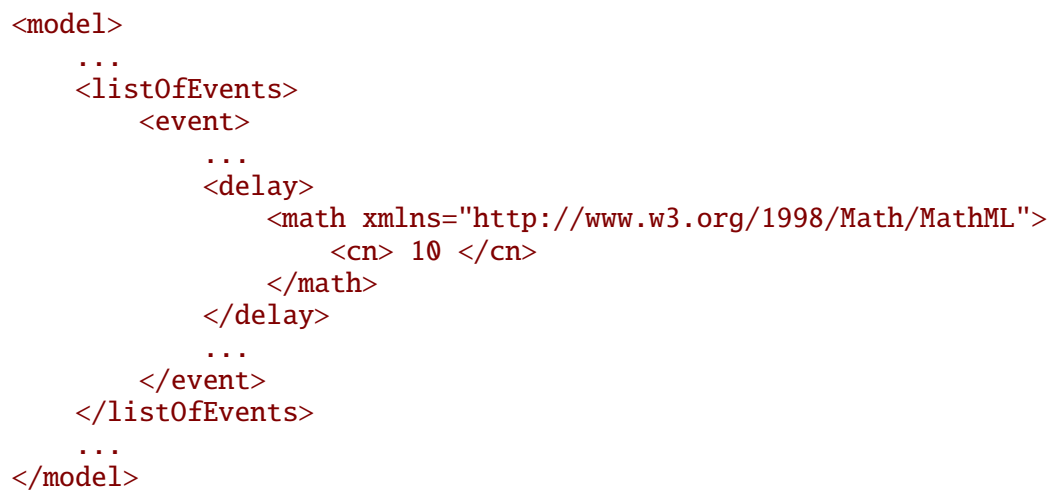

Note the " $<\mathrm{cn}>10</ \mathrm{cn}>$ " within the mathematical formula has no specified units. The model is not invalid because of this, but a recipient of the model may justifiably be concerned about what "10" really means. (Ten seconds? What if the global units of time on the model were changed from seconds to milliseconds? Would the modeler remember to change "10" to "10 000"?) As discussed elsewhere, leaving units unspecified may prevent software tools from performing complete validation and other useful operations such as global unit conversions. A better approach is to avoid literal numbers and instead use an approach such as defining a parameter with declared units, as in the following modified version of the example fragment:

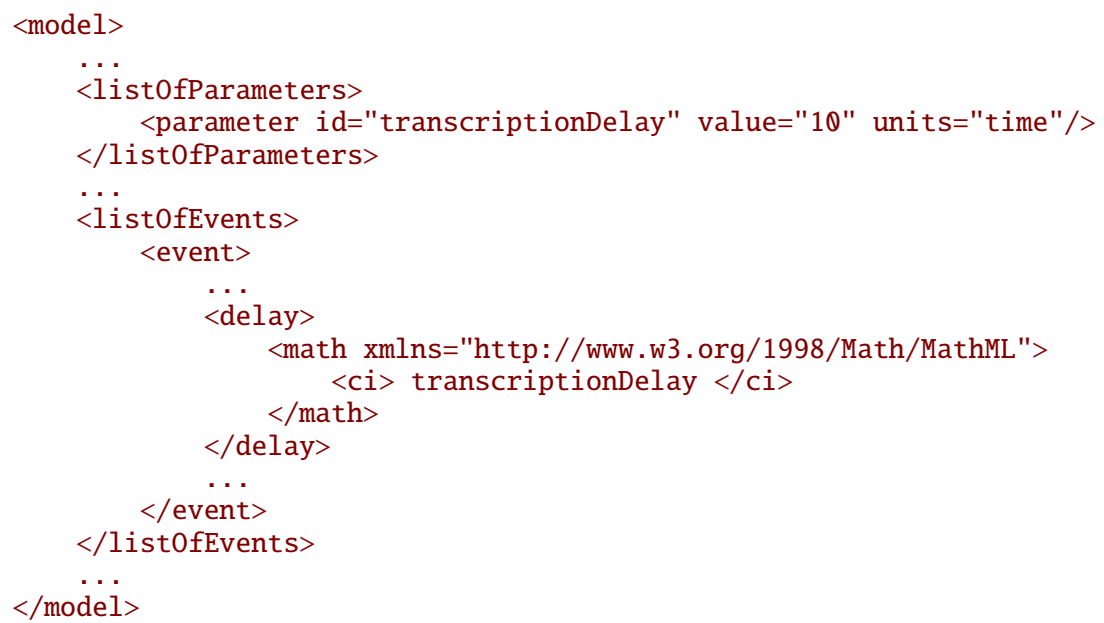




\section{The optional sboTerm attribute on Delay}

As with all SBML components derived from Sbase, Delay inherits an optional sboTerm attribute of type SBOTerm (see Sections 3.1.9 and 5). When a value is given to this attribute, it must be a valid term derived from the SB0:0000064, "mathematical expression" hierarchy in SBO. The Delay formula should have an "is a" relationship with the chosen SBO term, and the term should be the most precise (narrow) term that captures the meaning of the delay expression.

\subsubsection{EventAssignment}

Event contains a mandatory element called list0fEventAssignments, of class ListOfEventAssignment. In every instance of an event definition in a model, the object's list0fEventAssignments element must have a non-empty list of one or more eventAssignment elements of class EventAssignment. The object class EventAssignment has one required attribute, variable, and a required element, math. Being derived from Sbase, it also has all the usual attributes and elements of its parent class.

An "event assignment" has effect when the event is executed; that is, at the end of any given delay period (if given) following the moment that the Event is triggered. See Section 4.14.6 below for more information about events and event assignments in SBML.

\section{The variable attribute}

The variable attribute is of type SId and can contain the identifier of a Compartment, Species or Parameter instance defined in the model. When the event fires, the value of the model component identified by variable is changed by the EventAssignment to the value computed by the math element; that is, a species' quantity, compartment's size or parameter's value are reset to the value computed by math.

Certain restrictions are placed on what can appear in variable:

- The object identified by the value of the variable attribute must not have its constant attribute set to or default to "true". (Constants cannot be affected by events.)

- The variable attribute must not contain the identifier of a reaction; only species, compartment and parameter values may be set by an Event.

- The value of every variable attribute must be unique among the set of EventAssignment objects within a given Event instance. In other words, a single event cannot have multiple EventAssignments assigning the same variable. (All of them would be performed at the same time, when that particular Event triggers, resulting in indeterminacy.) Separate Event instances can refer to the same variable.

- A variable cannot be assigned a value in an EventAssignment object instance and also be assigned a value by an AssignmentRule, i.e., the value of the variable attribute in an EventAssignment instance cannot be the same as the value of a variable attribute in a AssignmentRule instance. (Assignment rules hold at all times, therefore it would be inconsistent to also define an event that reassigns the value of the same variable.)

\section{The optional sboTerm attribute on EventAssignment}

EventAssignment has an optional sboTerm attribute of type SBOTerm (see Sections 3.1.9 and 5). When a value is given to this attribute, it must be a valid SBO term identifier referring to a mathematical expression (i.e., terms derived from SB0:0000064, "mathematical expression"). The EventAssignment should have an "is a" relationship with the SBO term, and the term should be the most precise (narrow) term that captures the form of the assignment formula in the model.

\section{EventAssignment's math}

The math element contains a MathML expression that defines the new value of the variable. This expression is evaluated when the Event is fired but the variable only acquires the result or new value when the Event is executed. The order of the EventAssignment objects is not significant; the effect of one assignment cannot 
affect the result of another assignment. The identifiers occurring in the MathML ci attributes of the EventAssignment object represent the value of the identifier at the point when the Event is fired.

\section{Units of the math formula in EventAssignment}

In all cases, as would be expected, the units of the formula contained in the math element of EventAssignment must be consistent with the units of the object identified by the variable attribute. More precisely:

- In the case of a species, an EventAssignment sets the referenced species' quantity (concentration or amount of substance) to the value determined by the formula in math. The units of the math formula must be identical to the units of the species as defined in Section 4.8.5.

- In the case of a compartment, an EventAssignment sets the referenced compartment's size to the size determined by the formula in math. The overall units of the formula must be identical to the units specified for the size of the compartment identified by the value of the EventAssignment's variable attribute. (See Section 4.7.5 for an explanation of how the units of the compartment's size are determined.)

- In the case of a parameter, an EventAssignment sets the referenced parameter's value to that determined by the formula in math. The overall units of the formula must be identical to the units defined for the parameter identified by the value of the EventAssignment's variable attribute. (See Section 4.9.3 for an explanation of how the units of the parameter are determined.)

Note that the formula placed in the math element has no assumed units. The consistency of the units of the formula, and the units of the entity which the assignment affects, must be explicitly established just as in the case of the value of delay. An approach similar to the one discussed in Section 4.14.3 may be used.

\subsubsection{Example Event definitions}

A example of an Event object follows. This structure makes the assignment $k_{2}=0$ at the point when $P_{1} \leq P_{2}$ :

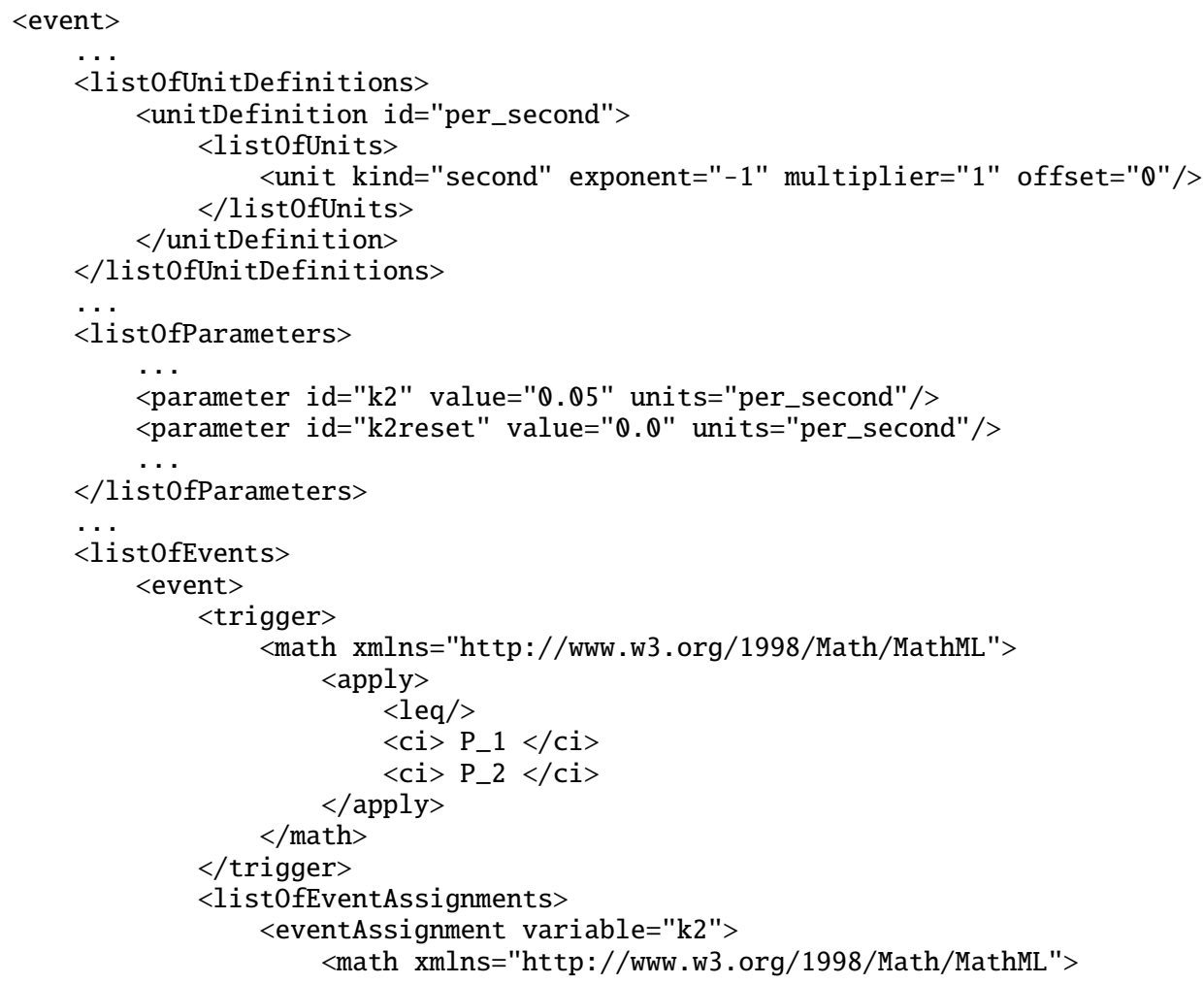




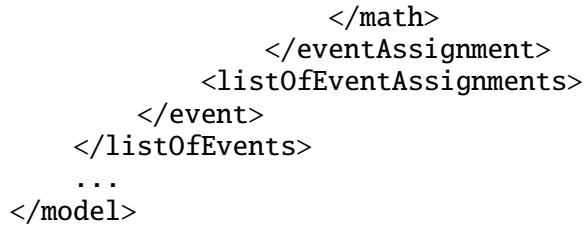

A complete example of a model using events is given in Section 7.10.

\subsubsection{Detailed semantics of events}

The description of events above describes the action of events in isolation from each other. This section describes how events interact.

Events whose trigger expression is true at the start of a simulation do not fire at the start of the simulation $(t=0)$. Events fire only when the trigger becomes true, i.e., the trigger expression transitions from false to true, which cannot happen at $t=0$ but can happen at $t>0$.

Any transition of a trigger expression from "false" to "true" will cause an event to fire. Consider an event $E$ with delay $d$ where the trigger expression makes a transition from false to true at times $t_{1}$ and $t_{2}$. The EventAssignment object will have effect at $t_{1}+d$ and $t_{2}+d$ irrespective of the relative times of $t_{1}$ and $t_{2}$. For example events can "overlap" so that $t_{1}<t_{2}<t_{1}+d$ still causes an event assignments to occur at $t_{1}+d$ and $t_{2}+d$.

It is possible for events to fire other events, i.e., an event assignment can cause an event to fire, therefore it is possible for a model to be entirely encoded in Event objects.

It is entirely possible for two events to be executed simultaneously in simulated time. It is assumed that, although the precise time at which these events are executed is not resolved beyond the given point in simulated time, the order in which the events occur is resolved. This order can be significant in determining the overall outcome of a given simulation. SBML Level 2 does not define the algorithm for determining this order (the tie-breaking algorithm). As a result, the outcomes of simulations involving events may vary when simultaneous events occur during simulation. It is anticipated that future versions or levels of SBML will define a specific set of tie-breaking algorithms and a mechanism for models to indicate which algorithm should be applied during simulation.

Despite the absence of a specific tie-breaking algorithm, SBML event simulation is constrained as follows. When an event $X$ fires another event $Y$ and event $Y$ has zero delay then event $Y$ is added to the existing set of simultaneous events that are pending execution. Events such as $Y$ do not have a special priority or ordering within the tie-breaking algorithm. Events $X$ and $Y$ form a cascade of events at the same point in simulation time. All events in a model are open to being in a cascade. The position of an event in the event list does not affect whether it can be in the cascade: $Y$ can be triggered whether it is before or after $X$ in the list of events. A cascade of events can be infinite (never terminate). When this occurs a simulator should indicate this has occurred; i.e., it is incorrect for the simulator to arbitrarily break the cascade and continue the simulation without at least indicating the infinite cascade occurred. A variable can change more than once when processing simultaneous events at simulation time $t$. The model behavior (output) for such a variable is the value of the variable at the end of processing all the simultaneous events at time $t$. 


\section{The Systems Biology Ontology and the sboTerm attribute}

The values of id attributes on SBML components allow the components to be cross-referenced within a model. The values of name attributes on SBML components provide the opportunity to assign them meaningful labels suitable for display to humans (Section 3.3). The specific identifiers and labels used in a model necessarily must be unrestricted by SBML, so that software and users are free to pick whatever they need. However, this freedom makes it more difficult for software tools to determine, without additional human intervention, the semantics of models more precisely than the semantics provided by the SBML object classes defined in other sections of this document. For example, there is nothing inherent in a parameter with identifier "k" that would indicate to a software tool it is a first-order rate constant (if that's what "k" happened to be in some given model). However, one may need to convert a model between different representations (e.g., Henri-Michaelis-Menten vs. elementary steps), or to use it with different modelling approaches (discrete or continuous). One may also need to relate the model components with other description formats, using a deeper semantics. Although an advanced software tool might be able to deduce the semantics of some model components through detailed analysis of the kinetic rate expressions and other parts of the model, this quickly becomes infeasible for any but the simplest of models.

An approach to solving this problem is to associate model components with terms from carefully curated controlled vocabularies $(\mathrm{CVs})$. This is the purpose of the optional sboTerm attribute provided on the SBML class Sbase. The sboTerm attribute always refers to terms belonging to the Systems Biology Ontology (SBO, http://biomodels.net/SBO/). In this section, we discuss the sboTerm attribute, SBO, the motivations and theory behind their introduction, and guidelines for their use.

SBO is not part of SBML; it is being developed separately, to allow the modeling community to evolve the ontology independently of SBML. However, the terms in the ontology are being designed with SBML components in mind, and are classified into subsets that can be directly related with SBML components such as reaction rate expressions, parameters, and a few others. The use of sboTerm attributes is optional, and the presence of sboTerm on an element does not change the way the model is interpreted. Annotating SBML elements with SBO terms adds additional semantic information that may be used to convert the model into another model, or another format. Athough SBO support provides an important source of information to understand the meaning of a model, software does not need to support sboTerm to be considered SBMLcompliant.

\subsection{Principles}

Labeling model components with terms from shared controlled vocabularies allows a software tool to identify each component using identifiers that are not tool-specific. An example of where this is useful is the desire by many software developers to provide users with meaningful names for reaction rate equations. Software tools with editing interfaces frequently provide these names in menus or lists of choices for users. However, without a standardized set of names or identifiers shared between developers, a given software package cannot reliably interpret the names or identifiers of reactions used in models written by other tools.

The first solution that might come to mind is to stipulate that certain common reactions always have the same name (e.g., "Michaelis-Menten"), but this is simply impossible to do: not only do humans often disagree on the names themselves, but it would not allow for correction of errors or updates to the list of predefined names except by issuing new releases of the SBML specification - to say nothing of many other limitations with this approach. Moreover, the parameters and variables that appear in rate expressions also need to be identified in a way that software tools can interpret mechanically, implying that the names of these entities would also need to be regulated.

The Systems Biology Ontology (SBO) provides vocabularies for identifying common reaction rate expressions, common participant types and roles in reactions, common parameter types and their roles in rate expressions, common modeling frameworks (e.g., "continuous", "discrete", etc.), and common types of species and reactions. The relationship implied by an sboTerm value on an SBML model component is "is a": the thing defined by that SBML component "is an" instance of the thing defined in SBO by the term having the identifier given by the sboTerm attribute value. By adding SBO term references on the components of a 
model, a software tool can provide additional details using independent, shared vocabularies that can enable other software tools to recognize precisely what the component is meant to be. Those tools can then act on that information. For example, if the SBO identifier SB0:0000049 is assigned to the concept of "first-order irreversible mass-action kinetics, continuous framework", and a given KineticLaw object in a model has an sboTerm attribute with this value, then regardless of the identifier and name given to the reaction itself, a software tool could use this to inform users that the reaction is a first-order irreversible mass-action reaction. This kind of reverse engineering of the meaning of reactions in a model would be difficult to do otherwise, especially for more complex reaction types.

The presence of an SBO label on a compartment, species, or reaction, can help map SBML elements to equivalents in other standards, such as (but not limited to) BioPAX (http://www.biopax.org/) or the Systems Biology Graphical Notation (SBGN, http://www.sbgn.org/). Such mappings can be used in conversion procedures, or to build interfaces, with SBO becoming a kind of "glue" between standards of representation.

The presence of the label on a kinetic expression can also allow software tools to make more intelligent decisions about reaction rate expressions. For example, an application could recognize certain types of reaction formulas as being ones it knows how to solve with optimized procedures. The application could then use internal, optimized code implementing the rate formula indexed by identifiers such as SB0:0000049 appearing in SBML models.

Although the use of SBO can be beneficial, it is critical to keep in mind that the presence of an sboTerm value on an object must not change the fundamental mathematical meaning of the model. An SBML model must be defined such that it stands on its own and does not depend on additional information added by SBO terms for a correct mathematical interpretation. SBO term definitions will not imply any alternative mathematical semantics for any SBML object labeled with that term. Two important reasons motivate this principle. First, it would be too limiting to require all software tools to be able to understand the SBO vocabularies in addition to understanding SBML Level 2 Version 3. Supporting SBO is not only additional work for the software developer; for some kinds of applications, it may not make sense. If SBO terms on a model are optional, it follows that the SBML model must remain unambiguous and fully interpretable without them, because an application reading the model may ignore the terms. Second, we believe allowing the use of sboTerm to alter the mathematical meaning of a model would allow too much leeway to shoehorn inconsistent concepts into SBML objects, ultimately reducing the interoperability of the models.

\subsection{Using SBO and sboTerm}

The sboTerm attribute data type is always SBOTerm, defined in Section 3.1.9. When present in a given model object instance, the attribute's value must be an identifier taken from the Systems Biology Ontology (SBO; http://biomodels.net/SBO/). This identifier must refer to a single SBO term that best defines the entity encoded by the SBML object in question. An example of the type of relationship intended is: the KineticLaw in reaction $R 1$ is a first-order irreversible mass action rate law.

Note the careful use of the words "defines" and "entity encoded by the SBML object" in the paragraph above. As mentioned, the relationship between the SBML object and the URI is:

The "thing" encoded by this SBML object is an instance of the "thing" represented by the referenced SBO term.

\subsubsection{The structure of the Systems Biology Ontology}

The goal of SBO labeling for SBML is to clarify to the fullest extent possible the nature of each element in a model. The approach taken in SBO begins with a hierarchically-structured set of controlled vocabularies with five main divisions: (1) modeling framework, (2) participant type, (3) quantitative parameter, (4) mathematical expression, and (5) event. Figure 23 on the following page illustrates the highest level of SBO.

Each of the five branches of Figure 23 have a hierarchy of terms underneath them. At this time, we can only begin to list some initial concepts and terms in SBO; what follows is not meant to be complete, comprehensive or even necessarily consistent with future versions of SBO. The web site for SBO 
(http://biomodels.net/SBO/) should be consulted for the current version of the ontology. Section 5.4.1 describes how the impact of SBO changes on software applications is minimized.

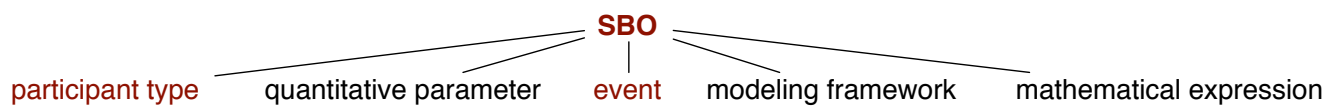

Figure 23: The five controlled vocabularies (CVs) that make up the main branches of SBO. (Other CVs in SBO may evolve in time, but are not discussed here.)

Figure 24 shows the structure for the participant type CV which reflects the hierarchical conceptual groupings of the concepts. For example, in reaction rate expressions, there are a variety of possible modifiers. Some classes of modifiers can be further subdivided and grouped. All of this is easy to capture in the CV. As more agreement is reached in the modeling community about how to define and name modifiers for different cases, the $\mathrm{CV}$ can grow to accommodate it.

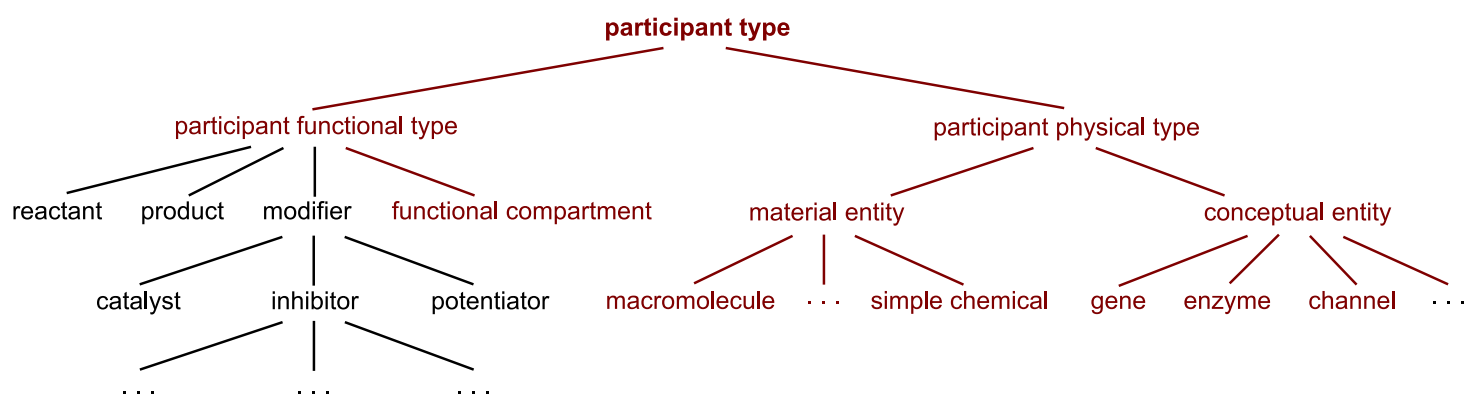

Figure 24: Partial expansion of some of the terms in the participant type CV of SBO.

Similar to the above, the controlled vocabulary for quantitative parameters also has a hierarchical structure, as illustrated in Figure 25. Note the separation of kinetic constant into separate terms for unimolecular, bimolecular, etc. reactions, as well as for forward and reverse reactions. The need to have separate terms for forward and reverse rate constants arises in reversible mass-action reactions. This distinction is not always necessary for all quantitative parameters; for example, there is no comparable concept for the Michaelis constant. Another distinction for some quantitative parameters is a decomposition into different versions based on the modeling framework being assumed. For example, different terms for continuous and discrete formulations of kinetic constants represent specializations of the constants for particular simulation frameworks. Not all quantitative parameters will need to be distinguished along this dimension.

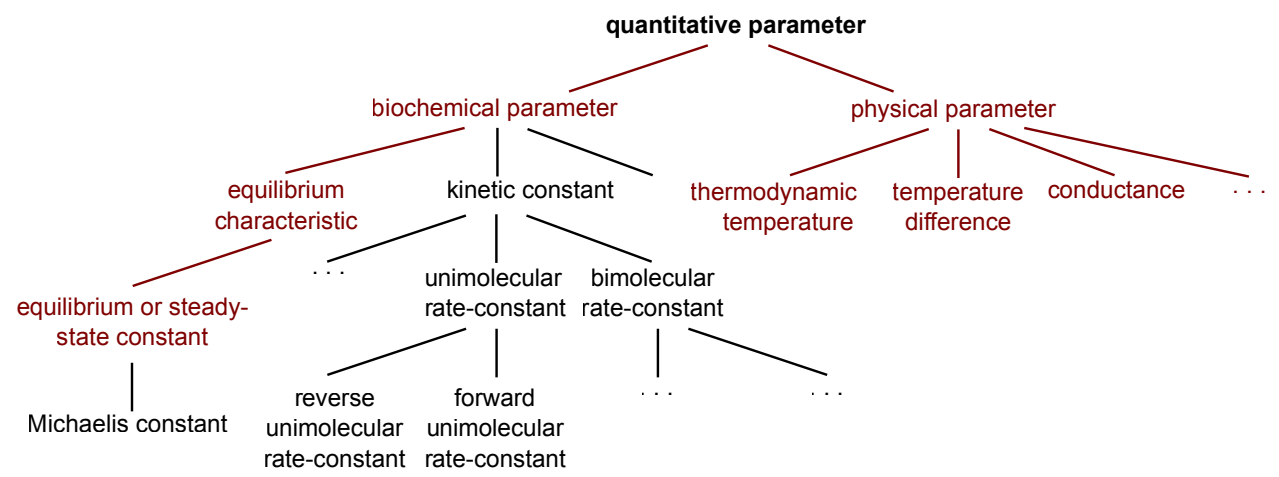

Figure 25: Partial expansion of some of the terms in the quantitative parameter $\mathrm{CV}$. 
The terms of the SBO quantitative parameter vocabulary contain mathematical formulas encoded using MathML 2.0 expressing the parameter using other SBO parameters. The main use of that approach is to avoid listing all the variants of a mathematical expression, escaping a combinatorial explosion.

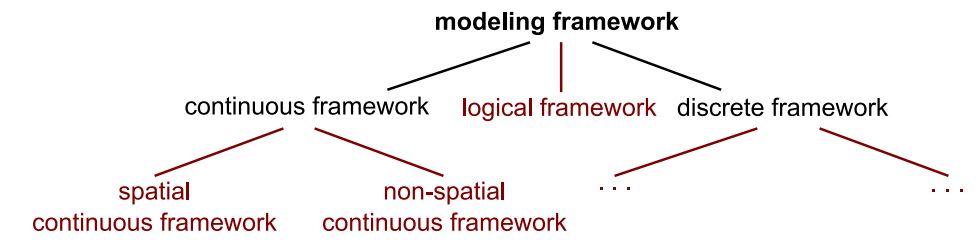

Figure 26: Partial expansion of some of the terms in the modeling framework $\mathrm{CV}$.

The modeling framework controlled vocabulary is needed to support labeling models and reactions with the framework for which they are designed. Figure 26 illustrates the structure of this CV, which is at this point extremely simple, but we expect that more terms will evolve in the future.

The mathematical expression vocabulary encompasses the various mathematical expressions that constitute a model. Figure 27 illustrates a portion of the hierarchy. Rate law formulas are part of the mathematical expression hierarchy, and subdivided by successively more refined distinctions until the leaf terms represent precise statements of common reaction types. Other types of mathematical expressions are likely to be included in the future in order to be able to characterize other mathematical components of a model, namely initial assignments, assignment rules, rate rules, algebraic rules, constraints, and event triggers and assignments.

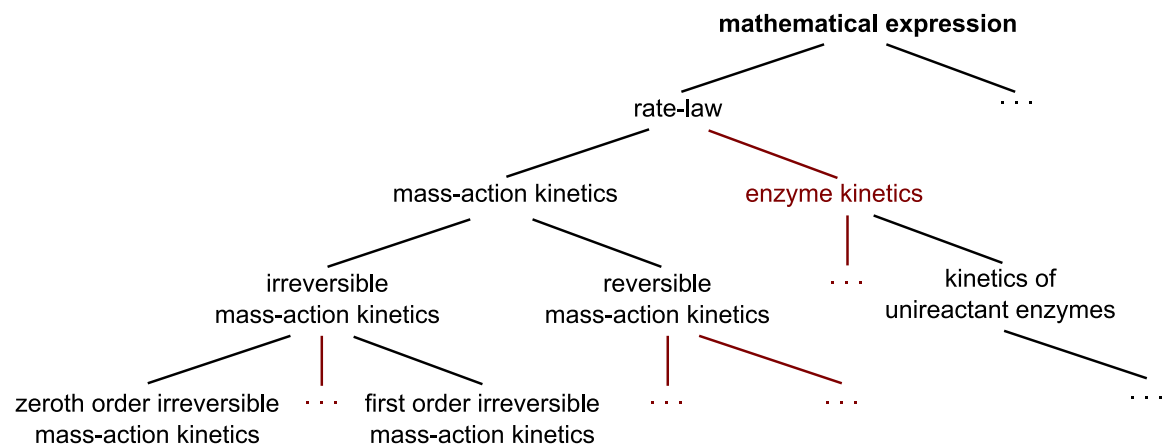

Figure 27: Partial expansion of some of the terms in the mathematical expression CV.

The leaf terms of the rate law portion of the SBO mathematical expression framework contain the mathematical formulas for the rate laws encoded using MathML 2.0. There are many potential uses for this. One is to allow a software application to obtain the formula and insert it into a model. In effect, the formulas given in the CV act as templates for what to put into an SBML KineticLaw definition. The MathML definition also acts as a precise statement about the rate law in question. Some of the non-leaf terms also contain rate laws encoded using MathML 2.0. However, those are generic rate-laws, containing MathML constructs not yet supported by SBML and need to be expanded into the MathML subset allowed in SBML before they can be used in conjunction with SBML models.

To make this discussion concrete, here is an example definition of an entry in the SBO rate law hierarchy at the time of this writing. This term represents second-order, irreversible, mass-action kinetics with one reactant, formulated for use in a continuous modeling framework:

\section{ID: SBO:0000052}

Name: second-order irreversible mass action kinetics, one reactant, continuous scheme. 
Definition: Reaction scheme where the products are created from the reactants and the change of a product quantity is proportional to the product of reactant activities. The reaction scheme does not include any reverse process that creates the reactants from the products. The change of a product quantity is proportional to the square of one reactant quantity. It is to be used in a reaction modelled using a continuous framework.

Parent(s):

- SB0:0000050 second order irreversible mass action kinetics, one reactant (is a).

- SB0:0000163 irreversible mass action kinetics, continuous scheme (is a).

MathML:

<math xmlns="http://www.w3.org/1998/Math/MathML">

<semantics definitionURL="http://biomodels.net/SBO/\#SBO:0000062">

$<$ lambda $>$

$<$ bvar $><$ ci definitionURL="http://biomodels.net/SBO/\#SBO:0000036" $>\mathrm{k}</$ ci $></$ bvar $>$

$<$ bvar $><$ ci definitionURL="http://biomodels.net/SBO/\#SBO:0000010" $>\mathrm{R}</$ ci $></$ bvar $>$

$<$ apply>

$<$ times $/>$

$<\mathrm{ci}>\mathrm{k}</ \mathrm{ci}>$

$<\mathrm{Ci}>\mathrm{R}</ \mathrm{ci}>$

$<\mathrm{Ci}>\mathrm{R}</ \mathrm{ci}>$

$</$ apply $>$

$</$ lambda $>$

$</$ semantics $>$

$</$ math $>$

In the MathML definition of the term shown above, the bound variables in the lambda expression are tagged with references to terms in the SBO quantitative parameter (for $\mathbf{k}$ ) and SBO participant role (for R) vocabularies. This makes it possible for software applications to interpret the intended meanings of the parameters in the expression.

The event branch of SBO defines types of biological processes. It lists the types of biochemical reactions, such as binding, conformational transition, or cleavage, and also the different controls that modify a biochemical reaction, such as inhibition, catalysis, etc.

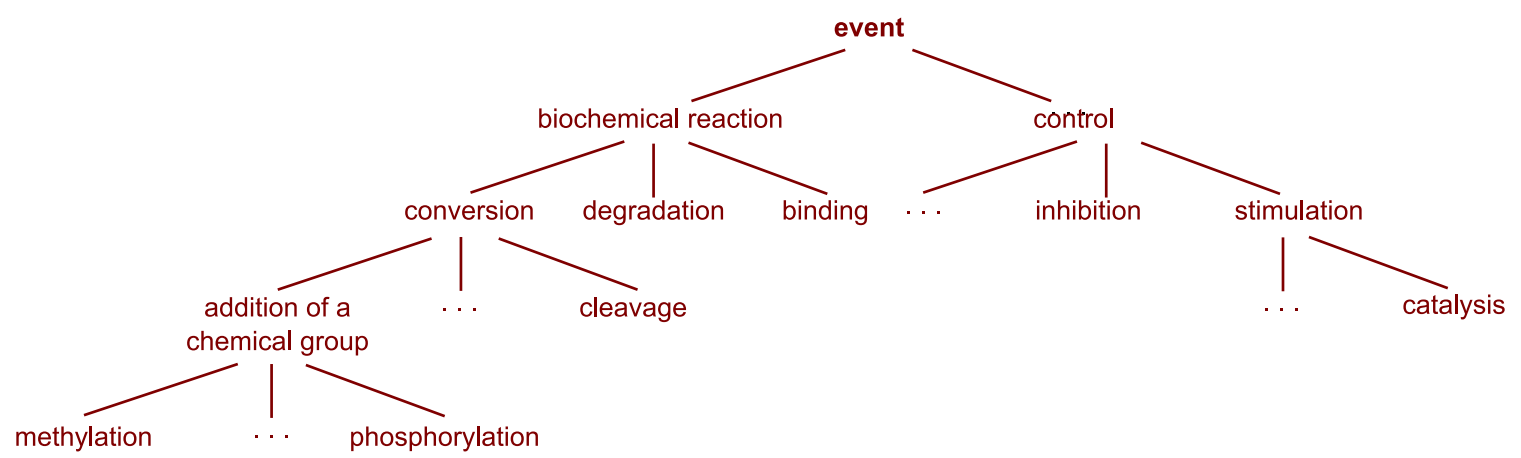

Figure 28: Partial expansion of some of the terms in the event $C V$.

One of the goals of SBO is to permit a tool to traverse up and down the hierarchy in order to find equivalent terms in different frameworks. The hope is that when a software tool encounters a given rate formula in a model, the formula will be a specific form (say, "mass-action kinetics, second order, one reactant, for discrete simulation"), but by virtue of the consistent organization of the reaction rate CV into framework-specific definitions, the tool should in principle be able to determine the definitions for other frameworks (say, "massaction kinetics, second order, one reactant for continuous simulation"). If the software tool is designed for continuous simulation and it encounters an SBML model with rate laws formulated for discrete simulation, it could in principle look up the rate laws' identifiers in the CV and search for alternative definitions intended for discrete simulation. And of course, the converse is true, for when a tool designed for discrete simulation encounters a model with rate laws formulated for continuous simulation. 


\subsubsection{Relationships between individual SBML components and SBO terms}

The sboTerm attribute is defined on the abstract class Sbase and can be used in all derived elements. However, not all SBO terms can be used to annotate all SBML elements. Table 6 summarizes the relationship between SBML components and the branches within SBO that apply to that component. (There are currently no specific SBO term that correspond to the Sbml, UnitDefinition, Unit, and various ListOf list classes).

\begin{tabular}{lll}
\hline SBML Component & SBO Vocabulary & Parent SBO Identifier \\
\hline Model & modeling framework & SB0:0000004 \\
FunctionDefinition & mathematical expression & SB0:0000064 \\
CompartmentType & participant physical type & SB0:0000236 \\
SpeciesType & participant physical type & SB0:0000236 \\
Compartment & participant physical type & SB0:0000236 \\
Species & participant physical type & SB0:0000236 \\
Reaction & event & SB0:0000231 \\
Parameter & quantitative parameter & SB0:0000002 \\
SpeciesReference & participant functional type & SB0:0000003 \\
ModifierSpeciesReference & participant functional type & SB0:0000003 \\
KineticLaw & rate law & SB0:0000001 \\
InitialAssignment & mathematical expression & SB0:0000064 \\
AlgebraicRule & mathematical expression & SB0:0000064 \\
AssignmentRule & mathematical expression & SB0:0000064 \\
RateRule & mathematical expression & SB0:0000064 \\
Constraint & mathematical expression & SB0:0000064 \\
Event & event & SB0:0000231 \\
Trigger & mathematical expression & SB0:0000064 \\
Delay & mathematical expression & SB0:0000064 \\
EventAssignment & mathematical expression & SB0:0000064 \\
\hline
\end{tabular}

Table 6: SBML components and the main types of SBO terms that may be assigned to them. The parent identifiers are provided for guidance, but actual annotations should use more specific child terms. See text for explanation.

The parent identifiers shown in Table 6 are provided for reference. They are the highest-level terms in their respective vocabularies; however, these are not the terms that would be used to annotate an element in SBML, because there are more specific terms underneath the parents shown here. A software tool should use the most specific SBO term available for a given concept rather than using the top-level identifier acting as the root of that particular vocabulary.

\subsubsection{Tradeoffs in using SBO terms}

The SBO-based approach to annotating SBML components with controlled terms has the following strengths:

1. The syntax is minimally intrusive and maximally simple, requiring only one string-valued attribute.

2. It supports a significant fraction of what SBML users have wanted to do with controlled vocabularies.

3. It does not interfere with any other scheme. The more general annotation-based approach described in Section 6 can still be used simultaneously in the same model.

The scheme has the following weaknesses:

1. An object can only have one sboTerm attribute, therefore, it can only be related to a single term in SBO. (This also impacts the design of SBO: it must be structured such that a class of SBML elements can logically only be associated with one class of terms in the ontology.)

2. The only relationship that can be expressed by sboTerm is "is a". It is not possible to represent different relationships (known as verbs in ontology-speak). This limits what can be expressed using SBO. 
The weaknesses are not shared by the annotation scheme described in Section 6. If application's needs cannot be met using SBO terms, software developers should examine the approach described in Section 6.

\subsection{Relationships to the SBML annotation element}

Another means of providing this kind of information would be to place SBO terms inside the annotation element defined on Sbase (Sections 3.2 and 6). However, in the interest of making the use of SBO in SBML maximally interoperable between software tools, the best-practice recommendation is to place SBO references in the sboTerm attribute rather than inside the annotation element of an object.

Although sboTerm is just another kind of optional annotation in SBML, the SBO references are separated out into their own attribute on SBML components, both to simplify their use for software tools and because they assert a slightly stronger and more focused connection in a more regimented fashion. SBO term references are intended to allow a modeler to make a statement of the form "this object is identical in meaning and intention to the object defined in the term X of SBO", and do so in a way that a software tool can interpret unambiguously.

Some software applications may have their own vocabulary of terms similar in purpose to SBO. For maximal software interoperability, the best-practice recommendation in SBML is nonetheless to use SBO terms in preference to using application-specific annotation schemes. Software applications should therefore attempt to translate their private terms to and from SBO terms when writing and reading SBML, respectively.

\subsection{Discussion}

Here we discuss some additional points about the SBO-based approach.

\subsubsection{Frequency of change in the ontology}

The SBO development approach follows conventional ontology development approaches in bioinformatics. One of the principles being followed is that identifiers and meanings of terms in the CVs never change and the terms are never deleted. Where some terms are deemed obsolete, the introduction of new terms refine or supersede existing terms, but the existing identifiers are left in the CV. Thus, references never end up pointing to nonexistent entries. In the case where synonymous terms are merged after agreement that multiple terms are identical, the term identifiers are again left in the CV and they still refer to the same concept as before. Out-of-date terms cached or hard-coded by an application remain usable in all cases. (Moreover, machine-readable CV encodings and appropriate software design should render possible the development of API libraries that automatically map older terms to newer terms as the CVs evolve.)

Therefore, a model is never in danger of ending up with SBO identifiers that cannot be dereferenced. If an application finds an old model with a term SB0:0000065, it can be assured that it will be able to find this term in SBO, even if it has been superseded by other, more preferred terms.

\subsubsection{Consistency of information}

If you have a means of linking (say) a reaction rate formula to a term in a $\mathrm{CV}$, it is possible to have an inconsistency between the formula in the SBML model and the one defined for the CV term. However, this is not a new problem; it arises in other situations involving SBML models already. The guideline for these situations is that the model must be self-contained and stand on its own. Therefore, in cases where they differ, the definitions in the SBML model take precedence over the definitions referenced by the CV. In other words, the model (and its MathML) is authoritative.

\subsubsection{Implications for network access}

A software tool does not need to have the ability to access the network or read the CV every time it encounters a model or otherwise works with SBML. Since the SBO will likely stabilize and change infrequently once a core set of terms is defined, applications can cache the controlled vocabulary, and not make network accesses to the master SBO copy unless something forces them to (e.g., detecting a reference in a model 
to an SBO term that the application does not recognize). Applications could have user preference settings indicating how often the $\mathrm{CV}$ definitions should be refreshed (similar to how modern applications provide a setting dictating how often they should check for new versions of themselves). Simple applications may go further and hard code references to terms in SBO that have reached stability and community consensus. SBO is available for download under different formats (http://biomodels.net/SBO/). Web services are also available to provide programmatic access to the ontology.

\subsubsection{Implications for software tools}

If a software tool does not pay attention to the SBO annotations described here, one is faced with exactly the situation that exists today: the SBML model must be interpreted as-is, without benefit of the information added by the SBO terms. The purpose of introducing an ontology scheme and guidelines for its use is to give tools enough information that they could perform added processing, if they were designed to take advantage of that information. 


\section{A standard format for the annotation element}

This section describes the recommended non-proprietary format for the content of annotation elements in SBML when (a) referring to controlled vocabulary terms and database identifiers which define and describe biological and biochemical entities, and (b) describing the creator of a model and its modification history. Such a structured format should facilitate the generation of models compliant with the MIRIAM guidelines for model curation (Le Novère et al., 2005).

This format should not be used to refer to SBO terms (Section 5), because SBO defines terms about mathematical modeling constructs and not the biological and biochemical entities that the mathematics represent.

The format described in this section is intended to be the form of one of the top-level elements that could reside in an annotation element attached to an SBML object derived from Sbase. The element is named rdf:RDF. The format described here is compliant with the constraints placed on the form of annotation elements described in Section 3.2.4. We refer readers to Section 3.2.4 for important information on the structure and organization of application-specific annotations; these are not described here.

\subsection{Motivation}

The SBML structures described elsewhere in this document do not have any biochemical or biological semantics. The format described in this section provides a scheme for linking SBML structures to external resources so that those structures can have such semantics. The motivation for the introduction of this scheme is similar to that given for the introduction of sboTerm; however, this scheme is significantly more flexible.

\subsection{XML namespaces in the standard annotation}

This format uses a restricted form of Dublin Core (Dublin Core Metadata Initiative, 2005) and BioModels qualifier elements (see http://www.biomodels.net/index.php?s=Qualifiers) embedded in RDF (W3C, 2004b). It uses a number of external XML standards and associated XML namespaces. Table 7 lists these namespaces and relevant documentation on those namespaces. The format constrains the order of elements in these namespaces beyond the constraints defined in the standard definitions for those namespaces. For each standard listed, the format only uses a subset of the possible syntax defined by the given standard. Thus it is possible for an annotation element to include XML that is compliant with those external standards but is not compliant with the format described here. Parsers wishing to support this format should be aware that a valid annotation element may contain an rdf:RDF element which is not compliant with the format described here. A parser should check that all aspects of the syntax defined here before assuming that the contained data is encoded in the format.

\begin{tabular}{lll}
\hline & Namespace URI & Definition Document \\
\hline dc & http://purl.org/dc/elements/1.1/ & Powell and Johnston (2003) \\
rdf & http://www.w3.org/1999/02/22-rdf-syntax-ns\# & W3C (2004a) \\
dcterms & http://purl.org/dc/terms/ & Kokkelink and Schwänzl (2002) \\
& & DCMI Usage Board (2005) \\
vcard & http://www.w3.org/2001/vcard-rdf/3.0\# & Iannella (2001) \\
bqbiol & http://biomodels.net/biology-qualifiers/ & \\
bqmodel & http://biomodels.net/model-qualifiers/ & \\
\hline
\end{tabular}

Table 7: The XML standards used in the SBML standard format for annotation. The namespace prefix are shown to indicate only the prefix used in the main text. 


\subsection{General syntax for the standard annotation}

An outline of the format syntax is shown below.

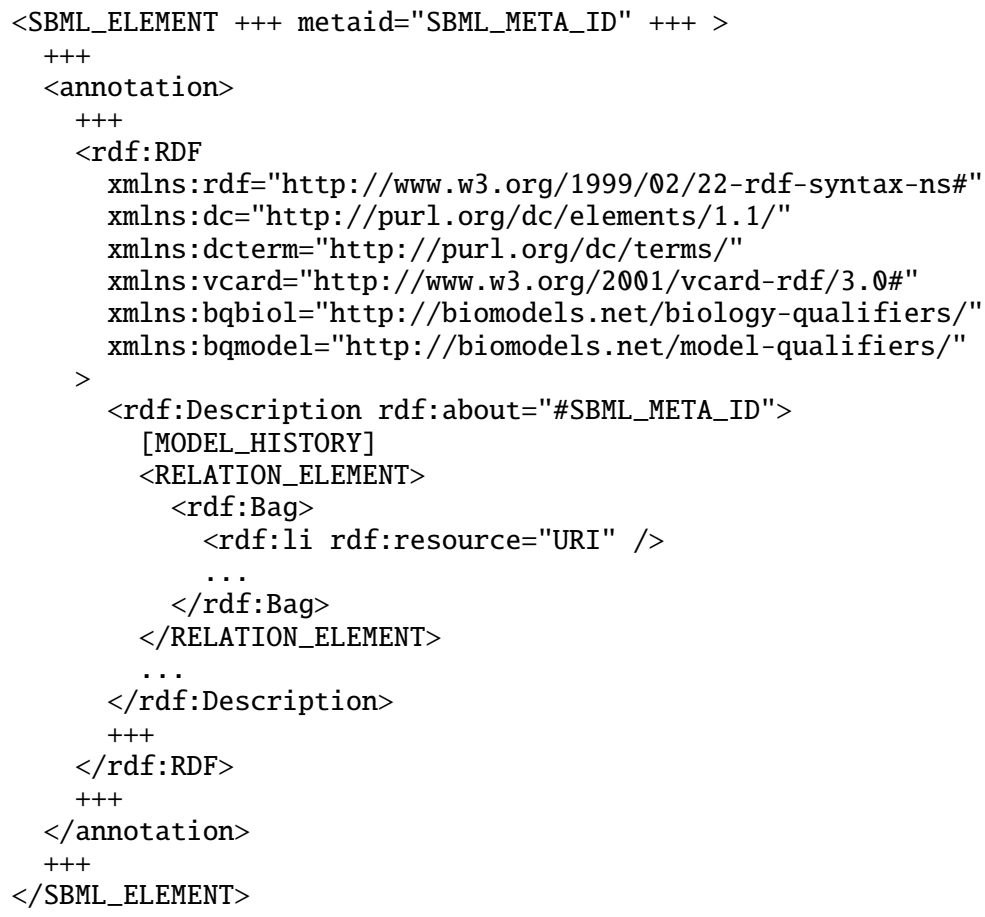

The above outline shows the order of the elements. The capitalized identifiers refer to generic strings of a particular type: SBML_ELEMENT refers to any SBML element name that can contain an annotation element; SBML_META_ID is a XML ID string; RELATION_ELEMENT refers to element names in either the namespace http://biomodels.net/biology-qualifiers/ or http://biomodels.net/model-qualifiers/; and URI is a URI. [MODEL_HISTORY] refers to an optional section described in Section 6.6 which can only be present within SBML model elements. ' +++ ' is a placeholder for either no content or valid XML syntax that is not defined by the standard annotation scheme but is consistent with the relevant standards for the enclosing elements. '. . ' is a placeholder for zero or more elements of the same form as the immediately preceding element. The precise form of whitespace and the XML namespace prefix definitions is not constrained; however, the elements and attributes must be in the namespaces shown. The rest of this section describes the format formally in English.

In this format, the annotation of an element is located in a single rdf:RDF element contained within an SBML annotation element. The annotation element can contain other elements in any order as described in Section 3.2.4. The format described in this section only defines the form of the rdf:RDF element. The containing SBML Sbase element must have a metaid attribute value. (As this attribute is of the type ID its value must unique to the entire SBML document.)

The first element of the rdf:RDF element must be an rdf: Description element with an rdf: about attribute. (This format doesn't define the form of subsequent elements of the rdf:RDF element.) The value of the rdf: about attribute must be of the form \#<string > where the string component is equal to the value of the metaid attribute of the containing SBML element.

The rdf:Description element can contain only an optional model history section (see Section 6.6) followed by a sequence of zero or more BioModels relation elements. The optional model history section can only be present within an SBML Model element. The specific type of the relation elements will vary depending on the relationship between the SBML component and referenced information or resource.

Although Section 6.5 describes the detailed semantics of each of the relation element types, the content of these elements follows the same form. The BioModels qualifiers relation elements must only contain a single 
rdf: Bag element which in turn must only contain one or more rdf: li elements. The rdf:li elements must only have a rdf: resource attribute containing a URI referring to an information resource (See Section 6.4).

Annotations in this format can be located at different depths within a model component.

\subsection{Use of URIs}

The format represents a set of relationships between the SBML element and the resources referred to by the contained rdf:resource attribute values. The BioModels relation elements simply define the type of the relationship.

For example, a Species element representing a protein could be annotated with a reference to the database UniProt by the http://www. uniprot.org/\#P12999 resource identifier, identifying exactly the protein described by the Species element. This identifier maps to a unique entry in UniProt which is never deleted from the database. In the case of UniProt, this is the "accession" of the entry. When the entry is merged with another one, both "accession" are conserved. Similarly in a controlled vocabulary resource, each term is associated with a perennial identifier. The UniProt entry also possess an "entry name" (the Swiss-Prot "identifier"), a "protein name", "synonyms" etc. Only the "accession" is perennial and should be used.

The value of a rdf:resource attribute is a URI that both uniquely identifies the resource, and the data in the resource. In this case the resource constraining the identifier precedes the '\#' symbol and the term or database identifier follows the '\#' symbol. In the above example, the resource http://www.uniprot.org/ includes the entry P12999.

The value of the rdf:resource attribute is a URI, not a URL; as such, a URI does not have to reference a physical web object but simply identifies a controlled vocabulary term or database object (a URI is a label that, in this case, just happens to look like a URL). For instance, a true URL for an Internet resource such as http://www . uniprot.org/entry/P12999 might correspond to the URI http://www. uniprot.org/\#P12999.

SBML does not specify how a parser is to interpret a URI. In the case of a transformation into a physical URL, there could be several solutions. For example, the URI http://www. geneontology .org/\#G0:0007268 can be translated into:

http://www . ebi .ac.uk/ego/DisplayGoTerm?selected=G0:0007268

http://www . godatabase.org/cgi-bin/amigo/go.cgi?view=details\&query=G0: 0007268

http://www. informatics. jax.org/searches/GO.cgi?id=G0:0007268

Similarly the URI http://www.ec-code.org/\#3.5.4.4 can refer to:

http://www. ebi .ac .uk/intenz/query?cmd=SearchEC\&ec=3.5.4.4

http://www. expasy.org/cgi-bin/nicezyme.pl?3.5.4.4

http://www. chem. qmul.ac.uk/iubmb/enzyme/EC3/5/4/4.html

http://www.genome.jp/dbget-bin/www_bget?ec:3.5.4.4

etc.

To enable interoperability, the community has agreed on an initial set of standardized valid URI syntax rules which may be used within the standard annotation format. This set of rules is not part of the SBML standard but will grow independently from specific SBML Levels and Versions. As the set changes a given URI syntax rule will not be modified although the physical resource associated with the rule may change. These URIs will always be composed as resource\#id. The MIRIAM resources http://www.ebi.ac.uk/compneur-srv/ miriam-main/ lists URI syntaxes and possible physical links to controlled vocabulary and databases. Each entry contains a list of SBML and relation elements in which the given URI can be appropriately embedded. To enable consistent and thus useful links to external resources, the URI syntax rule set must have a consistent view of the concepts represented by the different SBML elements for the purposes of this format. For example as the rule set is designed to link SBML biological and biochemical resources the rule set assumes that a Species element represents the concept of a biochemical entity type rather than mathematical symbol. The URI rule list will evolve with the evolution of databases and resources. The annotation format described in this section does not require a simple parser of this format to access this list. 


\subsection{Relation elements}

To enable the format to encode different types of relationships between SBML elements and resources, different BioModel qualifier elements are used to enclose a set of rdf:li elements. The relation elements imply a specific relationship between the enclosing SBML element and the resources referenced by the rdf:li elements.

The detailed semantics (i.e. from the perspective of automatic parser) of the relation elements is defined by the URI list at http://www.ebi.ac.uk/compneur-srv/miriam-main/, and thus is outside the scope of SBML. The URI list generally assumes that the biological entity represented by the element is the concept linked to the reference resource.

Several relation elements with a given tag, enclosed in the same SBML element, each represent an alternative annotation to the SBML element. For example two bqbiol:hasPart elements within a Species SBML element represent two different sets of references to the parts making up the the chemical entity represented by the species. (The species is not made up of all the entities represented by all the references combined).

The complete list of the qualifier elements in the Biomodels qualifier namespaces is documented at http://www.biomodels.net/index.php?s=Qualifiers. The list is divided into two namespaces one for model qualifiers http://biomodels.net/biology-qualifiers/ (prefix used here bqbiol) and the other for biological qualifiers http://biomodels.net/model-qualifiers/) (prefix used here bqmodel. This list will only grow i.e no element will be removed from the list. The following is the list of elements at the time of writing:

- bqmodel:is The modeling object encoded by the SBML component is the subject of the referenced resource. For instance, this qualifier might be used to link the model to a model database.

- bqmodel:isDescribedBy The modeling object encoded by the SBML component is described by the referenced resource. This relation might be used to link SBML components to the literature that describes this model or this kinetic law.

- bqbiol: is The biological entity represented by the SBML component is the subject of the referenced resource. This relation might be used to link a reaction to its exact counterpart in (e.g.) KEGG or Reactome.

- bqbiol: hasPart The biological entity represented by the SBML component includes the subject of the referenced resource, either physically or logically. This relation might be used to link a complex to the description of its components.

- bqbiol:isPart0f The biological entity represented by the SBML component is a physical or logical part of the subject of the referenced resource. This relation might be used to link a component to the description of the complex is belongs to.

- bqbiol:isVersion0f The biological entity represented by the SBML component is a version or an instance of the subject of the referenced resource.

- bqbiol:hasVersion The subject of the referenced resource is a version or an instance of the biological entity represented by the SBML component.

- bqbiol:isHomologTo The biological entity represented by the SBML component is homolog, to the subject of the referenced resource, i.e. they share a common ancestor.

- bqbiol:isDescribedBy The biological entity represented by the SBML component is described by the referenced resource. This relation should be used, for example, to link a species or a parameter to the literature that describes the quantity of the species or the value of the parameter.

\subsection{Model history}

When enclosed in an SBML Model element, the format described in previous sections can include additional elements to describe the history of the model. This history data must occur immediately before the first 
BioModels relation elements. These additional elements encode information on the model creator and a sequence of dates recording changes to the model. The syntax for this section is outlined below.

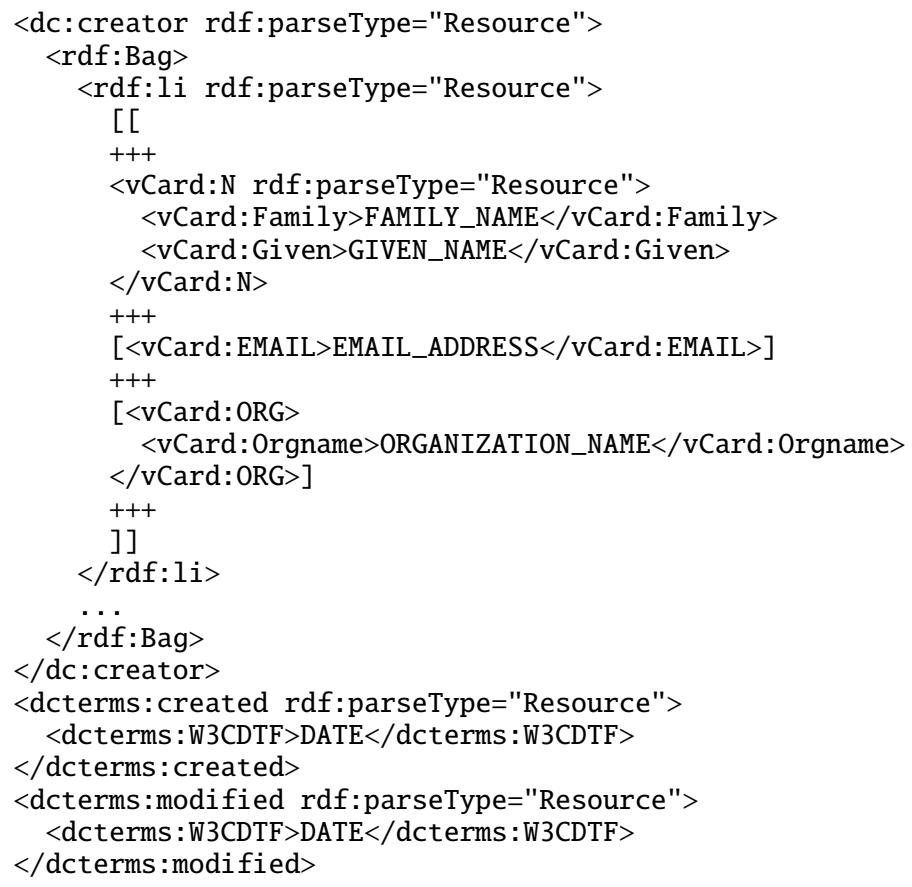

The order of elements is as shown above except that elements of the format contained between [ [ and ]] can occur in any order. The capitalized identifiers refer to generic strings of a particular type: FAMILY_NAME is the family name of a person who created the model; GIVEN_NAME is the first name of the same person who created the model; EMAIL_ADDRESS is the email address of the same person who created the model; and ORGANIZATION_NAME is the name of the organization with which the same person who created the model is affiliated DATE is a date in W3C date format (Wolf and Wicksteed, 1998). W3CDTF, N, ORG and EMAIL are literal strings. The elements of the format contained between [ and ] are optional. '+++' is a placeholder for either no content or valid XML syntax that is not defined by the standard annotation scheme but is consistent with the relevant standards for the enclosing elements. '.. ' is a placeholder for zero or more elements of the same form as the immediately preceding element. The precise form of whitespace and the XML namespace prefix definitions is not constrained. The remaining text in this section describes the syntax formally in English.

The additional elements of the model history sub-format consist in sequence of a dc:creator element, a dcterms:created element and zero or more dcterms:modified elements. All these elements must have the attribute rdf:parseType set to Resource.

The dc:creator element describes the person who created the SBML encoding of the model and contains a single rdf:Bag element. The rdf:Bag element can contain any number of elements; however, the first element must be a rdf:li element. The rdf:li element can contain any number of elements in any order. The set of elements contained with the rdf:li element can include the following informative elements: vCard:N, vCard:EMAIL and vCard:ORG. The vCard:N contains the name of the creator and must consist of a sequence of two elements: vCard:Family and the vCard:Given whose content is the family (surname) and given (first) names of the creator respectively. The vCard:N must have the attribute rdf: parseType set to Resource. The content of the vCard:EMAIL element must be the email address of the creator. The content of the vCard:ORG element must contain a single vCard:Orgname element. The vCard:Orgname element must contain the name of an organization to which the creator is affiliated.

The dcterms:created and dcterms : modified elements must each contain a single dcterms:W3CDTF element whose content is a date in W3C date format (Wolf and Wicksteed, 1998) which is a a profile of (restricted form of) ISO 8601. 


\subsection{Examples}

The following shows the annotation of a model with model creation data and links to external resources:

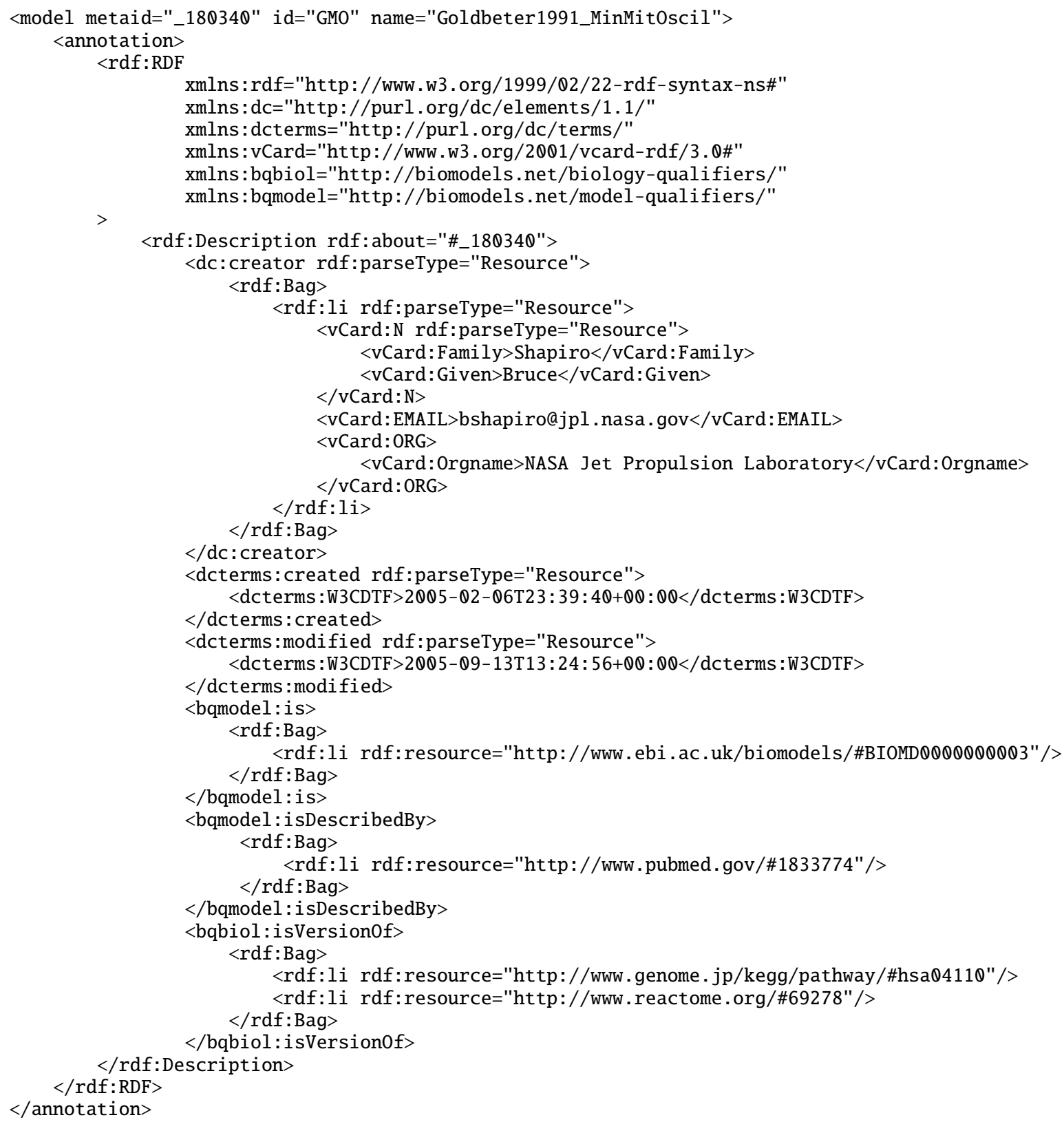

The following example shows a Reaction structure annotated with a reference to its exact Reactome counterpart.

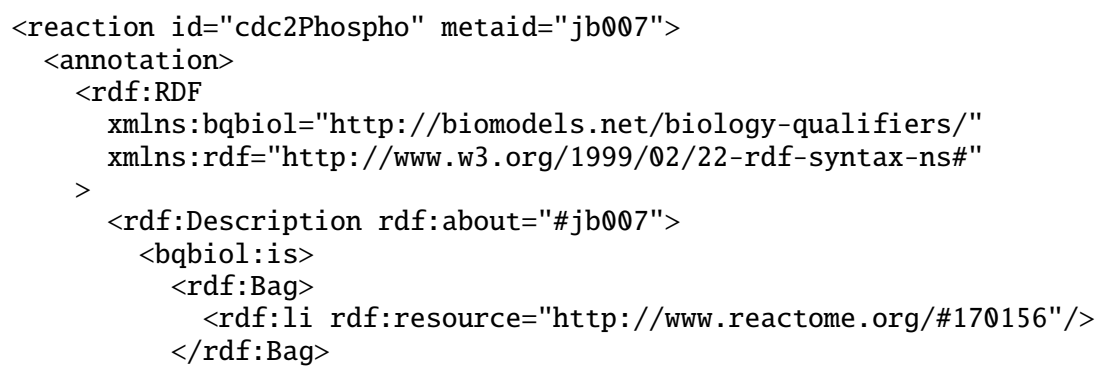




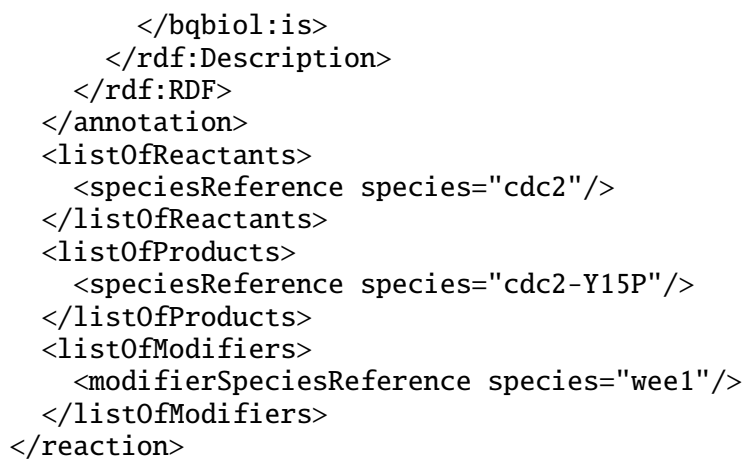

The following example describes a species that represents a complex between the protein calmodulin and calcium ions:

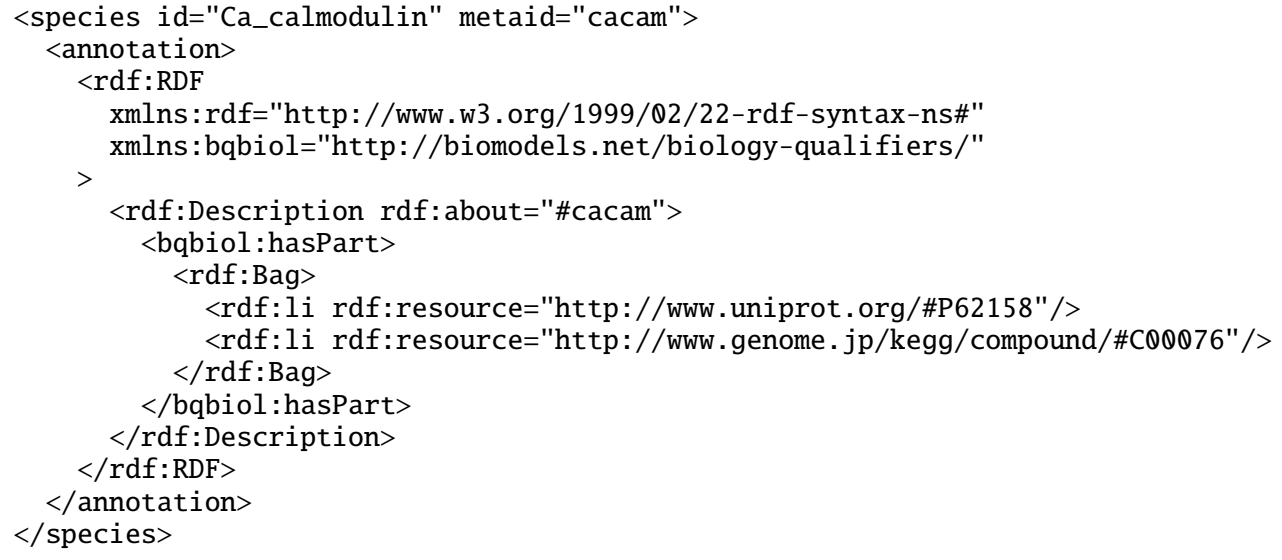

The following example describes a species that represents either "Calcium/calmodulin-dependent protein kinase type II alpha chain" or "Calcium/calmodulin-dependent protein kinase type II beta chain". This is the case, for example, in the somatic cytoplasm of striatal medium-size spiny neurons, where both are present but they cannot be functionally differentiated.

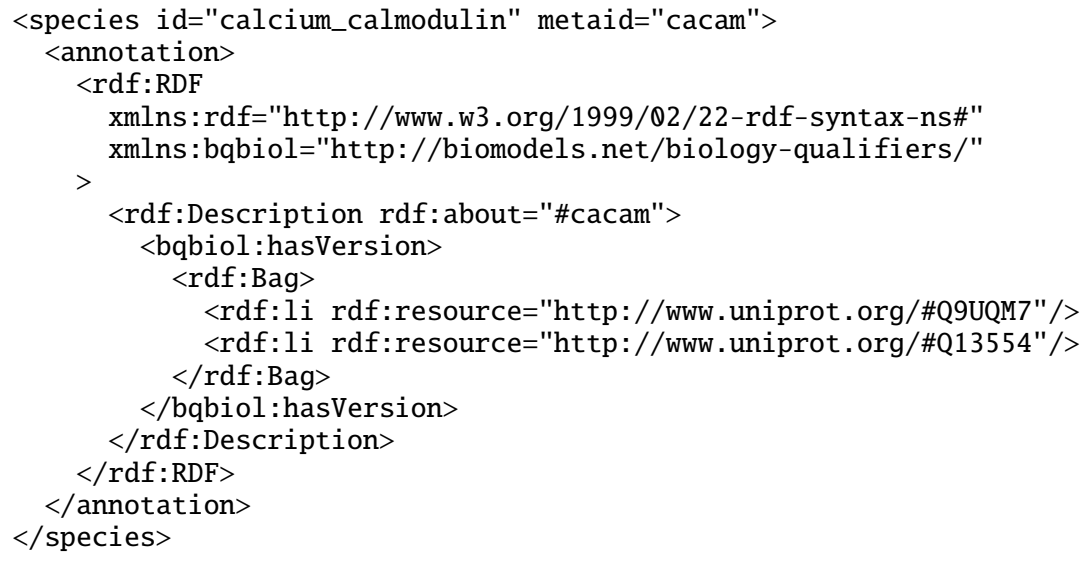

The above approach should not be used to describe "any Calcium/calmodulin-dependent protein kinase type II chain", because such an annotation requires referencing the products of other genes such as gamma or delta. All the known proteins could be enumerated, but such an approach would almost surely lead to inaccuracies because biological knowledge continues to evolve. Instead, the annotation should refer to generic information such as Ensembl family ENSF00000000194 "CALCIUM/CALMODULIN DEPENDENT KINASE TYPE II CHAIN" or PIR superfamily PIRSF000594 "Calcium/calmodulin-dependent protein kinase type II". 
The following two examples show how to use the qualifier isVersionof. The first example is the relationship between a reaction and an EC code. An EC code describes an enzymatic activity and an enzymatic reaction involving a particular enzyme can be seen as an instance of this activity. For example, the following reaction represents the phosphorylation of a glutamate receptor by a complex calcium/calmodulin kinase II.

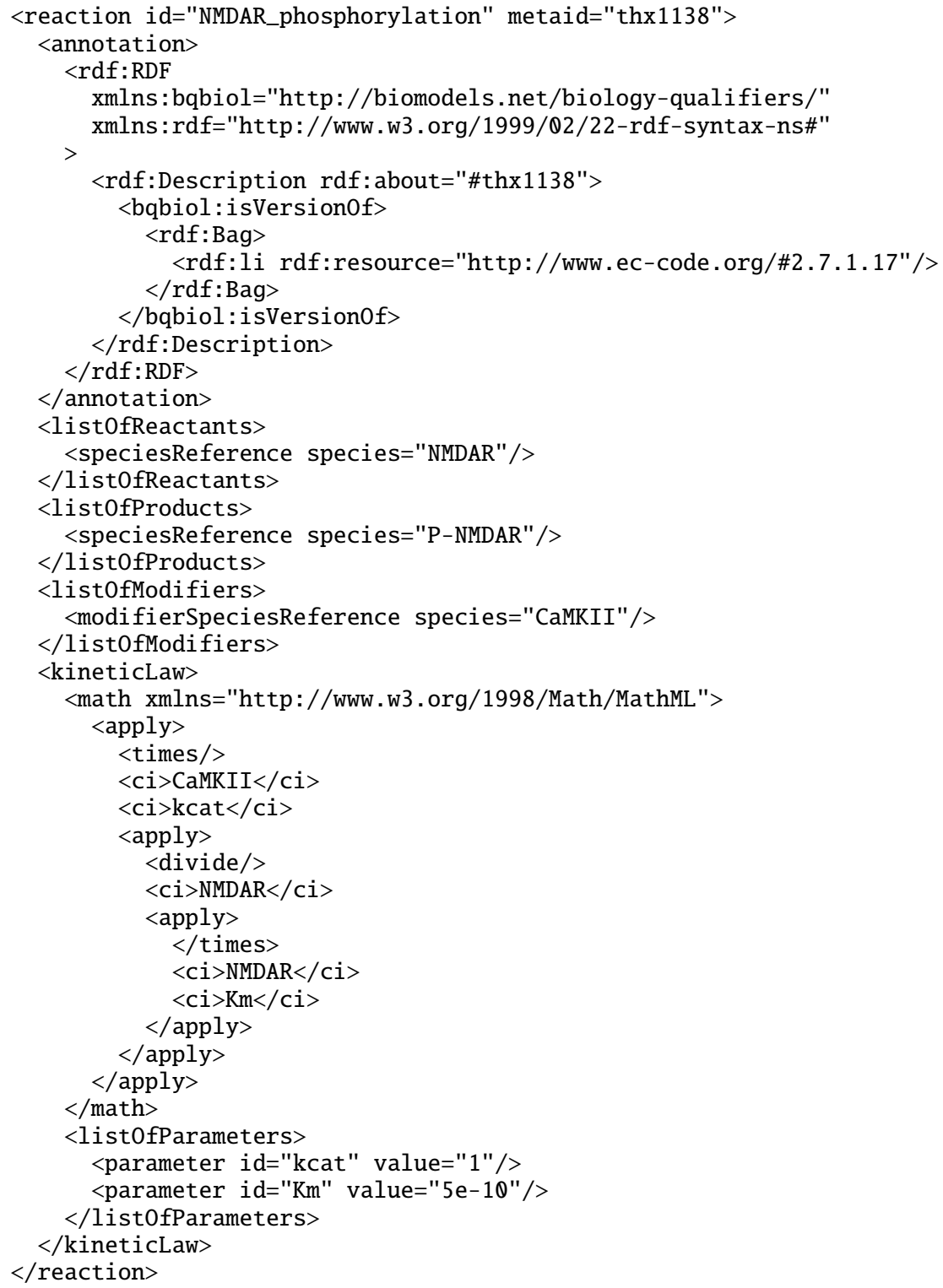

The second example of the use of isVersionof is the complex between Calcium/calmodulin-dependent protein kinase type II alpha chain and Calcium/calmodulin, that is only one of the "calcium- and calmodulindependent protein kinase complexes" described by the Gene Ontology term GO:0005954.

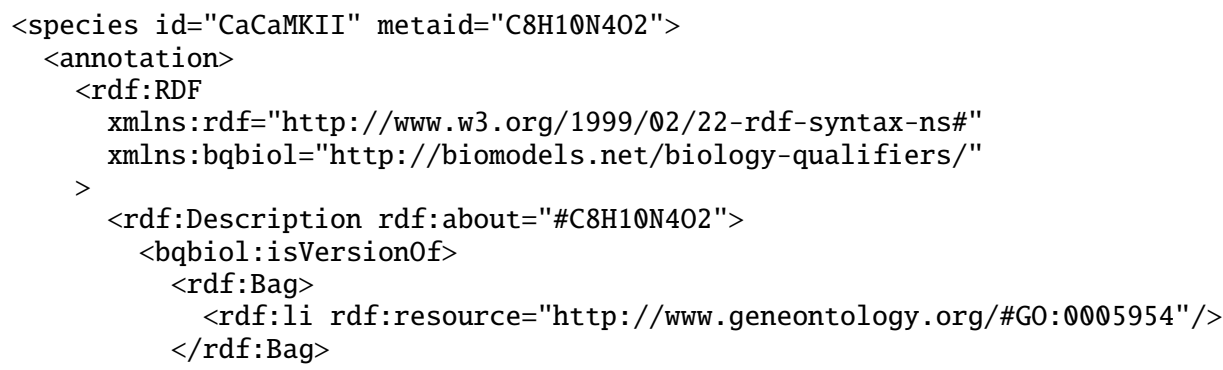




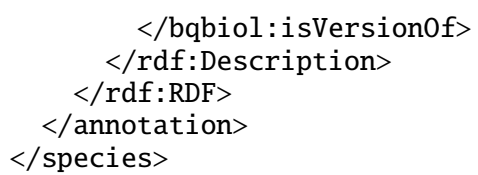

The previous case is different form the following one, although they could seem similar at first sight. The "Calcium/calmodulin-dependent protein kinase type II alpha chain" is a part of the above mentioned "calcium- and calmodulin-dependent protein kinase complex".

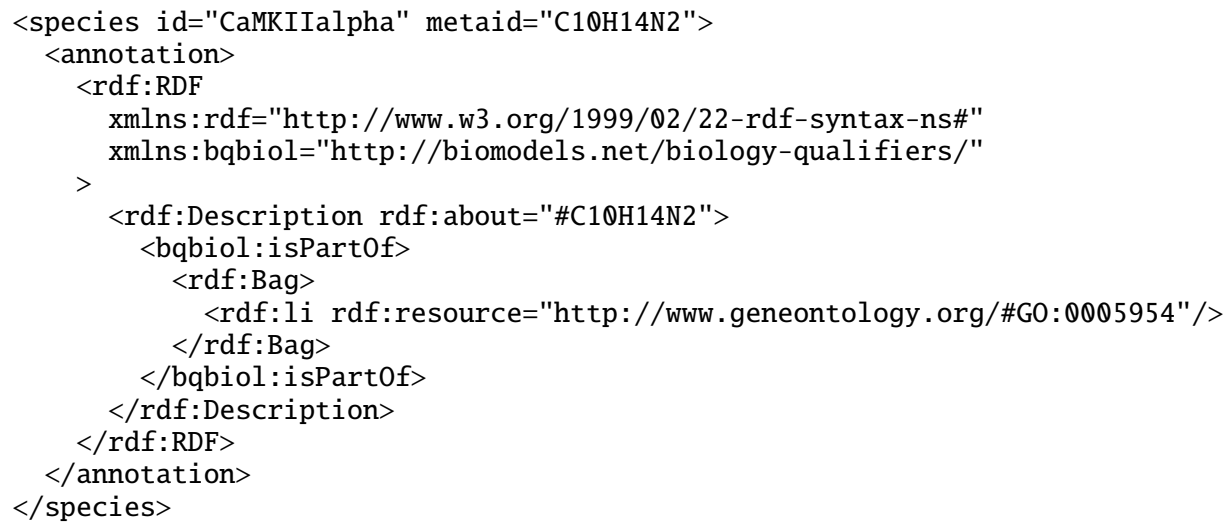

It is possible describe a component with several alternative sets of qualified annotations. For example, the following species represents a pool of GMP, GDP and GTP. We annotate it with the three corresponding KEGG compound identifiers but also with the three corresponding ChEBI identifiers. The two alternative annotations are encoded in separate hasVersion qualifier elements.

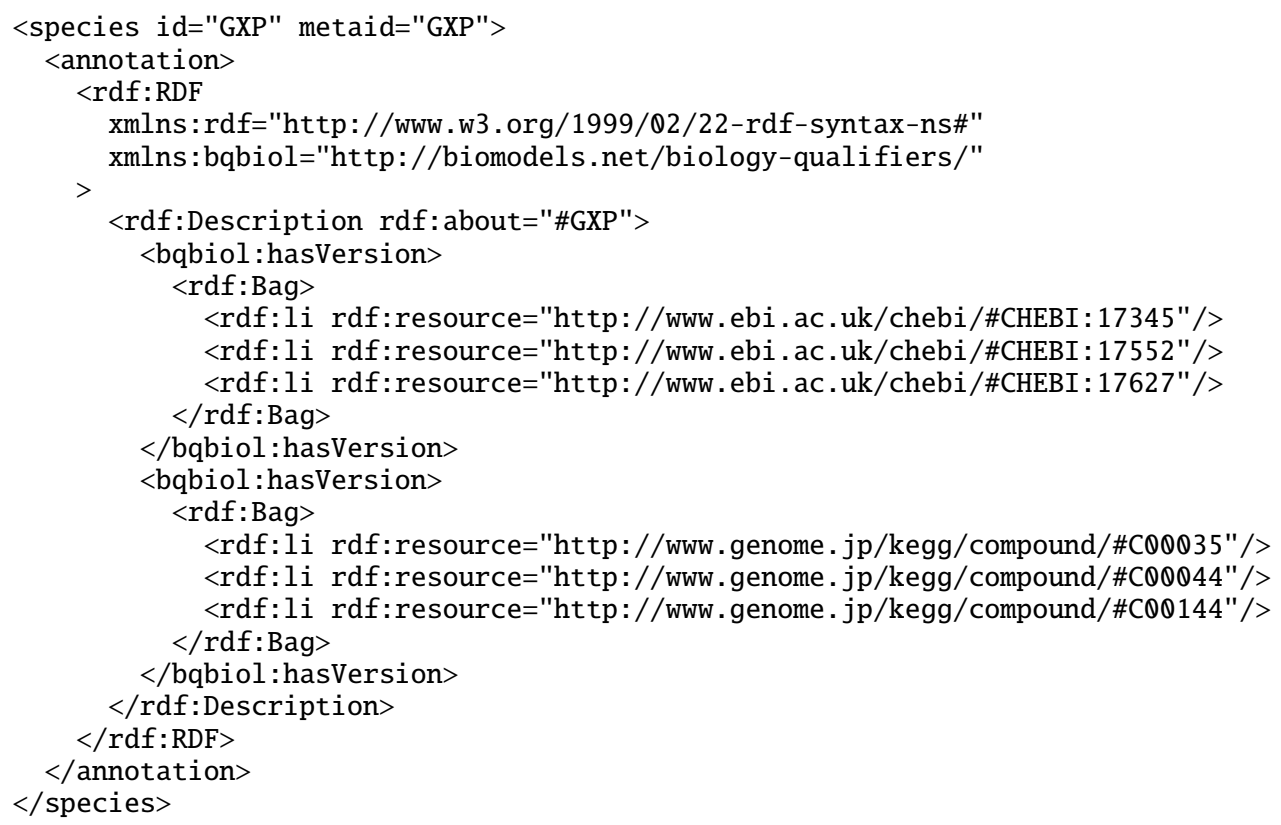

The following example presents a reaction being actually the combination of three different elementary molecular reactions. We annotate it with the three corresponding KEGG reactions, but also with the three corresponding enzymatic activities. Again the two hasPart elements represent two alternative annotations. The process represented by the Reaction structure is composed of three parts, and not six parts.

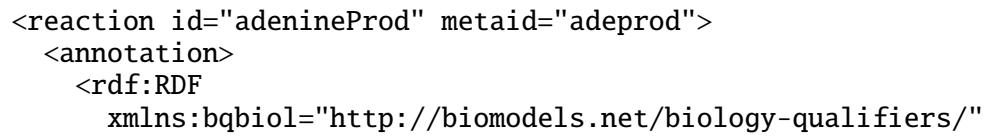




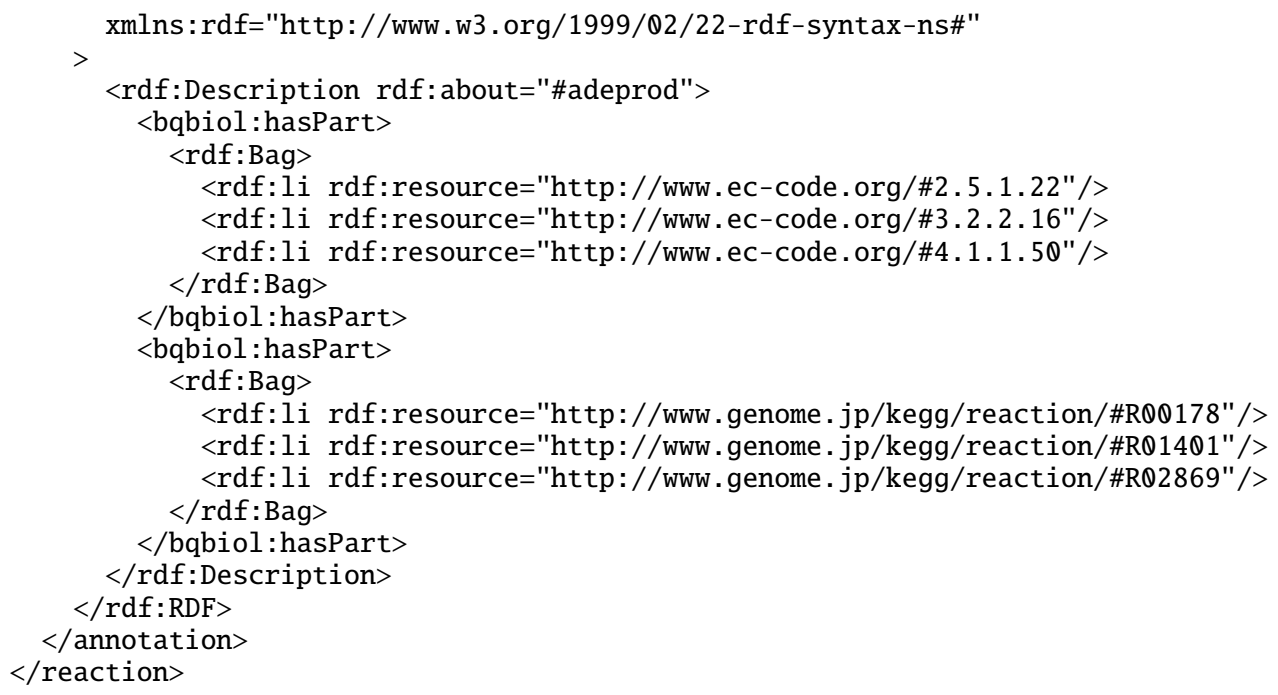

It is possible to mix different URIs in a given set. The following example presents two alternative annotations of the human hemoglobin, the first with ChEBI heme and the second with KEGG heme.

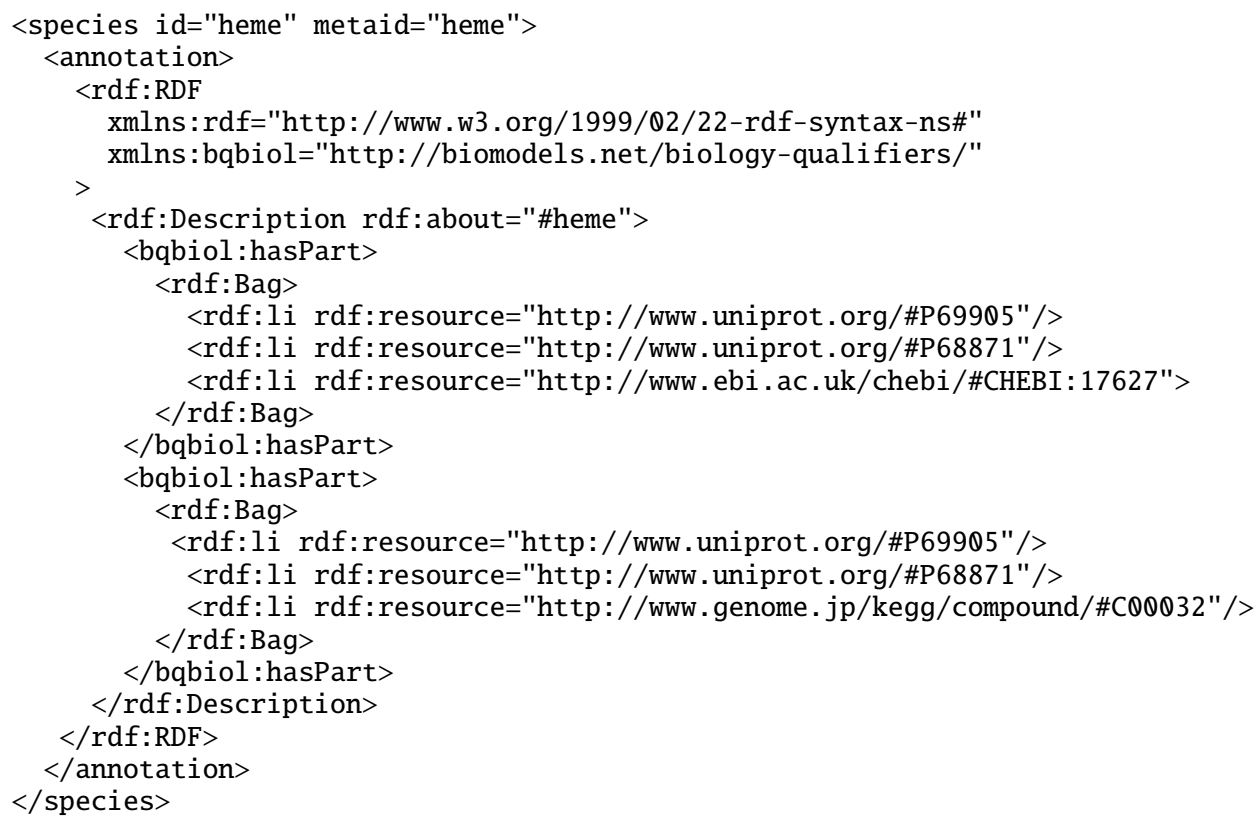

As formally defined above it is possible to use different qualifiers in the same annotation element. The following phosphorylation is annotated by its exact KEGG counterpart and by the generic GO term "phosphorylation".

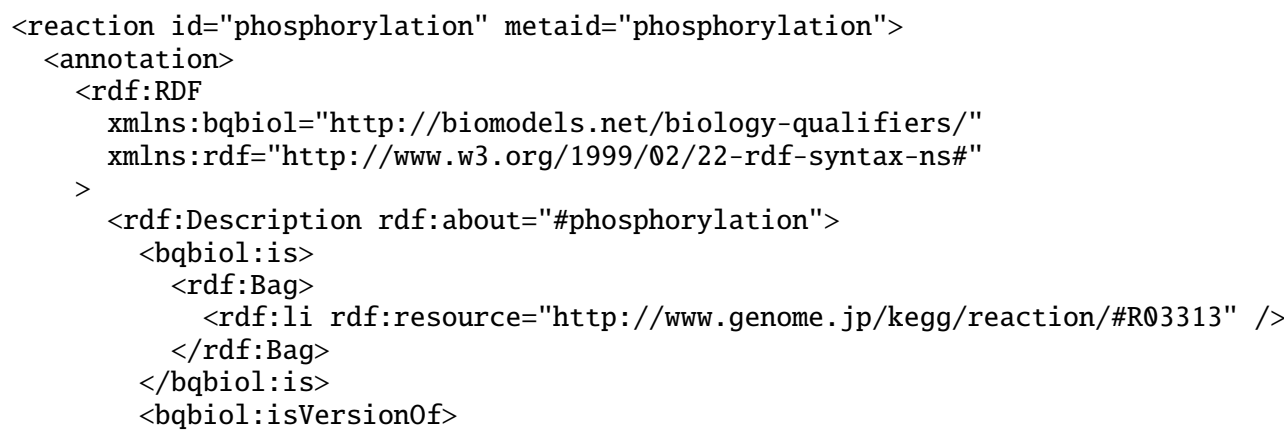




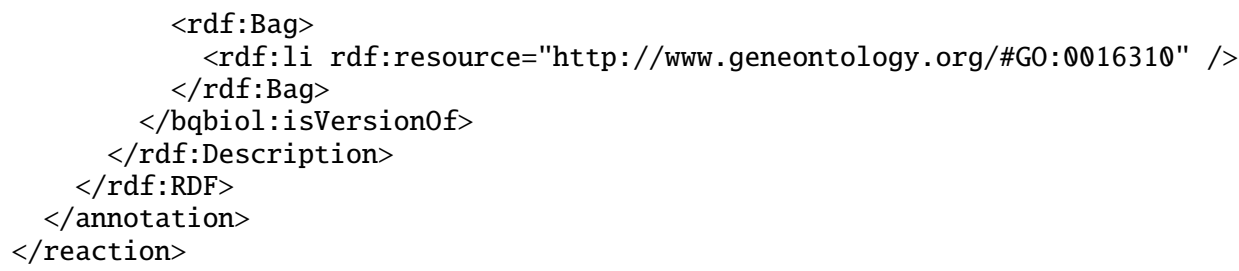




\section{Example models expressed in XML using SBML}

In this section, we present several examples of complete models encoded in XML using SBML Level 2.

\subsection{A simple example application of SBML}

Consider the following representation of an enzymatic reaction:

$$
E+S \underset{k_{\text {off }}}{\stackrel{k_{\text {on }}}{\longrightarrow}} E S \stackrel{k_{\text {cat }}}{\longrightarrow} E+P
$$

The following is the minimal SBML document encoding the model shown above:

$<$ ?xml version="1.0" encoding="UTF -8 "? >

<sbml level="2" version="3" xmlns="http://www.sbml.org/sbml/level2/version3"> $<$ model name="EnzymaticReaction">

$<$ list0fUnitDefinitions $>$

<unitDefinition id="per_second">

$<$ list0fUnits $>$

$<$ unit kind="second" exponent="-1"/>

$</$ list0fUnits $>$

$</$ unitDefinition $>$

<unitDefinition id="litre_per_mole_per_second">

$<$ list0fUnits $>$

$<$ unit kind="mole" exponent="-1"/>

$<$ unit kind="litre" exponent="1"/>

$<$ unit kind="second" exponent="-1"/>

$</$ list0fUnits $>$

$<$ /unitDefinition>

$</$ list0fUnitDefinitions $>$

$<$ list0fCompartments $>$

<compartment id="cytosol" size="1e-14"/>

$</$ list0fCompartments $>$

$<$ list0fSpecies $>$

<species compartment="cytosol" id="ES" initialAmount="0" name="ES"/>

<species compartment="cytosol" id="P" initialAmount=" 0 " name="P" $/>$

<species compartment="cytosol" id="S" initialAmount="1e-20" name="S"/>

<species compartment="cytosol" id="E" initialAmount="5e-21" name="E"/>

$</$ istOfSpecies $>$

$<$ list0fReactions $>$

$<$ reaction id="veq" >

$<$ list0fReactants $>$

<speciesReference species="E"/>

<speciesReference species="S"/>

$</$ list0fReactants $>$

$<$ list0fProducts $>$

<speciesReference species="ES"/>

$</$ listOfProducts $>$

$<$ kineticLaw $>$

<math xmlns="http://www.w3.org/1998/Math/MathML">

$<$ apply>

$<$ times/>

$<$ ci $>$ cytosol $</$ ci $>$

$<$ apply $>$

$<$ minus $/>$

$<$ apply $>$

$<$ times $/>$

$<$ ci $>$ kon $</$ ci $>$

$<\mathrm{Ci}>\mathrm{E}</ \mathrm{Ci}>$

$<\mathrm{ci}>\mathrm{S}</ \mathrm{ci}>$

$</$ apply $>$

$<$ apply>

$<$ times $/>$

$<\mathrm{ci}>\operatorname{koff}</ \mathrm{ci}>$

$</ a p p l y>$

$<\mathrm{ci}>\mathrm{ES}</ \mathrm{ci}>$

$</$ apply $>$

$</$ apply $>$

$</$ math $>$ 


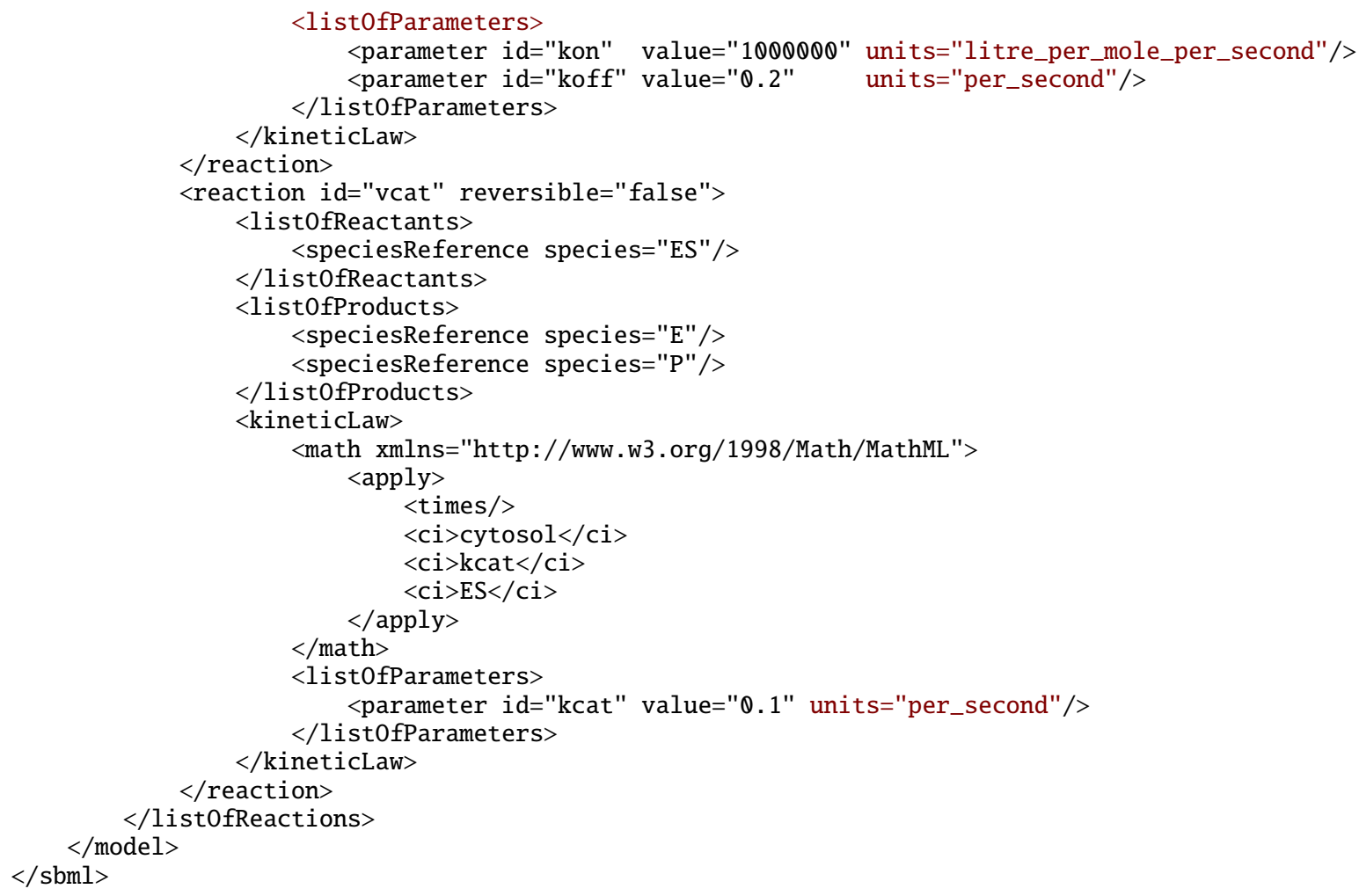

In this example, the model has the identifier "EnzymaticReaction". The model contains one compartment (with identifier "cytosol"), four species (with identifiers "ES", "P", "S", and "E"), and two reactions ("veq" and "vcat"). The elements in the list0fReactants and list0fProducts in each reaction refer to the names of elements listed in the list0fSpecies. The correspondences between the various elements is explicitly stated by the speciesReference elements.

The model also features local parameter definitions in each reaction. In this case, the three parameters ("kon", "koff", "kcat") all have unique identifiers and they could also have just as easily been declared global parameters in the model. Local parameters frequently become more useful in larger models, where it may become tedious to assign unique identifiers for all the different parameters.

\subsection{Example involving units}

The following model uses the units features of SBML Level 2. In this model, the default value of substance is changed to be mole units with a scale factor of -3 , or millimoles. This sets the default substance units in the model. The volume and time built-ins are left to their defaults, meaning volume is in litres and time is in seconds. The result is that, in this model, kinetic law formulas define rates in millimoles per second and the species identifiers in them represent concentration values in millimoles per litres. All the species elements set the initial amount of every given species to 1 millimole. The parameters "vm" and "km" are defined to be in millimoles per litres per second, and millimoles per litres, respectively.

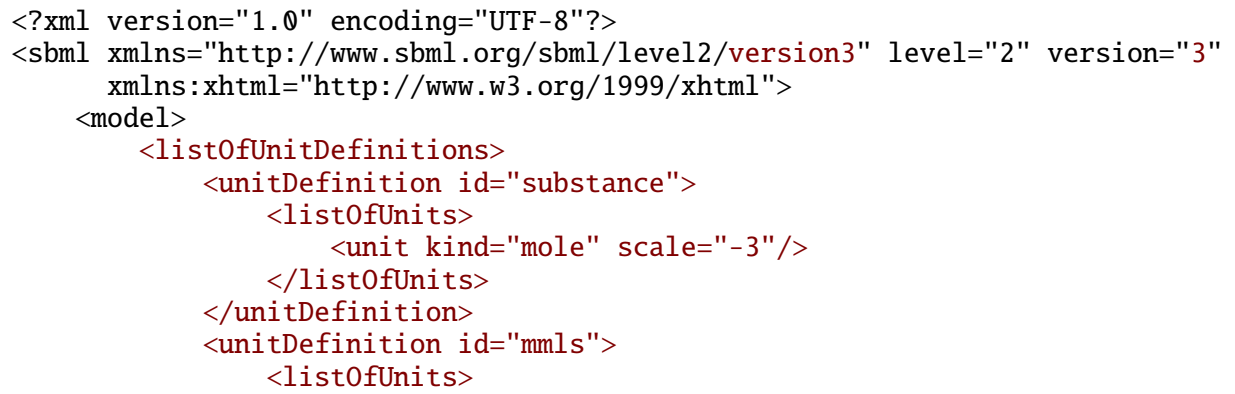




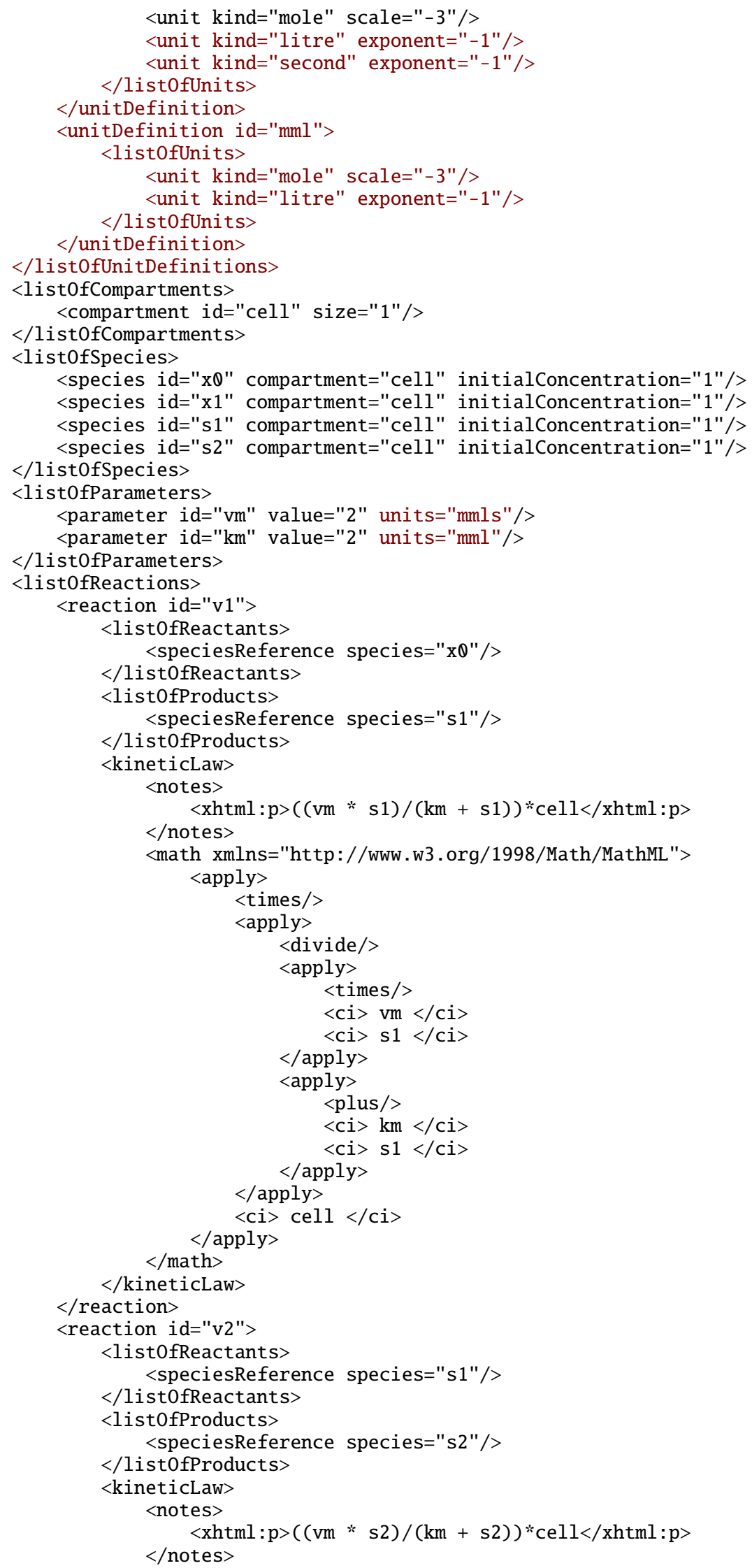


<math xmlns="http://www.w3.org/1998/Math/MathML">

$<$ apply>

$<$ times $/>$

$<$ apply $>$

$<$ divide/>

$<$ apply $>$

$<$ times $/>$

$<\mathrm{ci}>\mathrm{vm}</ \mathrm{ci}\rangle$

$<$ apply $>$

$<\mathrm{ci}>\mathrm{s} 2</ \mathrm{ci}>$

$<$ apply $>$

$<$ plus $/>$

$<$ ci $>\mathrm{km}</ \mathrm{ci}>$

$<$ /apply $>$

$</$ apply $>$

$<$ ci $>$ cell </ci $>$

$</$ math $>$

$</$ apply $>$

$</$ kineticLaw $>$

$</$ reaction $>$

$<$ reaction id="v3">

$<$ list0fReactants $>$

<speciesReference species="s2"/>

$</$ list0fReactants $>$

$<$ list0fProducts $>$

<speciesReference species="x1"/>

$</$ listOfProducts $>$

$<$ kineticLaw $>$

$<$ notes $>$

$<\mathrm{xhtml}: \mathrm{p}>((\mathrm{vm} * \mathrm{x} 1) /(\mathrm{km}+\mathrm{x} 1)) * \operatorname{cell}</ \mathrm{xhtml}: \mathrm{p}>$

$</$ notes $>$

$<$ math xmlns="http://www.w3.org/1998/Math/MathML"> $<$ apply>

$<$ times/>

$<$ apply $>$

$<$ divide/>

$<$ apply $>$

$<$ times $/>$

$<$ ci $>$ vm $</ \mathrm{ci}>$

$<\mathrm{ci}>\mathrm{x} 1</ \mathrm{ci}>$

$</$ apply $>$

$<$ apply $>$

$<$ plus $/>$

$<$ ci $>\mathrm{km}</ \mathrm{ci}>$

$</$ apply $>$

$\langle\mathrm{ci}>\mathrm{x} 1</ \mathrm{ci}>$

$<$ apply $>$

$<$ ci $>$ cell </ci>

$</$ apply $>$

$</$ appl

$</$ kineticLaw $>$

$</$ reaction $>$

$</$ model $>$

$</$ list0fReactions $>$

$</$ sbml $>$

\subsection{Example of a discrete version of a simple dimerization reaction}

(Model contributed by Darren J. Wilkinson, Newcastle University, Newcastle upon Tyne, UK.)

This example illustrates subtle differences between models formulated for use in a continuous simulation framework (e.g., using differential equations) and those intended for a discrete simulation framework. The model shown here is suitable for use with a discrete stochastic simulation algorithm of the sort developed by Gillespie (1977). In such an approach, species are described in terms of molecular counts and simulation proceeds by computing the probability of the time and identity of the next reaction, then updating the species amounts appropriately. 
The model involves a simple dimerization reaction for a protein named "P":

$$
2 P \leftrightarrow P_{2}
$$

The SBML representation is shown below. There are several important points to note. First, the species "P" and "P2" declare they are always in discrete amounts by using the flag hasOnlySubstanceUnits="true". This indicates that when the species identifiers appear in mathematical formulas, the units are substance, not the default of substance/size. A second point is that, as a result, the corresponding "kinetic law" formulas do not need volume corrections. In Gillespie's approach, the constants in the rate expressions (here, "c1" and "c2") contain a contribution from the kinetic constants of the reaction and the size of the compartment in which the reactions take place. Finally, it is worth noting the rate expression for the forward reaction is a second-order mass-action reaction, but it is the discrete formulation of such a reaction rate (Gillespie, 1977).

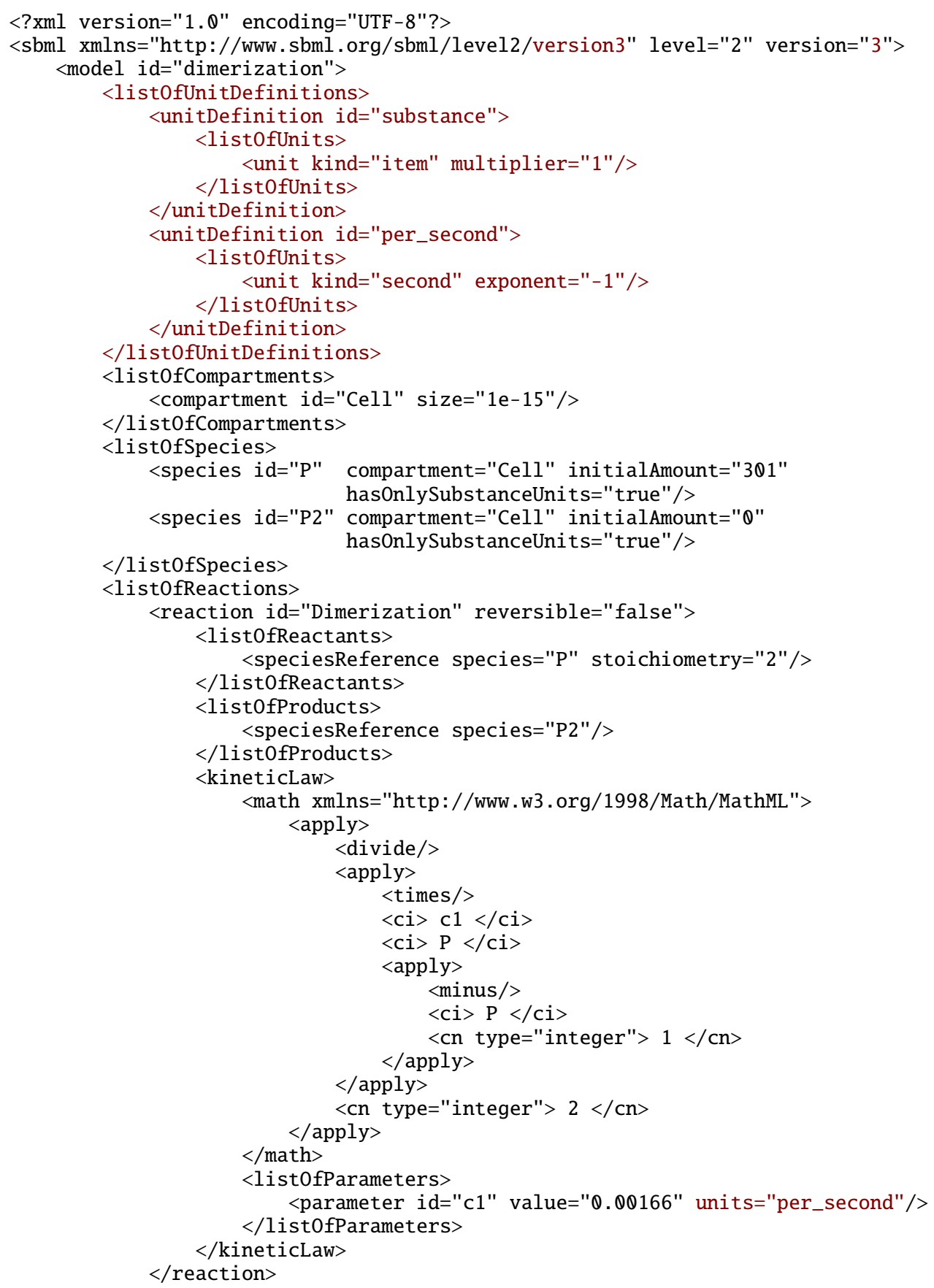


This example also illustrates the need to provide additional information in a model so that software tools using different mathematical frameworks can properly interpret it. In this case, a simulation tool designed for continuous ODE-based simulation would likely misinterpret the model (in particular the reaction rate formulas), unless it deduced that a discrete stochastic simulation was intended. One of the purposes of SBO annotations (Section 5) is to enable such interpretation without the need for deduction.

\subsection{Example involving assignment rules}

This section contains a model that simulates a system containing a fast reaction. This model uses rules to express the mathematics of the fast reaction explicitly rather than using the fast attribute on a reaction element. The system modeled is

$$
\begin{aligned}
& X_{0} \stackrel{k_{1} X_{0}}{\longrightarrow} \quad S_{1} \\
& S_{1} \stackrel{k_{f} S_{1}-k_{r} S_{2}}{\longrightarrow} S_{2} \\
& S_{2} \quad \stackrel{k_{2} S_{2}}{\longrightarrow} \quad X_{1} \\
& k_{1}=0.1, \quad k_{2}=0.15, \quad k_{f}=K_{e q} 10000, \quad k_{r}=10000, \quad K_{e q}=2.5 \text {. }
\end{aligned}
$$

where $X_{0}, S_{1}, S_{1}$, and $S_{2}$ are species in concentration units, and $k_{1}, k_{2}, k_{f}, k_{r}$, and $K_{e q}$ are parameters. This system of reactions can be approximated with the following new system:

$$
\begin{aligned}
& X_{0} \stackrel{k_{1} X_{0}}{\longrightarrow} T \\
& T \stackrel{k_{2} S_{2}}{\longrightarrow} X_{1} \\
& S_{1}=\frac{T}{1+K_{e q}} \\
& S_{2}=K_{e q} S_{1}
\end{aligned}
$$

where $T$ is a new species. The following example SBML model encodes the second system.

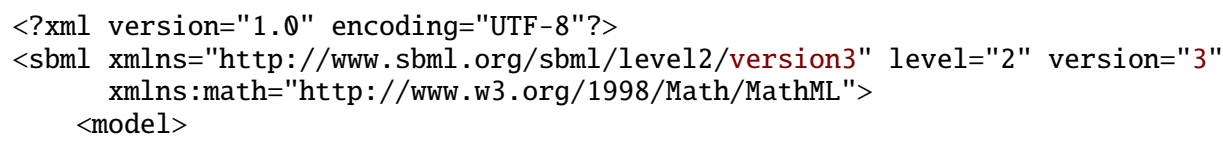




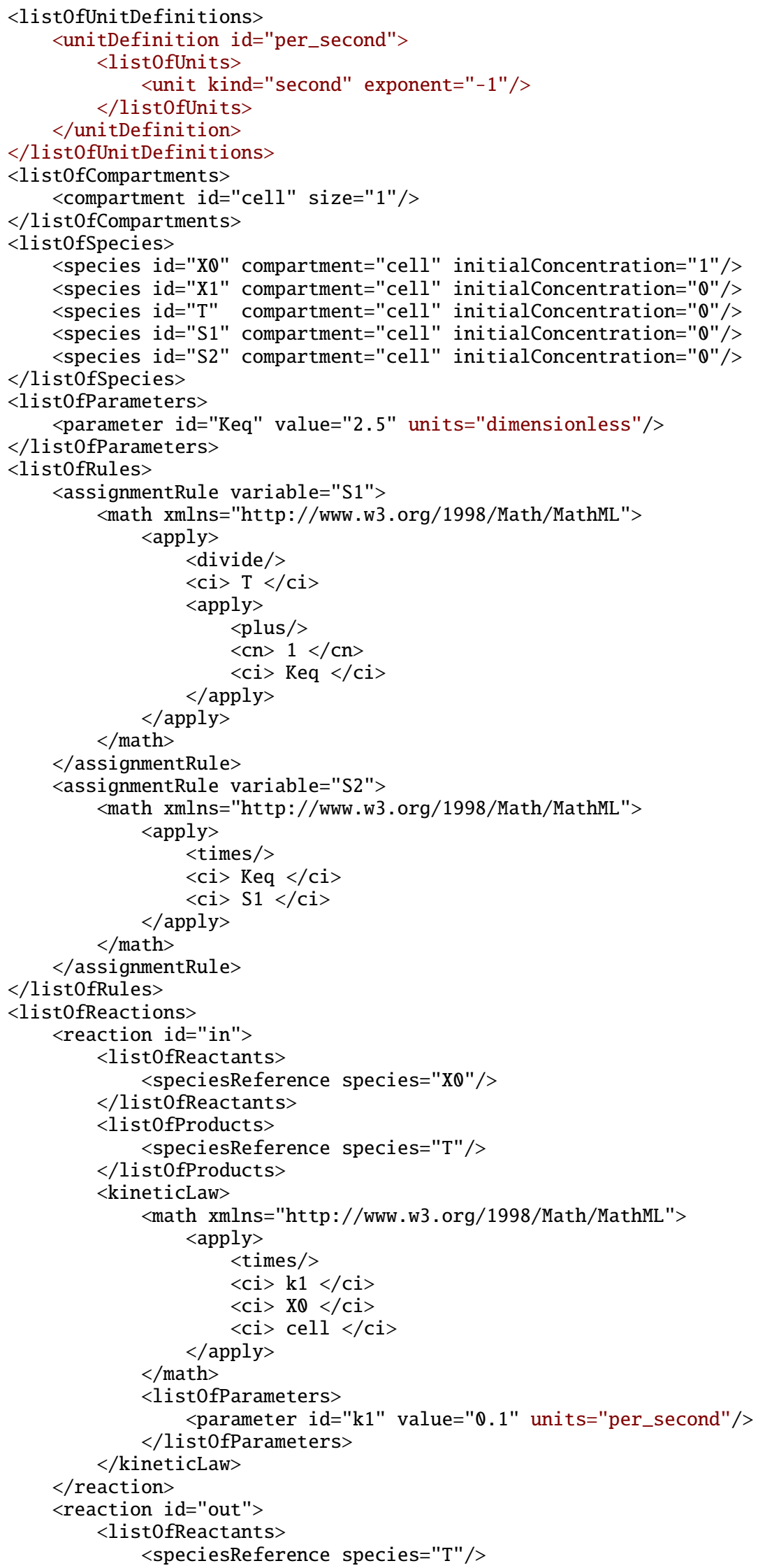




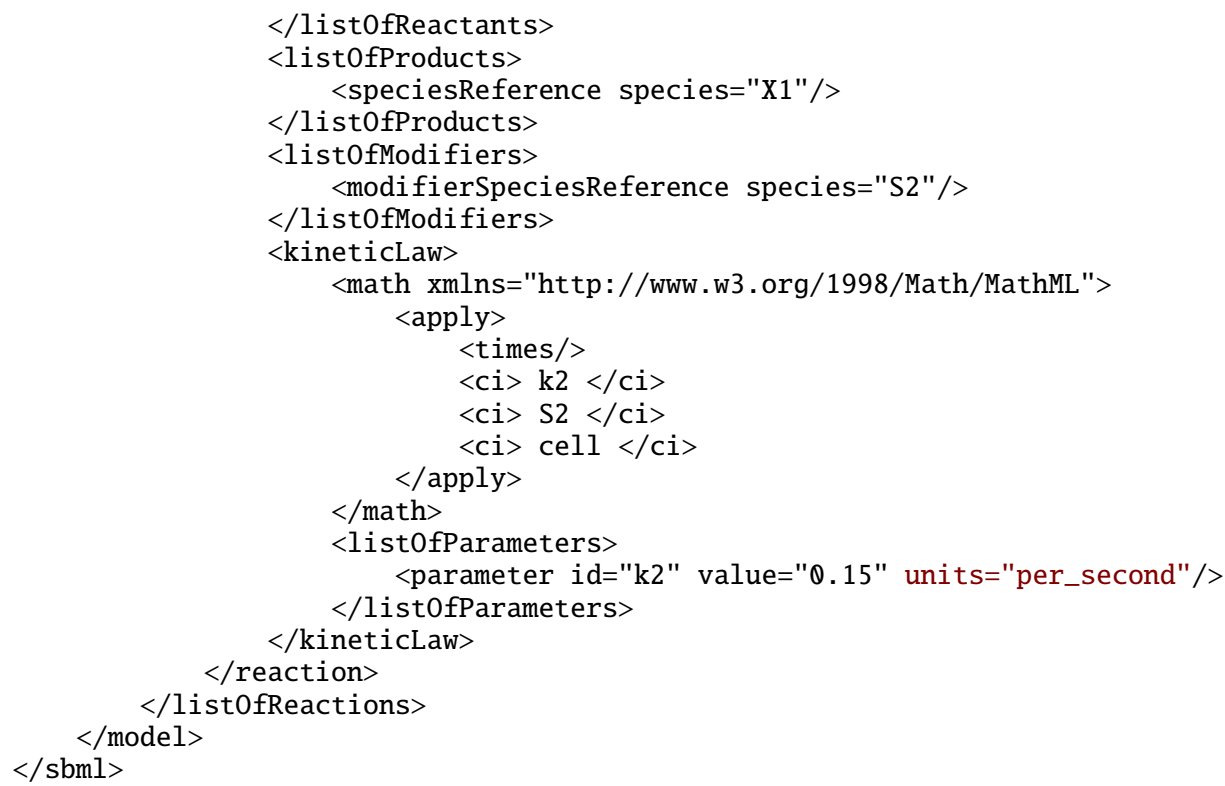

\subsection{Example involving algebraic rules}

This section contains an example model that contains an AlgebraicRule object. The model contains a different formulation of the fast reaction described in Section 7.4. The system described in Section 7.4 can be approximated with the following system:

$$
\begin{aligned}
& X_{0} \stackrel{k_{1} X_{0}}{\longrightarrow} T \\
& T \stackrel{k_{2} S_{1}}{\longrightarrow} X_{1} \\
& S_{2}=K_{e q} S_{1}
\end{aligned}
$$

with the constraint:

$$
S_{1}+S_{2}-T=0
$$

The following example SBML model encodes this approximate form.

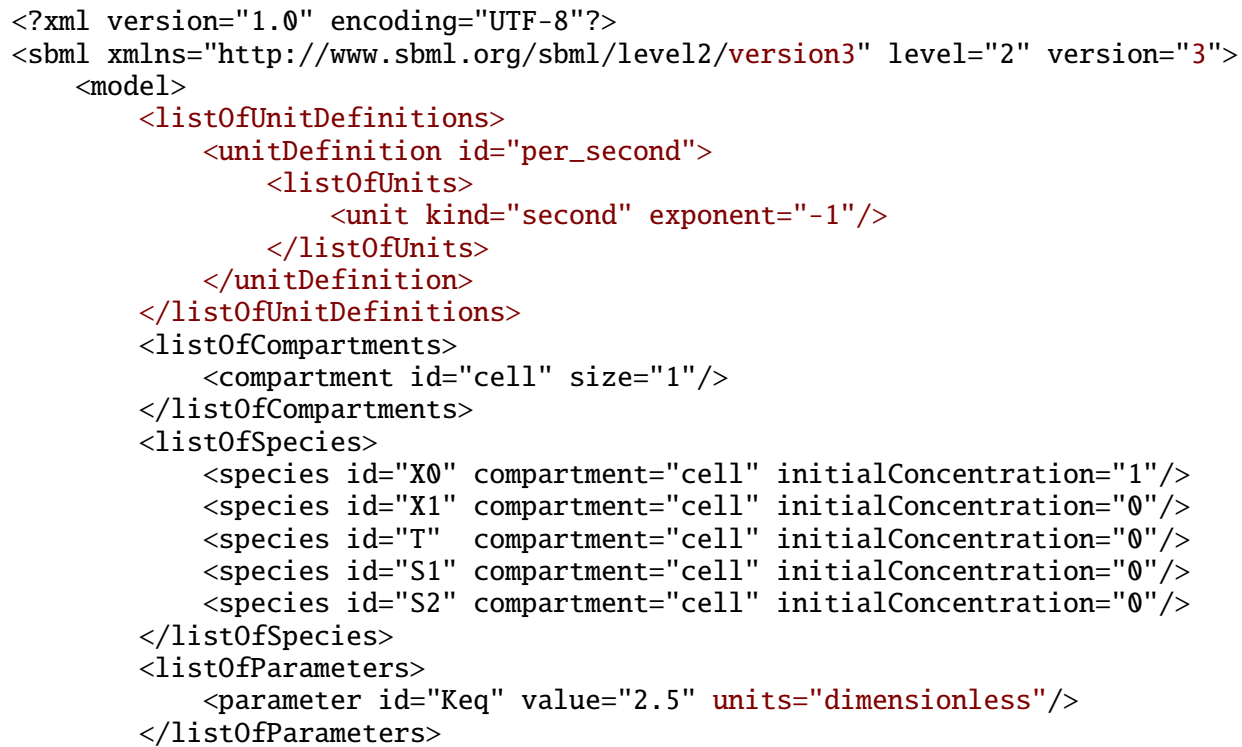




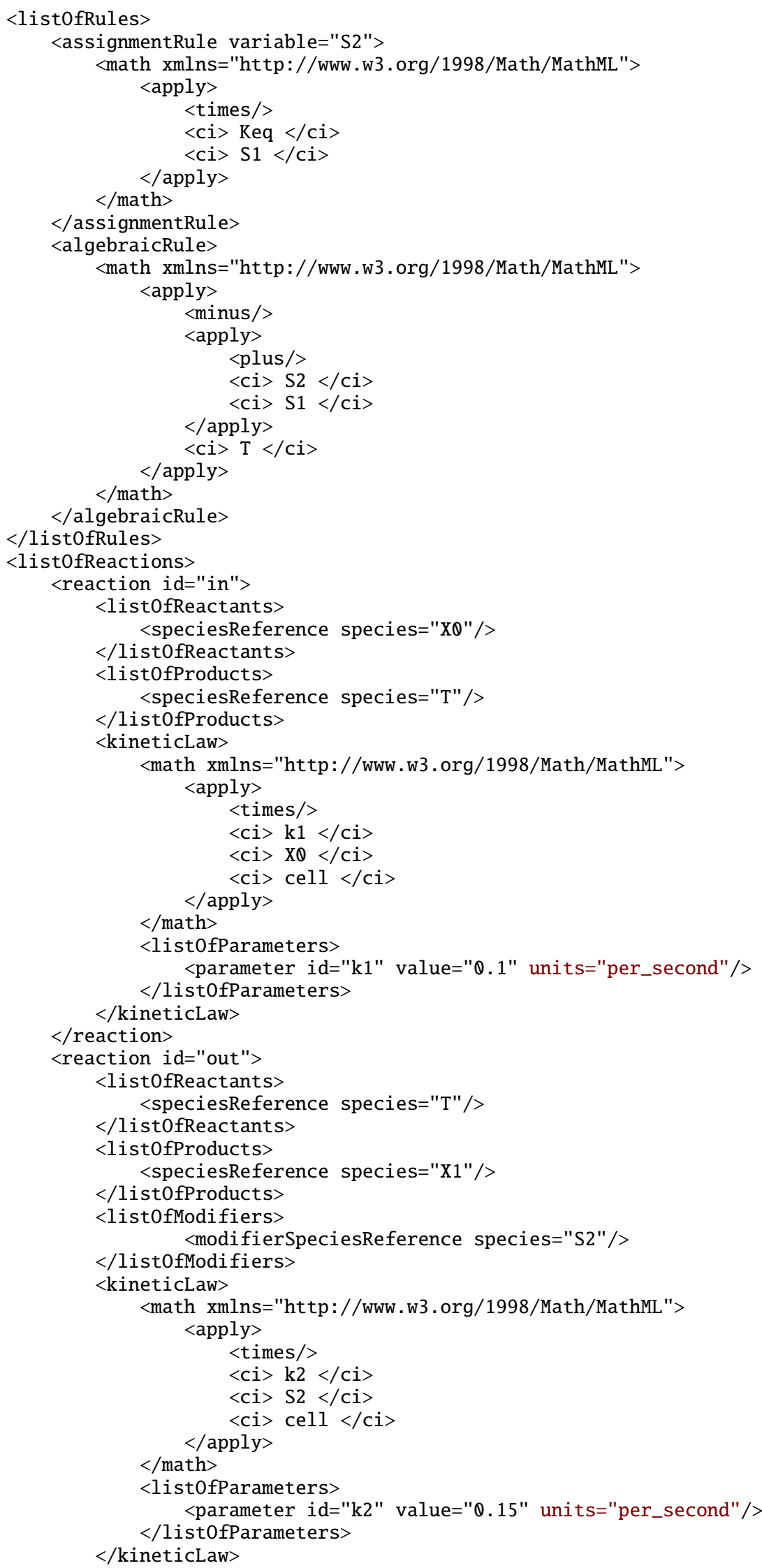




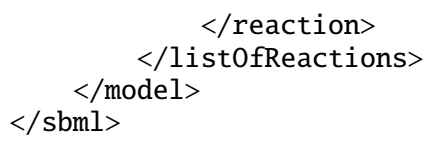

\subsection{Example with combinations of boundaryCondition and constant values on Species with RateRule objects}

In this section, we discuss a model that includes four species, each with a different combination of values for their boundaryCondition and constant attributes. The model represents a hypothetical system containing one reaction,

$$
S_{1}+S_{2} \stackrel{k_{1} S_{1} S_{2} S_{3}}{\longrightarrow} S_{4}
$$

where $S_{3}$ is a species that catalyzes the conversion of species $S_{1}$ and $S_{2}$ into $S_{4} . S_{1}$ and $S_{2}$ are on the boundary of the system (i.e., $S_{1}$ and $S_{2}$ are reactants but their values are not determined by a kinetic law). The value of $S_{1}$ in the system is determined over time by the rate rule:

$$
\frac{d S_{1}}{d t}=k_{2}
$$

The values of constant parameters in the system are:

$$
S_{2}=1, \quad S_{3}=2, \quad k_{1}=0.5, \quad k_{2}=0.1
$$

and the initial values of species are:

$$
S_{1}=0, \quad S_{4}=0
$$

The value of $S_{1}$ varies over time so in SBML $S_{1}$ has a constant attribute with a default value of "false". The values of $S_{2}$ and $S_{3}$ are fixed so in SBML they have a constant attribute values of "true". $S_{3}$ only occurs as a modifier so the value of its boundaryCondition attribute can default to "false". $S_{4}$ is a product whose value is determined by a kinetic law and therefore in the SBML representation has "false" (the default) for both its boundaryCondition and constant attributes.

The following is the SBML rendition of the model shown above:

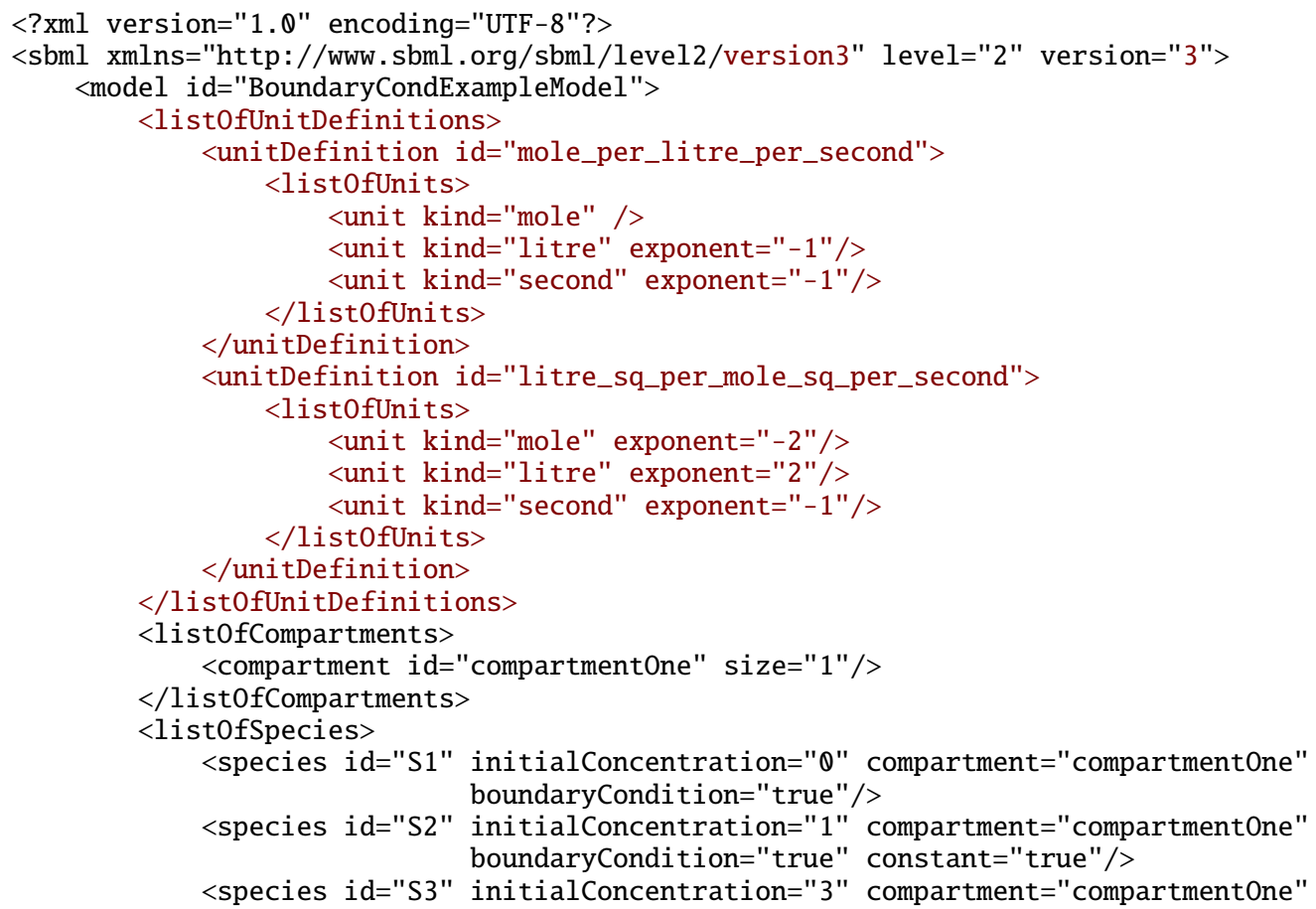


constant="true"/>

<species id="S4" initialConcentration="ब" compartment="compartmentOne"/>

$</$ listOfSpecies $>$

$<$ list0fParameters $>$

$<$ parameter id="k1" value="0.5" units="litre_sq_per_mole_sq_per_second"/>

$<$ parameter id="k2" value="0.1" units="mole_per_litre_per_second"/>

$</$ list0fParameters $>$

$<$ list0fRules $>$

$<$ rateRule variable="S1">

$<$ math xmlns="http://www.w3.org/1998/Math/MathML">

$</$ math $>$

$<\mathrm{ci}>\mathrm{k} 2</ \mathrm{ci}>$

$</$ rateRule $>$

$</$ listofRules $>$

$<$ list0fReactions $>$

<reaction id="reaction_1" reversible="false">

$<$ list0fReactants $>$

$<$ speciesReference species="S1"/>

<speciesReference species="S2"/>

$</$ list0fReactants $>$

$<$ list0fProducts $>$

$<$ speciesReference species="S4"/>

$</$ ist0fProducts $>$

$<$ list0fModifiers $>$

$<$ modifierSpeciesReference species="S3"/>

$</$ list0fModifiers $>$

$<$ kineticLaw $>$

$<$ math xmlns="http://www.w3.org/1998/Math/MathML">

$<$ apply $>$

$<$ times $/>$

$<$ ci $>\mathrm{k} 1</ \mathrm{ci}>$

$<$ ci $>$ S $1</$ ci $>$

$<$ ci $>$ S2 $</$ ci $>$

$<$ ci $>$ S3 $</$ ci $>$

$<$ ci $>$ compartment0ne $</$ ci $>$

$</$ math $>$

$</$ apply $>$

$</$ kineticLaw $>$

$</$ reaction $>$

$</$ model $>$

$</$ list0fReactions $>$

$</$ sbml $>$

\subsection{Example of translation from a multi-compartmental model to ODEs}

This section contains a model with 2 compartments and 4 reactions. The model is derived from LotkaVolterra, with the addition of a reversible transport step. When observed in a time-course simulation, three of this model's species display damped oscillations.

$$
\begin{aligned}
& \text { cytosol } \\
& \begin{array}{l}
\text { nucleus } \\
\quad X+Y_{1 n} \stackrel{k_{1}}{\longrightarrow} 2 Y_{1 n} \stackrel{K_{\mathrm{T}}}{\rightleftharpoons} 2 Y_{1 c}+2 Y_{2} \stackrel{k_{2}}{\longrightarrow} 4 Y_{2} \stackrel{k_{3}}{\longrightarrow} \emptyset
\end{array}
\end{aligned}
$$

Figure 29: A example multi-compartmental model.

Figure 29 illustrates the arrangement of compartments and reactions in the model LotkaVolterra_tranport. The text of the SBML representation of the model is shown below, and it is followed by its complete translation into ordinary differential equations. In this SBML model, the reaction equations are in substance per time units. The reactions have also been simplified to reduce common stoichiometric factors. The species variables are in concentration units; their initial quantities are declared using the attribute initialAmount on the species definitions, but since the attribute hasOnlySubstanceUnits is not set to true, the identifiers of 
the species represent their concentrations when those identifiers appear in mathematical expressions elsewhere in the model. Note that the species whose identifier is " $\mathrm{X}$ " is a boundary condition, as indicated by the attribute boundaryCondition="true" in its definition. The attribute speciesType="Y" in the definitions of "Y1n" and "Y1c" indicates that these species are pools of the same participant, but located in different compartments.

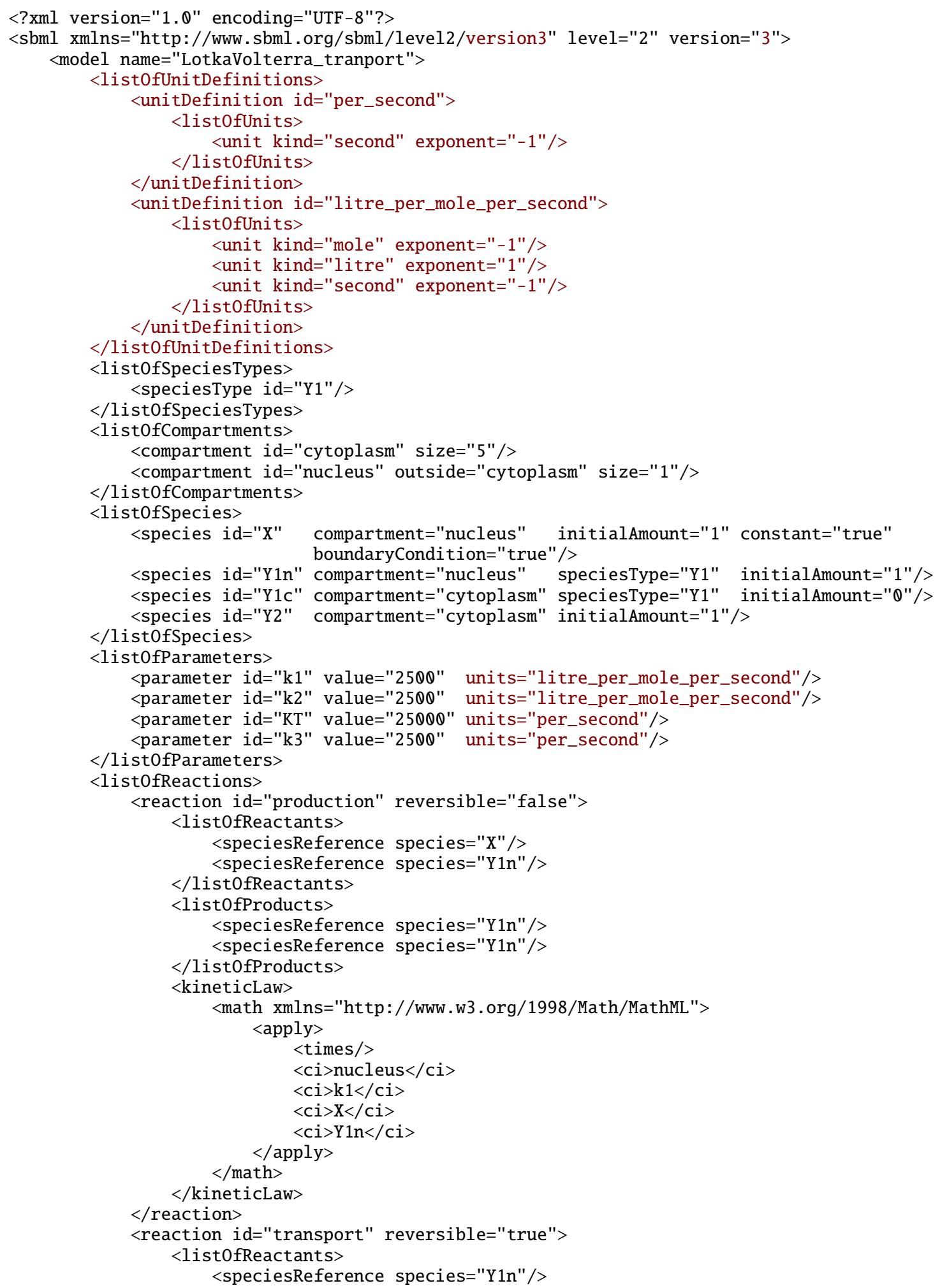




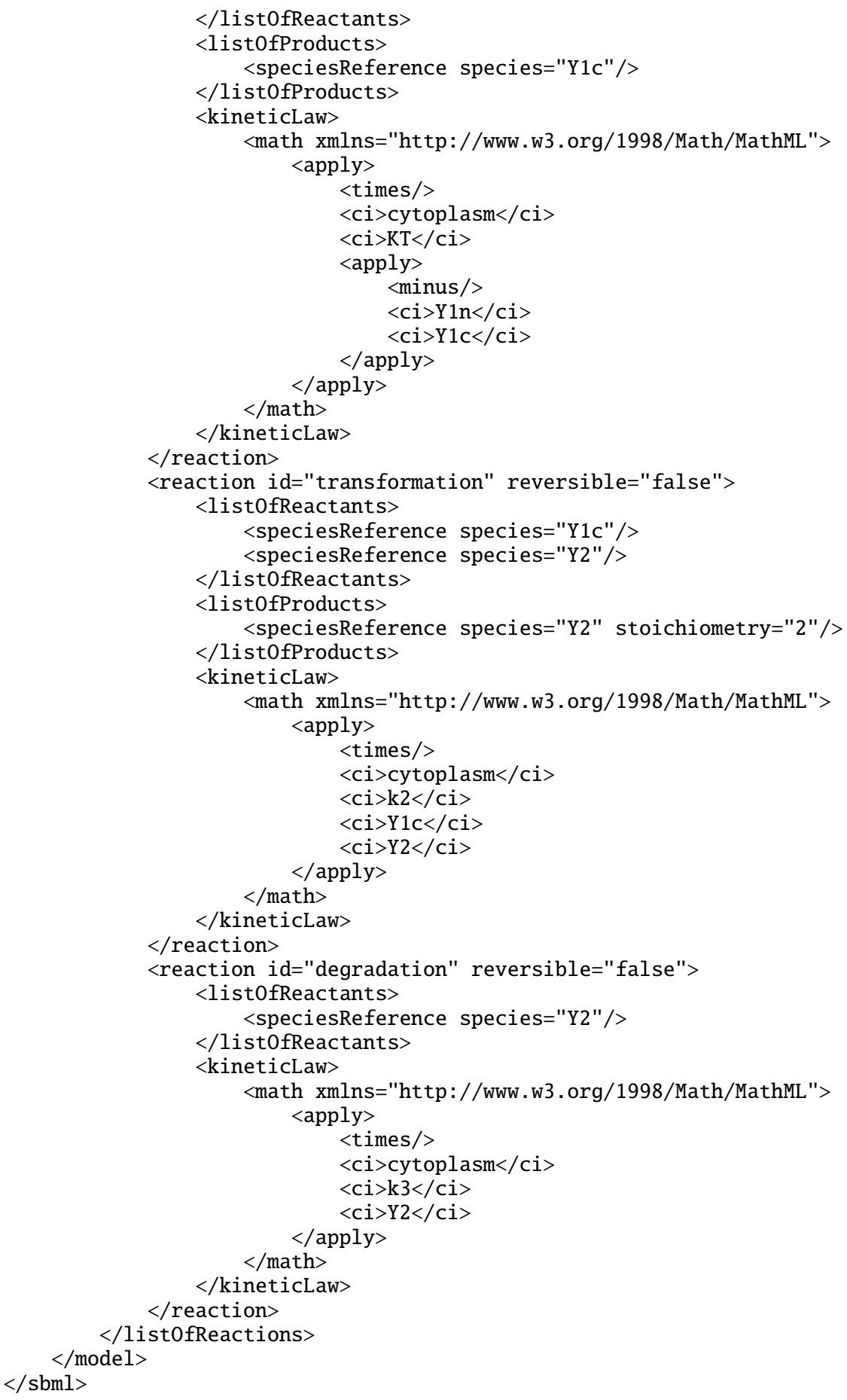

The ODE translation of this model is as follows. First, we give the values of the constant parameters:

$$
k_{1}=2500, \quad k_{2}=2500, \quad K_{3}=25000, \quad K_{T}=2500
$$

Next, here are the initial conditions of the variables. The species variables $X, Y_{1 n}, Y_{1 c}$, and $Y_{2}$ in the following equations are all in terms of concentrations, and we use $V_{n}$ to represent for the size of compartment "nucleus" and $V_{c}$ to represent for the size of compartment "cytoplasm":

$$
V_{n}=1, \quad V_{c}=5, \quad X=1, \quad Y_{1 n}=1, \quad Y_{1 c}=0, \quad Y_{2}=1
$$


And finally, here are the differential equations:

$$
\begin{array}{rlrl}
\frac{d X}{d t} & =0 & \\
V_{n} \frac{d Y_{1 n}}{d t} & =k_{1} \cdot X \cdot Y_{1 n} \cdot V_{n}-K_{T} \cdot\left(Y_{1 n}-Y_{1 c}\right) \cdot V_{c} & & \text { reactions production and transport } \\
V_{c} \frac{d Y_{1 c}}{d t} & =K_{T} \cdot\left(Y_{1 n}-Y_{1 c}\right) \cdot V_{c}-k_{2} \cdot Y_{1 c} \cdot Y_{2} \cdot V_{c} & & \text { reactions transport and transformation } \\
V_{c} \frac{d Y_{2}}{d t} & =k_{2} \cdot Y_{1 c} \cdot Y_{2} \cdot V_{c}-k_{3} \cdot Y_{2} \cdot V_{c} & & \text { reactions transformation and degradation }
\end{array}
$$

As formulated here, this example assumes constant volumes. If the sizes of the compartments "cytoplasm" or "nucleus" could change during simulation, then it would be preferable to use a different approach to constructing the differential equations. In this alternative approach, the ODEs would compute substance change rather than concentration change, and the concentration values would be computed using separate equations. This approach is used in Section 4.13.6.

\subsection{Example involving function definitions}

This section contains a model that uses the function definition feature of SBML. Consider the following hypothetical system:

$$
S_{1} \stackrel{f\left(S_{1}\right)}{\longrightarrow} S_{2}
$$

where

$$
f(x)=2 \times x
$$

The following is the XML document that encodes the model shown above:

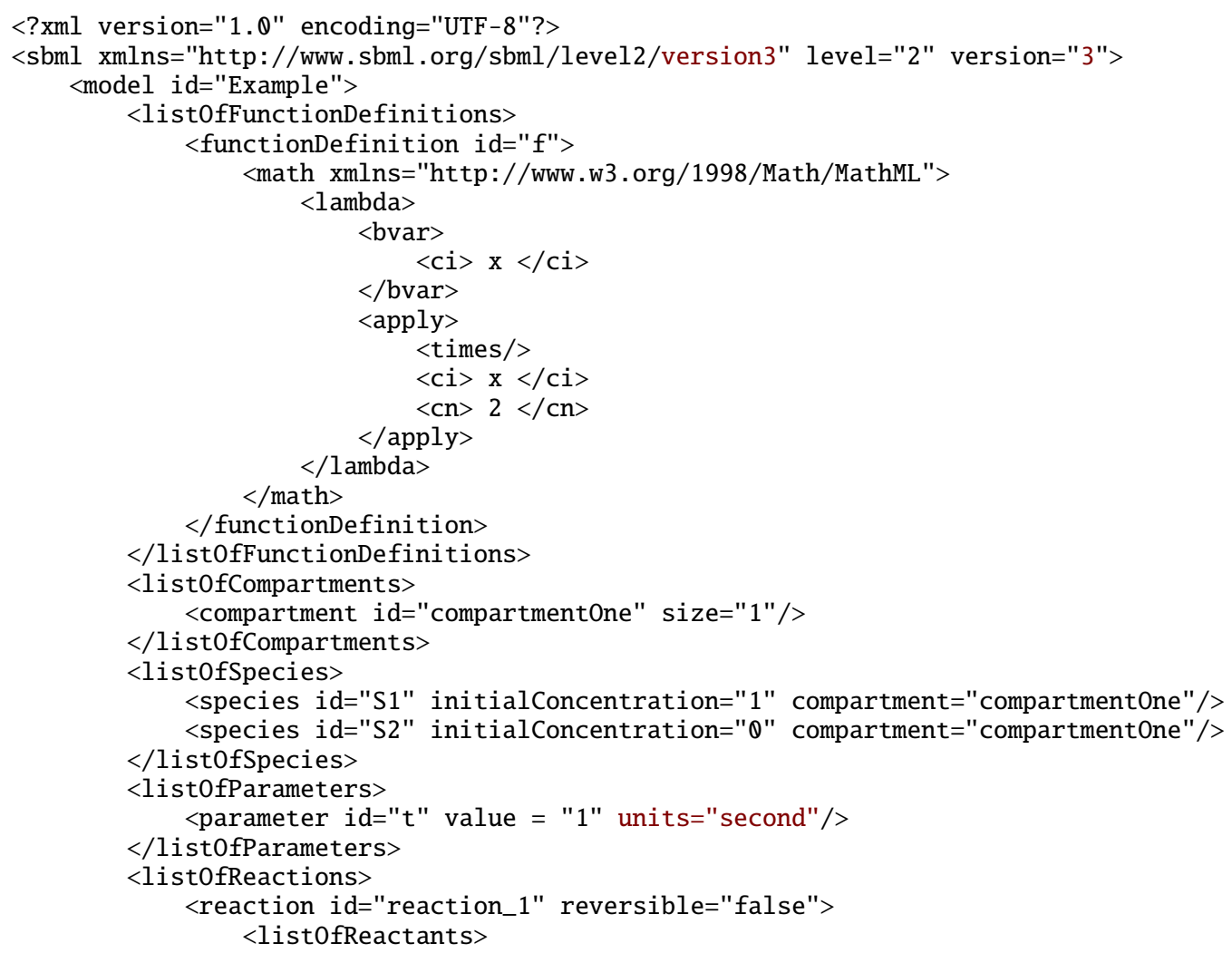




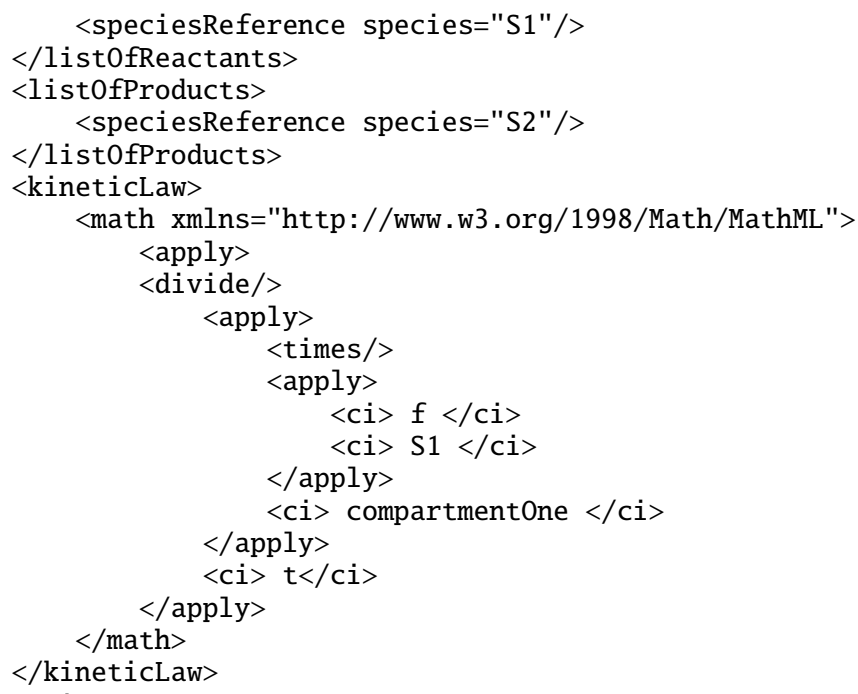

\subsection{Example involving delay functions}

The following is a simple model illustrating the use of delay to represent a gene that suppresses its own expression. The model can be expressed in a single rule:

$$
\frac{d P}{d t}=\frac{\frac{1}{1+m\left(P_{\text {delayed }}\right)^{q}}-P}{\tau}
$$

where

$$
\begin{aligned}
P_{\text {delayed }} & \text { is } \operatorname{delay}\left(P, \Delta_{t}\right) \text { or } \mathrm{P} \text { at } t-\Delta_{t} \\
P & \text { is protein concentration } \\
\tau & \text { is the response time } \\
m & \text { is a multiplier or equilibrium constant } \\
q & \text { is the Hill coefficient }
\end{aligned}
$$

and the species quantities are in concentration units. The text of an SBML encoding of this model is given below:

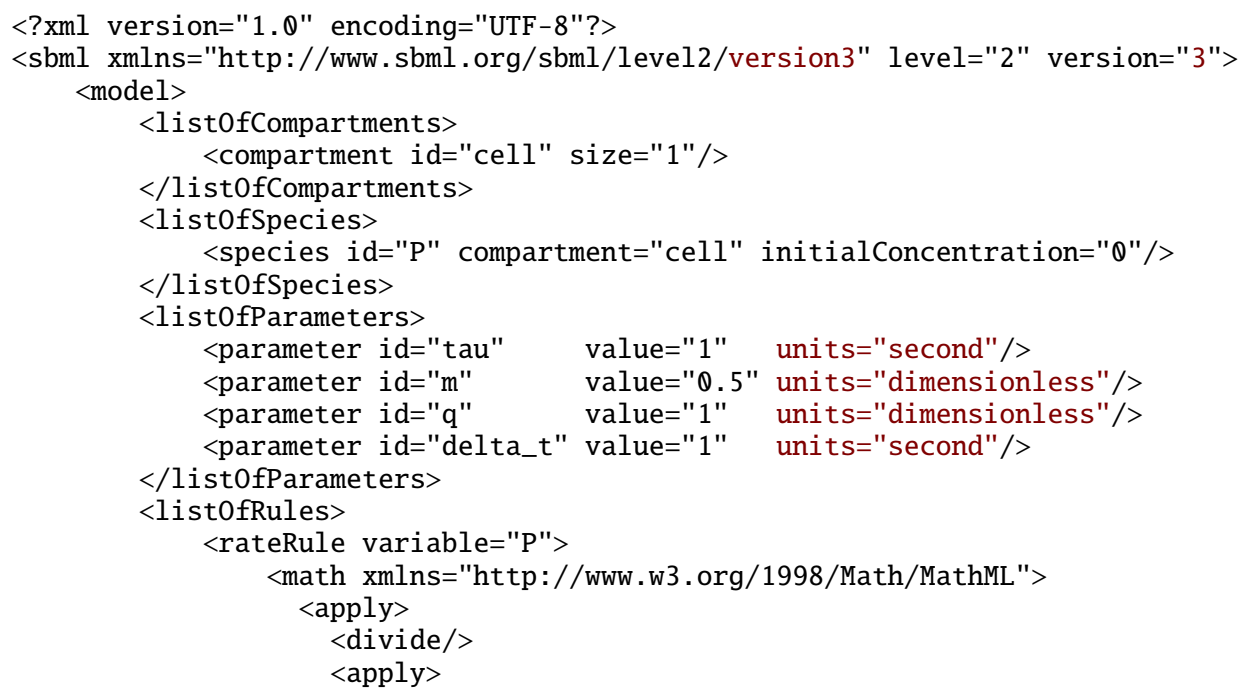




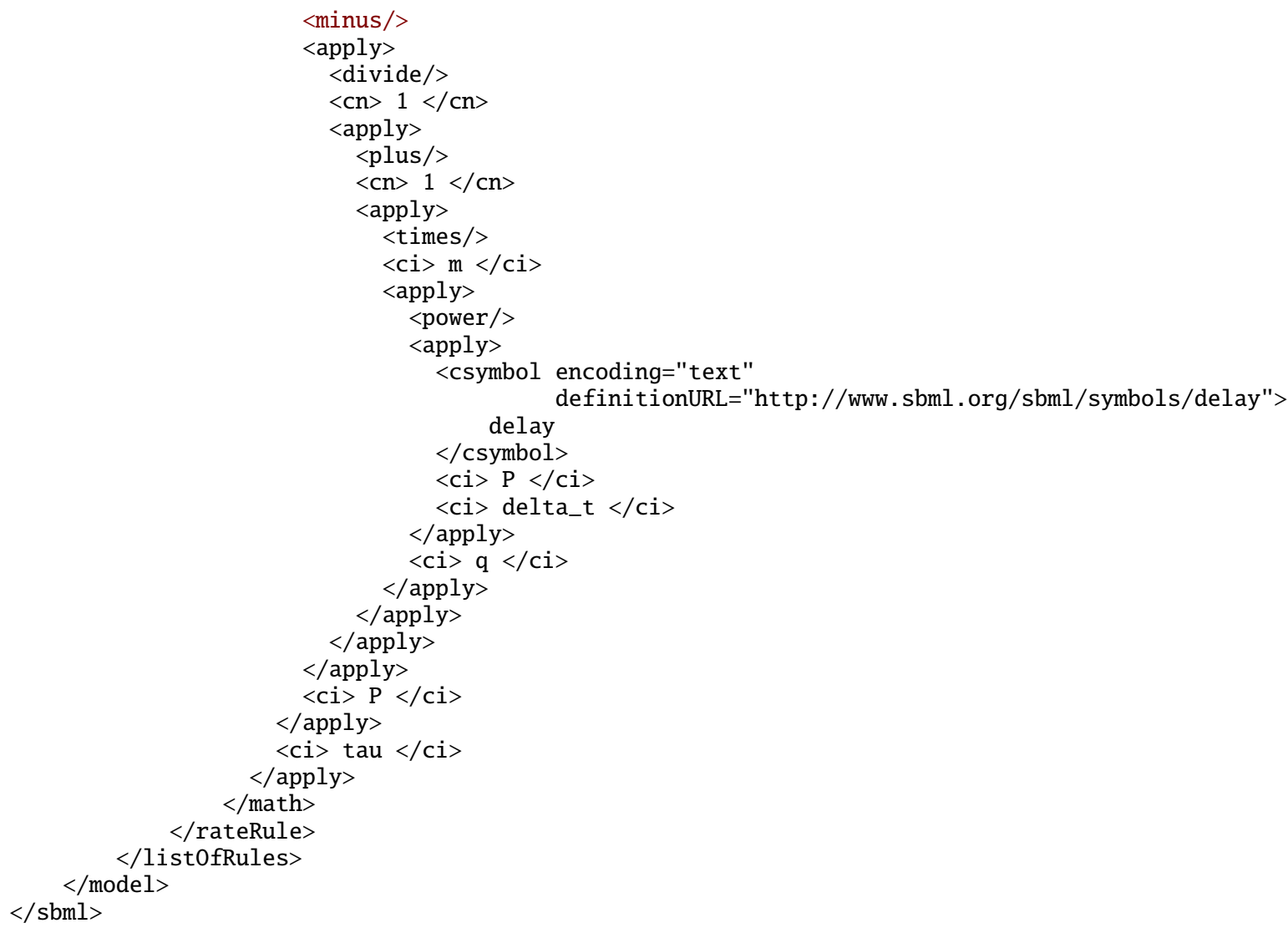

\subsection{Example involving events}

This section presents a simple model system that demonstrates the use of events in SBML. Consider a system with two genes, $G_{1}$ and $G_{2}$. $G_{1}$ is initially on and $G_{2}$ is initially off. When turned on, the two genes lead to the production of two products, $P_{1}$ and $P_{2}$, respectively, at a fixed rate. When $P_{1}$ reaches a given concentration, $G_{2}$ switches on. This system can be represented mathematically as follows:

$$
\begin{aligned}
\frac{d P_{1}}{d t} & =k_{1}\left(G_{1}-P_{1}\right) \\
\frac{d P_{2}}{d t} & =k_{2}\left(G_{2}-P_{2}\right) \\
G_{2} & = \begin{cases}0 & \text { when } P_{1} \leq \tau, \\
1 & \text { when } P_{1}>\tau .\end{cases}
\end{aligned}
$$

The initial values are:

$$
G_{1}=1, \quad G_{2}=0, \quad \tau=0.25, \quad P_{1}=0, \quad P_{2}=0, \quad k_{1}=k_{2}=1 .
$$

The SBML Level 2 representation of this as follows:

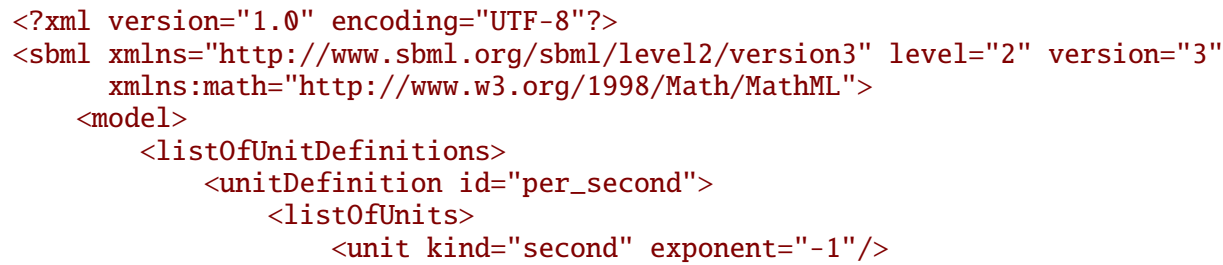




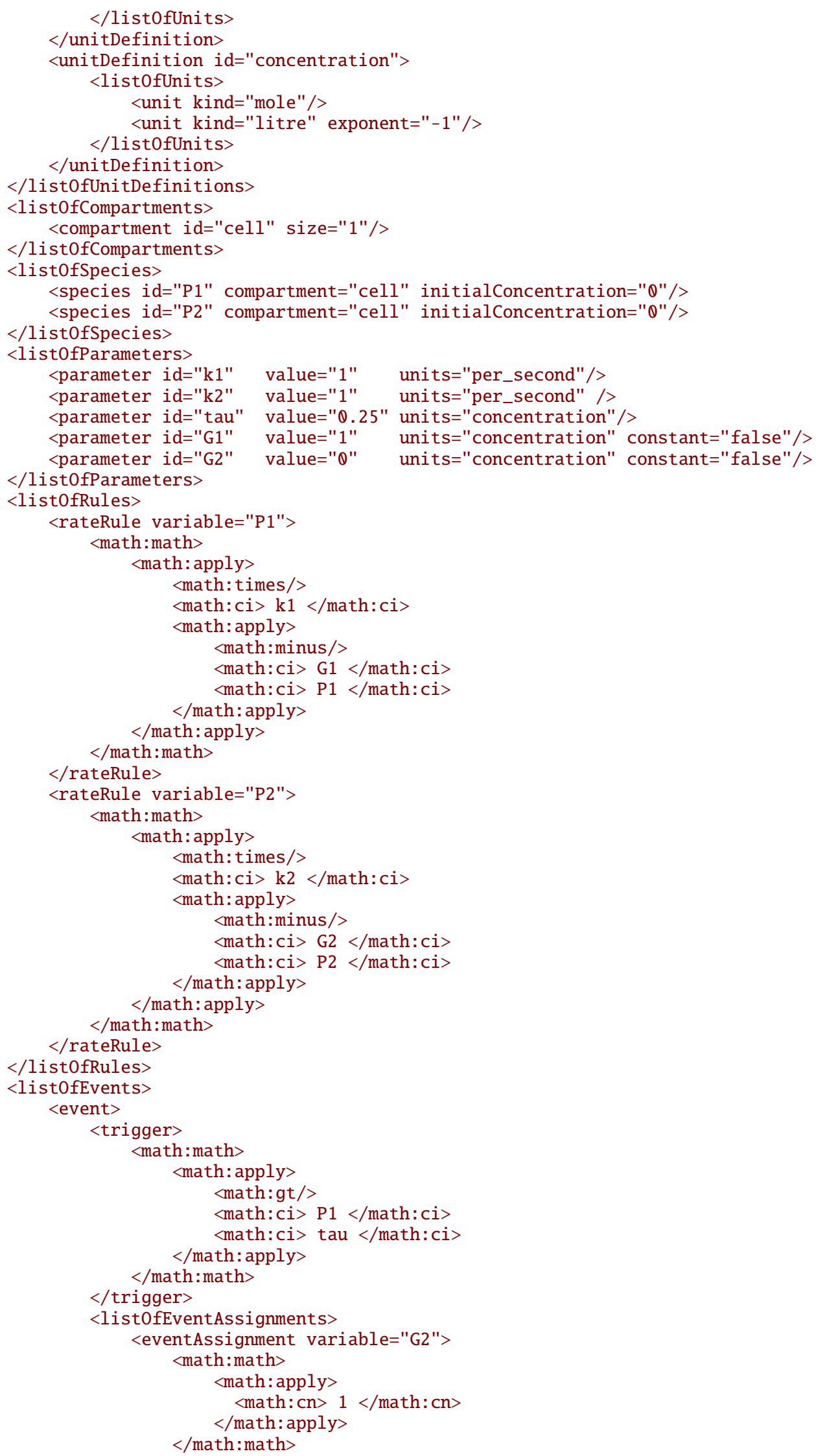




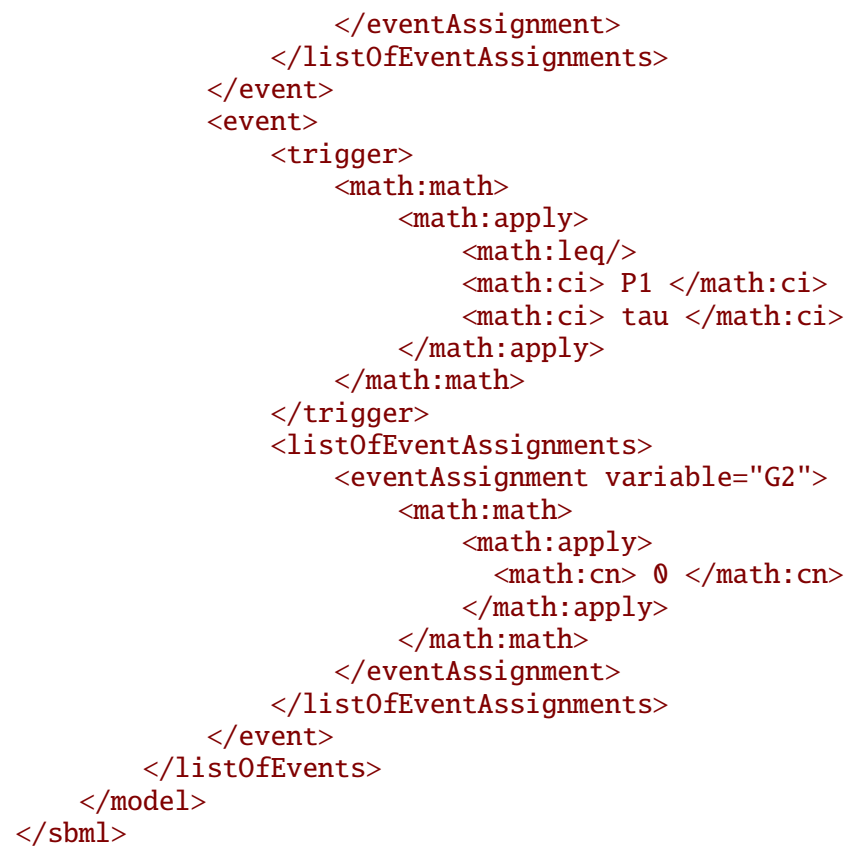

\subsection{Example involving two-dimensional compartments}

The following example is a model that uses a two-dimensional compartment. It is a fragment of a larger model of calcium regulation across the plasma membrane of a cell. The model includes a calcium influx channel, "Ca_channel", and a calcium-extruding PMCA pump, "Ca_Pump". It also includes two cytosolic proteins that buffer calcium via the "CalciumCalbindin_gt_BoundCytosol" and "CalciumBuffer_gt_BoundCytosol" reactions. Finally, the rate expressions in this model do not include explicit factors of the compartment volumes; instead, the various rate constants are assume to include any necessary corrections for volume.

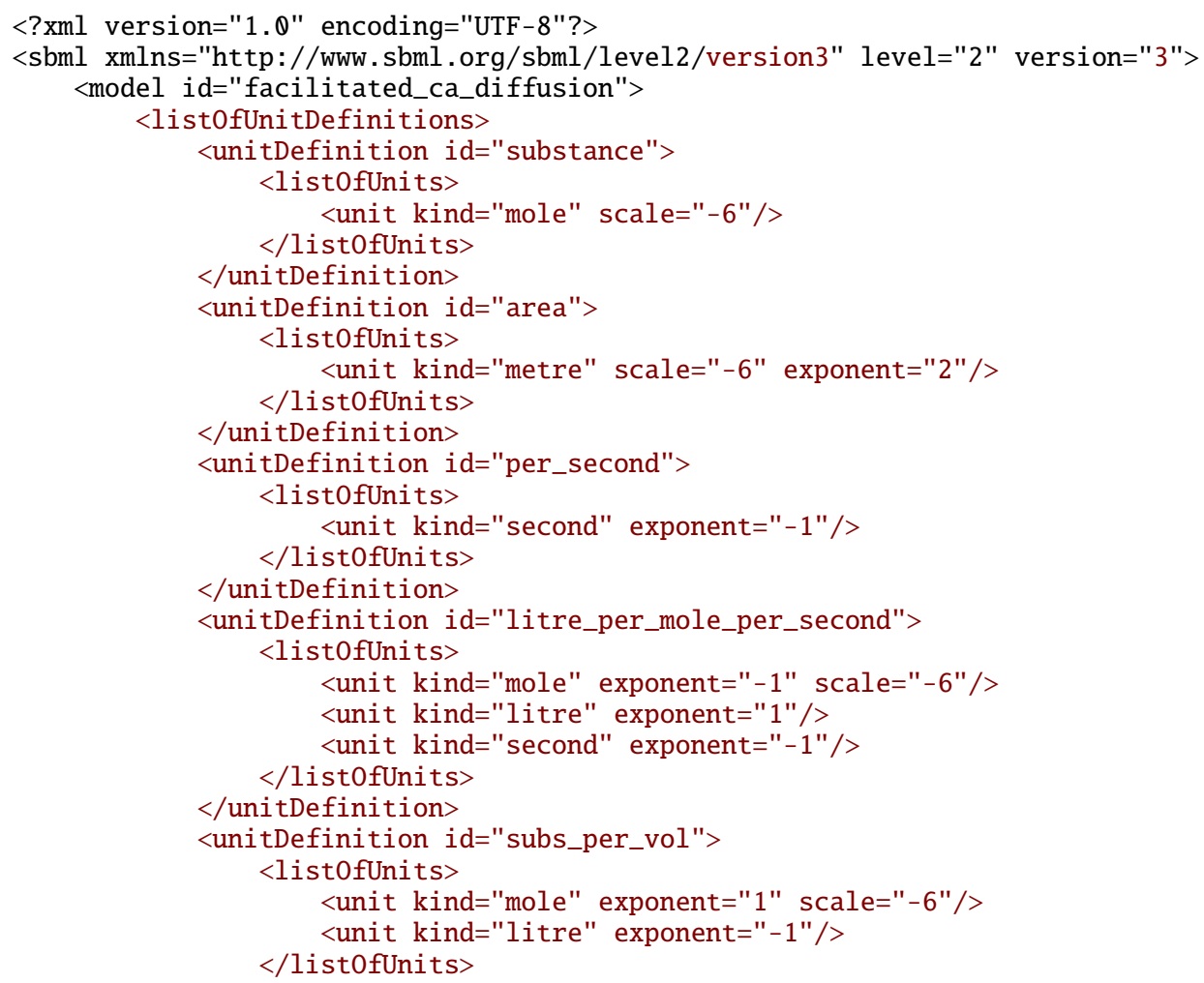




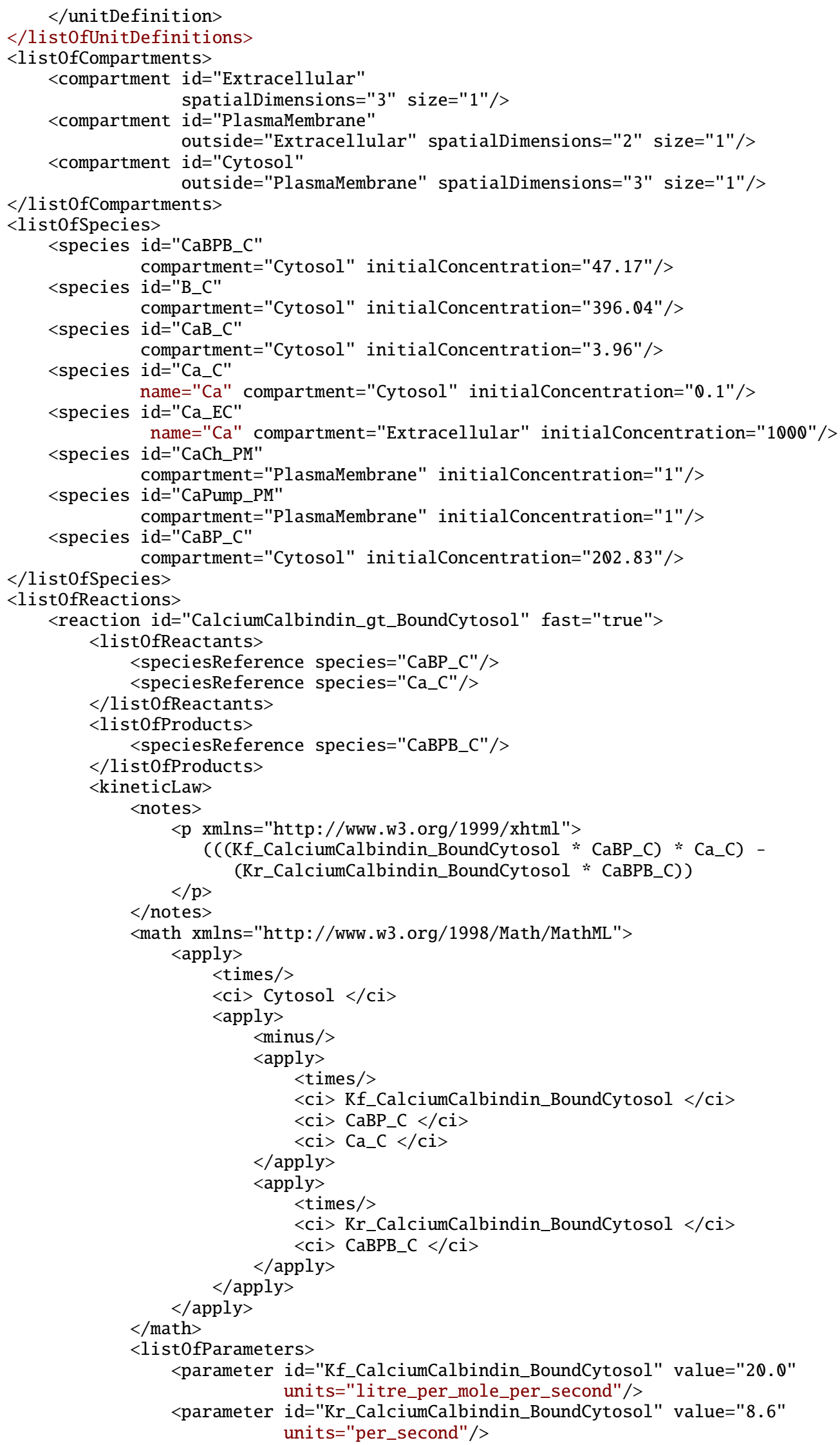




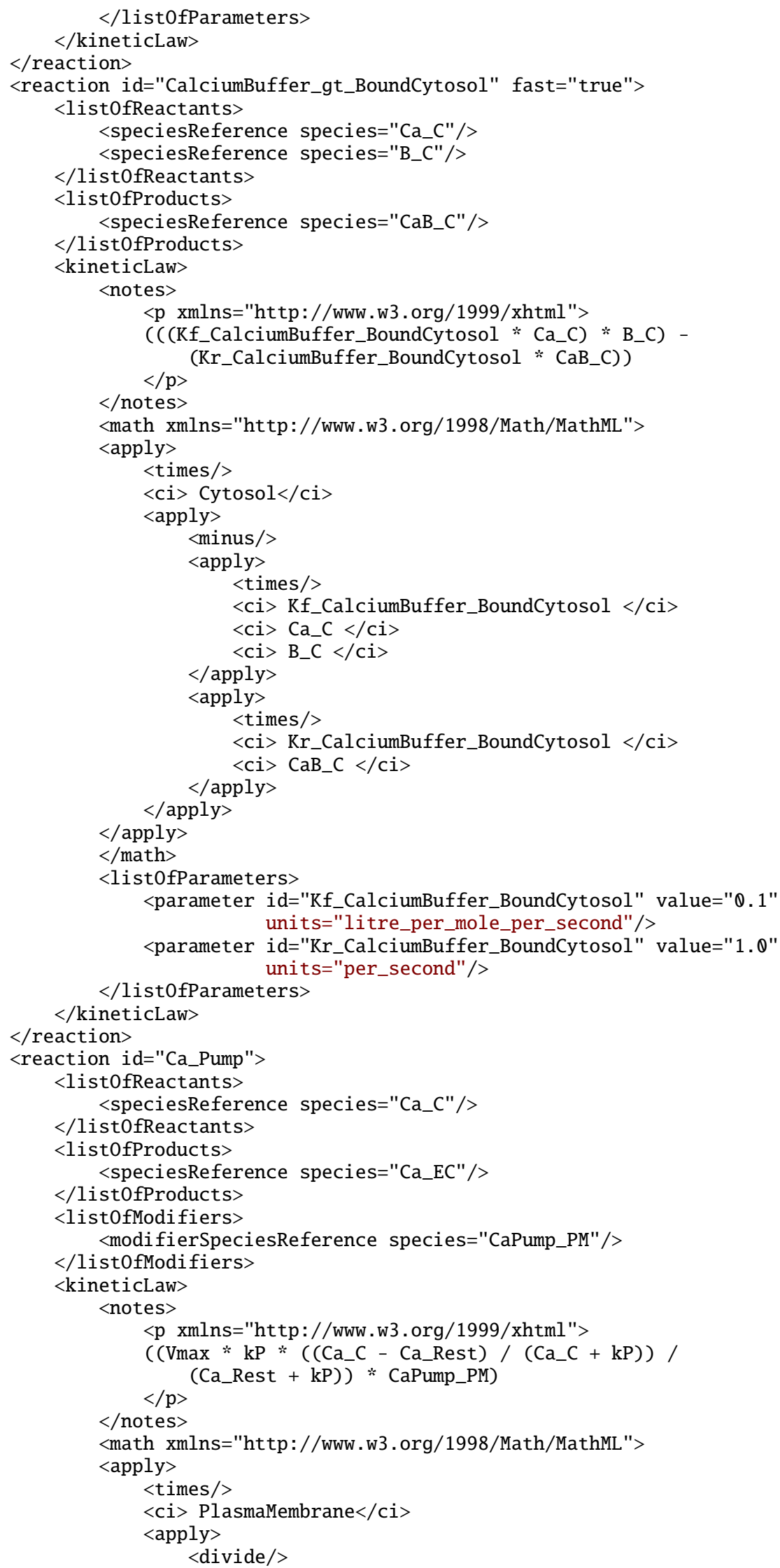




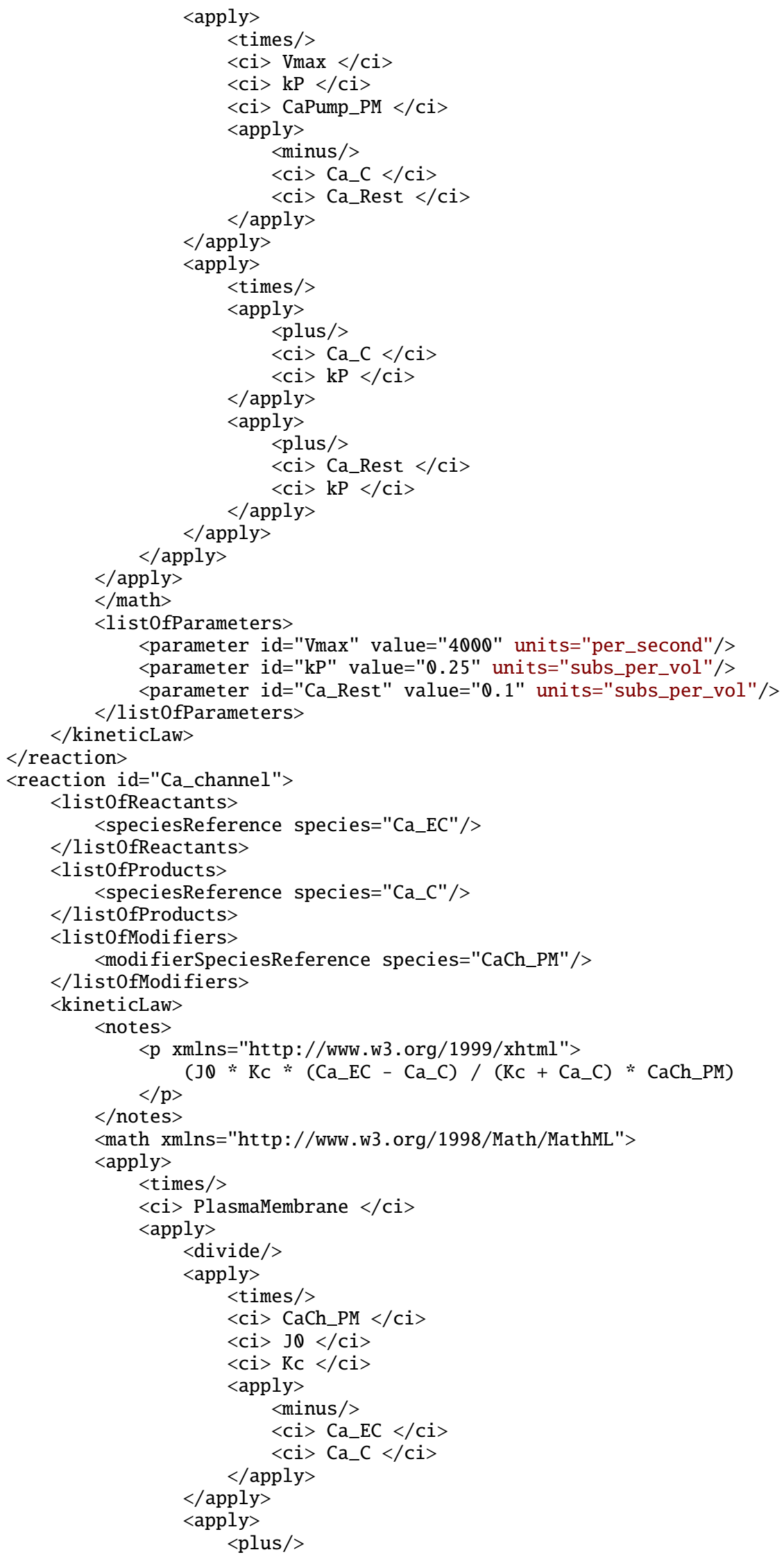




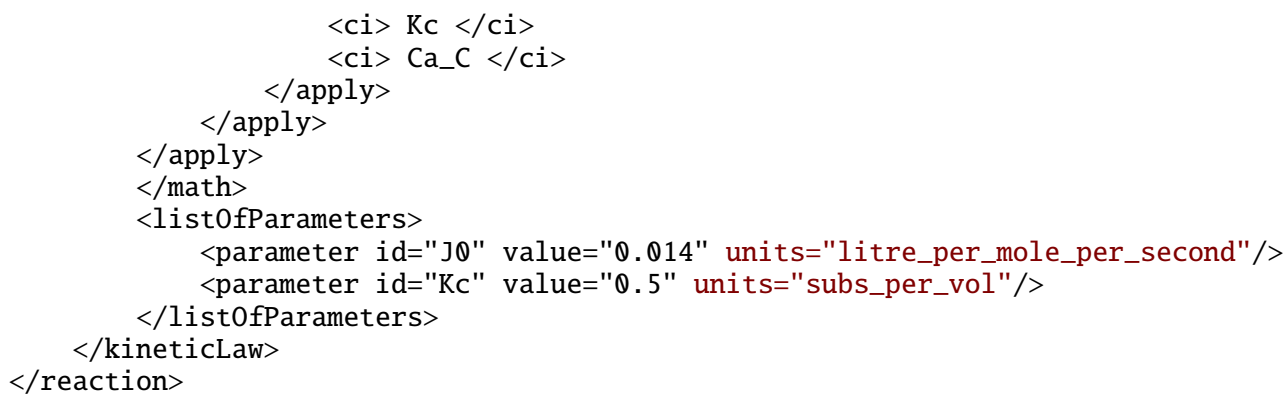




\section{Discussion}

The volume of data now emerging from molecular biotechnology leave little doubt that extensive computerbased modeling, simulation and analysis will be critical to understanding and interpreting the data (Abbott, 1999; Gilman, 2000; Popel and Winslow, 1998; Smaglik, 2000). This has lead to an explosion in the development of computer tools by many research groups across the world. The explosive rate of progress is exciting, but the rapid growth of the field is accompanied by problems and pressing needs.

One problem is that simulation models and results often cannot be directly compared, shared or re-used, because the tools developed by different groups often are not compatible with each other. As the field of systems biology matures, researchers increasingly need to communicate their results as computational models rather than box-and-arrow diagrams. They also need to reuse published and curated models as library components in order to succeed with large-scale efforts (e.g., the Alliance for Cellular Signaling; Gilman, 2000; Smaglik, 2000). These needs require that models implemented in one software package be portable to other software packages, to maximize public understanding and to allow building up libraries of curated computational models.

We offer SBML to the systems biology community as a suggested format for exchanging models between simulation/analysis tools. SBML is an open model representation language oriented specifically towards representing systems of biochemical reactions.

Our vision for SBML is to create an open standard that will enable different software tools to exchange computational models. SBML is not static; we continue to develop and experiment with it, and we interact with other groups who seek to develop similar markup languages. We plan on continuing to evolve SBML with the help of the systems biology community to make SBML increasingly more powerful, flexible and useful.

\subsection{Future enhancements: SBML Level 3 and beyond}

Many people have expressed a desire to see additional capabilities added to SBML. The following summarizes additional features that are under consideration to be included in SBML Level 3; additional information is available in the wiki on the SBML project website (http://sbml.org).

- Arrays. This will enable the creation of arrays of components (species, reactions, compartments and submodels).

- Connections. This will be a mechanism for describing the connections between items in an array.

- Geometry. This will enable the encoding of the spatial characteristics of models including the geometry of compartments, the diffusion properties of species and the specification of different species concentrations across different regions of a cell.

- Model Composition. This will enable a large model to be built up out of instances of other models. It will also allow the reuse of model components and the creation of several instances of the same model.

- Multistate and Complex Species. This will allow the straight-forward construction of models involving species with a large number of states or species composed of subcomponents. The representation scheme would be designed to contain the combinatorial explosion of objects that often results from these types of models.

- Diagrams. This feature will allow components to be annotated with data to enable the display of the model in a diagram.

- Dynamic Structure. This will enable model structure to vary during simulation. One aspect of this allowing rules and reactions to have their effect conditional on the state of the model system. For example in SBML Level 2 it is possible to create a rule with the effect:

$$
\frac{d s}{d t}= \begin{cases}0 & \text { if } s>0 \\ y & \text { otherwise }\end{cases}
$$


Dynamic restructuring would enable the expression of the following example:

$$
\text { if } s>0 \quad \frac{d s}{d t}=y
$$

where $s$ is not determined by the rule when $s \leq 0$.

- Tie-breaking algorithm. This will include a controlled vocabulary and associated attributes on models to indicate the simultaneous event tie-breaking algorithm required to correctly simulate the model.

- Distributions. This will provide a means of specifying random variables and statistical distribution of values. 


\section{Acknowledgments}

This document benefited from repeated reviews and feedback by many people. We give special thanks to James Schaff, Martin Ginkel, Sven Sahle, Ralph Gauges, Ion Moraru, Rainer Machné, Robert Phair, Ben Bornstein, Bruce Shapiro, Maria Schilstra, and Herbert Sauro for critical discussions of many issues, including the implementation of software supporting SBML and the simulation of models.

The development of SBML was originally funded entirely by the Japan Science and Technology Agency (JST) under the ERATO Kitano Symbiotic Systems Project during the years 2000-2003. The principal investigators were Hiroaki Kitano and John C. Doyle. The original SBML Team was lead by Hamid Bolouri and consisted of Hamid Bolouri, Andrew Finney, Herbert Sauro, and Michael Hucka.

We gratefully acknowledge sponsorship from many funding agencies. Support for the continued development of SBML and associated software, meetings and activities comes from the following sources: the National Institute of General Medical Sciences (USA) via grant numbers GM070923 and GM077671; the National Human Genome Research Institute (USA); the International Joint Research Program of NEDO (Japan); the JST ERATO-SORST Program (Japan); the Japanese Ministry of Agriculture; the Japanese Ministry of Education, Culture, Sports, Science and Technology; the BBSRC e-Science Initiative (UK); the DARPA IPTO Bio-Computation Program (USA); and the Air Force Office of Scientific Research (USA). Additional support has been or continues to be provided by the California Institute of Technology (USA), the University of Hertfordshire (UK), the Molecular Sciences Institute (USA), and the Systems Biology Institute (Japan).

SBML was first conceived at the JST/ERATO-sponsored First Workshop on Software Platforms for Systems Biology, held in April, 2000, at the California Institute of Technology in Pasadena, California, USA. The participants collectively decided to begin developing a common XML-based declarative language for representing models. A draft version of the Systems Biology Markup Language was developed by the Caltech ERATO team and delivered to all collaborators in August, 2000. This draft version underwent extensive discussion over mailing lists and then again during the Second Workshop on Software Platforms for Systems Biology held in Tokyo, Japan, November 2000. A revised version of SBML was issued by the Caltech ERATO team in December, 2000, and after further discussions over mailing lists and in meetings, we produced a specification for SBML Level 1 (Hucka et al., 2001).

SBML Level 2 was conceived at the 5th Workshop on Software Platforms for Systems Biology, held in July 2002, at the University of Hertfordshire, UK. The participants collectively decided to revise the form of SBML in SBML Level 2. The first draft of the Level 2 Version 1 document was released in August 2002. The final set of features in SBML Level 2 Version 1 was finalized in May 2003 at the 7 th Workshop on Software Platforms for Systems Biology in Ft. Lauderdale, Florida. SBML Level 2 Version 2 was largely finalized after the 2005 SBML Forum meeting in Boston and a final document was issued in September 2006.

SBML Level 2 Version 3 was finalized after the 2006 SBML Forum meeting in Yokohama, Japan, and the 2007 SBML Hackathon in Newcastle, UK. It was developed with contributions from so many people constituting the worldwide $S B M L$ Forum that we regret it has become infeasible to list individuals by name. We are grateful to everyone on the sbml-discuss@caltech.edu and libsbml-discuss@caltech.edu mailing lists, the creators of CellML (Hedley et al., 2001), the members of the DARPA Bio-SPICE project, and the authors of the following software systems: BALSA, BASIS, BIOCHAM, BioCharon, ByoDyn, BioCyc, BioGrid, BioModels, BioNetGen, BioPathwise, Bio Sketch Pad, BioSens, BioSPICE Dashboard, BioSpreadsheet, BioTapestry, BioUML, BSTLab, CADLIVE, CellDesigner, Cellerator, CellML2SBML, Cellware, CL-SBML, CLEML, COPASI, Cyto-Sim, Cytoscape, DBsolve, Dizzy, E-CELL, ecellJ, ESS, FluxAnalyzer, Fluxor, Gepasi, Gillespie2, HSMB, HybridSBML, INSILICO discovery, JACOBIAN, Jarnac, JDesigner, JigCell, JSim, JWS Online, KEGG2SBML, Kineticon, libSBML, MathSBML, MIRIAM, MesoRD, MetaFluxNet, MMT2, Modesto, Moleculizer, Monod, Narrator, NetBuilder, Oscills, PANTHER Pathway, PathArt, Pathway Analyzer, PathwayLab, PathScout, Pathway Tools, PathwayBuilder, PATIKAweb, PaVESy, PET, PNK, PottersWheel, Reactome, ProcessDB, PROTON, pysbml, PySCeS, RSBML, runSBML, SABIO-RK, SBML ODE Solver, SBML-PET, SBMLeditor, SBMLmerge, SBMLR, SBMLSim, SBMLToolbox, SBliD, SBO, SBToolbox, SBW, SCIpath, Sigmoid, SigPath, SigTran, SIMBA, SimBiology, Simpathica, SimPheny, SimWiz, SloppyCell, SmartCell, SRS Pathway Editor, StochSim, StochKit, STOCKS, TERANODE Suite, Trelis, VANTED, Virtual Cell, WebCell, WinSCAMP, Xholon, and XPPAUT. 


\section{A XML Schema for SBML}

The following is an XML Schema definition for SBML Level 2 Version 3, using the W3C Recommendation for XML Schema version 1.0 of 2 May 2001 (Biron and Malhotra, 2000; Fallside, 2000; Thompson et al., 2000). This Schema does not define all aspects of SBML Level 2: an SBML document validated by this schema is not necessarily a valid SBML Level 2 document. Appendix B contains a a schema for the SBML MathML subset. Appendix C contains a list of the remaining checks required to validate a model in addition to making it consistent with these two schemas.

Note to implementors: the following schema is self-contained and makes reference to the official XML Schema for MathML hosted at the W3. However, for use in software systems, it is more efficient to store the MathML subset Schema of Appendix B in a file on a user's local disk, and change the schemaLocation value on line 27 below to refer to this local copy of the MathML subset Schema. Doing so will avoid requiring a network access every time this SBML Schema is used.

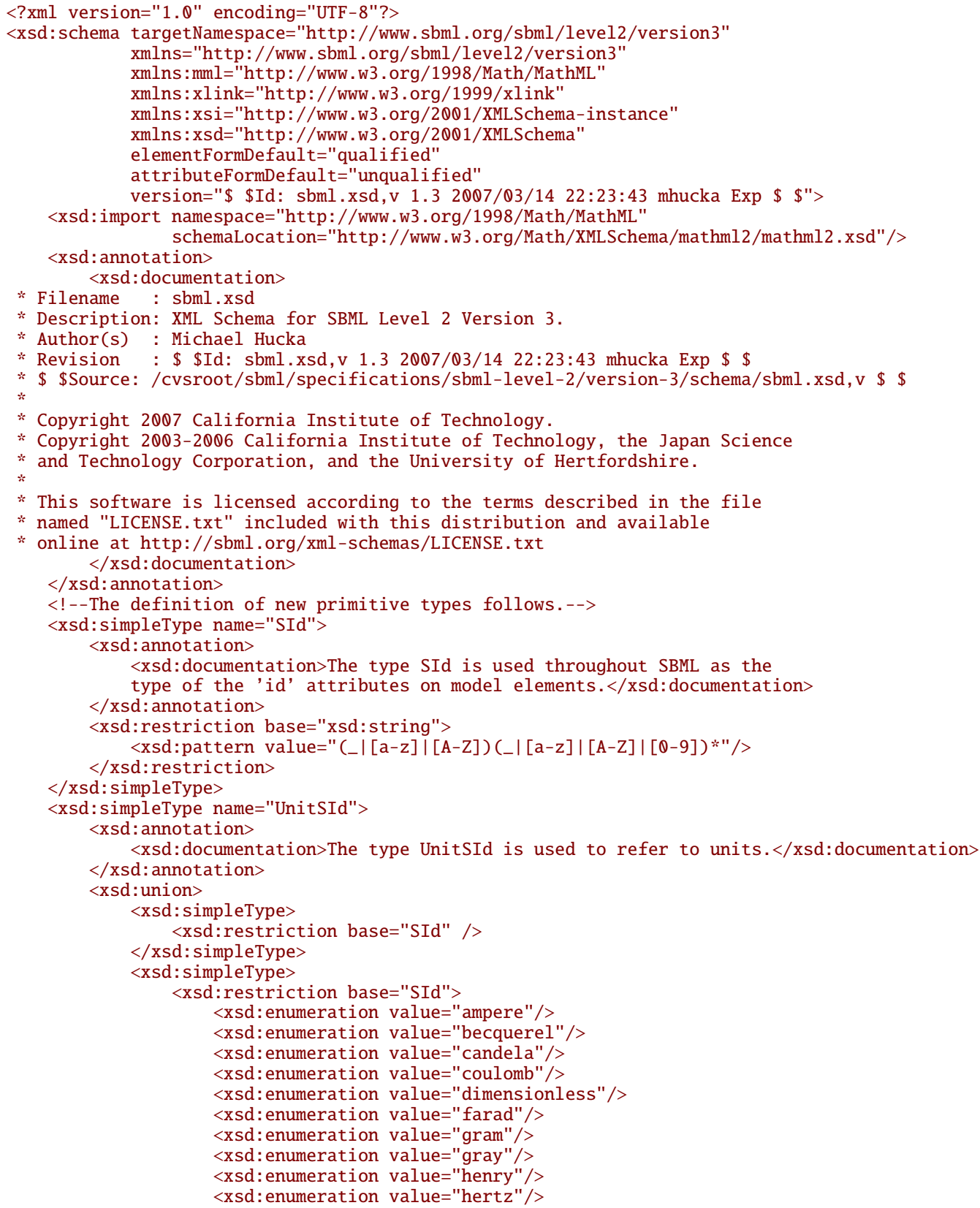


$<$ xsd:enumeration value="item" />

$<$ xsd: enumeration value $="$ joule" $/>$

$<$ xsd:enumeration value="katal"/>

$<$ xsd: enumeration value="kelvin"/>

$<$ xsd: enumeration value="kilogram"/>

$<$ xsd: enumeration value="litre"/>

$<x$ sd:enumeration value="lumen"/>

$<$ xsd: enumeration value $=" l u x " />$

$<$ xsd: enumeration value="metre" $/>$

$<$ xsd: enumeration value $=" m o l e " />$

$<x$ xd: enumeration value="newton" $/>$

$<$ xsd: enumeration value $=" \mathrm{ohm} " />$

$<$ xsd:enumeration value="pascal"/>

$<$ xsd: enumeration value="radian"/>

$<$ xsd: enumeration value $="$ second" $/>$

$<$ xsd: enumeration value="siemens" $/>$

$<$ xsd: enumeration value="sievert"/>

$<$ xsd: enumeration value="steradian"/>

$<$ xsd: enumeration value $="$ tesla" $/>$

$<x$ sd: enumeration value $="$ volt $t " />$

$<x$ sd: enumeration value="watt"/>

$<$ xsd: enumeration value="weber"/>

$</ x s d$ :restriction $>$

$</$ xsd:simpleType $>$

$<$ xsd: simpleType $>$

$<x$ sd:restriction base="SId" $>$

<xsd:enumeration value="substance"/>

$<$ xsd: enumeration value="volume"/>

$<$ xsd: enumeration value $=$ "area" $/>$

$<$ xsd: enumeration value="length"/>

$<$ xsd: enumeration value="time"/>

$</$ xsd:restriction $>$

$</$ xsd:simpleType $>$

$</ x s d$ :union $>$

$</ x$ sd:simpleType $>$

$<$ xsd:simpleType name="SBOTerm" $>$

$<$ xsd:annotation $>$

$<$ xsd:documentation $>$ The data type for sboTerm attribute values. $</ x s d$ :documentation $>$

$</ x s d$ :annotation $>$

<xsd:restriction base="xsd:string">

$<$ xsd:pattern value="(SBO: $)([0-9] 7) " />$

$</ x s d$ :restriction $>$

$</ x s d$ : simpleType $>$

$<$ !--The definition of SBase follows.-->

$<x s d$ :complexType name="SBase" abstract="true">

$<$ xsd:annotation $>$

$<x$ sd:documentation $>$ The SBase type is the base type of all main

components in SBML. It supports attaching metadata, notes and

annotations to components.</xsd:documentation $>$

$</ x s d$ :annotation $>$

$<$ xsd: sequence $>$

$<$ xsd:element name="notes" min0ccurs="Q">

$<$ xsd:complexType $>$

$<$ xsd: sequence $>$

<xsd:any namespace="http://www.w3.org/1999/xhtml"

processContents="skip"

$</ x$ sd: sequence $>$

$</$ xsd: complexType $>$

$</ x s d$ : element $>$

$<$ xsd:element name="annotation" min0ccurs="Q">

$<$ xsd:complexType $>$

$<$ xsd: sequence $>$

$<$ xsd:any processContents="skip"

min0ccurs="0" max0ccurs="unbounded"/>

$</ x$ sd: sequence $>$

$</$ xsd:complexType $>$

$</$ xsd:element $>$

$</ x$ sd: sequence $>$

$<x s$ :attribute name="metaid" type="xsd:ID" use="optional"/>

$<$ xsd:attribute name="sboTerm" type="SBOTerm" use="optional"/> $</$ xsd:complexType $>$

$<$ !--The definition of main SBML classes follows.-->

$<x s d$ :complexType name="FunctionDefinition">

$<$ xsd: complexContent $>$

$<$ xsd:extension base="SBase" $>$

$<x$ sd: sequence $>$

<xsd:element ref="mml:math"/>

$</ x s d$ :sequence $>$

$<$ xsd:attribute name="id" type="SId" use="required"/> 


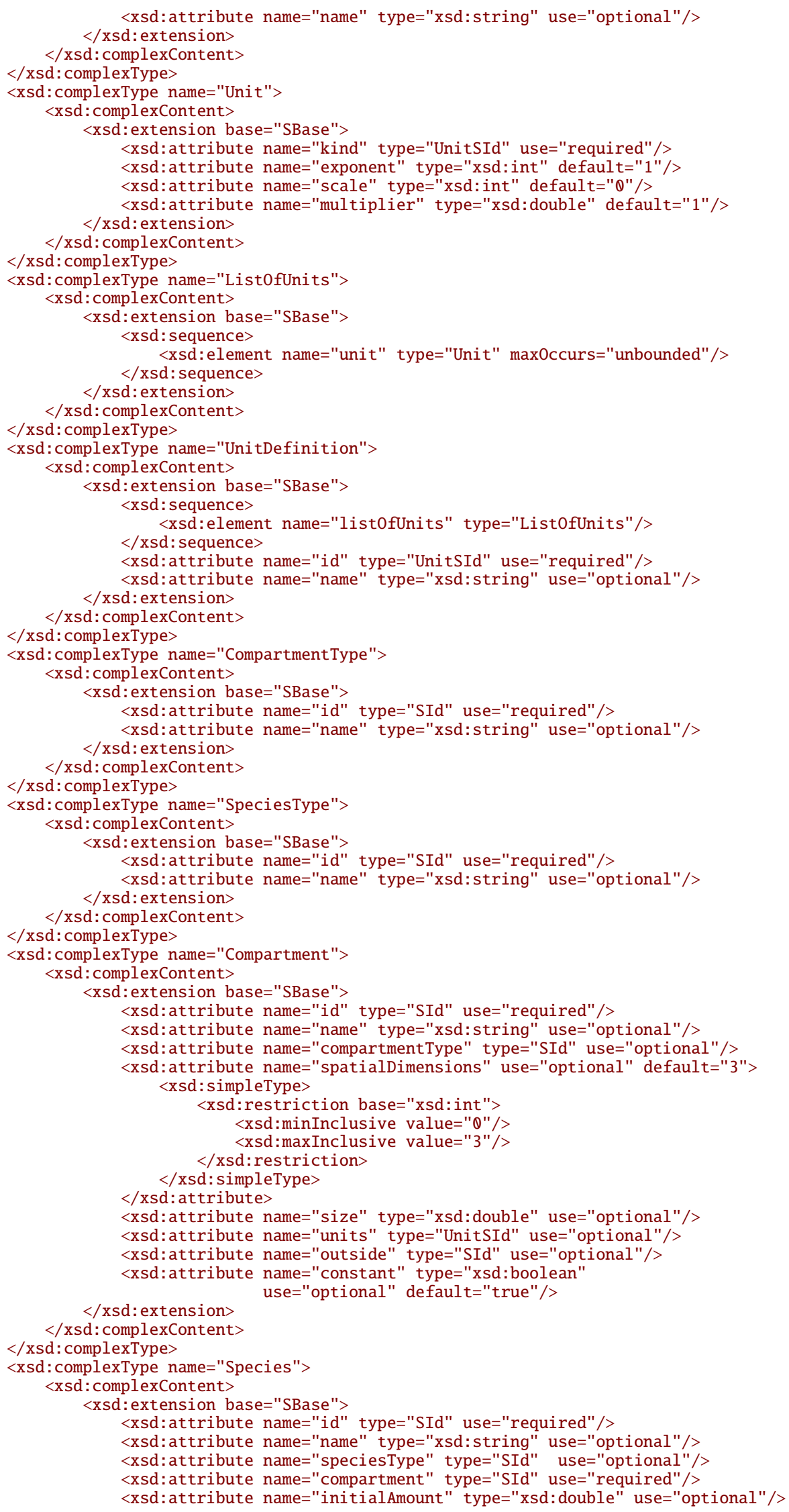


<xsd:attribute name="initialConcentration" type="xsd:double" use="optional"/>

<xsd:attribute name="substanceUnits" type="UnitSId" use="optional"/>

<xsd:attribute name="has0nlySubstanceUnits" type="xsd:boolean" use="optional" default="false"/>

$<$ xsd:attribute name="boundaryCondition" type="xsd:boolean"

use="optional" default="false"/>

<xsd:attribute name="charge" type="xsd:int" use="optional"/>

$<$ xsd:attribute name="constant" type="xsd:boolean"

$</$ xsd:extension $>$ use="optional" default="false"/>

\section{$</ x s d$ : complexContent $>$ \\ $</$ xsd:complexType $>$}

$<$ xsd:complexType name="Parameter" $>$

$<$ xsd: complexContent $>$

$<$ xsd: extension base $=$ "SBase" $>$

$<$ xsd:attribute name="id" type="SId" use="required"/>

$<$ xsd:attribute name="name" type="xsd:string" use="optional"/>

$<$ xsd:attribute name="value" type="xsd:double" use="optional"/>

<xsd:attribute name="units" type="UnitSId" use="optional"/>

$<$ xsd:attribute name="constant" type="xsd:boolean"

$</ x s d$ :extension $>$

use="optional" default="true"/>

$</$ xsd:complexContent $>$

$</$ xsd:complexType $>$

$<$ xsd: complexType name="List0fParameters">

<xsd: complexContent>

$<$ xsd:extension base $="$ SBase" $>$

$<x s d$ : sequence $>$

<xsd:element name="parameter" type="Parameter" max0ccurs="unbounded"/> $</ x$ sd:sequence $>$

$</ x s d$ :extension $>$

$</$ xsd: complexContent $>$

$</$ xsd:complexType $>$

$<$ xsd:complexType name="InitialAssignment">

<xsd: complexContent>

$<$ xsd:extension base="SBase">

$<$ xsd: sequence $>$

<xsd:element ref="mml:math"/>

$</ x s d$ : sequence $>$

$<$ xsd:attribute name="symbol" type="SId" use="required" $/>$

$</$ xsd:extension $>$

$</$ xsd: complexContent $>$

$</$ xsd: complexType $>$

$<$ xsd:complexType name="Rule" abstract="true">

$<$ xsd:complexContent $>$

$<$ xsd: extension base $=$ "SBase" >

$<$ xsd: sequence $>$

<xsd:element ref="mml:math"/>

$</$ xsd: sequence $>$

$</ x s d$ :extension $>$

$</ x s d$ :complexContent $>$

$</$ xsd:complexType $>$

$<$ xsd:complexType name="AlgebraicRule">

$<$ xsd:complexContent>

$<$ xsd: extension base $="$ Rule"/>

$<$ xsd: complexContent $>$

$</$ xsd:complexType $>$

$<$ xsd:complexType name="AssignmentRule">

$<$ xsd: complexContent>

$<$ xsd: extension base="Rule">

$<$ xsd:attribute name="variable" type="SId" use="required"/>

$</ x s d$ : extension $>$

$</$ xsd: complexContent $>$

$</$ xsd:complexType $>$

$<$ xsd:complexType name="RateRule" $>$

$<$ xsd: complexContent>

$<$ xsd:extension base $="$ Rule" $>$

$<x s d$ :attribute name="variable" type="SId" use="required"/>

$</ x s d$ :extension $>$

$</ x s d$ : complexContent $>$

$</$ xsd:complexType $>$

$<$ xsd:complexType name="Constraint">

$<$ xsd:complexContent $>$

$<$ xsd: extension base $=$ "SBase">

$<$ xsd: sequence $>$

<xsd:element ref="mml:math"/>

$<$ xsd:element name="message" minoccurs="Q">

$<$ xsd:complexType $>$

$<$ xsd:sequence $>$

<xsd:any namespace="http://www.w3.org/1999/xhtml" 


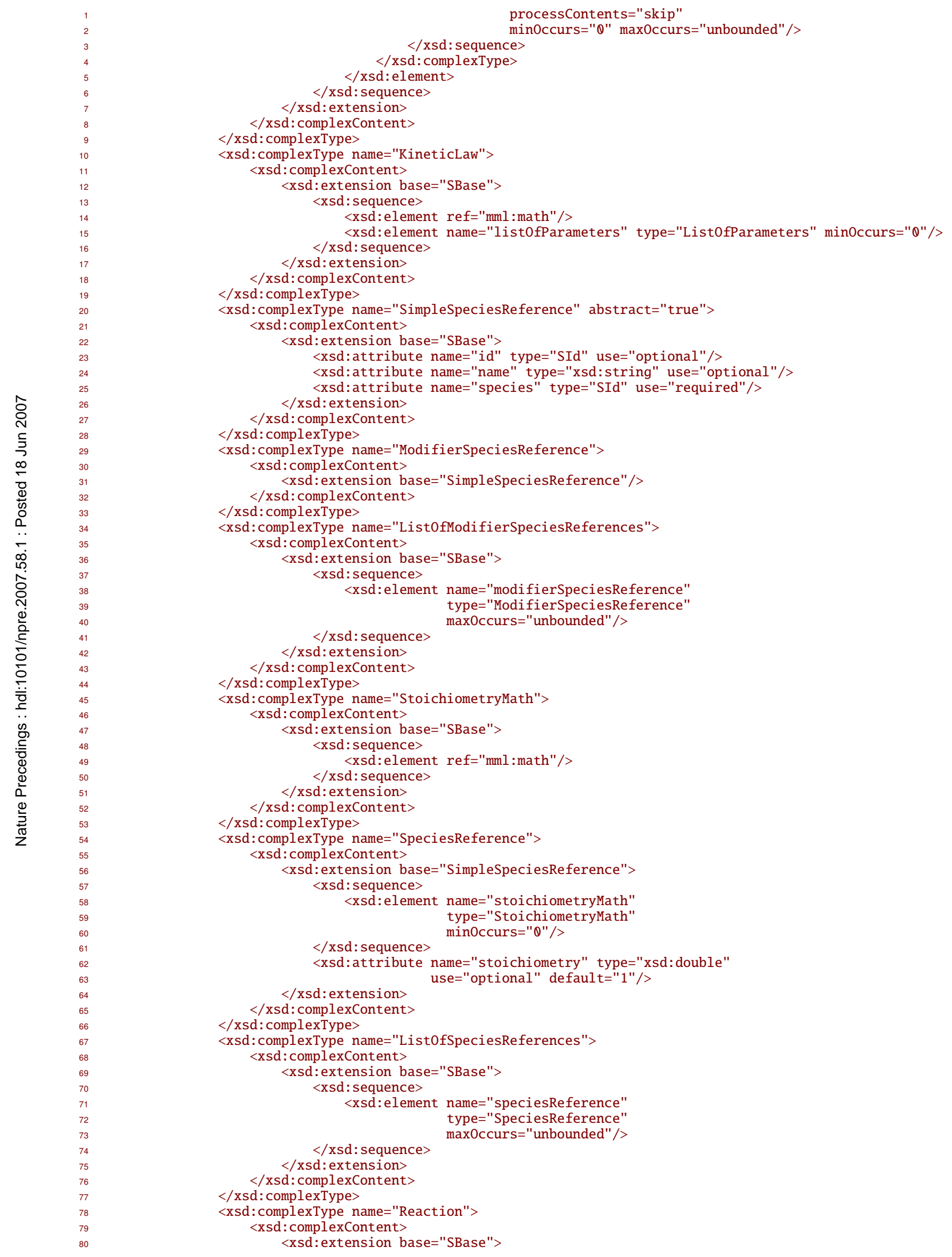


$<$ xsd: sequence $>$

$<$ xsd:element name="list0fReactants" type="ListOfSpeciesReferences" min0ccurs $=" 0 " />$

<xsd:element name="list0fProducts" type="List0fSpeciesReferences" min0ccurs $=" 0 " />$

$<$ xsd:element name="list0fModifiers" type="List0fModifierSpeciesReferences" min0ccurs $=" 0 " />$

$<$ xsd:element name="kineticLaw" type="KineticLaw"

$</$ xsd: sequence $>$ min0ccurs=" $\theta " />$

<xsd:attribute name="id" type="SId" use="required"/>

<xsd:attribute name="name" type="xsd:string" use="optional"/>

$<$ xsd:attribute name="reversible" type="xsd:boolean"

use="optional" default="true"/>
$<x s d: a t t r i b u t e ~ n a m e=" f a s t " ~ t y p e=" x s d: b o o l e a n "$

$</$ xsd:extension $>$

$</$ xsd: complexContent $>$

$<$ xsd:complexType $>$

$<$ xsd:complexType name="EventAssignment" $>$

$<$ xsd: complexContent>

$<$ xsd: extension base $=$ "SBase" $>$

$<$ xsd: sequence $>$

$<$ xsd:element ref="mml:math"/>

$</ x s d$ : sequence $>$

$<$ xsd:attribute name="variable" type="SId" use="required"/>

$</ x s d$ :extension $>$

$</ x s d$ : complexContent $>$

$</$ xsd: complexType $>$

<xsd:complexType name="List0fEventAssignments">

<xsd:complexContent>

$<$ xsd: extension base $=$ "SBase">

$<$ xsd: sequence $>$

<xsd:element name="eventAssignment"

type="EventAssignment"

max0ccurs="unbounded" />

$</ x s d$ : sequence $>$

$</$ xsd:extension $>$

$</$ xsd: complexContent $>$

$</$ xsd:complexType $>$

$<$ xsd:complexType name="Trigger" $>$

$<$ xsd: complexContent $>$

$<$ xsd:extension base $=$ "SBase" $>$

$<$ xsd: sequence $>$

<xsd:element ref="mml:math"/>

$</$ xsd:sequence $>$

$</ x s d$ :extension $>$

$</ x s d$ :complexContent $>$

$</$ xsd:complexType $>$

$<$ xsd:complexType name="Delay">

$<$ xsd: complexContent $>$

$<$ xsd:extension base $=$ "SBase" $>$

$<x s d$ : sequence $>$

$<$ xsd:element ref="mml:math"/>

$</ x s d$ : sequence $>$

$</ x s d$ :extension $>$

$</$ xsd:complexContent $>$

$<$ xsd:complexType $>$

$<$ xsd:complexType name="Event" $>$

$<$ xsd: complexContent>

$<$ xsd:extension base $=$ "SBase" $>$

$<x s d$ : sequence $>$

<xsd:element name="trigger" type="Trigger"/>

$<$ xsd:element name="delay" type="Delay" min0ccurs=" 0 " $/>$

$<$ xsd:element name="list0fEventAssignments" type="ListOfEventAssignments"/>

$</ x$ sd: sequence $>$

<xsd:attribute name="id" type="SId" use="optional"/>

$<$ xsd:attribute name="name" type="xsd:string" use="optional"/>

$</ x s d$ :extension $>$

$</ x s d$ : complexContent $>$

$</$ xsd:complexType $>$

$<$ xsd:complexType name="Model">

$<$ xsd: complexContent>

$<$ xsd: extension base="SBase">

$<$ xsd: sequence $>$

<xsd:element name="list0fFunctionDefinitions" min0ccurs=" $\theta$ ">

$<$ xsd: complexType $>$

<xsd:complexContent>

$<$ xsd: extension base="SBase">

$<\mathrm{xsd}$ : sequence $>$ 


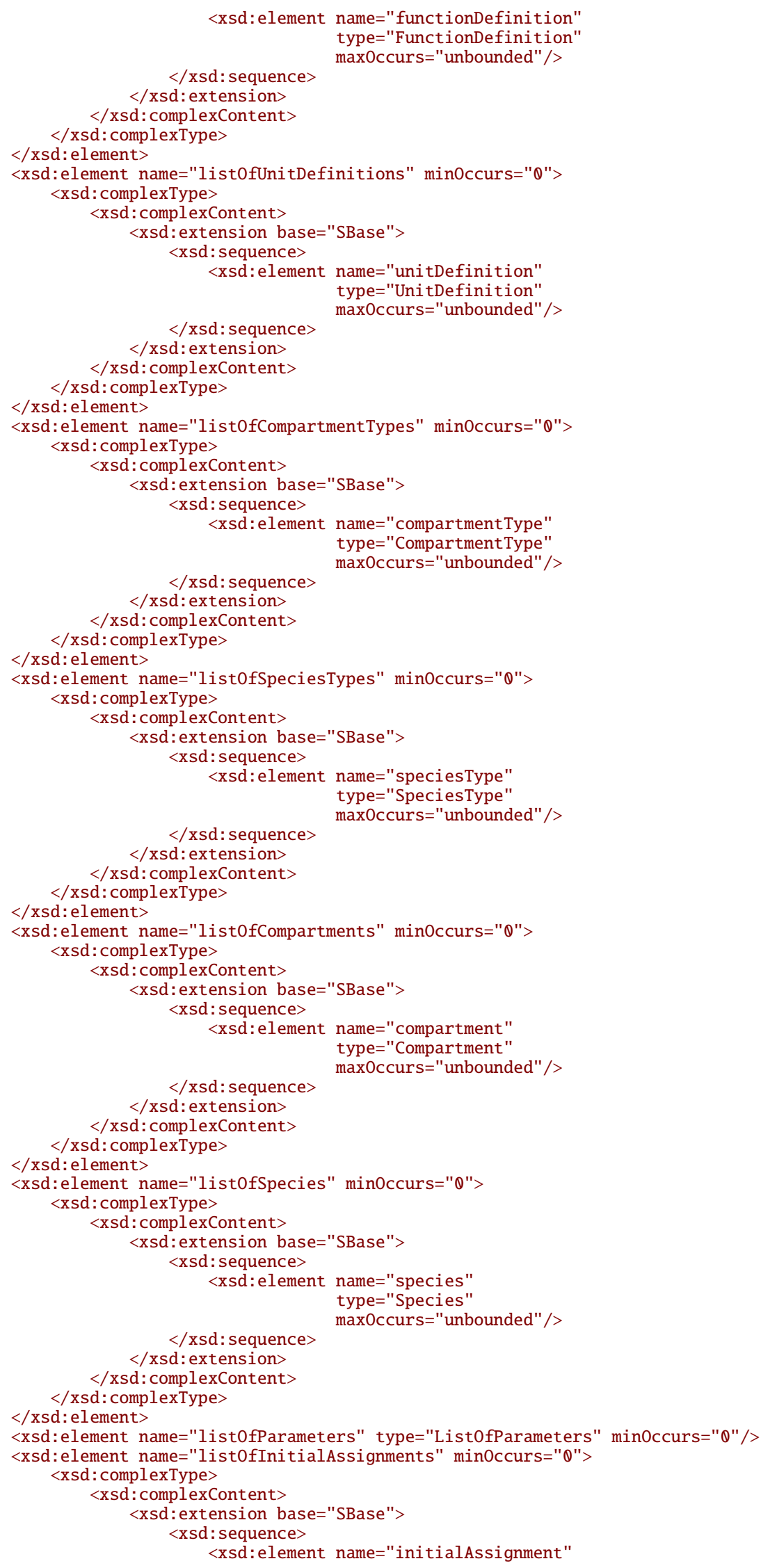


type="InitialAssignment"

max0ccurs="unbounded" />

$</ x s d$ :sequence $>$

$</$ xsd:extension $>$

$</$ xsd: complexContent $>$

$</ x s d$ :complexType $>$

$</ x s d:$ element $>$

$<$ xsd:element name="list0fRules" min0ccurs=" $\theta ">$

$<$ xsd: complexType >

$<$ xsd:complexContent $>$

<xsd: extension base="SBase">

$<$ xsd:choice max0ccurs="unbounded">

<xsd:element name="algebraicRule"

type="AlgebraicRule"

min0ccurs $=" \theta " />$

$<$ xsd:element name="assignmentRule"

type="AssignmentRule"

minoccurs $=" \theta " />$

$<$ xsd:element name="rateRule"

type $=$ "RateRule"

$</ x s d$ :choice $>$

min0ccurs $=" \theta " />$

$</ x s d$ :extension $>$

$</ x s d$ :complexContent $>$

$</ x s d$ : complexType $>$

$</$ xsd:element $>$

$<$ xsd:element name="list0fConstraints" min0ccurs $=" \otimes ">$

$<x s d$ : complexType $>$

$<$ xsd:complexContent $>$

$<$ xsd: extension base $=$ "SBase" $>$

$<x s d$ : sequence $>$

<xsd:element name="constraint"

type="Constraint

max0ccurs="unbounded"/>

$</ x s d$ : sequence $>$

$</$ xsd:extension $>$

$<$ /xsd:complexContent $>$

$</ x s d$ : complexType $>$

$</$ xsd:element $>$

$<$ xsd:element name="list0fReactions" min0ccurs $=" 0 ">$

$<$ xsd:complexType $>$

$<$ xsd: complexContent $>$

$<$ xsd:extension base $=$ "SBase" $>$

$<$ xsd: sequence $>$

$<$ xsd:element name="reaction"

type $=$ "Reaction"

max0ccurs="unbounded"/>

$</ x$ sd: sequence $>$

$</ x s d$ : extension $>$

$</ x s d$ :complexContent $>$

$</$ xsd:complexType $>$

$</ x s d:$ element $>$

<xsd:element name="list0fEvents" min0ccurs=" $0 ">$

$<$ xsd:complexType >

$<$ xsd:complexContent $>$

$<$ xsd: extension base="SBase">

$<x s d$ : sequence $>$

$<x s d: e l e m e n t$ name="event"

type="Event"

$</$ xsd:sequence $>$

max0ccurs="unbounded"/>

$</ x s d$ :extension $>$

$</ x s d$ : complexContent $>$

$</$ xsd:complexType $>$

$</$ xsd:element $>$

$</ x s d$ : sequence $>$

<xsd:attribute name="id" type="SId" use="optional"/>

$<$ xsd:attribute name="name" type="xsd:string" use="optional"/>

$</ x s d$ : extension $>$

$</$ xsd: complexContent $>$

$</$ xsd: complexType $>$

$<$ !-- The following is the type definition for the top-level element in an SBML document.-->

$<$ xsd: complexType name="Sbml" $>$

$<$ xsd:complexContent >

$<$ xsd:extension base="SBase">

$<x s d$ :sequence $>$

<xsd:element name="model" type="Model"/>

$</ x s d$ : sequence $>$

$<$ xsd:attribute name="level" type="xsd:positiveInteger"

use="required" fixed="2"/> 


\author{
$<x s d: a t t r i b u t e$ name="version" type="xsd:positiveInteger" \\ $</$ xsd:extension $>$ \\ $</$ xsd:complexContent $>$ \\ $</$ xsd:complexType $>$ \\ $<$ !--The following is the (only) top-level element allowed in an SBML document.--> \\ $<x s d$ :element name="sbml" type="Sbml"/> \\ $<$ !--The end. - - > \\ $</$ xsd:schema $>$
}

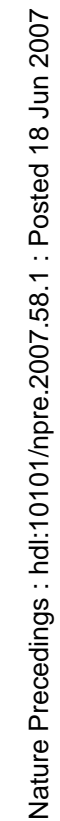




\section{B XML Schema for MathML subset}

The following XML schema defines the syntax of the MathML syntax that is used in SBML Level 2.

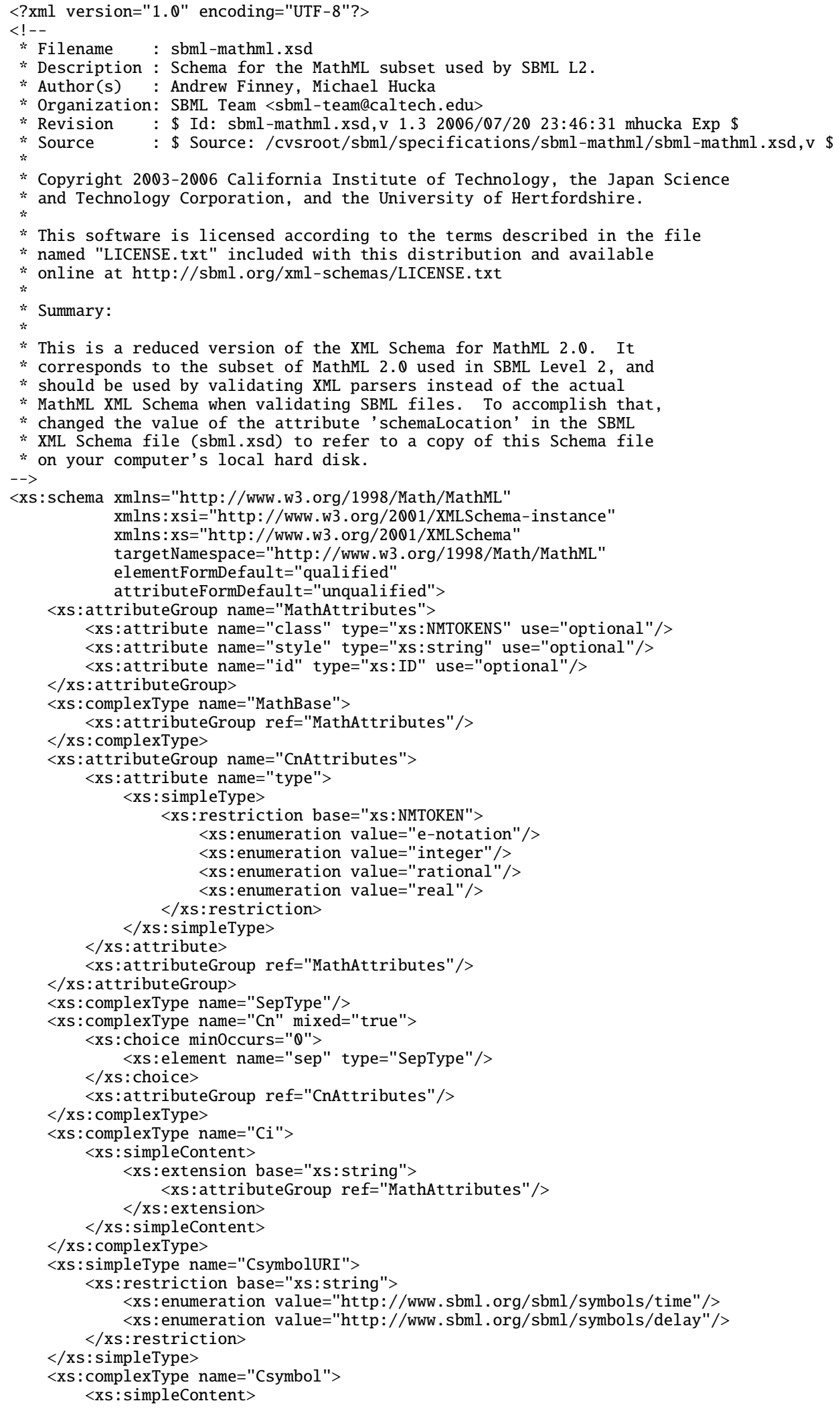


$<$ xs: extension base="xs: string">

$<$ xs:attribute name="encoding" use="required" fixed="text"/>

$<x s$ :attribute name="definitionURL" type="CsymbolURI" use="required"/>

$<$ xs:attributeGroup ref="MathAttributes"/>

$</ x$ : extension $>$

$</ x s$ : simpleContent $>$

$</ x s$ : complexType $>$

$<$ xs: complexType name="NodeContainer">

<xs: complexContent>

$<$ xs: extension base $=$ "MathBase" $>$

$<$ xs: group ref="Node"/>

$</ x s$ : extension $>$

$</$ xs: complexContent $>$

$</ x s$ : complexType $>$

$<x$ : complexType name="Apply" $>$

$<$ xs: complexContent $>$

$<$ xs: extension base="MathBase" $>$

$<x s$ : sequence $>$

$<$ xs: choice $>$

$<$ xs:element name="ci" type="Ci"/>

$<x$ :element name="csymbol" type="Csymbol"/>

$<x s$ :element name="eq" type="MathBase"/>

$<$ xs:element name="neq" type $=$ "MathBase" $/>$

$<$ xs:element name="gt" type="MathBase"/>

$<x$ : element name="lt" type="MathBase"/>

$<x$ : element name="geq" type="MathBase" $/>$

$<$ xs:element name="leq" type="MathBase" $/>$

$<$ xs:element name="plus" type="MathBase" $/>$

$<$ xs:element name="minus" type $=$ "MathBase" $/>$

$<x$ :element name="times" type $=$ "MathBase"/>

$<$ xs:element name="divide" type="MathBase" $/>$

$<$ xs:element name="power" type="MathBase"/>

$<x$ : sequence $>$

$<$ xs:element name="root" type="MathBase"/>

$<x s:$ element name="degree" type="NodeContainer" min0ccurs=" $0 " />$

$</ x s$ : sequence $>$

$<$ xs:element name="abs" type="MathBase" $/>$

$<$ xs:element name="exp" type="MathBase" $/>$

$<$ xs:element name="ln" type="MathBase"/>

$<$ xs: sequence $>$

$<$ xs:element name="log" type="MathBase"/>

$<x s$ :element name="logbase" type="NodeContainer" min0ccurs="Q"/>

$</ x s$ : sequence $>$

$<$ xs:element name="floor" type="MathBase" $/>$

$<$ xs:element name="ceiling" type $=$ "MathBase" $/>$

$<x s$ :element name="factorial" type="MathBase" $/>$

$<x$ : element name="and" type="MathBase"/>

$<$ xs:element name="or" type="MathBase"/>

$<$ xs:element name $=$ "xor" type $=$ "MathBase" $/>$

$<x$ : element name="not" type $=$ "MathBase" $/>$

$<$ xs:element name="sin" type="MathBase" $/>$

$<$ xs:element name $=$ "cos" type $=$ "MathBase" $/>$

$<x s$ :element name $="$ tan" type $="$ MathBase" $/>$

$<x s:$ element name="sec" type="MathBase" $/>$

$<$ xs:element name="csc" type="MathBase" $/>$

$<x$ : element name="cot" type="MathBase" $/>$

$<x$ : element name="sinh" type="MathBase"/>

$<$ xs:element name="cosh" type="MathBase"/>

$<x$ : element name="tanh" type="MathBase"/>

$<x s$ :element name="sech" type="MathBase"/>

$<x$ :element name="csch" type="MathBase" $/>$

$<x$ : element name="coth" type="MathBase"/>

$<x$ : element name="arcsin" type $=$ "MathBase" />

$<$ xs:element name="arccos" type $=$ "MathBase" $/>$

$<$ xs:element name $=$ "arctan" type $=$ "MathBase" $/>$

$<x$ : element name="arcsec" type="MathBase" $/>$

$<x s$ :element name="arccsc" type="MathBase" $/>$

$<x s$ :element name="arccot" type="MathBase"/>

$<x$ : element name="arcsinh" type="MathBase"/>

$<x$ : element name="arccosh" type="MathBase"/>

$<x$ : element name="arctanh" type="MathBase" $/>$

$<x s:$ element name="arcsech" type $=$ "MathBase"/>

$<x$ : element name="arccsch" type="MathBase"/>

$<x$ :element name="arccoth" type="MathBase"/>

$</ x s$ : choice $>$

$<$ xs:group ref="Node" max0ccurs="unbounded"/>

$</ x$ : : sequence $>$

$</ x s$ : extension $>$

$</$ xs: complexContent $>$

$</$ xs: complexType $>$ 


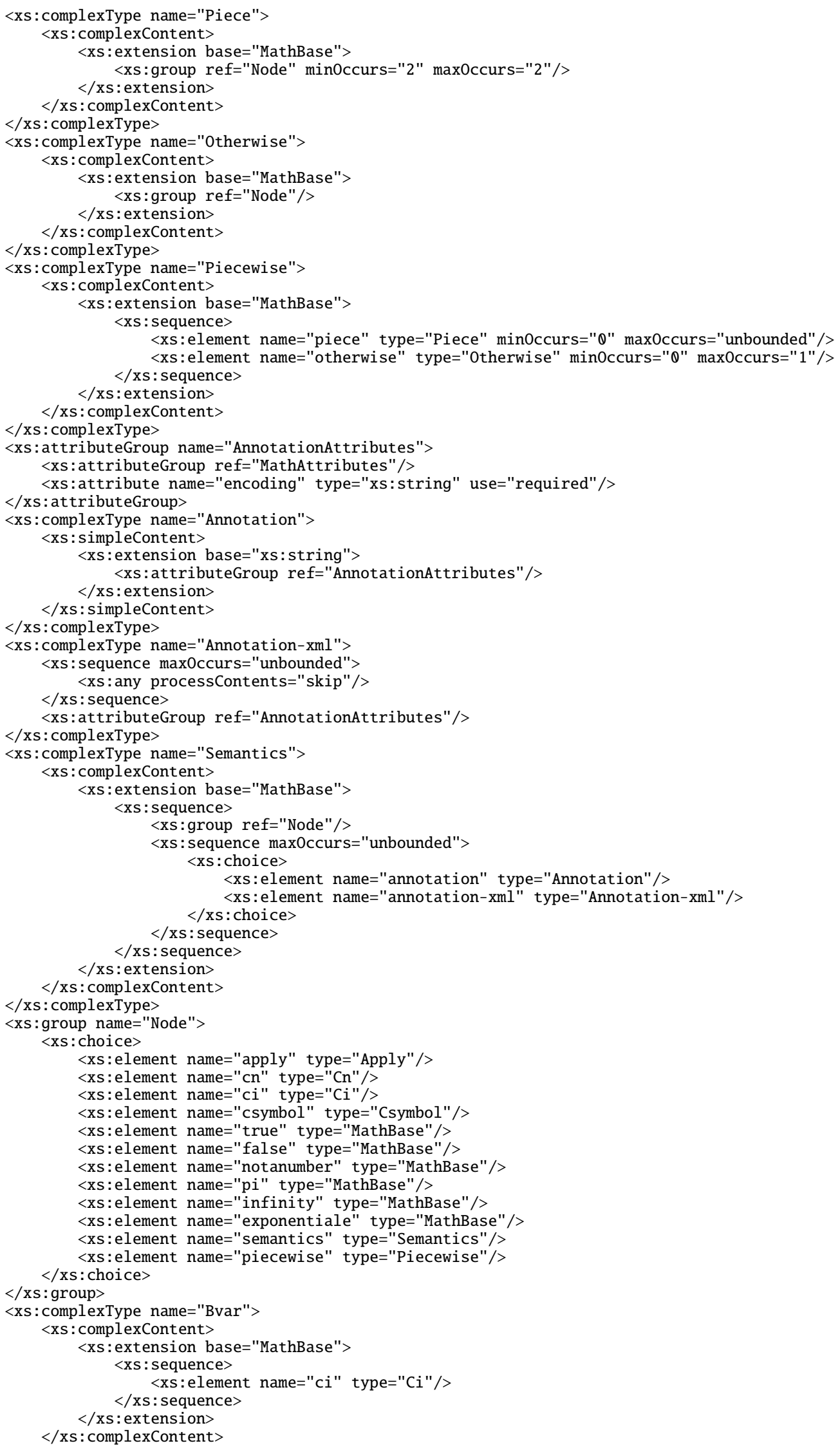


$</$ xs: complexType $>$

$<$ xs: complexType name="Lambda" $>$

$<$ xs: complexContent>

$<$ xs: extension base $="$ MathBase"> $<x s$ : sequence $>$

<xs:element name="bvar" type="Bvar" min0ccurs="0" max0ccurs="unbounded"/> $<$ xs:group ref="Node"/> $</ x s$ : sequence $>$

$</$ xs: extension $>$

$</ x s$ : complexContent $>$

$</ x s$ : complexType $>$

$<$ xs: complexType name="Math">

$<$ xs: complexContent $>$

$<$ xs: extension base $=$ "MathBase" $>$

$<$ xs: choice $>$

$<$ xs: group ref="Node"/>

$<$ xs:element name="lambda" type="Lambda"/>

$</ x$ : choice $>$

$</ x$ s:extension $>$

$</ x s$ : complexContent $>$

$</ x s$ : complexType $>$

$<$ xs:element name="math" type="Math">

$<$ !--This is the top-level element for a 'math' container in SBML.-->

$</ x s$ :element $>$

$</ x s$ : schema $>$ 


\section{Validation rules for SBML}

This section contains a summary of all the conditions that should be true of a model, in addition to consistency with the XML Schemas given in Appendixes A and B, for that model to considered valid SBML.

\section{General XML validation}

10101. An SBML XML file must use UTF-8 as the character encoding. More precisely, the encoding attribute of the XML declaration at the beginning of the XML data stream cannot have a value other than "UTF-8". An example valid declaration is <?xml version="1.0" encoding="UTF-8"?>. (References: L2V2 Section 4.1; L2V3 Section 4.1.)

10102. An SBML XML document must not contain undefined elements or attributes in the SBML namespace. Documents containing unknown elements or attributes placed in the SBML namespace do not conform to the SBML Level 2 specification. (References: L2V2 Section 4.1; L2V3 Section 4.1.)

10103. An SBML XML document must conform to the XML Schema for the corresponding SBML Level, Version and Release. The XML Schema for SBML defines the basic SBML object structure, the data types used by those objects, and the order in which the objects may appear in an SBML document. (References: SBML L2V2 Section 4.1; L2V3 Section 4.1.)

\section{General MathML validation}

10201. All MathML content in SBML must appear within a math element, and the math element must be either explicitly or implicitly in the XML namespace "http://www.w3.org/1998/Math/MathML". (References: L2V2 Section 3.5; L2V3 Section 3.4.)

10202. The only permitted MathML 2.0 elements in SBML Level 2 are the following: cn, ci, csymbol, sep, apply, piecewise, piece, otherwise, eq, neq, gt, lt, geq, leq, plus, minus, times, divide, power, root, abs, exp, ln, log, floor, ceiling, factorial, and, or, xor, not, degree, bvar, logbase, sin, cos, tan, sec, csc, cot, sinh, cosh, tanh, sech, csch, coth, arcsin, arccos, arctan, arcsec, arccsc, arccot, arcsinh, arccosh, arctanh, arcsech, arccsch, arccoth, true, false, notanumber, pi, infinity, exponentiale, semantics, annotation, and annotation-xml. (References: L2V2 Section 3.5.1; L2V3 Section 3.4.1.)

10203. In the SBML subset of MathML 2.0, the MathML attribute encoding is only permitted on csymbol. No other MathML elements may have the encoding attribute. (References: L2V2 Section 3.5.1; L2V3 Section 3.4.1.)

10204. In the SBML subset of MathML 2.0, the MathML attribute definitionURL is only permitted on csymbol. No other MathML elements may have a definitionURL attribute. (References: L2V2 Section 3.5.1; L2V3 Section 3.4.1.)

10205. In SBML Level 2, the only values permitted for the definitionURL attribute on a csymbol element are "http://www.sbml.org/sbml/symbols/time" and "http://www.sbml.org/sbml/symbols/delay". (References: L2V2 Section 3.5.5; L2V3 Section 3.4.6.)

10206. In the SBML subset of MathML 2.0, the MathML attribute type is only permitted on the cn construct. No other MathML elements may have a type attribute. (References: L2V2 Section 3.5.1; L2V3 Section 3.4.1.)

10207. The only permitted values for the type attribute on MathML cn elements are "e-notation", "real", "integer", and "rational". (References: L2V2 Section 3.5.2; L2V3 Section 3.4.2.)

10208. MathML lambda elements are only permitted as the first element inside the math element of a FunctionDefinition or as the first element of a semantics element immediately inside the math element of a FunctionDefinition; they may not be used elsewhere in an SBML model. (References: L2V2 Sections 3.5.1 and 4.3; L2V3 Sections 3.4.1 and 4.3.2.) 
10209. The arguments of the MathML logical operators and, or, xor, and not must have boolean values. (References: L2V2 Section 3.5.8; L2V3 Section 3.4.9.)

10210. The arguments to the following MathML constructs must evaluate to be numbers (i.e., MathML, real, integer, rational, or "e-notation" numbers, or the time or delay csymbol): plus, minus, times, divide, power, root, abs, exp, ln, log, floor, ceiling, factorial, sin, cos, tan, sec, csc, cot, sinh, cosh, tanh, sech, csch, coth, arcsin, arccos, arctan, arcsec, arccsc, arccot, arcsinh, arccosh, arctanh, arcsech, arccsch, arccoth. (References: L2V2 Section 3.5.8; L2V3 Section 3.4.9.)

10211. The values of all arguments to eq and neq operators should have the same type (either all boolean or all numerical). (References: L2V2 Section 3.5.8; L2V3 Section 3.4.9.)

10212. The types of values within piecewise operators should all be consistent: the set of expressions that make up the first arguments of the piece and otherwise operators within the same piecewise operator should all return values of the same type. (References: L2V2 Section 3.5.8; L2V3 Section 3.4.9.)

10213. The second argument of a MathML piece operator must have a boolean value. (References: L2V2 Section 3.5.8; L2V3 Section 3.4.9.)

10214. Outside of a FunctionDefinition, if a ci element is the first element within a MathML apply, then the ci's value can only be chosen from the set of identifiers of FunctionDefinition instances defined in the enclosing SBML Model instance. (References: L2V2 Section 4.3.2; L2V3 Section 4.3.2.)

10215. Outside of a FunctionDefinition, if a ci element is not the first element within a MathML apply, then the ci's value can only be chosen from the set of identifiers of Species, Compartment, Parameter or Reaction objects defined in the SBML model. (References: L2V2 Section 4.3.2; L2V3 Section 3.4.3.)

10216. The id value of a Parameter defined within a KineticLaw can only be used in ci elements within the MathML content of that same KineticLaw; the identifier is not visible to other parts of the model. (References: L2V2 Sections 3.4.1, 3.5.3 and 4.13.5; L2V3 Sections 3.3.1, 3.4.3 and 4.13.5.)

10217. The MathML formulas in the following elements must yield numerical values (that is, MathML real, integer or "e-notation" numbers, or the time or delay csymbol): math in KineticLaw, stoichiometryMath in SpeciesReference, math in InitialAssignment, math in AssignmentRule, math in RateRule, math in AlgebraicRule, math in Event Delay, and math in EventAssignment. (References: L2V2 Section 4.10, 4.11, 4.13 and 4.14; L2V3 Sections 4.10, 4.11, 4.13 and 4.14.)

10218. A MathML operator must be supplied the number of arguments appropriate for that operator. (References: L2V2 Section 3.5.1; L2V3 Section 3.4.1.)

\section{General identifier validation}

10301. The value of the id attribute on every instance of the following classes of objects in a model must be unique across the set of all id values in a model: Model, FunctionDefinition, CompartmentType, SpeciesType, Compartment, Species, Reaction, SpeciesReference, ModifierSpeciesReference, Event, and model-wide Parameters. Exception: the identifiers of instances of UnitDefinition, and parameters defined inside a reaction, are treated separately. (References: L2V1 Section 3.5; L2V2 Section 3.4.1; L2V3 Section 3.3)

10302. The value of the id attribute of every UnitDefinition must be unique across the set of all UnitDefinitions in the entire model. (References: L2V1 Section 3.5 and 4.4; L2V2 Sections 3.4 .1 and 4.4; L2V3 Sections 3.3 and 4.4.)

10303. The value of the id attribute of each parameter defined locally within a KineticLaw must be unique across the set of all such parameter definitions in that KineticLaw. (References: L2V1 Sections 3.4.1 and 4.13.9; L2V2 Sections 3.4.1 and 4.13.5; L2V3 Sections 3.3.1 and 4.13.5.) 
10304. The value of the variable attribute in all AssignmentRule and RateRule definitions must be unique across the set of all such rule definitions in a model. (References: L2V1 Section 4.8.4; L2V2 Section 4.11; L2V3 Section 4.11.3.)

10305. In each Event, the value of the variable attribute within every EventAssignment definition must be unique across the set of all EventAssignments within that Event. In other words, a single Event cannot make more than one assignment to a given identifier. (References: L2V1 erratum 17; L2V2 Section 4.14.2; L2V3 Section 4.14.4.)

10306. An identifier used as the value of variable in an EventAssignment cannot also appear as the value of variable in an AssignmentRule. (References: L2V1 Section 4.10.5; L2V2 Section 4.14.2; L2V3 Section 4.14.4.)

10307. Every metaid attribute value must be unique across the set of all metaid values in a model. (References: L2V2 Sections 3.1.6 and 3.3.1; L2V3 Sections 3.2.1 and 3.1.6.)

10308. The value of a sboTerm attribute must have the data type SBOTerm, which is a string consisting of the characters 'S', 'B', 'O', ':', followed by exactly seven digits. (References: L2V2 Section 3.1.9; L2V3 Section 3.1.9.)

10309. The syntax of metaid attribute values must conform to the syntax of the XML type ID. (References: L2V2 Sections 3.1.6 and 3.3.1; L2V3 Sections 3.2.1 and 3.1.6.)

10310. The syntax of id attribute values must conform to the syntax of the SBML type SId. (References: L2V2 Section 3.1.7; L2V3 Section 3.1.7.)

10311. The syntax of unit identifiers (i.e., the values of the id attribute on UnitDefinition, the units attribute on Compartment, the units attribute on Parameter, and the substanceUnits attribute on Species) must conform to the syntax of the SBML type UnitSId. (References: L2V3 Section 3.1.8.)

\section{General annotation validation}

10401. Every top-level element within an annotation element must have a namespace declared. (References: L2V2 Section 3.3.3; L2V3 Section 3.2.4.)

10402. There cannot be more than one top-level element using a given namespace inside a given annotation element. (References: L2V2 Section 3.3.3; L2V3 Section 3.2.4.)

10403. Top-level elements within an annotation element cannot use any SBML namespace, whether explicitly (by declaring the namespace to be one of the URIs "http://www.sbml.org/sbml/level1", "http://www.sbml.org/sbml/level2", "http://www.sbml.org/sbml/level2/version2", or "http://www.sbml.org/sbml/level2/version3"), or implicitly (by failing to declare any namespace). (References: L2V2 Section 3.3.3; L2V3 Section 3.2.4.)

\section{General unit validation}

10501. The units of the expressions used as arguments to a function call must match the units expected for the arguments of that function. (References: L2V2 Section 3.5; L2V3 Section 3.4.)

10511. When the variable in an AssignmentRule refers to a Compartment, the units of the rule's right-hand side must be consistent with the units of that compartment's size. (References: L2V2 Section 4.11.3; L2V3 Section 4.11.3.)

10512. When the variable in an AssignmentRule refers to a Species, the units of the rule's right-hand side must be consistent with the units of the species' quantity. (References: L2V2 Section 4.11.3; L2V3 Section 4.11.3.) 
10513. When the variable in an AssignmentRule refers to a Parameter, the units of the rule's right-hand side must be consistent with the units declared for that parameter. (References: L2V2 Section 4.11.3; L2V3 Section 4.11.3.)

10521. When the variable in an InitialAssignment refers to a Compartment, the units of the InitialAssignment's math expression must be consistent with the units of that compartment's size. (References: L2V2 Section 4.10.4; L2V3 Section 4.10.)

10522. When the variable in an InitialAssignment refers to a Species, the units of the InitialAssignment's math expression must be consistent with the units of that species' quantity. (References: L2V2 Section 4.10.4; L2V3 Section 4.11.3.)

10523. When the variable in an InitialAssignment refers to a Parameter, the units of the InitialAssignment's math expression must be consistent with the units declared for that parameter. (References: L2V2 Section 4.10.4; L2V3 Section 4.11.3.)

10531. When the variable in a RateRule definition refers to a Compartment, the units of the rule's righthand side must be of the form $x$ per time, where $x$ is either the units in that Compartment definition, or (in the absence of explicit units declared for the compartment size) the default units for that compartment, and time refers to the units of time for the model. (References: L2V2 Section 4.11.4; L2V3 Section 4.11.4.)

10532. When the variable in a RateRule definition refers to a Species, the units of the rule's right-hand side must be of the form $x$ per time, where $x$ is the units of that species' quantity, and time refers to the units of time for the model. (References: L2V2 Section 4.11.4; L2V3 Section 4.11.4.)

10533. When the variable in a RateRule definition refers to a Parameter, the units of the rule's right-hand side must be of the form $x$ per time, where $x$ is the units in that Parameter definition, and time refers to the units of time for the model. (References: L2V2 Section 4.11.4; L2V3 Section 4.11.4.)

10541. The units of the math formula in a KineticLaw definition must be the equivalent of substance per time. (References: L2V2 Section 4.13.5; L2V3 Section 4.13.5.)

10551. The units of the mathematical formula in the delay of an Event must correspond to the model's overall units of time. (L2V3 Section 4.14.3.)

10561. When the variable attribute of an EventAssignment contains the identifier of a Compartment in the model, the units of the mathematical expression in the EventAssignment's math expression must be consistent with the units of that compartment's size. (References: L2V2 Section 4.14.2; L2V3 Section 4.14.4.)

10562. When the variable attribute of an EventAssignment contains the identifier of a Species in the model, the units of the mathematical expression in the EventAssignment's math expression must be consistent with the units of that species' quantity. (References: L2V2 Section 4.14.2; L2V3 Section 4.14.4.)

10563. When the variable attribute of an EventAssignment contains the identifier of a Parameter in the model, the units of the mathematical expression in the EventAssignment's math expression must be consistent with the units declared for that parameter. (References: L2V2 Section 4.14.2; L2V3 Section 4.14.4.)

\section{General model validation}

10601. A system of equations created from an SBML model must not be overdetermined. (References: L2V2 Section 4.11.5; L2V3 Section 4.11.5.) 


\section{General SBO validation}

10701. The value of the sboTerm attribute on a Model must be an SBO identifier referring to a modeling framework defined in SBO. That is, the value must be a term derived from SB0:0000004, "modeling framework". (References: L2V2 Section 4.2.1; L2V3 Section 4.2.2.)

10702. The value of the sboTerm attribute on a FunctionDefinition must be an SBO identifier referring to a mathematical expression. That is, the value must be a term derived from SB0:0000064, "mathematical expression". (References: L2V2 Section 4.3.3; L2V3 Section 4.3.3.)

10703. The value of the sboTerm attribute on a Parameter must be an SBO identifier referring to a quantitative parameter. That is, the value must be a term derived from SB0:0000002, "quantitative parameter". (References: L2V2 Section 4.9.5; L2V3 Section 4.9.5.)

10704. The value of the sboTerm attribute on an InitialAssignment must be an SBO identifier referring to a mathematical expression. That is, the value must be a term derived from SB0:0000064, "mathematical expression". (References: L2V2 Section 4.10.3; L2V3 Section 4.10.3.)

10705. The value of the sboTerm attribute on a AlgebraicRule, RateRule or AssignmentRule must be an SBO identifier referring to a mathematical expression. That is, the value must be a term derived from SB0:0000064, "mathematical expression". (References: L2V2 Section 4.11.1; L2V3 Section 4.11.1.)

10706. The value of the sboTerm attribute on a Constraint must be an SBO identifier referring to a mathematical expression. That is, the value must be a term derived from SB0:0000064, "mathematical expression". (References: L2V2 Section 4.12.3; L2V3 Section 4.12.3.)

10707. The value of the sboTerm attribute on a Reaction must be an SBO identifier referring to a modeling framework. That is, the value must be a term derived from SB0:0000231, "event". (References: L2V2 Section 4.13.1; L2V3 Section 4.13.1.)

10708. The value of the sboTerm attribute on a SpeciesReference or a ModifierSpeciesReference must be an SBO identifier referring to a participant functional role. That is, the value must be a term derived from SB0:0000003, "participant functional type". The appropriate term depends on whether the entity is a reactant, product or modifier. If a reactant, the term should be chosen from the SB0:0000010, "reactant" hierarchy; if a product, the term should be chosen from the SB0:0000011, "product" hierarchy; and if a modifier, the term should be chosen from the SB0:0000019, "modifier" hierarchy. (References: L2V2 Section 4.13.2; L2V3 Sections 4.13 .1 and 5.)

10709. The value of the sboTerm attribute on a KineticLaw must be an SBO identifier referring to a rate law. That is, the value must be a term derived from SB0:0000001, "rate law". (References: L2V2 Section 4.13.5; L2V3 Section 4.13.1.)

10710. The value of the sboTerm attribute on an Event must be an SBO identifier referring to a mathematical expression. That is, the value must be a term derived from SB0:0000231, "event". (References: L2V2 Section 4.14.1; L2V3 Section 4.14.1.)

10711. The value of the sboTerm attribute on an EventAssignment must be an SBO identifier referring to a mathematical expression. That is, the value must be a term derived from SB0:0000064, "mathematical expression". (References: L2V2 Section 4.14.2; L2V3 Section 4.14.1.)

10712. The value of the sboTerm attribute on a Compartment must be an SBO identifier referring to a participant physical type. That is, the value must be a term derived from SB0:0000236,"participant physical type". (References: L2V3 Section 5.)

10713. The value of the sboTerm attribute on a Species must be an SBO identifier referring to a participant physical type. That is, the value must be a term derived from SB0:0000236,"participant physical type". (References: L2V3 Section 5.) 
10714. The value of the sboTerm attribute on a CompartmentType must be an SBO identifier referring to a participant physical type. That is, the value must be a term derived from SB0:0000236, "participant physical type". (References: L2V3 Section 5.)

10715. The value of the sboTerm attribute on a SpeciesType must be an SBO identifier referring to a participant physical type. That is, the value must be a term derived from SB0:0000236, "participant physical type". (References: L2V3 Section 5.)

10716. The value of the sboTerm attribute on a Trigger must be an SBO identifier referring to a mathematical expression. That is, the value must be a term derived from SB0:0000064, "mathematical expression". (References: L2V3 Section 5.)

10717. The value of the sboTerm attribute on a Delay must be an SBO identifier referring to a mathematical expression. That is, the value must be a term derived from SB0:0000064, "mathematical expression". (References: L2V3 Section 5.)

\section{General notes validation}

10801. The contents of the notes element must be explicitly placed in the XHTML XML namespace. (References: L2V3 Section 3.2.3.)

10802. The contents of the notes element must not contain an XML declaration (i.e., a string of the form "<?xml version="1.0" encoding="UTF-8"?>" or similar). (References: L2V2 Section 3.3.2; L2V3 Section 3.2.3.)

10803. The contents of the notes element must not contain an XML DOCTYPE declaration (i.e., a string beginning with the characters "<! DOCTYPE". (References: L2V2 Section 3.3.2; L2V3 Section 3.2.3.)

10804. The XHTML content inside a notes element can only take one of the following general forms: (1) a complete XHTML document beginning with the element $<\mathrm{html}>$ and ending with $</ \mathrm{html}>$; (2) the "body" portion of a document beginning with the element $<$ body $>$ and ending with $</$ body $>$; or (3) XHTML content that is permitted within a $<$ body $>\ldots<$ /body $>$ elements. (References: L2V2 Section 3.3.2; L2V3 Section 3.2.3.)

\section{SBML container validation}

20101. The sbml container element must declare the XML Namespace for SBML, and this declaration must be consistent with the values of the level and version attributes on the sbml element. (References: L2V2 Section 4.1; L2V3 Section 4.1.)

20102. The sbml container element must declare the SBML Level using the attribute level, and this declaration must be consistent with the XML Namespace declared for the sbml element. (References: L2V2 Section 4.1; L2V3 Section 4.1.)

20103. The sbml container element must declare the SBML Version using the attribute version, and this declaration must be consistent with the XML Namespace declared for the sbml element. (References: L2V2 Section 4.1; L2V3 Section 4.1.)

\section{Model validation}

20201. An SBML document must contain a Model definition. (References: L2V1, L2V2 and L2V3 Section 4.1).

20202. The order of subelements within a Model object instance must be the following, with each element optional: list0fFunctionDefinitions, list0fUnitDefinitions, list0fCompartmentTypes, list0fSpeciesTypes, list0fCompartments, list0fSpecies, list0fParameters, list0fInitialAssignments, list0fRules, list0fConstraints, list0fReactions, list0fEvents. (References: L2V2 Section 4.2; L2V3 Section 4.2.) 
20203. The list0f container elements in a Model instance are optional, but if present, the elements must not be empty. Specifically, if any of the following are present in a Model, they must not be empty: list0fFunctionDefinitions, list0fUnitDefinitions, list0fCompartmentTypes, list0fSpeciesTypes, list0fCompartments, list0fSpecies, list0fParameters, list0fInitialAssignments, list0fRules, list0fConstraints, list0fReactions and list0fEvents. (References: This is requirement stemming from the XML Schema used for SBML; L2V3 Section 4.2.)

20204. If a model defines any Species, then the model must also define at least one Compartment. This is an implication of the fact that the compartment attribute on Species is not optional. (References: L2V1 Section 4.5; L2V2 Section 4.8.3; L2V3 Section 4.8.3.)

\section{FunctionDefinition validation}

20301. The top-level element within math in a FunctionDefinition must be one and only one MathML lambda element or a MathML semantics element containing one and only one lambda element. (References: L2V1 Section 4.3.2; L2V2 Section 4.3.2; L2V3 Section 4.3.2.)

20302. Inside the lambda of a FunctionDefinition, if a ci element is the first element within a MathML apply, then the ci's value can only be chosen from the set of identifiers of other SBML FunctionDefinitions defined prior to that point in the SBML model. In other words, forward references to user-defined functions are not permitted. (References: L2V2 Section 3.5.3; L2V3 Section 3.4.3.)

20303. Inside the lambda of a FunctionDefinition, the identifier of that FunctionDefinition cannot appear as the value of a ci element. SBML functions are not permitted to be recursive. (References: L2V2 L2V2 Section 3.5.3 and 4.3.2; L2V3 Sections 3.4.3 and 4.3.2.)

20304. Inside the lambda of a FunctionDefinition, if a ci element is not the first element within a MathML apply, then the ci's value can only be an identifier provided as the value of a bvar element declared in that lambda. This restriction also applies to the csymbol for time. In other words, all model quantities and variables referenced inside a function definition must be passed as arguments to that function. (References: L2V2 L2V2 Section 3.5.3 and 4.3.2; L2V3 Sections 3.4.3 and 4.3.2.)

20305. The value type returned by a FunctionDefinition's lambda must be either boolean or numerical. (References: L2V2 Section 3.5.8; L2V3 Section 3.4.9.)

\section{Unit and UnitDefinition validation}

20401. The value of the id attribute in a UnitDefinition must be of type UnitSId and not be identical to any unit predefined in SBML. That is, the identifier must not be the same as any of the following predefined units: "ampere", "gram", "katal", "metre", "second", "watt", "becquerel", "gray", "kelvin", "mole", "siemens", "weber", "candela", "henry", "kilogram", "newton", "sievert", "coulomb", "hertz", "litre", "ohm", "steradian", "dimensionless", "item", "lumen", "pascal", "tesla", "farad", "joule", "lux", "radian", or "volt". (References: L2V1 erratum 14; L2V2 Section 4.4.2; L2V3 Section 4.4.2.)

20402. Redefinitions of the built-in unit substance must be based on the unit mole, item, gram, kilogram, or dimensionless. More formally, a UnitDefinition for substance must simplify to a single Unit in which either (a) the kind attribute has a value of "mole", "item", "gram", or "kilogram", and the exponent attribute has a value of "1", or (b) the kind attribute has a value of "dimensionless" with any exponent attribute value. (References: L2V1 Section 4.4.3; L2V2 Section 4.4.3; L2V3 Section 4.4.3.)

20403. Redefinitions of the built-in unit length must be based on the unit metre or dimensionless. More formally, a UnitDefinition for length must simplify to a single Unit in which either (a) the kind attribute has a value of "metre" and the exponent attribute has a value of "1", or (b) the kind attribute has a value of "dimensionless" with any exponent value. (References: L2V1 Section 4.4.3; L2V2 Section 4.4.3; L2V3 Section 4.4.3.) 
20404. Redefinitions of the built-in unit area must be based on squared metres or dimensionless. More formally, a UnitDefinition for area must simplify to a single Unit in which either (a) the kind attribute has a value of "metre" and the exponent attribute has a value of " 2 ", or (b) the kind attribute has a value of "dimensionless" with any exponent value. (References: L2V1 Section 4.4.3; L2V2 Section 4.4.3; L2V3 Section 4.4.3.)

20405. Redefinitions of the built-in unit time must be based on the unit second. More formally, a UnitDefinition for time must simplify to a single Unit in which either (a) the kind attribute has a value of "second" and the exponent attribute has a value of "1", or (b) the kind attribute has a value of "dimensionless" with any exponent value. (References: L2V1 Section 4.4.3; L2V2 Section 4.4.3; L2V3 Section 4.4.3.)

20406. Redefinitions of the built-in unit volume must be based on litre, metre or dimensionless. More formally, a UnitDefinition for volume must simplify to a single Unit in which the kind attribute value is either "litre", "metre", or "dimensionless". Additional constraints apply if the kind is "litre" or "metre". (References: L2V1 Section 4.4.3; L2V2 Section 4.4.3; L2V3 Section 4.4.3.)

20407. If a UnitDefinition for volume simplifies to a Unit in which the kind attribute value is "litre", then its exponent attribute value must be "1". (References: L2V1 Section 4.4.3; L2V2 Section 4.4.3; L2V3 Section 4.4.3.)

20408. If a UnitDefinition for volume simplifies to a Unit in which the kind attribute value is "metre", then its exponent attribute value must be "3". (References: L2V1 Section 4.4.3; L2V2 Section 4.4.3; L2V3 Section 4.4.3.)

20409. The list0fUnits container in a UnitDefinition cannot be empty. (References: L2V2 Section 4.4; L2V3 Section 4.4.)

20410. The value of the kind attribute of a Unit can only be one of the predefined base units in SBML; that is, the SBML unit system is not hierarchical and user-defined units cannot be defined using other user-defined units. (References: L2V2 Section 4.4.1; L2V3 Section 4.4.2.)

20411. (Rule does not apply in SBML Level 2 Version 3.)

20412. (Rule does not apply in SBML Level 2 Version 3.)

\section{Compartment validation}

20501. The size of a Compartment must not be set if the compartment's spatialDimensions attribute has value 0. (References: L2V1 Section 4.5.3; L2V2 Section 4.7.4; L2V3 Section 4.7.5.)

20502. If a Compartment definition has a spatialDimensions value of " 0 ", then its units attribute must not be set. If the compartment has no dimensions, then no units can be associated with a non-existent size. (References: L2V1 Section 4.5.4; L2V2 Section 4.7.5; L2V3 Section 4.7.5.)

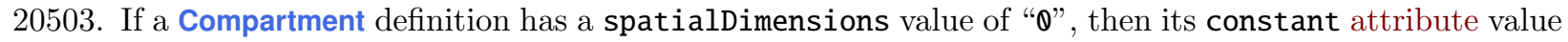
must either default to or be set to "true". If the compartment has no dimensions, then its size can never change. (References: L2V1 Section 4.5.5; L2V2 Section 4.7.4; L2V3 Section 4.7.6.)

20504. The outside attribute value of a Compartment must be the identifier of another Compartment defined in the model. (References: L2V1 Section 4.5.6; L2V2 Section 4.7.7; L2V3 Section 4.7.7.)

20505. A Compartment may not enclose itself through a chain of references involving the outside attribute. This means that a compartment cannot have its own identifier as the value of outside, nor can it point to another compartment whose outside attribute points directly or indirectly to the compartment. (References: L2V1 erratum 11; L2V2 Section 4.7.7; L2V3 Section 4.7.7.) 
20506. The outside attribute value of a given Compartment instance cannot be a compartment having a spatialDimensions value of "0", unless both compartments have spatialDimensions="0". Simply put, a zero-dimensional compartment cannot enclose compartments that have anything other than zero dimensions themselves. (References: L2V2 Section 4.7.7; L2V3 Section 4.7.7.)

20507. The value of the units attribute on a Compartment having spatialDimensions of "1" must be either "length", "metre", "dimensionless", or the identifier of a UnitDefinition based on either metre (with exponent equal to "1") or dimensionless. (References: L2V1 Section 4.5.4; L2V2 Section 4.7.5; L2V3 Section 4.7.5.)

20508. The value of the units attribute on a Compartment having spatialDimensions of " 2 " must be either "area", "dimensionless", or the identifier of a UnitDefinition based on either metre (with exponent equal to "2") or dimensionless. (References: L2V1 Section 4.5.4; L2V2 Section 4.7.5; L2V3 Section 4.7.5.)

20509. The value of the units attribute on a Compartment having spatialDimensions of " 3 " must be either "volume", "litre", or the identifier of a UnitDefinition based on either litre, metre (with exponent equal to "3"), or dimensionless. (References: L2V1 Section 4.5.4; L2V2 Section 4.7.5; L2V3 Section 4.7.5.)

20510. If the compartmentType attribute is given a value in a Compartment definition, it must contain the identifier of an existing CompartmentType defined in the model. (References: L2V2 Section 4.7.2; L2V3 Section 4.7.2.)

\section{Species validation}

20601. The value of compartment in a Species definition must be the identifier of an existing Compartment defined in the model. (References: L2V1 Section 4.6.2; L2V2 Section 4.8.3; L2V3 Section 4.8.3.)

20602. (Rule does not apply in SBML Level 2 Version 3.)

20603. (Rule does not apply in SBML Level 2 Version 3.)

20604. If a Species located in a Compartment whose spatialDimensions is set to "0", then that Species definition cannot set initialConcentration. (References: L2V1 Section 4.6.3; L2V2 Section 4.8.4; L2V3 Section 4.8.4.)

20605. (Rule does not apply in SBML Level 2 Version 3.)

20606. (Rule does not apply in SBML Level 2 Version 3.)

20607. (Rule does not apply in SBML Level 2 Version 3.)

20608. The value of a Species's substanceUnits attribute can only be one of the following: "substance", "mole", "item", "gram", "kilogram", "dimensionless", or the identifier of a UnitDefinition derived from "mole" (with an exponent of "1"), "item" (with an exponent of "1"), "gram" (with an exponent of "1"), "kilogram" (with an exponent of "1"), or "dimensionless". (References: L2V1 Section 4.6.4; L2V2 Section 4.8.5; L2V3 Section 4.8.5.)

20609. A Species cannot set values for both initialConcentration and initialAmount because they are mutually exclusive. (References: L2V1 Section 4.6.3; L2V2 Section 4.8.4; L2V3 Section 4.8.4.)

20610. A Species' quantity cannot be determined simultaneously by both reactions and rules. More formally, if the identifier of a Species definition having boundaryCondition="false" and constant="false" is referenced by a SpeciesReference anywhere in a model, then this identifier cannot also appear as the value of a variable in an AssignmentRule or a RateRule. (References: L2V1 Section 4.6.5; L2V2 Section 4.8.6; L2V3 Section 4.8.6.) 
20611. A Species having boundaryCondition="false" cannot appear as a reactant or product in any reaction if that Species also has constant="true". (References: L2V1 Section 4.6.5; L2V2 Section 4.8.6; L2V3 Section 4.8.6.)

20612. The value of speciesType in a Species definition must be the identifier of an existing SpeciesType. (References: L2V2 Section 4.8.2; L2V3 Section 4.8.2.)

20613. There cannot be more than one species of a given SpeciesType in the same compartment of a model. More formally, for any given compartment, there cannot be more than one Species definition in which both of the following hold simultaneously: (i) the Species' compartment value is set to that compartment's identifier and (ii) the Species' speciesType is set the same value as the speciesType of another Species that also sets its compartment to that compartment identifier. (References: L2V2 Section 4.8.2; L2V3 Section 4.8.2.)

20614. The compartment attribute in a Species is mandatory. A species definition in a model must include a value for this attribute. (References: L2V2 Section 4.8.3; L2V3 Section 4.8.3.)

\section{Parameter validation}

20701. The units in a Parameter definition must be a value chosen from among the following: a built-in unit (e.g., "substance", "time", etc.), the identifier of a UnitDefinition in the model, or one of the predefined base units in SBML (e.g., "litre", "mole", "metre", etc.). (References: L2V1 Section 4.7.3; L2V2 Section 4.9.3; L2V3 Section 4.9.3.)

\section{InitialAssignment validation}

20801. The value of symbol in an InitialAssignment definition must be the identifier of an existing Compartment, Species, or Parameter defined in the model. (References: L2V2 Section 4.10.1; L2V3 Section 4.10.)

20802. A given identifier cannot appear as the value of more than one InitialAssignment's symbol attribute across the set of all InitialAssignments in a model. (References: L2V2 Section 4.10; L2V3 Section 4.10.)

20803. The identifier given as the value of a symbol attribute in any InitialAssignment definition cannot also appear as the value of the variable attribute in an AssignmentRule. In other words, a model cannot simultaneously define both an initial assignment and an assignment rule for the same species, compartment or parameter in a model. (References: L2V2 Section 4.10.4; L2V3 Section 4.10.)

\section{AssignmentRule and RateRule validation}

20901. The value of an AssignmentRule's variable must be the identifier of an existing Compartment, Species, or globally-defined Parameter. (References: L2V1 Section 4.8.2; L2V2 Section 4.11.3 L2V3 Section 4.11.3.)

20902. The value of a RateRule's variable must be the identifier of an existing Compartment, Species, or globally-defined Parameter. (References: L2V1 Section 4.8.3; L2V2 Section 4.11.4; L2V3 Section 4.11.4.)

20903. Any Compartment, Species or Parameter whose identifier is the value of a variable attribute in an AssignmentRule, must have a value of "false" for constant. (References: L2V1 Section 4.8.4; L2V2 Section 4.11.3; L2V3 Section 4.11.3.)

20904. Any Compartment, Species or Parameter whose identifier is the value of a variable attribute in a RateRule, must have a value of "false" for constant. (References: L2V1 Section 4.8.4; L2V2 Section 4.11.4; L2V3 Section 4.11.4.)

20905. (Rule removed because it was effectively a duplicate of 10304.) 
20906. There must not be circular dependencies in the combined set of InitialAssignment, AssignmentRule and KineticLaw definitions in a model. Each of these constructs has the effect of assigning a value to an identifier (i.e., the identifier given in the attribute symbol in InitialAssignment, the attribute variable in AssignmentRule, and the attribute id on the KineticLaw's enclosing Reaction). Each of these constructs computes the value using a mathematical formula. The formula for a given identifier cannot make reference to a second identifier whose own definition depends directly or indirectly on the first identifier. (References: L2V2 Section 4.11.5; L2V3 Section 4.11.5.)

\section{Constraint validation}

21001. A Constraint math expression must evaluate to a value of type boolean. (References: L2V2 Section 4.12.1; L2V3 Section 4.12.)

21002. The order of subelements within Constraint must be the following: math, message. The message element is optional, but if present, must follow the math element. (References: L2V2 Section 4.12; L2V3 Section 4.12.)

21003. The contents of the message element in a Constraint must be explicitly placed in the XHTML XML namespace. (References: L2V3 Section 4.12.2.)

21004. The contents of the message element must not contain an XML declaration (i.e., a string of the form "<?xml version="1.0" encoding="UTF-8"?>" or similar). (References: L2V3 Section 4.12.2.)

21005. The contents of the message element must not contain an XML DOCTYPE declaration (i.e., a string beginning with the characters "<! DOCTYPE". (References: L2V3 Section 4.12.2.)

21006. The XHTML content inside a Constraint's message element can only take one of the following general forms: (1) a complete XHTML document beginning with the element $<\mathrm{html}>$ and ending with $</$ xhtml $>$; (2) the "body" portion of a document beginning with the element <body $>$ and ending with $<$ /body $>$; or (3) XHTML content that is permitted within a $<$ body $>\ldots<$ /body $>$ elements. (References: L2V3 Section 4.12.2.)

\section{Reaction validation}

21101. A Reaction definition must contain at least one SpeciesReference, either in its list0fReactants or its list0fProducts. A reaction without any reactant or product species is not permitted, regardless of whether the reaction has any modifier species. (References: L2V2 Section 4.13.1; L2V3 Section 4.13.3.)

21102. The order of subelements within Reaction must be the following (where every one is optional): list0fReactants, list0fProducts, list0fModifiers, kineticLaw. (References: L2V2 Section 4.13; L2V3 Section 4.13.)

21103. The following containers are all optional in a Reaction, but if any present is, it must not be empty: list0fReactants, list0fProducts, list0fModifiers, kineticLaw. (References: L2V2 Section 4.13; L2V3 Section 4.13.)

21104. The list of reactants (list0fReactants) and list of products (list0fProducts) in a Reaction can only contain speciesReference elements. (References: L2V1 Section 4.9; L2V2 Section 4.13; L2V3 Section 4.13.)

21105. The list of modifiers (list0fModifiers) in a Reaction can only contain modifierSpeciesReference elements. (References: L2V1 Section 4.9; L2V2 Section 4.13; L2V3 Section 4.13.)

\section{SpeciesReference and ModifierSpeciesReference validation}

21111. The value of a SpeciesReference species attribute must be the identifier of an existing Species in the model. (References: L2V1 Section 4.9.5; L2V2 Section 4.13.2; L2V3 Section 4.13.3.) 
21112. (Rule removed because it was effectively a duplicate of 20611.)

21113. A SpeciesReference must not have a value for both stoichiometry and stoichiometryMath; they are mutually exclusive. (References: L2V1 Section 4.9.5; L2V2 Section 4.13.3; L2V3 Section 4.13.3.)

\section{KineticLaw validation}

21121. All species referenced in the KineticLaw formula of a given reaction must first be declared using SpeciesReference or ModifierSpeciesReference. More formally, if a Species identifier appears in a ci element of a Reaction's KineticLaw formula, that same identifier must also appear in at least one SpeciesReference or ModifierSpeciesReference in the Reaction definition. (References: L2V2 Section 4.13.5; L2V3 Section 4.13.5.)

21122. The order of subelements within KineticLaw must be the following: math, list0fParameters. The list0fParameters is optional, but if present, must follow math. (References: L2V2 Section 4.13.5; L2V3 Section 4.13.5.)

21123. If present, the list0fParameters in a KineticLaw must not be an empty list. (References: L2V2 Section 4.13.5; L2V3 Section 4.13.)

21124. The constant attribute on a Parameter local to a KineticLaw cannot have a value other than "true". The values of parameters local to KineticLaw definitions cannot be changed, and therefore they are always constant. (References: L2V2 Section 4.9.4; L2V3 Section 4.9.4.)

21125. (Rule does not apply in SBML Level 2 Version 3.)

21126. (Rule does not apply in SBML Level 2 Version 3.)

\section{StoichiometryMath validation}

21131. All species referenced in the StoichiometryMath formula of a given reaction must first be declared using SpeciesReference or ModifierSpeciesReference. More formally, if a Species identifier appears in a ci element of a Reaction's StoichiometryMath formula, that same identifier must also appear in at least one SpeciesReference or ModifierSpeciesReference in the Reaction definition. (References: L2V2 Sections 4.13.2 and 4.13.4; L2V3 Sections 4.13.2 and 4.13.4.)

\section{Event validation}

21201. An Event object must have a trigger. (References: L2V1 Section 4.10.2; L2V2 Section 4.14.1; L2V3 Section 4.14.2.)

21202. An Event trigger expression must evaluate to a value of type boolean. (References: L2V1 Section 4.10.2; L2V2 Section 4.14.1; L2V3 Section 4.14.2.)

21203. An Event object must have at least one EventAssignment object in its list0fEventAssignments. (References: L2V1 Section 4.10.5; L2V2 Section 4.14; L2V3 Section 4.14.)

21204. (Rule does not apply in SBML Level 2 Version 3.)

21205. The order of subelements within an Event object instance must be the following: trigger, delay, list0fEventAssignments. The delay element is optional, but if present, must follow trigger. (References: L2V2 Section 4.14; L2V3 Section 4.14.)

\section{EventAssignment validation}

21211. The value of variable in an EventAssignment can only be the identifier of a Compartment, Species, or model-wide Parameter definition. (References: L2V1 Section 4.10.5; L2V2 Section 4.14.2; L2V3 Section 4.14.4.) 
21212. Any Compartment, Species or Parameter definition whose identifier is used as the value of variable in an EventAssignment must have a value of "false" for its constant attribute. (References: L2V1 Section 4.10.5; L2V2 Section 4.14.2; L2V3 Section 4.14.4.) 


\section{A method for assessing whether an SBML model is overdetermined}

As explained in Section 4.11.5, an SBML model must not be overdetermined. It is possible to use purely static analysis to assess this condition for the system of equations implied by a model, by constructing a bipartite graph of the model's variables and equations and then searching for a maximal matching (Chartrand, 1977). A efficient algorithm for finding a maximal matching is described by Hopcroft and Karp (1973). In this appendix, we provide a concrete application to SBML of the general approach described in Section 4.11.5. The approach is defined in terms of the ordinary differential equations (ODEs) implied by an SBML model; despite our use of a differential equation framework for this explanation, it should be understood that this use of ODEs has no implication about the framework actually used to simulate the model.

\section{Definition of the method}

First, we treat both assignment rules and formulas in KineticLaw objects as SBML-style algebraic equations. (Both are essentially assignment statements, and assignment statements can be trivially converted to algebraic equations with zero on the left-hand side.) We also assume that an ODE is constructed for each species determined by one or more Reaction's KineticLaw math expressions. Finally, we assume that the model has already been determined to be valid in all other respects (e.g., there are no undefined variables in the equations), and what remains is to evaluate whether it is overdetermined.

We construct the bipartite graph for a given SBML model as follows:

1. For each of the following in the model, create one vertex representing an equation:

(a) Every Species object having boundaryCondition="false", constant="false", and which is referenced as a reactant or product in one or more Reaction objects containing KineticLaw objects

(b) Every AssignmentRule object

(c) Every RateRule object

(d) Every AlgebraicRule object

(e) Every KineticLaw object

2. For each of the following in the model, create one vertex representing a variable:

(a) Every Species object having constant= "false"

(b) Every Compartment object having constant="false"

(c) Every global Parameter having constant="false"

(d) Every Reaction object

3. For each of the following, create one edge:

(a) Every vertex created in step 2(a) to that species' equation vertex created in step 1(a)

(b) Every vertex created in step 1(b) to the particular vertex created in steps 2(a)-2(d) that represents the variable referenced by the variable attribute of the rule

(c) Every vertex created in step 1(c) to the particular vertex created in steps 2(a)-2(d) that represents the variable referenced by the variable attribute of the rule

(d) Every vertex created in step 1(e) to the particular vertex created in step 2(d) that is the Reaction object containing that particular KineticLaw object

(e) Every vertex created in steps 2(a)-2(d) representing an identifier appearing as the content of a MathML ci element within an expression of an AssignmentRule, to the vertex for that particular AssignmentRule created in step 1(b)

(f) Every vertex created in steps 2(a)-2(d) representing an identifier appearing as the content of a MathML ci element within an expression of an AlgebraicRule, to the vertex for that particular AlgebraicRule created in step $1(\mathrm{~d})$

(g) Every vertex created in steps 2(a)-2(c) representing an identifier appearing as the content of a MathML ci element within an expression of a KineticLaw, to the vertex for that particular KineticLaw created in step 1(e) 


\section{Example application of the method}

What follows is an example of applying the method above to the SBML model shown below:

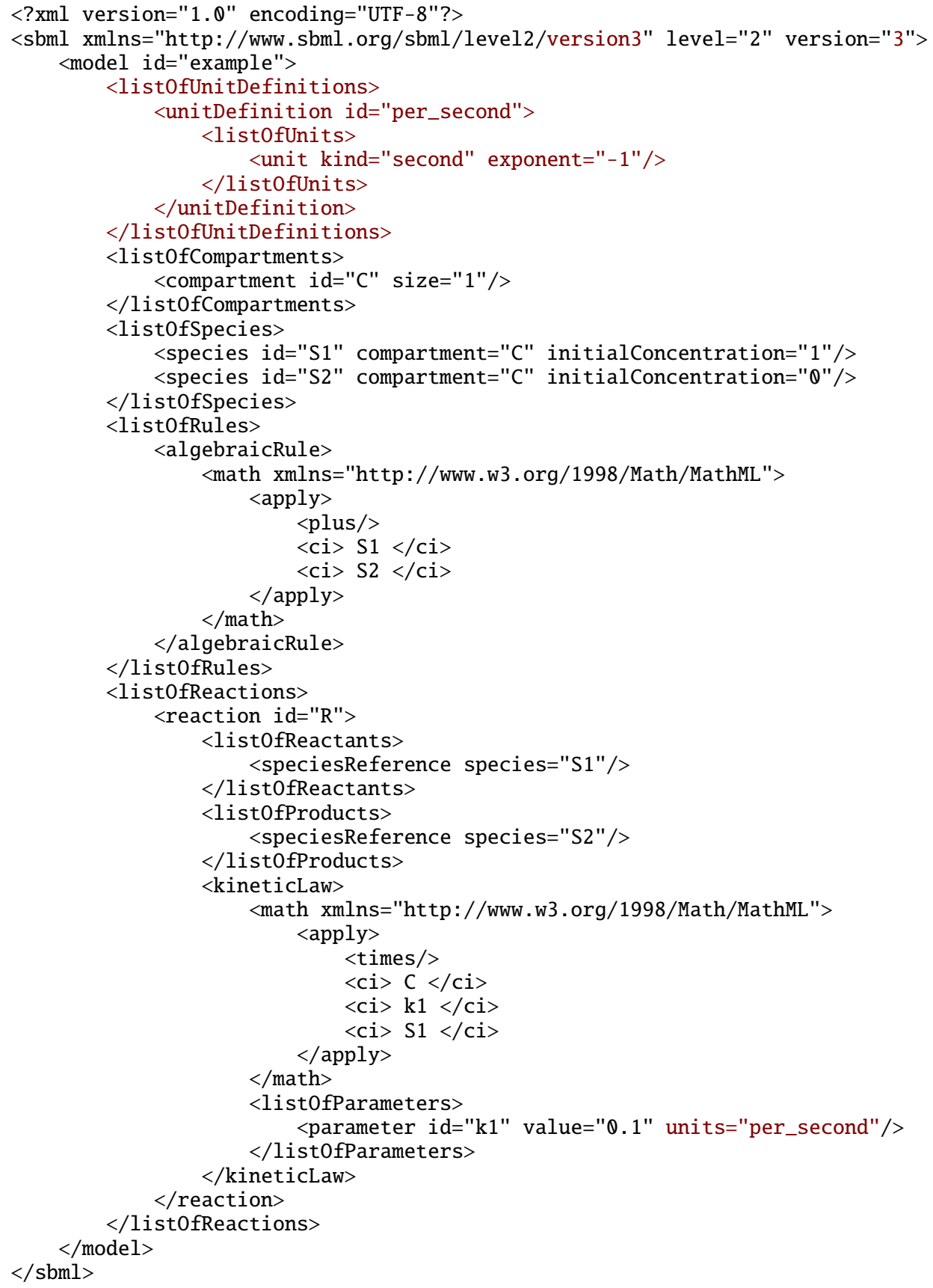

For the model above, we create equation vertices as follows:

1. [Corresponding to step 1(a) in Section D.] Every Species object having boundaryCondition= "false", constant $=$ "false", and which is referenced as a reactant or product in one or more Reaction objects containing KineticLaw objects. This generates two vertices, for "S1" and "S2".

2. [Corresponding to step 1(b) in Section D.] Every AlgebraicRule object. This generates one vertex, for the model's lone algebraic rule (call it "rule").

3. [Corresponding to step 1(e) in Section D.] Every KineticLaw object. This generates one vertex, for the lone kinetic law in the model (call it "law"). 
We create variable vertices for the following:

1. [Corresponding to step 2(a) in Section D.] Every Species object having constant $=$ "false". This generates two vertices, for "S1" and "S2".

2. [Corresponding to step 2(b) in Section D.] Every Compartment object having constant $=$ "false". This generates one vertex, for "C".

3. [Corresponding to step 2(d) in Section D.] Every Reaction object. This generates one vertex, for "R".

Note that it is not necessary to include parameters declared within KineticLaw objects because they are local to a particular reaction and cannot be affected by rules. With the steps above, we have the following set of graph nodes:

\section{Vertices for equations}

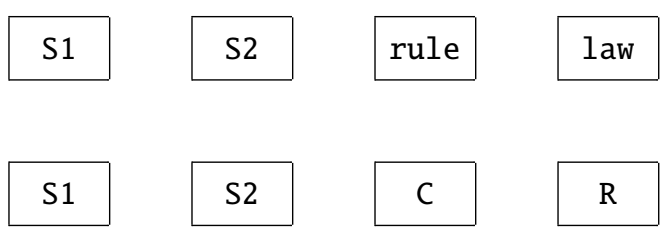

Vertices for variables

Next, we create edges following the procedure described above. Doing so results in the following graph:

Vertices for equations

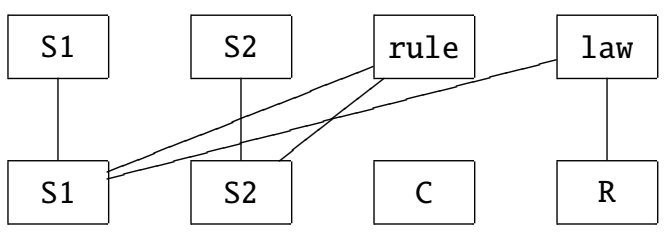

Vertices for variables

The algorithm of Hopcroft and Karp (1973) can now be applied to search for a maximal matching of the bipartite graph. A maximal matching is a graph in which each vertex is connected to at most one other vertex and the maximum possible number of connections have been made. Doing so here results in the following:

\section{Vertices for equations}

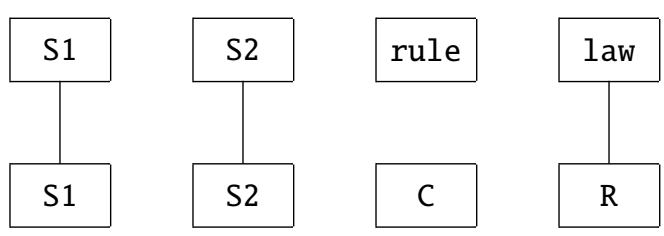

Vertices for variables

If the maximal matching of the bipartite graph leaves any equation vertex unconnected, then the model is considered overdetermined. That is the case for the example shown here, because the equation vertex for "rule" is unconnected in the maximal matching. 


\title{
E Mathematical consequences of the fast attribute on Reaction
}

\author{
(Appendix contributed by James C. Schaff, University of Connecticut Health Center, Connecticut, U.S.A.)
}

Section 4.13.1 described the fast flag available on Reaction. In this appendix, we discuss the principles involved in interpreting this attribute in the context of a simple biochemical reaction model. The derivation presented here is not fully rigorous and this section is not considered normative; achieving a higher level of rigor would require considerably more background exposition and a much longer appendix. Nevertheless, we hope this section is sufficient to answer unambiguously the question "How should a system of reactions be treated if some of the reactions have fast=true?"

\section{Identification of "fast" reactions}

First, it is worth noting that the identification of so-called fast reactions is actually a modeling issue, not an SBML representation issue. The notion of fast reactions is the following. A system may be decomposable into two sets of reactions, where one set may have characteristic times that are much faster than the other time scales in the system. An approximation that is sometimes useful is to assume that the fast reactions have kinetics that settle infinitely fast compared to the other reactions in the system. In other words, the fast reactions are assumed to be always in equilibrium. This is called a pseudo-steady state approximation (PSSA), and is also known as a quasi-steady state approximation (QSSA). Given a case where the time-scale separation between fast and other reactions in the system is large, an accurate and efficient approach for computing the time-course of the system behavior is to treat the fast reactions as being always in equilibrium.

The key to successful application of a PSSA is that there should be a significant separation of time scales between these fast reactions and other reactions in the system. The determination of which reactions qualify as fast is up to the creator of the model, because there is currently no known general algorithm for doing so.

\section{Simple one-compartment biochemical system model}

To explain how to solve a system containing fast reactions, we use a simple model of a biochemical reaction network located in a single compartment. Let $\mathbf{x}^{*}$ represent a vector of all the species in the system, $\mathbf{v}^{*} \mathbf{a}$ vector of the reaction rates, and $\mathbf{A}^{*}$ the stoichiometry matrix, with the vector dimension being $\mathbf{n}^{*}$. Then the system can be described using the following matrix equation:

$$
\frac{d \mathbf{x}^{*}}{d t}=\mathbf{A}^{*} \mathbf{v}^{*}\left(\mathbf{x}^{*}\right)
$$

This system can be optionally reduced by noting that mass conservation usually implies there are linear combinations of species quantities in the system and the value of these combinations do not change over time. Identifying these combinations is the topic of structural analysis and is described in the literature (Reder, 1988; Sauro and Ingalls, 2003). Briefly, let $\mathbf{N}$ be defined as the left null space of $\mathbf{A}^{*}$ :

$$
\mathbf{N A}^{*}=\mathbf{0}
$$

Now, premultiply the previous equation by $\mathbf{N}$ to get

$$
\mathbf{N} \frac{d \mathbf{x}^{*}}{d t}=\mathbf{N A}^{*} \mathbf{v}^{*}\left(\mathbf{x}^{*}\right)=\mathbf{0}
$$

Thus, $\mathbf{N}$ captures the space of solutions to the equation

$$
\mathbf{m}^{T}\left(\frac{d \mathbf{x}^{*}}{d t}\right)=0
$$

where $\mathbf{m}$ is a vector representing the coefficients in a mass conservation relationship, that is, combinations of species that are time-invariant. Now, let

$$
\begin{aligned}
& r=\operatorname{rank}\left(\mathbf{A}^{*}\right) \\
& n=\operatorname{dim}\left(\mathbf{x}^{*}\right)
\end{aligned}
$$


Then the system has $n-r$ mass conservation relationships, each of which is a linear equation. We can use these $n-r$ linear equations to solve for $n-r$ dependent variables in terms of $r$ independent variables and the initial masses of all species. Doing that allows us to decompose $\mathbf{x}^{*}$ into $n-r$ dependent variables $\mathbf{x}_{d}$ and $r$ independent variables $\mathbf{x}_{i}$ where $\mathbf{L}$ is an $(n-r) \times r$ matrix that is derived from $\mathbf{N}$ and represents $\mathbf{x}_{d}$ in terms of $\mathbf{x}_{i}, \mathbf{I}$ is the $r \times r$ identity matrix, and $\mathbf{T}$ is an $n \times r$ matrix:

$$
\mathbf{x}^{*} \equiv\left[\begin{array}{l}
\mathbf{x}_{i} \\
\mathbf{x}_{d}
\end{array}\right]=\left[\begin{array}{l}
\mathbf{I} \\
\mathbf{L}
\end{array}\right] \mathbf{x}_{i}=\mathbf{T} \mathbf{x}_{i}
$$

Using this equation, we can define a new vector of reaction velocities $\mathbf{v}$ in terms of $\mathbf{x}_{i}$ only:

$$
\mathbf{v}\left(\mathbf{x}_{i}\right) \equiv \mathbf{v}^{*}\left(\mathbf{T x}_{i}\right)
$$

With this $\mathbf{v}$, we can now write a reduced system by substituting terms. First we define $\mathbf{A}$ as the $r$ independent rows of $\mathbf{A}^{*}$ corresponding to $\mathbf{x}_{i}$. Then:

$$
\frac{d \mathbf{x}_{i}}{d t}=\mathbf{A} \mathbf{v}\left(\mathbf{x}_{i}\right)
$$

This is a set of $r$ independent differential equations in $r$ unknowns (i.e., an $r$-dimensional system). To simplify the notation slightly, let

$$
\mathbf{x} \equiv \mathbf{x}_{i}
$$

and, thus,

$$
\frac{d \mathbf{x}}{d t}=\mathbf{A v}(\mathbf{x})
$$

\section{Application of a PSSA to biochemical systems}

Assume that we have eliminated redundant variables and equations using the mass conservation analysis above. Further assume that we have some external means of classifying some reactions in a given system as being fast as discussed earlier. We now need to apply this to the system under study. We begin by decomposing the vector of reaction velocities $\mathbf{v}$ according to fast and slow reactions:

$$
\frac{d \mathbf{x}}{d t}=\mathbf{A}_{1} \mathbf{v}_{f}(\mathbf{x})+\mathbf{A}_{2} \mathbf{v}_{s}(\mathbf{x})
$$

In the expression above, $\mathbf{A}_{1}$ represents the stoichiometry of the set of reactions operating on the fast time scale, and $\mathbf{A}_{2}$ the stoichiometry of the set of reactions operating on a slower time scale. We find the left null space of $\mathbf{A}_{1}$ (i.e., the space of solutions to $\mathbf{m}^{T}[d \mathbf{x} / d t]=0$ on a fast time scale), and call this matrix $\mathbf{B}$ :

$$
\mathbf{B A}_{1}=\mathbf{0}
$$

The matrix $\mathbf{B}$ represents the linear combination of species that do not change on a fast time scale, i.e., the slow species in the system. Now, we premultiply the equation for $d \mathbf{x} / d t$ by $\mathbf{B}$ :

$$
\begin{aligned}
\mathbf{B} \frac{d \mathbf{x}}{d t} & =\mathbf{B A}_{1} \mathbf{v}_{f}(\mathbf{x})+\mathbf{B} \mathbf{A}_{2} \mathbf{v}_{s}(\mathbf{x}) \\
& =\mathbf{B A}_{2} \mathbf{v}_{s}(\mathbf{x})
\end{aligned}
$$

where the second line follows from the fact that $\mathbf{B} \mathbf{A}_{1}=\mathbf{0}$. The above is an ordinary differential equation in terms of only the slow dynamics. The remaining fast dynamics are handled by applying the pseudo-steady state approximation, with fast transients assumed to have settled with respect to the slow time scale. This produces a system of nonlinear algebraic equations:

$$
\mathbf{A}_{1} \mathbf{v}_{f}=\mathbf{0}
$$

The last two equations form the system of equations resulting from the application of the PSSA. If $r_{1}=$ $\operatorname{rank}\left(\mathbf{A}_{1}\right)$ and $r=\operatorname{rank}(\mathbf{A})$, then there will be $r_{1}$ degrees of freedom that will be determined by solving an algebraic system (the equation $\mathbf{A}_{1} \mathbf{v}_{f}=\mathbf{0}$ above), and there will be $r-r_{1}$ degrees of freedom that will be determined by ordinary differential equations (the equation for $\mathbf{B} d \mathbf{x} / d t$ ). 


\section{F Processing and validating SBase notes and Constraint message content}

In Section 3.2.3 and Section 4.12.2, we discussed the notes element on Sbase and the message element on Constraint, respectively. These elements can contain a number of possible forms of XHTML content. In this appendix, we describe a general procedure for how application software can process such content. We concentrate on the common case of an SBML-reading application that needs to take the contents and pass it to an XHTML display and/or editing function obtained from a third-party API library. The content of the notes and message may not be a complete XHTML document, so the application will have to perform some processing before handing it to the XHTML editor or validator. How should this be done?

Based on the three forms of Sbase notes content described in Section 3.2.3 and the identical forms for Constraint message described in Section 4.12.2, there are only three cases possible. Here we give an example approach for handling them, although the actual implementation details will differ depending on various factors such as the requirements of the software libraries being used. This example approach would be performed for each notes and message to be viewed or edited:

Step 1. If the XHTML viewing/editing function requires a fully compliant XML document, the SBML application could create a temporary data object containing an appropriate XML declaration and a DOCTYPE declaration; otherwise, the XML data object can be initially blank.

Step 2. The application should look at the first element inside the notes or message (or rather, the first element that is not an XML comment), and take action based on the following three possibilities:

- If the first element begins with $<\mathrm{html}$ xhtml="http://www.w3.org/1999/xhtml" $>$, the application could assume that the content is a complete XHTML document and insert this into the temporary data object.

- Else, if the first element is $<$ body $>$, the application should insert the following into the temporary data object,

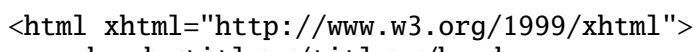

then insert the content of the notes or message, and finally insert a closing $</ \mathrm{html}>$.

- Else, if the content begins with neither of the above elements, the application should insert the following into the temporary data object,

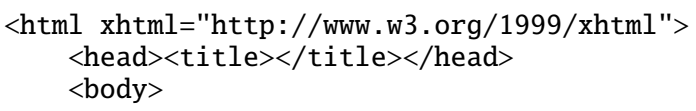

then insert the content of the notes or message, and finally insert $</$ body $></$ html $>$ to close the XHTML document.

The result of the above would be a temporary XML data object that the application could then pass to the XHTML viewing/editing API function. 


\section{G Major changes between versions of SBML Level 2 and implications for back- ward compatibility}

In Section 1.3, we summarized the language features removed and deprecated in SBML Level 2 Version 3. Here we describe the changes in more detail. We describe the cumulative changes introduced since SBML Level 2 Version 1.

\section{G.1 Between Version 2 and Version 1}

The following features were removed between SBML Level 2 Version 1 and Version 2:

- The offset attribute on UnitDefinition. (See Section 4.4.2.) The definition of offsets in SBML Level 2 Version 1 was in fact incorrect; moreover, a proper implementation would have required a complete change in the SBML unit scheme. Few models appeared to use offsets on unit definitions, so the impact of this change on models is expected to be small.

- The "Celsius" predefined unit. (See Section 4.4.2.) The removal of offsets on unit definitions meant an inconsistency existed if the Celsius predefined unit was left in the system. Removing Celsius removes the inconsistency. Alternative ways of using Celsius are discussed in Section 4.4.2.

- The substanceUnit and timeUnits attributes on KineticLaw. (See Section 4.13.5.) The ability to redefine the substance units on each reaction separately, coupled with other features in SBML Level 2 Version 1, created the opportunity for defining a valid system of reactions which potentially could not be combined into a consistent system of equations without external knowledge.

The following features were deprecated between SBML Level 2 Version 1 and Version 2:

- The charge attribute on Species. (See Section 4.8.7.) This attribute does not appear to be supported by any existing software, and moreover, since its value cannot be accessed from mathematical formulas in SBML, the impact of this change is expected to be small.

The following additional changes were made between SBML Level 2 Version 1 and Version 3:

- SBML Level 2 Version 1 did not clearly specify the value space of integer and floating-point numbers permitted in the MathML expressions in SBML; moreover, it used the XML Schema type "integer" instead of SBML Level 2 Version 2's "int". Although extremely unlikely, some previously valid SBML Level 2 Version 1 documents may not be valid in Version 2 and Version 3 as a result of these changes. See Sections 3.1.3, 3.1.5 and 3.4.2 for more information.

- SBML Level 2 Version 1 did not define a default value for the attribute fast on Reaction. SBML Level 2 Version 2 introduced a default value, and the value is "false". Further, SBML now requires that software tools must respect the value or indicate to the user that they do not have the capacity to do so. See Section 4.13.1.

- As of SBML Level 2 Version 2, SBML is somewhat stricter about how the content of annotation elements must be organized and written.. Previously valid SBML Level 2 Version 1 documents may need changes to their annotation elements to comply with the specification beginning with Version 2 and Version 3. See Section 3.2.4 for more details.

- As of SBML Level 2 Version 2, SBML is slightly stricter about how the content of notes elements must be organized. Previously valid SBML Level 2 Version 1 documents may need changes to their notes elements to comply with the specification beginning with Version 2 and Version 3. See Section 3.2.3 for more details.

- SBML Level 2 Version 2 corrected numerous errata and ambiguities discovered in SBML Level 2 Version 1. These errata are listed on the project web site at http://sbml.org. As a result of changes 
to SBML Level 2 implied by these errata, some existing SBML Level 2 Version 1 models, even when modified as explained above, may still not be compliant with Version 2 or Version 3. The ultimate impact of the changes depends on the specific features used by a given model and the assumptions under which the model was created.

\section{G.2 Between Version 3 and Version 2}

The following features were removed between SBML Level 2 Version 2 and Version 3:

- The spatialSizeUnits attribute on Species. (See Section 4.8.5.) This attribute introduced an implicit unit conversion between the spatial units used in defining the quantity of a species and the size of the compartment in which the species was located. Moreover, the semantics of spatialSizeUnits were confusing and required complicated unit conversions to be written explicitly into reaction rate expressions by either the modeler or the software. Although the conversions could be worked out unambiguously, the potential for error was judged to exceed by far the utility of this feature.

- The timeUnits attribute Event. (See Section 4.14.) This attribute was judged to add needless complexity and inconsistency. For instance, the ability to change the time units of the delay of an Event to be different from the units of time for the whole model meant that computing an Event's time of triggering and its delay might have to be done using two different sets of units. The ability to redefine the units of time for the delay of an Event was also inconsistent with the lack of such an attribute on other SBML components involving an element of time; for example, RateRule, and now KineticLaw, have no such attributes.

The following additional changes were made between SBML Level 2 Version 2 and Version 3:

- The definition of the XML type ID was incorrectly given in the SBML Level 2 Version 2 specification. This type is used as the type of the attribute metaid on Sbase. The error in the definition of ID was such that the type did not include the colon (:) character and all Unicode characters actually permitted in XML ID. This change is therefore entirely backward compatible: all models with valid metaid values valid prior to SBML Level 2 Version 3 are still valid under the new definition.

- The SBML specifications prior to SBML Level 2 Version 3 did not indicate what units are assumed for literal numbers appearing in MathML expressions (i.e., numbers inside MathML's cn elements). SBML Level 2 Version 3 stipulates that there are no units associated with numbers (not even "dimensionless"), and provides suggestions for how to associate units with numbers (Section 3.4.2).

- The SBML specifications prior to SBML Level 2 Version 3 did not make clear what units are required by the arguments to various MathML operators and other constructs. SBML Level 2 Version 3 clarifies this (Section 3.4.10).

- The UnitKind enumeration previously defined in the context of Unit and UnitDefinition has been eliminated in favor of simply defining the symbols as reserved words in the value space of UnitSId. This has no effect on written models and is completely backward compatible. It was done to resolve the problem that, previously, the values in UnitKind were technically inaccessible from attributes whose data type was UnitSId.

- The SBML specification did not point out that the value space of the data type boolean is different in MathML 2.0 and XML Schema 1.0. This means that the permitted values of attributes on SBML objects is different from the values permitted in MathML formulas. (Section 3.1.2 explains the difference.)

- The SBML specifications were inconsistent about the permitted number of items inside list0f

lists: the text portion of the specifications claimed the lists could have zero length, but the XML Schema definition for SBML required one or more items. As of SBML Level 2 Version 3, the specification is consistent on requiring one or more items inside these lists. 
- The SBO term hierarchy (Section 5) has grown in the time intervening between SBML Level 2 Version 2 and Version 3, and the mapping of terms between SBO and SBML components was revised as a result of community discussions during the 2006 SBML Forum meeting.

- The sboTerm attribute, introduced on many components in SBML Level 2 Version 2, has been moved to Sbase as an optional attribute and removed from the individual components. The result is that all model components may now have SBO terms associated with them. This change is completely backward compatible.

- A number of validation rules in Appendix $\mathrm{C}$ have been introduced; some were missing from previous specifications, and some were added to cover changes introduced in SBML Level 2 Version 3 (for example, for validation of SBO terms assigned to various SBML model components).

- The SBML specifications prior to SBML Level 2 Version 3 did not adequately explain the assumptions regarding XML namespace declarations within the annotation element on SBML components. SBML Level 2 Version 3 makes these assumptions more explicit, including the assumption that applications may not preserve another applications annotations unless those annotations are self-contained with the XML Namespace declaration. See Section 3.2.4 for more details. 


\section{References}

Abbott, A. (1999). Alliance of US labs plans to build map of cell signalling pathways. Nature, 402:219-200.

Abramowitz, M. and Stegun, I. A., editors (1977). Mathematical Fuctions: With Formulas, Graphs, and Mathematical Tables. Dover Publications Inc.

Ausbrooks, R., Buswell, S., Carlisle, D., Dalmas, S., Devitt, S., Diaz, A., Froumentin, M., Hunter, R., Ion, P., Kohlhase, M., Miner, R., Poppelier, N., Smith, B., Soiffer, N., Sutor, R., and Watt, S. (2003). Mathematical Markup Language (MathML) Version 2.0 (second edition): W3C Recommendation 21 October 2003. Available via the World Wide Web at http://www.w3.org/TR/2003/REC-MathML2-20031021/.

Biron, P. V. and Malhotra, A. (2000). XML Schema part 2: Datatypes (W3C candidate recommendation 24 October 2000). Available via the World Wide Web at http://www.w3.org/TR/xmlschema-2/.

Bosak, J. and Bray, T. (1999). XML and the second-generation Web. Scientific American, 280(5):89-93.

Bray, T., D. Hollander, D., and Layman, A. (1999). Namespaces in XML. World Wide Web Consortium 14-January-1999. Available via the World Wide Web at http://www.w3.org/TR/1999/ REC-xml-names-19990114/.

Bray, T., Paoli, J., Sperberg-McQueen, C. M., and Maler, E. (2000). Extensible markup language (XML) 1.0 (second edition), W3C recommendation 6-October-2000. Available via the World Wide Web at http: //www.w3.org/TR/1998/REC-xml-19980210.

Bray, T., Paoli, J., Sperberg-McQueen, C. M., Maler, E., and Yergeau, F. (2004). Extensible markup language (XML) 1.0 (third edition), W3C recommendation 4-February-2004. Available via the World Wide Web at http://www.w3.org/TR/2004/REC-xml-20040204.

Bureau International des Poids et Mesures (2000). The International System of Units (SI) supplement 2000: addenda and corrigenda to the 7th edition (1998). Available via the World Wide Web at http: //www.bipm.fr/pdf/si-supplement2000.pdf.

Chartrand, G. (1977). Introductory Graph Theory. Dover Publishing, Inc., New York.

DCMI Usage Board (2005). DCMI Metadata Terms. Available via the World Wide Web at http://www. dublincore. org/documents/dcmi-terms/.

Dublin Core Metadata Initiative (2005). Dublin core metadata initiative. Available via the World Wide Web at http://dublincore.org/.

Eriksson, H.-E. and Penker, M. (1998). UML Toolkit. John Wiley \& Sons, New York.

Fallside, D. C. (2000). XML Schema part 0: Primer (W3C candidate recommendation 24 October 2000). Available via the World Wide Web at http://www.w3.org/TR/xmlschema-0/.

Gillespie, D. (1977). Exact stochastic simulation of coupled chemical reactions. J. Phys. Chem., 81:23402361.

Gilman, A. (2000). A letter to the signaling community. Alliance for Cellular Signaling, The University of Texas Southwestern Medical Center. Available via the World Wide Web at http://afcs.swmed.edu/ afcs/Letter_to_community.htm.

Harold, E. R. and Means, E. S. (2001). XML in a Nutshell. O'Reilly \& Associates.

Hedley, W. J., Nelson, M. R., Bullivant, D., Cuellar, A., Ge, Y., Grehlinger, M., Jim, K., Lett, S., Nickerson, D., Nielsen, P., and Yu, H. (2001). CellML specification. Available via the World Wide Web at http: //www.cellml.org/public/specification/20010810/cellml_specification.html.

Hopcroft, J. E. and Karp, R. M. (1973). An $n^{5 / 2}$ algorithm for maximum matchings in bipartite graphs. SIAM Journal on Computing, 2(4):225-231. 
Hucka, M., Finney, A., Sauro, H. M., and Bolouri, H. (2001). Systems Biology Markup Language (SBML) Level 1: Structures and facilities for basic model definitions. Available via the World Wide Web at http: //www. sbml.org.

Hucka, M., Finney, A., Sauro, H. M., Bolouri, H., Doyle, J. C., Kitano, H., Arkin, A. P., Bornstein, B. J., Bray, D., Cornish-Bowden, A., Cuellar, A. A., Dronov, S., Gilles, E. D., Ginkel, M., Gor, V., Goryanin, I. I., Hedley, W. J., Hodgman, T. C., Hofmeyr, J.-H., Hunter, P. J., Juty, N. S., Kasberger, J. L., Kremling, A., Kummer, U., Le Novère, N., Loew, L. M., Lucio, D., Mendes, P., Minch, E., Mjolsness, E. D., Nakayama, Y., Nelson, M. R., Nielsen, P. F., Sakurada, T., Schaff, J. C., Shapiro, B. E., Shimizu, T. S., Spence, H. D., Stelling, J., Takahashi, K., Tomita, M., Wagner, J., and Wang, J. (2003). The Systems Biology Markup Language (SBML): A medium for representation and exchange of biochemical network models. Bioinformatics, 19(4):524-531.

Iannella, R. (2001). Representing vCard objects in RDF/XML. Available via the World Wide Web at http://www.w3.org/TR/vcard-rdf.

Jacobs, I. (2004). World wide web consortium process document. Available via the World Wide Web at http://www.w3.org/2004/02/Process-20040205/.

Kokkelink, S. and Schwänzl, R. (2002). Expressing qualified Dublin Core in RDF/XML. Available via the World Wide Web at http://dublincore.org/documents/dcq-rdf-xml/index.shtml.

Lassila, O. and Swick, R. (1999). Resource description framework (RDF) model and syntax specification. Available via the World Wide Web at http://www.w3.org/TR/REC-rdf-syntax/.

Le Novère, N., Finney, A., Hucka, M., Bhalla, U., Campagne, F., Collado-Vides, J., Crampin, E. J., Halstead, M., Klipp, E., Mendes, P., Nielsen, P., Sauro, H., Shapiro, B., Snoep, J. L., Spence, H. D., and Wanner, B. L. (2005). Minimum information requested in the annotation of biochemical models (MIRIAM). Nature Biotechnology, 23:1509-1515.

Oestereich, B. (1999). Developing Software with UML: Object-Oriented Analysis and Design in Practice. Addison-Wesley Publishing Company.

Pemberton, S., Austin, D., Axelsson, J., Celik, T., Dominiak, D., Elenbaas, H., Epperson, B., Ishikawa, M., Matsui, S., McCarron, S., Navarro, Peruvemba, S., Relyea, R., Schnitzenbaumer, S., and Stark, P. (2002). XHTML $^{\mathrm{TM}} 1.0$ the Extensible HyperText Markup Language (second edition): W3C Recommendation 26 January 2000, revised 1 August 2002. Available via the World Wide Web at http://www.w3.org/TR/ $\mathrm{xhtml1/.}$

Popel, A. and Winslow, R. L. (1998). A letter from the directors.... Center for Computational Medicine \& Biology, Johns Hopkins School of Medicine, Johns Hopkins University. Available via the World Wide Web at http://www. bme.jhu.edu/ccmb/ccmbletter.html.

Powell, A. and Johnston, P. (2003). Guidelines for implementing Dublin Core in XML. Available via the World Wide Web at http://dublincore.org/documents/dc-xml-guidelines/index.shtml.

Reder, C. (1988). Metabolic Control Theory: a structural approach. Journal of Theoretical Biology, 135:175201.

Sauro, H. M. and Ingalls, B. (2003). Conservation analysis in biochemical networks: Computational issues for software writers. Available via the World Wide Web at http://www.math. uwaterloo.ca/ `bingalls/ Pubs/conservation.pdf.

Smaglik, P. (2000). For my next trick.... Nature, 407:828-829.

Thompson, H. S., Beech, D., Maloney, M., and Mendelsohn, N. (2000). XML Schema part 1: Structures (W3C candidate recommendation 24 October 2000). Available via the World Wide Web at http://www. w3. org/TR/xmlschema-1/. 
Unicode Consortium (1996). The Unicode Standard, Version 2.0. Addison-Wesley Developers Press, Reading, Massachusetts.

W3C (2000a). Naming and addressing: URIs, URLs, .... Available via the World Wide Web at http: //www.w3.org/Addressing/.

W3C (2000b). W3C's math home page. Available via the World Wide Web at http://www.w3.org/Math/.

W3C (2004a). Rdf/xml syntax specification (revised). Available via the World Wide Web at http://www. w3.org/TR/rdf-syntax-grammar/.

W3C (2004b). Resource description framework (RDF). Available via the World Wide Web at http://www . w3. org/RDF/.

Wilkinson, D. J. (2006). Stochastic Modelling for Systems Biology. Chapman \& Hall/CRC.

Wolf, M. and Wicksteed, C. (1998). Date and time formats. Available via the World Wide Web at http: //www.w3.org/TR/NOTE-datetime.

Zwillinger, D., editor (1996). Standard Mathematical Tables and Formulae. CRC Press LLC, 30th edition. 